\title{
Intra-genomic variation in symbiotic dinoflagellates: recent divergence or natural hybridization?
}

\author{
Shaun Peter Wilkinson
}

A thesis submitted to the Victoria University of Wellington in fulfilment of the requirements for the degree of Doctor of Philosophy

Victoria University of Wellington

2015 



\section{Abstract}

The perpetuity of coral reefs will ultimately depend on the ability of corals to adapt to changing conditions. Inter-specific hybridization can provide the raw genetic material necessary for adaptation, and stimulate macro-evolutionary leaps during periods of environmental upheaval. Though well-documented in corals, hybridization has yet to be identified in their dinoflagellate symbionts (genus Symbiodinium), despite growing evidence of sexual reproduction in this genus. The integral roles that these symbiotic algae play in coral productivity, reef accretion and 'coral bleaching' emphasize the need to better understand their short-term evolutionary potential. In this thesis, I develop new molecular and statistical methodology, and combine lab- and field-based analysis to explore the potential for hybridization between divergent Symbiodinium taxa.

To screen for putative Symbiodinium hybrids, intra-genomic variation was examined within individual symbionts isolated from the reef-building coral Pocillopora damicornis at Lord Howe Island (Australia). A nested quantitative PCR (qPCR) assay was developed to quantify polymorphic internal transcribed spacer 2 (ITS2) sequences within the genome of each symbiont cell. Three genetically distinct Symbiodinium populations were detected co-existing within the symbiont consortium of $P$. damicornis. Mixed populations of 'pure' Symbiodinium types C100 and C109 coexisted with a population of cells hosting co-dominant C100 and C109 ITS2 repeats. Genetically heterogeneous Symbiodinium cells were more common than homogeneous symbionts in four of the six colonies analysed, with a maximum proportional abundance of $89 \%$.

Morphological, functional and ecological attributes of heterogeneous Symbiodinium cells were characterized to assess their candidacy as putative hybrids. The proportional abundance of genetically heterogeneous symbionts was spatially and temporally conserved within colonies, indicating a lack of competition between Symbiodinium populations. However, this abundance ratio varied considerably between colonies separated by metres to tens of metres, and to a greater extent between sites isolated by hundreds to thousands of metres. The local thermal maximum emerged as a significant predictor of the proportional abundance of genetically heterogeneous Symbiodinium cells, suggesting that the distribution of these 'putative hybrids' is influenced by a reduced affinity for thermal stress. 
Genetically heterogeneous Symbiodinium cells were around 50\% larger (by volume) than homogeneous cells, occupied tissue of the coral host at reduced densities, and showed relatively poor light-harvesting efficiency. Colonies hosting a higher proportion of these symbionts suffered a reduction in overall photosynthetic performance (maximum gross photosynthesis normalised to respiration; $P: R$ ) at the ambient temperature of $25^{\circ} \mathrm{C}$. This disparity was maintained when the temperature was elevated to simulate the maximum experienced within the LHI lagoon $\left(29^{\circ} \mathrm{C}\right)$. Under these stressful conditions, colonies dominated by putative Symbiodinium hybrids were only marginally capable of net oxygen production.

The influence of putative Symbiodinium hybrids on the growth and survival of $P$. damicornis was tested by reciprocally transplanting coral colonies between reef sites featuring distinct temperature regimes. Neither calcification nor mortality was influenced by the proportional abundance of genetically heterogeneous cells in the symbiont consortium. This uncoupling of symbiont performance and host fitness may be explained by stochastic events such as predation and disease, which substantially increase variation in growth and mortality in field experiments. Alternatively, it may represent some unknown benefit associated with hosting hybrid symbionts, belying their relatively poor photosynthetic performance, and explaining the widespread abundance of these heterogeneous Symbiodinium cells on the Lord Howe Island reef.

Our inability to maintain many clade C Symbiodinium types in culture prevents direct observations of hybridization between C100 and C109. Unequivocal evidence of this phenomenon will therefore likely remain elusive until high-resolution, single-copy nuclear markers can be developed, since the incomplete displacement of ancestral polymorphisms can leave a similar genomic signature to that of hybridization. However, this study serves to provide an initial proof-of-principle for hybridization between divergent Symbiodinium taxa. In doing so, it highlights the need to better understand the evolutionary processes underpinning coral- and symbiont-adaptation in a changing climate. 


\section{Contributions and publications}

This thesis is compiled as a series of manuscripts that have been submitted for publication or are in the preparation process. The thesis is the intellectual and analytical work of the author, with contributions as described below. Assoc. Prof. Simon Davy and Dr. Paul Fisher provided supervisory, intellectual and editorial assistance throughout, and Dr. Stefanie Pontasch assisted with field-work at Lord Howe Island.

Chapter 2: This chapter is formatted as an independent manuscript: Wilkinson SP, Fisher PL, Davy SK. Intra-genomic variation in symbiotic dinoflagellates: recent divergence or natural hybridization? This manuscript has been submitted for publication.

Chapter 3: This chapter is formatted as an independent manuscript: Wilkinson SP, Pontasch S, Fisher PL, Davy SK. The spatiotemporal distribution of putative Symbiodinium hybrids at Lord Howe Island, Australia.

Chapter 4: This chapter is formatted as an independent manuscript: Wilkinson SP, Pontasch S, Fisher PL, Davy SK. Poor functional performance of putative Symbiodinium hybrids and their coral hosts at a high-latitude, marginal coral reef.

Chapter 5: This chapter is formatted as an independent manuscript: Wilkinson SP, Pontasch S, Fisher PL, Davy SK. Reduced functional performance in putative Symbiodinium hybrids does not impair coral growth and survival in their natural habitat.

Financial support was provided by a VUW Vice Chancellor's Strategic PhD Scholarship, a J. L. Stewart Scholarship, a VUW PhD Submission Scholarship and a Faculty Strategic Research Grant, awarded to the author. Additional assistance for field-work costs was provided by a Royal Society Marsden Fund Grant awarded to Assoc. Prof. Simon Davy and Dr. Paul Fisher (contract number VUW0902). Corals were collected under New South Wales Department of Primary Industries permit number P10/0042-1.1. The work was made possible with the help of the Lord Howe Island Marine Parks Authority. 


\section{Acknowledgements}

I am very grateful for the sources of funding that made this project possible. In particular, I wish to extend my gratitude to Victoria University of Wellington for deeming me a worthy recipient of the VUW Vice Chancellor's PhD Scholarship.

This thesis represents a large amount of work, much of which came from the efforts of other people. I would initially like to thank Simon Davy for being the consummate supervisor. Simon seemed to have an unwavering belief in me, and remained interested and helpful even when the project drifted far from his academic comfort zone, battling through my stats jargon and always providing valuable feedback. This work would also not have been possible without the help of my co-supervisor Paul Fisher, who willingly passed on his skills and knowledge, and whose original idea it was to attempt genetic quantification on a single symbiont cell.

Carrying out field-work in the pristine Lord Howe Island Marine Park was an extraordinary experience, and I thank all the people who made it possible. I would especially like to thank my research buddy Stefanie Pontasch; our mission was never going to be an easy one, and with resourcefulness, determination, and copious amounts of coffee, we got there! I also wish to thank the people at the Lord Howe Island Marine Parks Authority, particularly Ian Kerr, Sallyann Gudge, Jimmy Maher and Tas Douglass. You guys were so welcoming, helpful, generous, understanding, and actively engaged in our research. Thanks also to Tim Solomon and Rebecca Moran for hosting me on the island, and to the many other locals who helped turn our ambitious plans into a reality. I'd especially like to thank Ian Fitzgerald for volunteering a huge amount of time and effort, and Brian Busteed at Howea Divers for making our diving experience unforgettable. Thanks also to the many academics we met on the island and provided much needed guidance to a couple of early-career researchers. In particular, thanks to Professor Peter Harrison, whose advice I found invaluable.

It seems that all academics have an unmanageable amount of work, and I am very appreciative of those who sacrificed their time to improve the scientific quality of this thesis. I am deeply grateful for the help and advice I received from Shirley Pledger, Nicole Phillips, Xavier Pochon, Michael Stat, Scott Santos, and Joe Zuccarello. Thanks also to Madeleine van Oppen for sharing her unpublished work, and to Ruth Gates for showing an interest in my research and keeping me in high spirits during the write-up. I'd also like to take this 
opportunity to thank the anonymous reviewers who provided detailed and constructive feedback on my earlier manuscript drafts, ultimately making this thesis a much better product. A big thanks to Luke Thomas for inviting me along on his research expedition to the Houtman Abrolhos, and teaching me his own unique brand of resourcefulness. Thanks to Emma Gibbin and Katie Hillyer for compensating for my not-so-good admin skills, and to the rest of the Davy Lab group for providing a stimulating research environment. Cheers to my office mates Luke, Karl and Gagan for making my time at Vic an enjoyable one. Thanks also to everyone that brought my pies and helped me get to the symbiosis conference in Poland!

There are many people who gave me a great deal of personal support along the way. Thanks to my family, Mum, Dad, Naomi and family, Kath, Claire, Jules and my Nana Dawn. I'd especially like to thank my parents Pete and Jen for fostering my interest in science from an early age. Over the past few years I have received an enormous amount of support from the Bourke family, John, Trish, Anna, Mike, Lou, Carel, Frank and Anna. A special thank you to Trish for all the wonderful meals, and to John for convincing me that I should go to university. Without that piece of advice I would be much less stimulated mentally, though probably a lot better off financially. Thanks to Franky for being a good mate, and understanding why I've had to neglect the extreme catamaraning and spearfishing over the past few months. Finally, thanks to my patient and understanding soon-to-be wife Alice, for providing a little extra encouragement to get this thesis into the hand-in box, and for making life great. 


\section{List of abbreviations}

$\alpha$

ABH

AFLP

AGF

AIC

AICc

AIMS

ANCOVA

ANOVA

BLAST

BSA

bp

$\mathbf{C}_{\mathrm{C} 100}$

$\mathrm{CO}_{2}$

$\mathrm{C}_{\mathrm{t}}$

$\mathbf{C}_{\text {Total }}$

CDS

Chl

df

DGGE

DMSO

DNA

DNAB

EDTA

ENSO
Light-harvesting efficiency

Adaptive bleaching hypothesis

Amplified fragment length polymorphism

Assisted gene-flow

Akaike information criterion

Corrected Akaike information criterion

Australian Institute of Marine Science

Analysis of covariance

Analysis of variance

Basic local alignment search tool

Bovine serum albumin

Base pair

The total number of Symbiodinium clade C ITS2 sequence copies of type C100

Carbon dioxide

Cycling threshold value

The total number of Symbiodinium clade C ITS2 sequence copies

Coding sequence

Chlorophyll

Degrees of freedom

Denaturing gradient gel electrophoresis

Dimethyl sulfoxide

Deoxyribonucleic acid

DNA buffer

Ethylenediaminetetraacetic acid

El Niño Southern Oscillation 
FISH

FSW

GBR

GPS

$\mathrm{H}_{2} \mathrm{O}$

$\mathrm{HCl}$

HKY

HRF

ICEAP

$I_{C}$

$I_{K}$

IPTG

ITS2

L-BFGS-B

LHI

LHIMPA

LSU

MANCOVA

MANOVA

MINQUE

MLG

MS

$\mathrm{NaCl}$

NDP

NGS

$\mathbf{O}_{2}$
Fluorescence in situ hybridization

Filtered seawater

Great Barrier Reef

Global positioning system

Water

Hydrochloric acid

Hasegawa Kishino Yano model

Host release factor

Incomplete concerted evolution of ancestral polymorphisms

Compensation irradiance

$50 \%$ saturating irradiance

Isopropyl $\beta$-D-1-thiogalactopyranoside

Internal transcribed spacer 2

Limited memory Broyden-Fletcher-Goldfarb-Shanno algorithm with bound constraints

Lord Howe Island

Lord Howe Island Marine Park Authority

Large subunit of ribosome-coding DNA

Multivariate analysis of covariance

Multivariate analysis of variance

Minimum norm quadratic unbiased estimation

Multi-locus genotype

Mean squared error

Sodium chloride

Non-diagnostic polymorphism

Next generation sequencing

Molecular oxygen 
$\boldsymbol{P}: \boldsymbol{R} \quad$ Ratio of maximum gross photosynthesis to respiration

PAGE Poly-acrylamide gel electrophoresis

PAM Pulse amplitude modulation

PAR Photosynthetically active radiation

PCR Polymerase chain reaction

$\boldsymbol{P}_{\text {GROSS }} \quad$ Maximum gross photosynthetic rate

PI Photosynthesis-irradiance

$\boldsymbol{P}_{N E T} \quad$ Maximum net photosynthetic rate

$\boldsymbol{p s} \boldsymbol{b} \boldsymbol{A}^{n \boldsymbol{n} r} \quad$ Non-coding region of the plastid psbA minicircle

qPCR Quantitative PCR, also known as real-time PCR or quantitative realtime PCR

$\boldsymbol{R} \quad$ Respiration rate

RAPD Random amplified polymorphic DNA

rDNA Ribosome-coding DNA

rmANOVA Repeated measures analysis of variance

RNA Ribonucleic acid

$\mathbf{R}_{\mathbf{n}} \quad$ Fluorescence normalized to specific passive dye signal (qPCR)

ROS Reactive oxygen species

Rubisco Ribulose-1,5-bisphosphate carboxylase/oxygenase

SCPCR Single-cell PCR

SCUBA Self-contained underwater breathing apparatus

SE $\quad$ Standard error

$S_{\mathrm{H}}: S_{\mathrm{T}} \quad$ Abbreviated form of Symbiodinium ${ }_{\text {HETEROGENEOUS: }}$

Symbiodinium $_{\text {TOTAL, the proportional abundance of genetically }}$

heterogeneous Symbiodinium cells in the symbiont consortium.

SS

Sum of squared errors

SSU

Small subunit of ribosome-coding DNA

TAE

Tris-acetate-EDTA 
TE

$\mathbf{T}_{\mathbf{m}}$

UK

UV

VUW

WGA

$w_{i}$

XGAL
Tris-EDTA

Melting temperature (qPCR product)

United Kingdom

Ultraviolet

Victoria University of Wellington

Whole genome amplification

Akaike weight

5-bromo-4-chloro-3-indolyl- $\beta$-D-galactopyranoside 


\section{Glossary}

Acclimation Changes that occur within an organism in response to experimentallyinduced changes in its environment

Acclimatization Changes that occur within an organism in response to changes in its natural environment

Adaptation

A heritable modification that makes an organism more fit for existence under the conditions of its environment

Admixture

A population consisting of two or more divergent lineages that undergo interbreeding/hybridization

Allopolyploid An individual carrying two or more sets of chromosomes originating from different lineages

Backcrossing The crossing of fertile hybrids with individuals from one or both progenitor populations

Cross-hybridization An artifactual fluorescence signal arising from a lack of sequence specificity in a fluorogenic probe (qPCR)

Diagnostic locus: A locus that is fixed for alternate alleles in different species

Diploid

An organism/cell carrying twice the haploid number of chromosomes

Epistasis

The interaction between genes at two or more loci resulting in a different phenotype to that expected under independent expression

Excystment

Emergence from a resting cyst stage

Fitness

The genetic contribution of an individual to the subsequent generation

Haploid

An organism/cell carrying a single set of unpaired chromosomes

Haplontic

A type of sexual life-cycle where haploid gametes fuse to form a diploid zygote, which then undergoes meiosis to restore the haploid condition

Heterosis

Superior fitness in early-generation hybrids (relative to their parents) arising from increased heterozygosity. Also known as 'hybrid vigour'

Hermatypic Reef-building (coral)

Heterogeneous Characterized by a co-dominant or near co-dominant mixture of genetic sequences

Heterotrophy A feeding mode involving the assimilation of organic carbon 
Holobiont

Homogeneous

Hybridization

Hypnozygote

Introgression

Nested PCR

Ortholog

Paralog

Polyploid

Progenitor

Ribotype

Scleractinia

Transgressive segregation The production of $\mathrm{F}_{2}$, backcross, or later-generation hybrids with more extreme phenotypes than the phenotypic range of the parental populations

The community of organisms constituting the entire coral entity Characterized by a pure or near-pure array of genetic sequences The crossing of individuals from genetically-divergent lineages A resting cyst produced from the union of two haploid gametes The flow of genes between lineages, arising from the progressive backcrossing of $F_{1}$ and later-generation hybrids with progenitors PCR amplification in two or more stages, using a series of primers that bind to progressively inner regions of the target sequence

A sequence variant arising from the divergence of separate lineages A sequence variant arising within a lineage as a result of gene or genome duplication

An organism/cell carrying more than twice the haploid number of chromosomes

One of the original ancestors of a hybrid lineage

A particular rDNA nucleotide sequence

The order of corals that includes reef-building species (Class: Anthozoa, Subclass: Hexacorallia) 


\section{Table of contents}

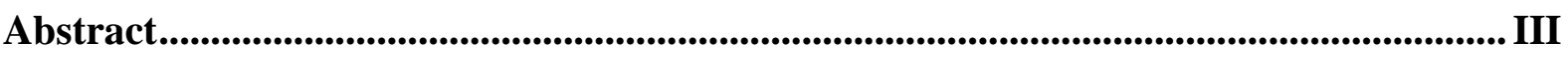

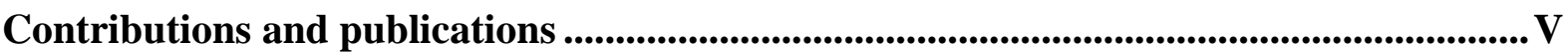

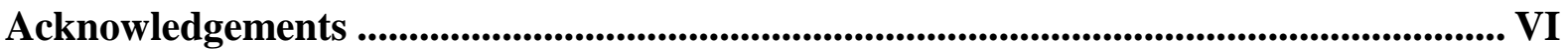

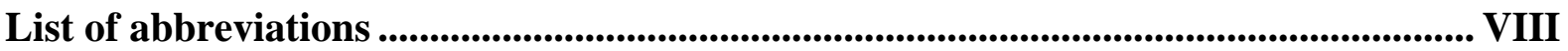

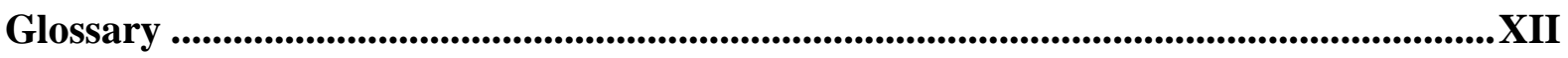

Table of contents ..................................................................................................................... XIV

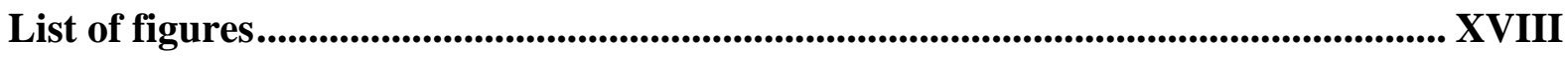

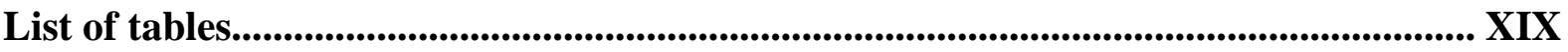

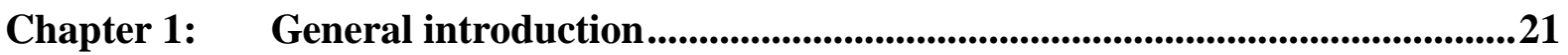

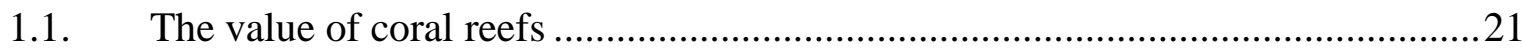

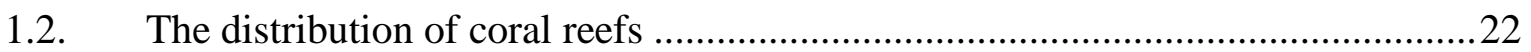

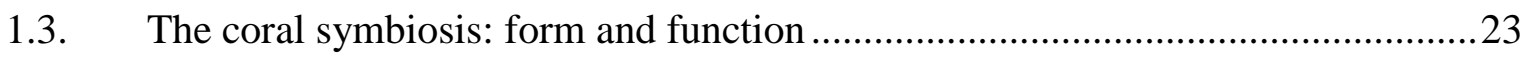

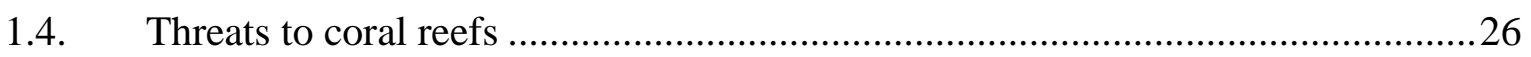

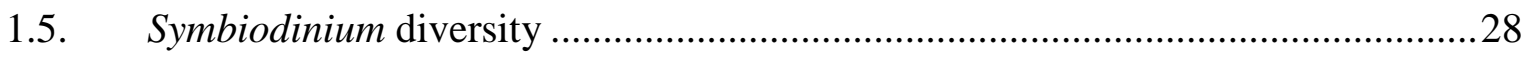

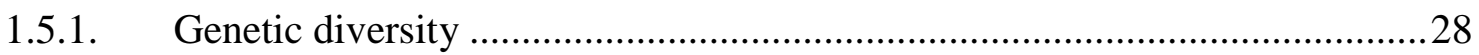

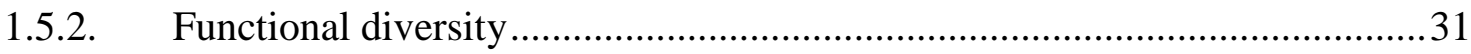

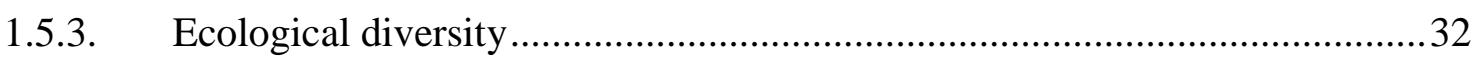

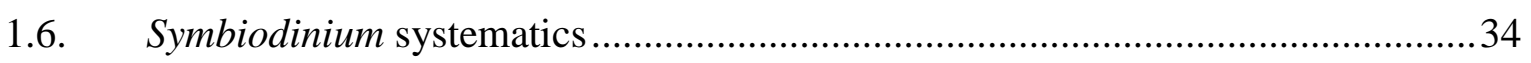

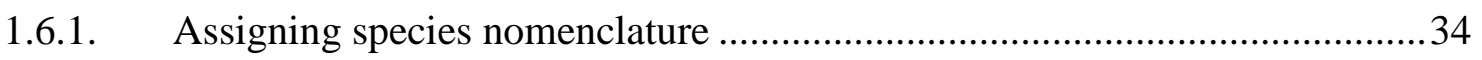

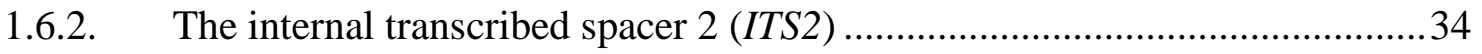

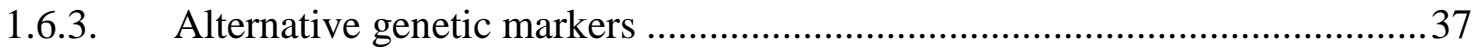

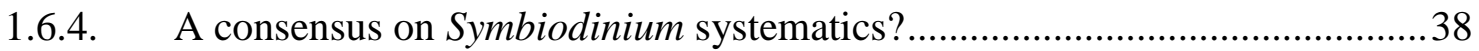

1.7. Coral-symbiont adaptation and acclimatization .............................................. 40

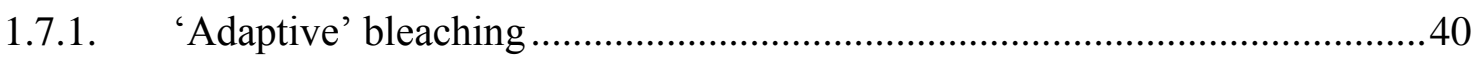

1.7.2. Mixed symbiont populations and symbiont shuffling ................................ 41

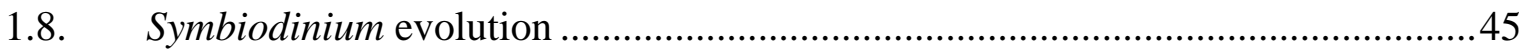

1.8.1. Asexual reproduction in Symbiodinium ........................................................ 45

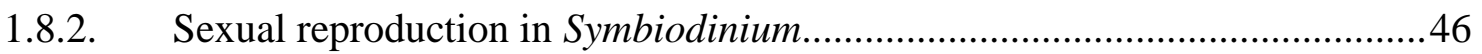

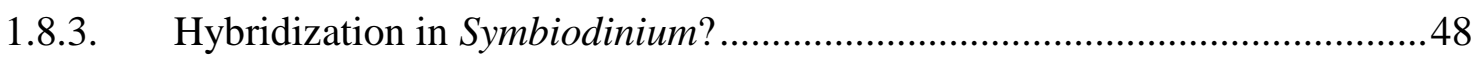

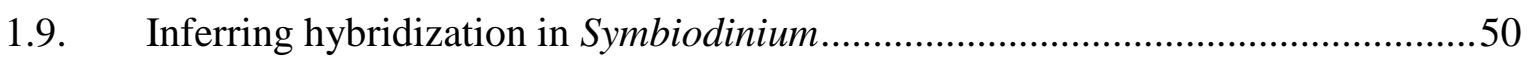

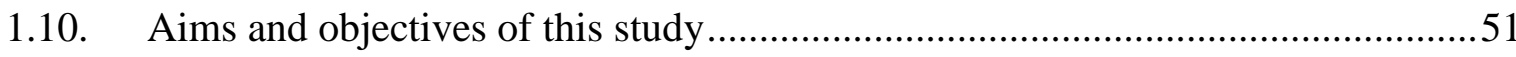


Chapter 2: Measuring intra-genomic variation in Symbiodinium

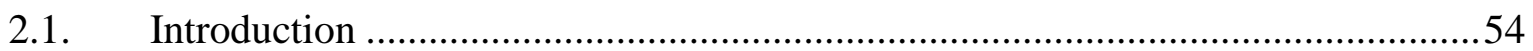

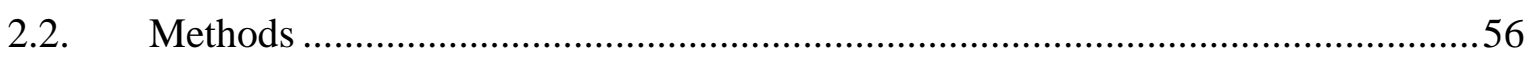

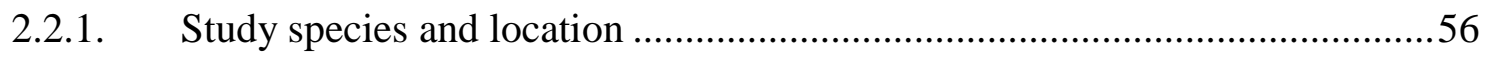

2.2.2. Sample collection and DNA isolation .....................................................57

2.2.3. End-point PCR, DGGE and DNA sequencing ........................................58

2.2.4. qPCR analysis of Symbiodinium ITS2 ratios.............................................59

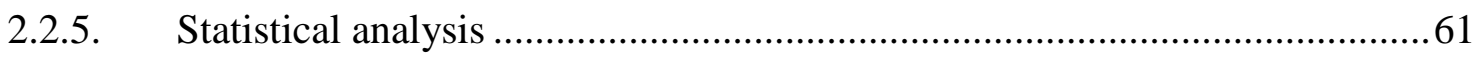

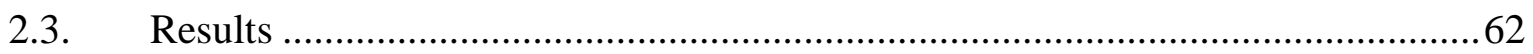

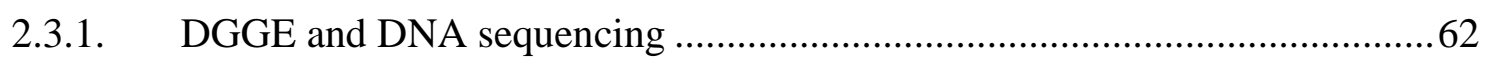

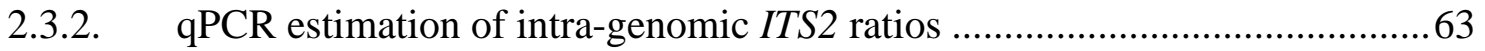

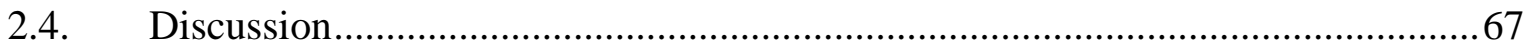

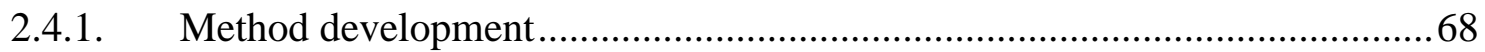

2.4.2. Hybridization or incomplete concerted evolution of ancestral

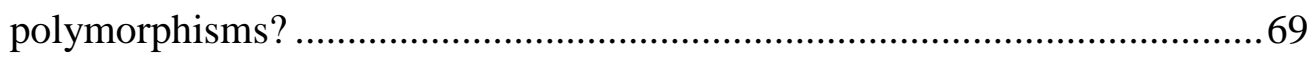

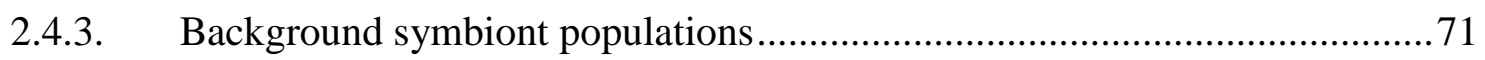

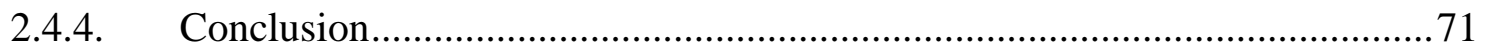

Chapter 3: $\quad$ The spatiotemporal distribution of putative Symbiodinium hybrids........73

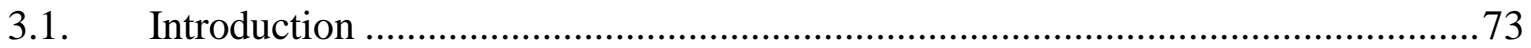

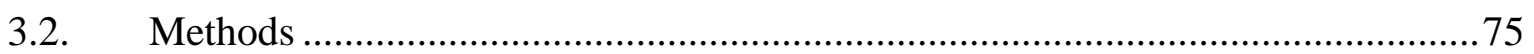

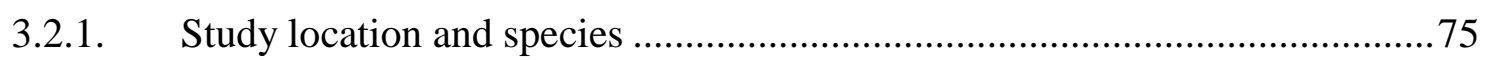

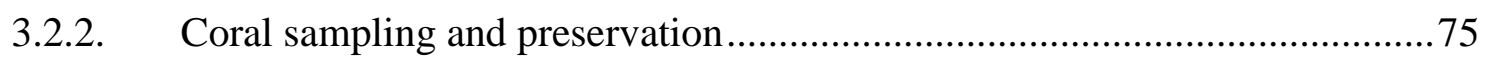

3.2.3. Extraction of bulk-cell Symbiodinium DNA …............................................ 78

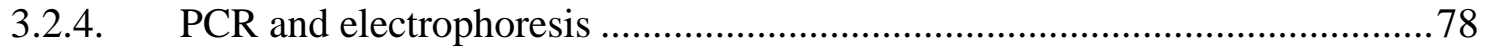

3.2.5. Quantification of genetically heterogeneous Symbiodinium cells.................. 79

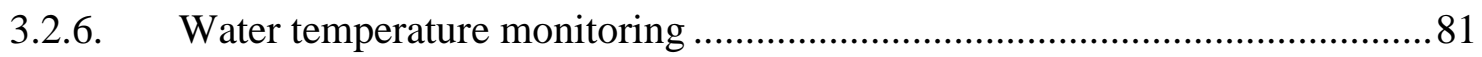

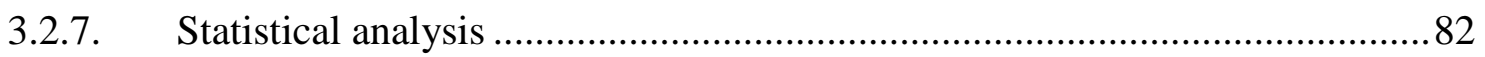

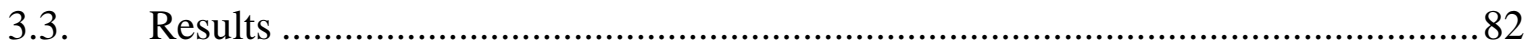

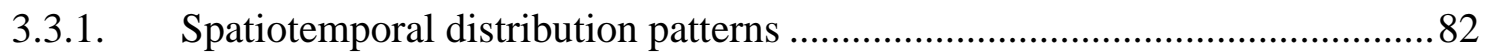

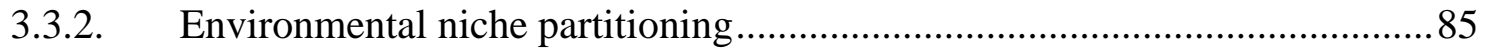

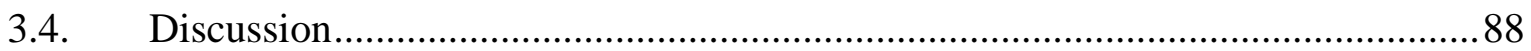

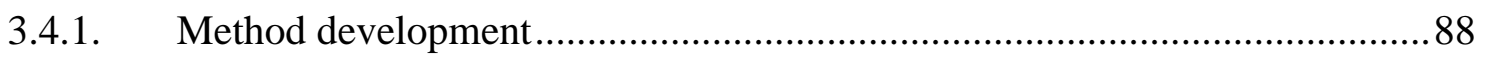

3.4.2. The spatiotemporal distribution of putative Symbiodinium hybrids ..............89 
3.4.3. Niche partitioning of putative Symbiodinium hybrids and progenitors .........91

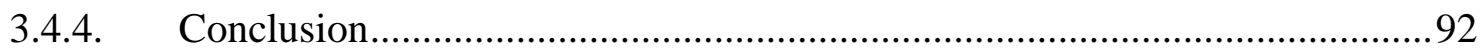

Chapter 4: The form and function of putative Symbiodinium hybrids.........................93

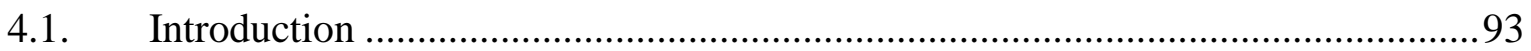

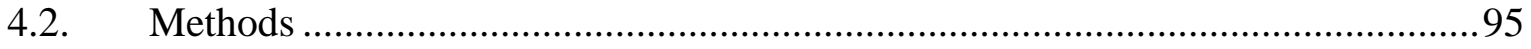

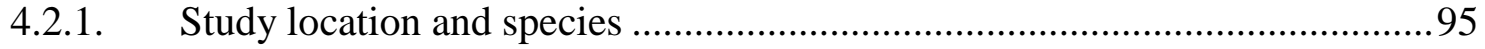

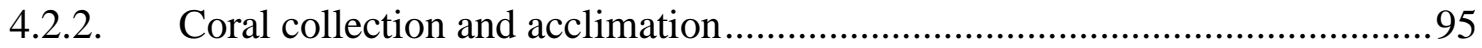

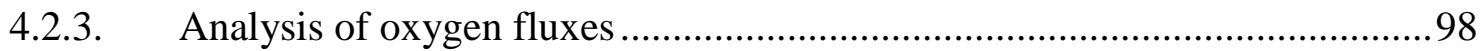

4.2.4. Assessment of morphology and physiology............................................. 99

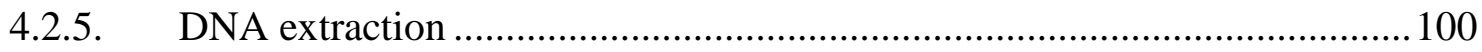

4.2.6. PCR and denaturing gradient gel electrophoresis ................................... 101

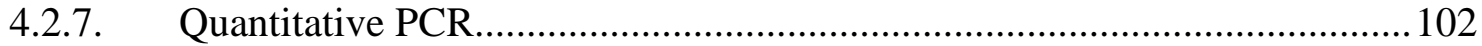

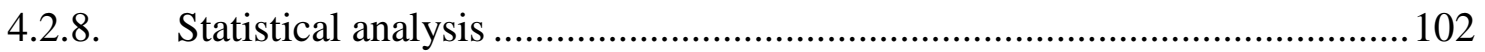

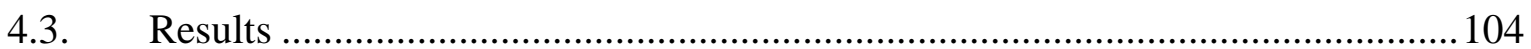

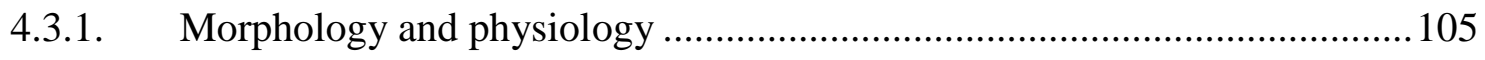

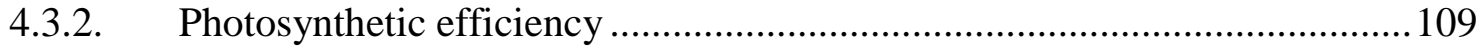

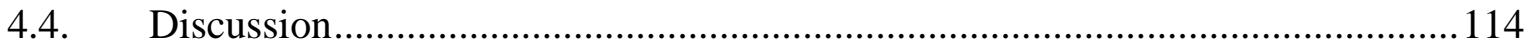

4.4.1. Physical attributes of putative Symbiodinium hybrids ................................ 114

4.4.2. Relative photosynthetic performance of putative Symbiodinium hybrids.... 116

4.4.3. Implications of reduced performance in putative Symbiodinium hybrids....117

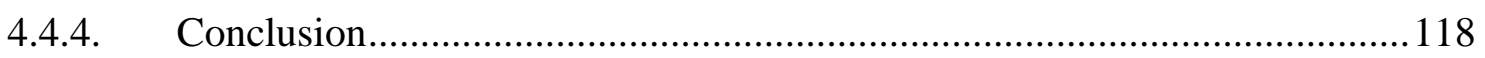

Chapter 5: $\quad$ Symbiont hybridization and coral fitness ......................................................119

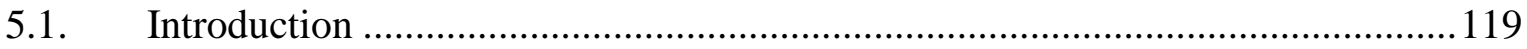

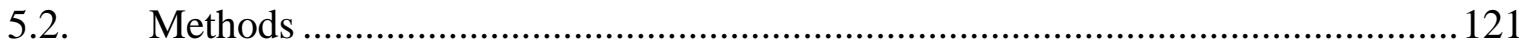

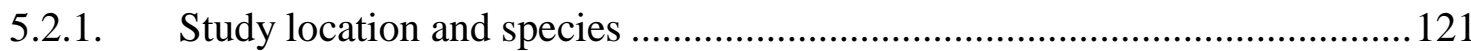

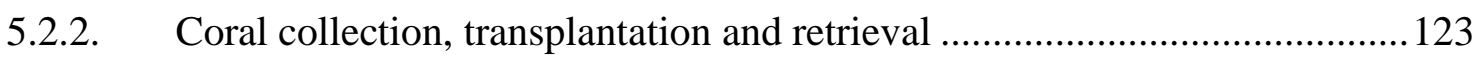

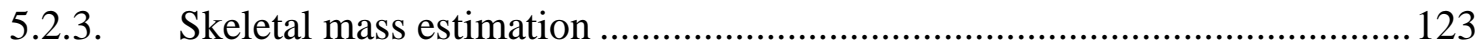

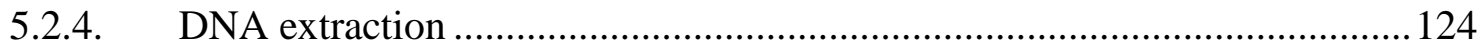

5.2.5. PCR and denaturing gradient gel electrophoresis ................................... 125

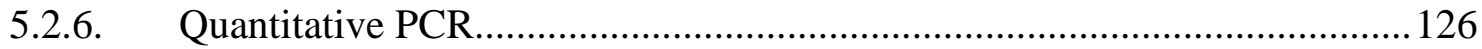

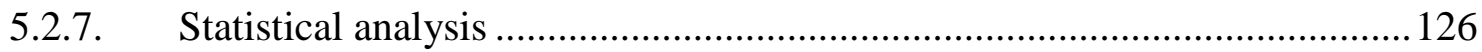

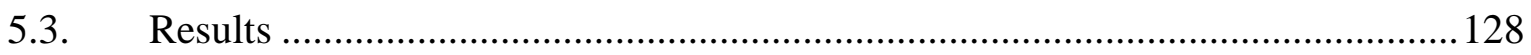

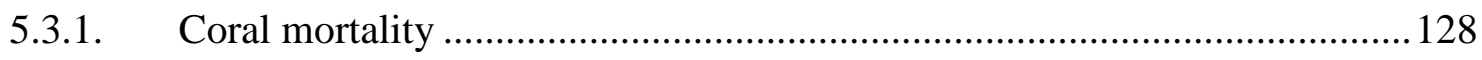




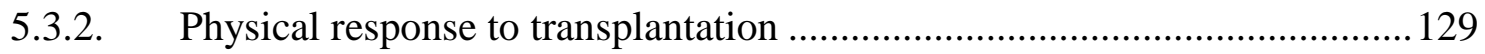

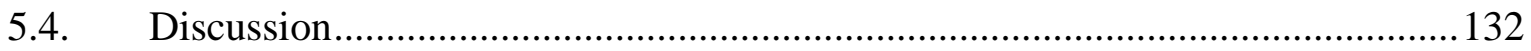

5.4.1. The influence of putative Symbiodinium hybrids on coral fitness ............... 132

5.4.2. Changes in symbiont ratios following transplantation ............................... 133

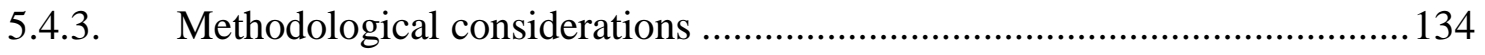

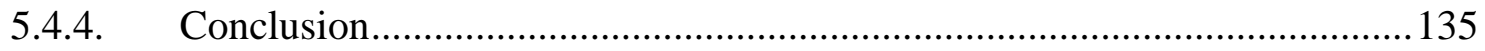

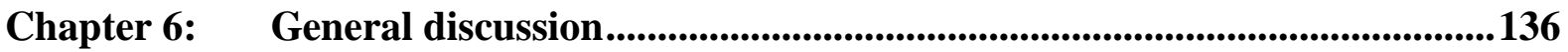

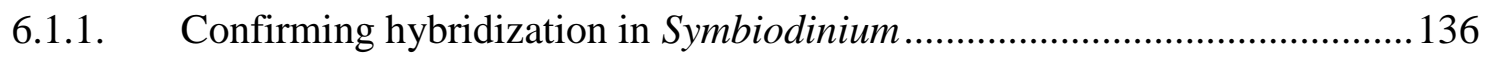

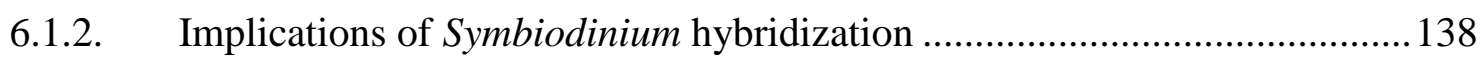

6.1.2.1. When, where and how does hybridization occur? ................................. 139

6.1.2.2. How does symbiont hybridization affect coral fitness?........................... 144

6.1.2.3. What are the evolutionary implications of symbiont hybridization? ....... 146

6.1.2.4. Could hybridization be applied to improve reef resilience? ..................... 147

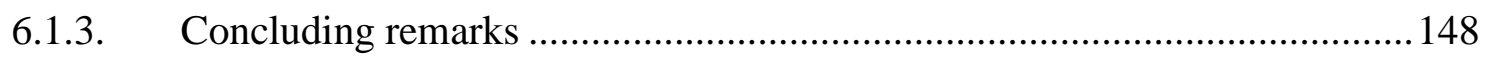

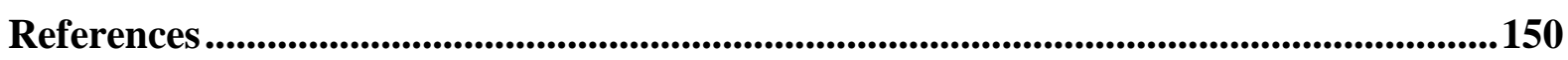

Appendix A: Nucleotide sequences and alignments ...........................................................184

Appendix B: Supplementary data tables and figures ..................................................187 


\section{List of figures}

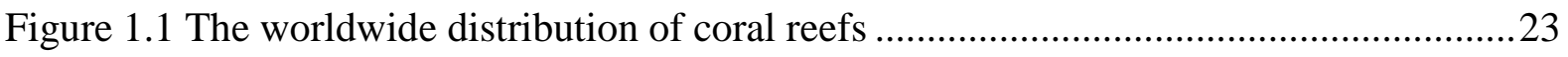

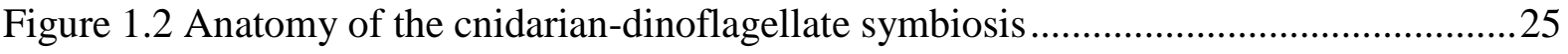

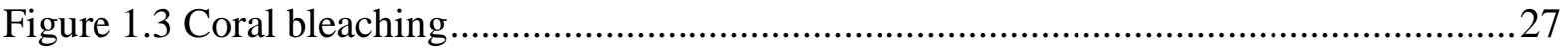

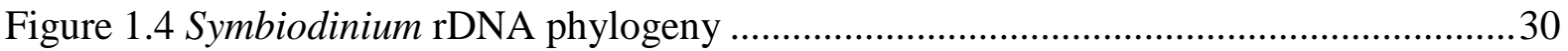

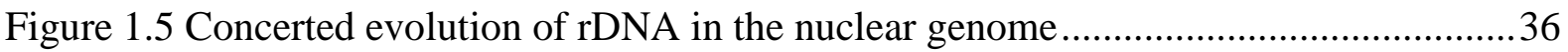

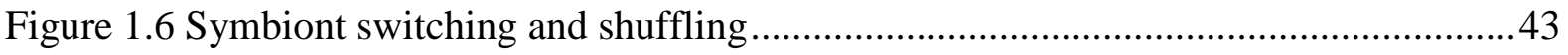

Figure 1.7 The putative haplontic life-cycle of Symbiodinium .............................................4

Figure 2.1 Conflicting origins of intra-genomic variation in Symbiodinium ..........................56

Figure 2.2 Sequence variation among Pocilloporid-associated Symbiodinium at LHI............63

Figure 2.3 Variation in ITS2 copy numbers within Symbiodinium cells ...............................64

Figure 2.4 Frequency distributions of intra-genomic ITS2 ratios in coral colonies................67

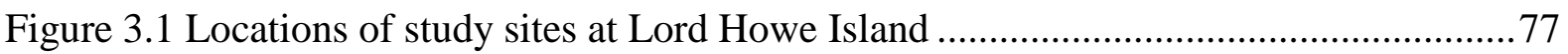

Figure 3.2 Estimating the proportional abundance of putative Symbiodinium hybrids ...........80

Figure 3.3 Thermal characteristics of eight Lord Howe Island reef sites ............................... 86

Figure 3.4 Niche partitioning of putative Symbiodinium hybrids ........................................ 88

Figure 4.1 Thermal acclimation of coral fragments prior to oxygen flux analysis.................97

Figure 4.2 Experimental setup for measuring coral oxygen fluxes .....................................99

Figure 4.3 Properties of the photosynthesis-irradiance (PI) curve ..................................... 104

Figure 4.4 Within-colony variation in symbiont abundance ratios...................................... 105

Figure 4.5 Host protein biomass, symbiont density and cell-size...................................... 106

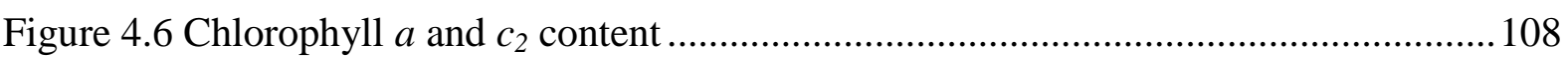

Figure 4.7 Respiratory and photosynthetic oxygen fluxes............................................... 112

Figure 4.8 Photosynthesis-irradiance model for P. damicornis ......................................... 112

Figure 5.1 Location and method of coral transplantation ................................................ 123

Figure 5.2 Effect of putative Symbiodinium hybrids on coral mortality...............................129

Figure 5.3 Physical responses of $P$. damicornis colonies following transplantation ............130

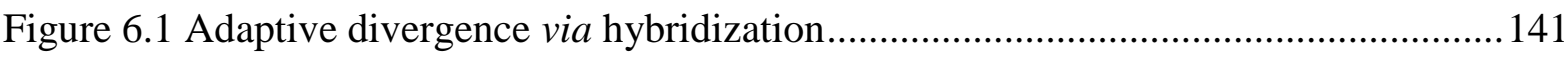

Figure B.1 Single-cell qPCR assay validation (chapter 2) .............................................. 190

Figure B.2 Site-correlation to estimate missing temperature data (chapter 3) ......................205 


\section{List of tables}

Table 1.1 List of formally described Symbiodinium species ............................................... 39

Table 1.2 List of Symbiodinium species lacking formal description (nomen nudum) .............39

Table 2.1 Nested ANOVA for intra-genomic variation in ITS2 ratios ..................................65

Table 2.2 Summary of optimized beta mixture models .......................................................67

Table 3.1 Nested ANOVA for spatial distribution of putative Symbiodinium hybrids ...........84

Table 3.2 Repeated measures ANOVA for temporal changes in putative hybrid abundance. 84

Table 3.3 Reduced nested ANOVA for temporal changes in putative hybrid abundance ......84

Table 4.1 MANCOVA for multi-parameter physiological response .................................... 109

Table 4.2 Univariate ANCOVA for individual physiological response variables.................113

Table 5.1 MANCOVA for coral growth and temporal changes in the proportional abundance of putative Symbiodinium hybrids

Table 5.2 Univariate ANCOVA for coral growth and temporal changes in the proportional abundance of putative Symbiodinium hybrids

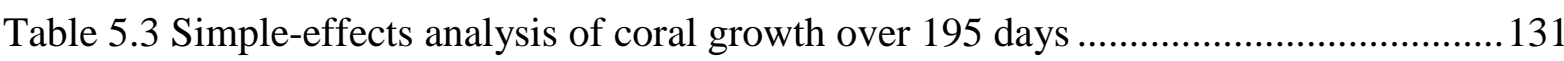

Table 5.4 Simple-effects analysis of coral growth over 374 days ......................................131

Table A.1 Genbank accession numbers for sequences used in rDNA phylogeny ................. 185

Table A.2 Deposition of novel internal transcribed spacer 2 (ITS2) sequence (chapter 2) ... 186

Table B.3 ITS2 sequences used in statistical parsimony network (Figure 2.2) ....................186

Table B.1 Standard curve analysis for nested qPCR (North Bay colonies; chapter 2)..........188

Table B.2 Standard curve analysis for nested qPCR (Ned's Beach colonies; chapter 2) ...... 189

Table B.3 Assay validation for TaqMan nested qPCR (chapter 2)..................................... 190

Table B.4 Mean $C_{t}$ values for individual Symbiodinium cells (colony a; chapter 2)............ 191

Table B.5 Mean $C_{t}$ values for individual Symbiodinium cells (colony b; chapter 2) ............ 192

Table B.6 Mean C $C_{t}$ values for individual Symbiodinium cells (colony c; chapter 2).............193

Table B.7 Mean $C_{t}$ values for individual Symbiodinium cells (colony d; chapter 2) ............ 194

Table B.8 Mean $C_{t}$ values for individual Symbiodinium cells (colony e; chapter 2)............ 195

Table B.9 Mean $C_{t}$ values for individual Symbiodinium cells (colony f; chapter 2) ............. 196

Table B.10 Standard curve parameter estimation for bulk-cell qPCR analysis (chapter 3) .. 197

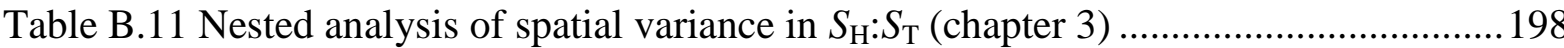

Table B.12 Variation in $S_{\mathrm{H}}: S_{\mathrm{T}}$ along a depth gradient at The Arch (chapter 3) ....................202

Table B.13 Longitudinal analysis of temporal variation in $S_{\mathrm{H}}: S_{\mathrm{T}}($ chapter 3$)$......................203

Table B.14 Climate model parameters for eight LHI study sites (chapter 3) .......................206 
Table B.15 Calculation of $S_{\mathrm{H}}: S_{\mathrm{T}}$ ratios for physiology analysis (chapter 4) ........................207

Table B.16 Physiological diagnostics (chapter 4) ..............................................................2.

Table B.17 Photosynthesis-irradiance measurements (chapter 4) .......................................209

Table B.18 Mortality, growth, and temporal changes in $S_{\mathrm{H}}: S_{\mathrm{T}}$ (chapter 5) ........................210 


\section{Chapter 1: General introduction}

\subsection{The value of coral reefs}

"Anybody who has had the privilege of diving on a coral reef will have seen the natural world at its most glorious, diverse and beautiful". The description given by veteran naturalist and broadcaster Sir David Attenborough accurately depicts the spectacular biodiversity of the reef environment. Coral reefs and their inhabitants generate a valuable global eco-tourism industry, and the economies of many developing countries strongly rely on this source of income (Burke et al. 2011). Yet the economic contribution of the world's coral reefs extends well beyond the tourist dollar. More than $10 \%$ of the world's population lives within $100 \mathrm{~km}$ of a coral reef, and many people directly depend on the reef system for nutrition and coastal stability (Bryant et al. 1998; Burke et al. 2011). Around 10\% of the global fishery catch takes place in reef areas (Smith 1978), and this figure can exceed $25 \%$ in some developing IndoPacific nations (Cesar 1996). Coral reefs also play an important role in climate regulation, and while they comprise only $0.17 \%$ of Earth's ocean surface area (Smith 1978), reefs sequester more atmospheric carbon dioxide per square meter than any other marine ecosystem (Nybakken \& Bertness 2005). Moreover, corals and other reef-associated fauna have been recognized as an important source of bioactive compounds, with the potential to greatly benefit the field of human medicine (Carté 1996).

Placing a monetary value on the goods and services provided by coral reefs is necessary for the purposes of conservation prioritisation (Costanza et al. 1997). Yet this is made difficult by the inter-connectivity of coral reefs with the broader ecological 'seascape' (Ogden 1988). The productivity and complex three-dimensional architecture afforded by coral reefs support an astonishing diversity of flora and fauna in otherwise desolate tropical waters (Odum \& Odum 1955), with estimates of up to 9 million species worldwide (Small et al. 1998). Reefs also promote coastal stability through dissipation of wave energy at reef margins, thus enabling the development of other important tropical ecosystems such as mangrove forests and seagrass beds (Hoegh-Guldberg 1999). Therefore, while current evaluation estimates their ecosystem services at US \$375 billion annually (Costanza et al. 1997), the true value of coral reefs to the human population is certainly much higher. 


\subsection{The distribution of coral reefs}

For net reef accretion to occur, corals require warm temperatures, high levels of photosynthetically active radiation (PAR), and saturation or near-saturation of aragonite, the inorganic substrate necessary for calcification (Barnes \& Chalker 1990). As such, contiguous coral reef structures are predominantly confined within the equatorial band from $30^{\circ} \mathrm{N}$ to 30 ${ }^{\circ} \mathrm{S}$ (Sheppard et al. 2009; Figure 1.1). Maximum diversity occurs within the Indo-Australian archipelago (the 'Coral Triangle'), an area inhabited by some 500 coral species (Briggs 1999; Green \& Mous 2008). Coral diversity generally diminishes with distance from this biodiversity hotspot, with only around 50 species found at the Galapagos Islands (Briggs 1999; Knowlton 2001). At higher latitudes, communities of corals can be found interspersed among macroalgal assemblages; however they do not form contiguous reef structures due to suboptimal abiotic conditions and competition with macroalgae (Veron 1995; Paulay 1997). The world's northernmost coral reefs occur at Bermuda and Okinawa (Japan; $33^{\circ} \mathrm{N}$ ), and the southernmost reef is that of Lord Howe Island (Australia; $31.5^{\circ} \mathrm{S}$ ). Despite being situated at the transition zone between coral- and macroalgal-dominated benthic systems, these 'marginal reefs' can feature high levels of biodiversity. In particular, the isolated reef of Lord Howe Island includes more than 83 scleractinian coral species, many of which are endemic (Veron \& Done 1979; Harriott et al. 1995).

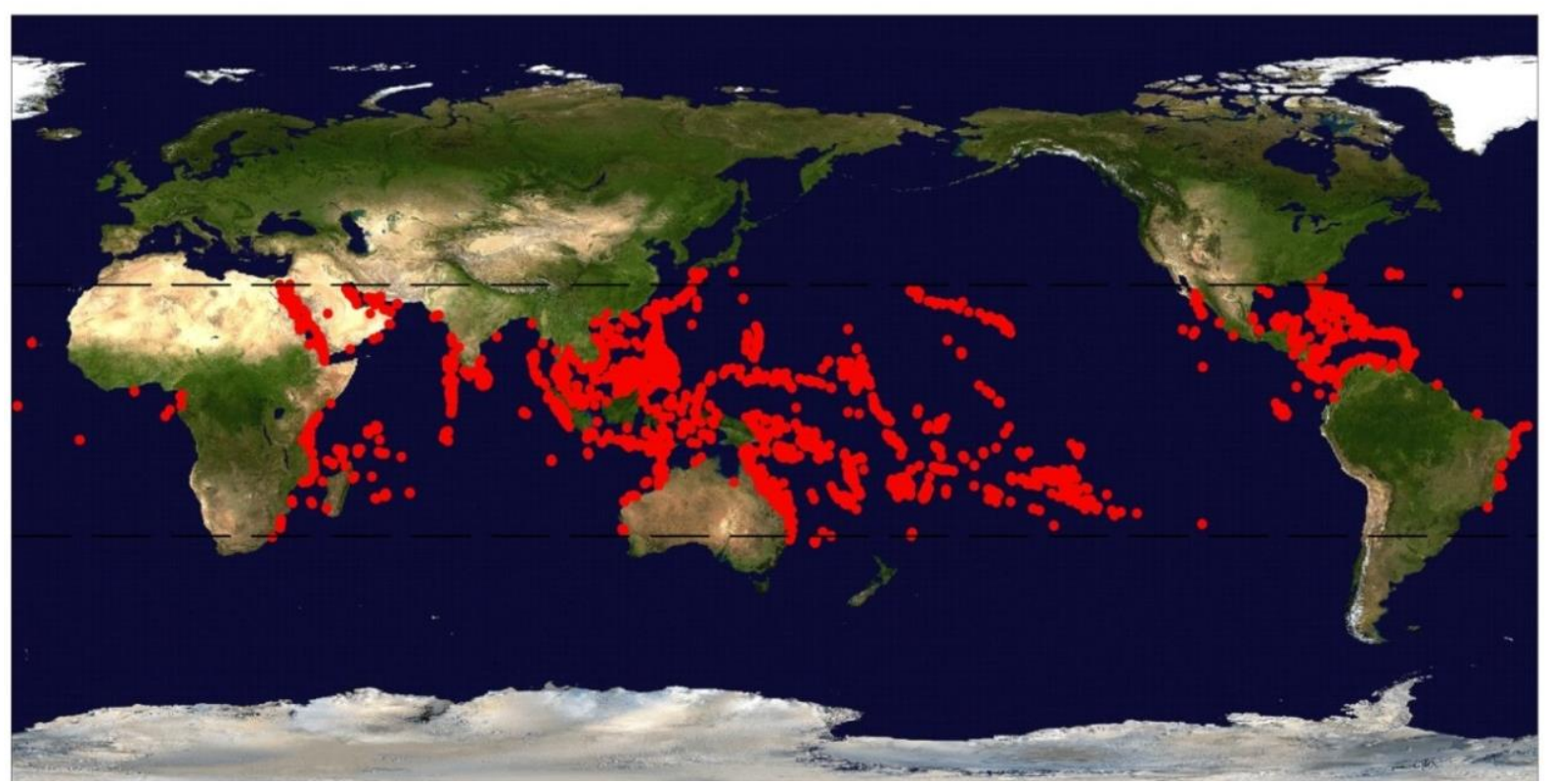


Figure 1.1 (previous page) The worldwide distribution of coral reefs

Dashed lines show the latitudinal band of $30^{\circ} \mathrm{N}$ and $30^{\circ} \mathrm{S}$, within which the majority of coral reefs are concentrated. GPS data and satellite pictures were obtained from ReefBase, with coral reef locations provided by the United Nations Environment Programme World Conservation Monitoring Centre (UNEP-WCMC; downloaded from http://reefgis.reefbase.org/ on 07/07/2014).

\subsection{The coral symbiosis: form and function}

Coral growth and reef formation are fundamentally supported by symbiosis, the persistent intimate coexistence of different species. The entire coral entity (referred to as the 'holobiont') is composed of a diverse community of organisms, including a cnidarian host (Anthozoa: Scleractinia), single-celled dinoflagellate algae of the genus Symbiodinium (also known as 'zooxanthellae'; Freudenthal 1962), and various other micro-eukaryotes, prokaryotes and viruses (Rohwer et al. 2001, 2002). A complex array of inter-specific interactions underlie the function of the coral holobiont, spanning the continuum from mutualism (both partners benefit) to parasitism (one partner benefits to the other's detriment). Yet the ecological prosperity of scleractinian corals primarily stems from the mutualistic symbiosis between the cnidarian host and the dinoflagellate symbiont. The symbiont inhabits the cells of the host's gastrodermis (Trench 1987; Figure 1.2) at densities that can exceed $10^{6}$ cells per $\mathrm{cm}^{2}$ (Drew 1972), releasing photosynthetically-derived organic compounds such as glycerol and/or glucose, fatty acids, organic acids and amino acids (Trench 1971; Whitehead \& Douglas 2003; Davy et al. 2012). The coral host may receive up to $90 \%$ of the total carbon fixed by its symbionts (Steen \& Muscatine 1984), a nutritional contribution that can fulfil its energetic requirements under well-lit conditions (Muscatine 1990). The symbiont also plays an integral role in the nitrogen status of the symbiosis, assimilating inorganic nitrogen from environmental sources (e.g. dissolved nitrate) and waste products of host metabolism (predominantly ammonium), into host-accessible compounds (Wang \& Douglas 1998; Tanaka et al. 2006; Burriesci et al. 2012; Kopp et al. 2013). Moreover, other essential micronutrients such as phosphorus and sulphur are also assimilated into organic compounds by the symbiont and potentially translocated to the host (Cook 1971; Muller-Parker \& D'Elia 1997; Davy et al. 2012). This highly efficient closed-system of nutrient retention and recycling underpins the characteristically high productivity and diversity of coral reefs in nutrient-poor tropical oceans (Goreau \& Goreau 1959; Muscatine \& Porter 1977; Muscatine 1990; Yellowlees et al. 2008). Finally, the photosynthetic activity of the symbiont ultimately 
supports the calcium carbonate deposition of the host, without which reef accretion would not occur (Pearse \& Muscatine 1971; Vandermeulen et al. 1972; Colombo-Pallotta et al. 2010).

The evolution of the cnidarian-dinoflagellate symbiosis has given rise to a wide variety of behavioural and physiological adaptations. Symbionts can be inherited from parent to offspring, via infection of the gamete and/or asexual larvae (vertical transmission), or they can be acquired anew each generation from environmental source populations (horizontal transmission; see Baird et al. 2009 for a review). In some cases, both horizontal and vertical transmission strategies may be employed (Byler et al. 2013). In the case of horizontal symbiont transmission, the establishment and maintenance of a stable endosymbiosis involves a complex system of symbiont recognition, assortment and phagocytosis ('winnowing'; Nyholm \& McFall-Ngai 2004; Dunn \& Weis 2009). The winnowing process involves an array of surface receptor proteins (including glycan-binding lectins), and potentially the exchange of metabolites (reviewed in Davy et al. 2012). Lectin binding is also believed to play a role in the permanent transition of the symbiont from the motile, flagellated free-living phase to the sedentary coccoid form that the symbiont exhibits when in symbiosis (Koike et al. 2004). Once endosymbiosis is established, the symbiont is housed within a membrane complex consisting of a host vacuole (the 'symbiosome membrane') and a series of inner membranes of symbiont origin (Wakefield et al. 2000; Wakefiel \& Kempf 2001). Nutritional exchange across the symbiosome membrane is regulated by a suite of membranebound and cytoplasmic macro-molecules, which may include an enigmatic 'host release factor' (HRF; Gates et al. 1995; Wang \& Douglas 1997; Ritchie et al. 1997). While our understanding of these cellular interactions is in its infancy, emerging 'big-data' fields such as metabolite profiling (metabolomics), high-throughput RNA-transcript and protein sequencing (transcriptomics and proteomics) and whole genome sequencing (genomics) promise to revolutionize our understanding of the functional adaptations underlying the establishment and maintenance of the symbiotic condition (Davy et al. 2012). 


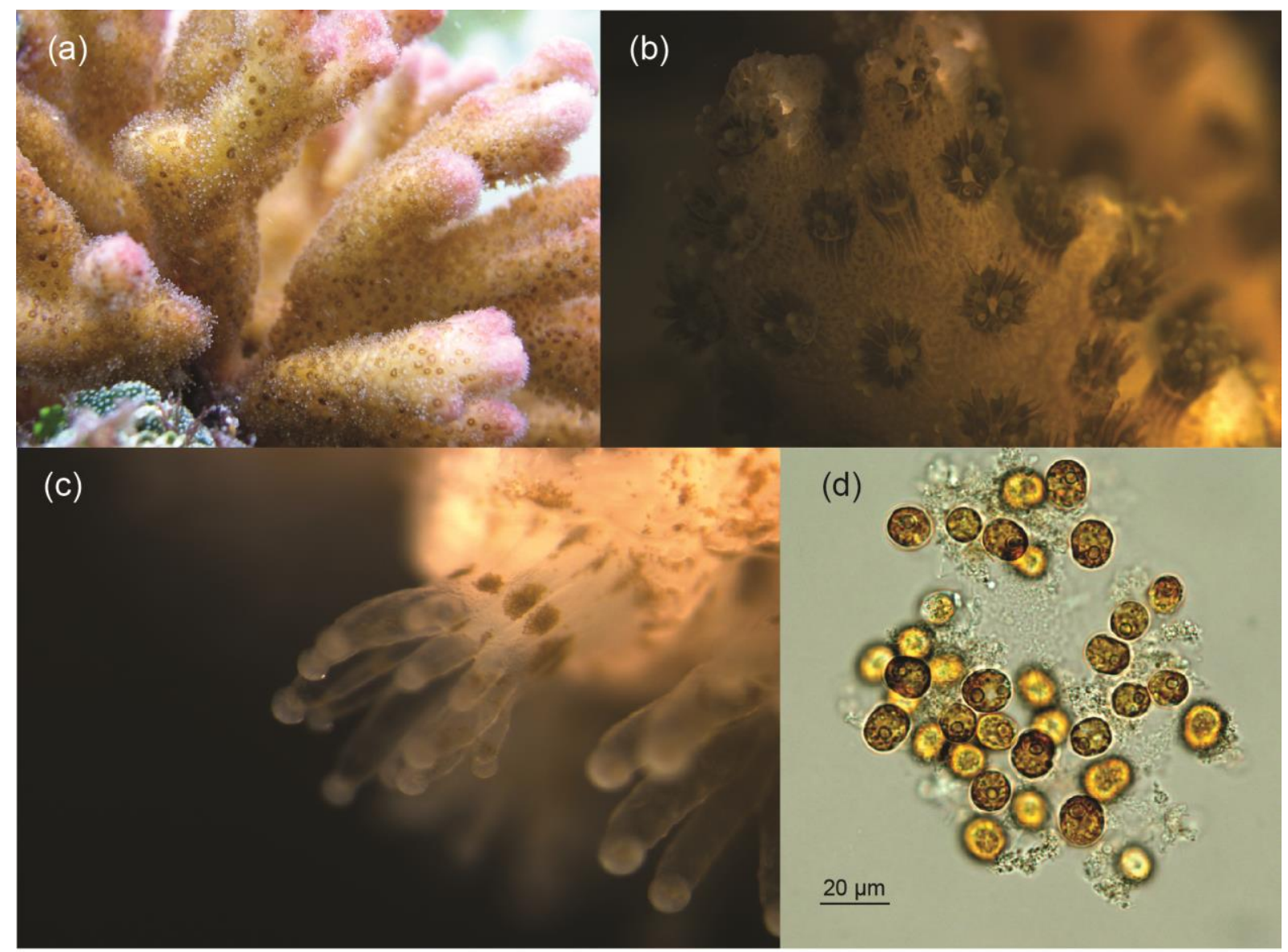

(e)

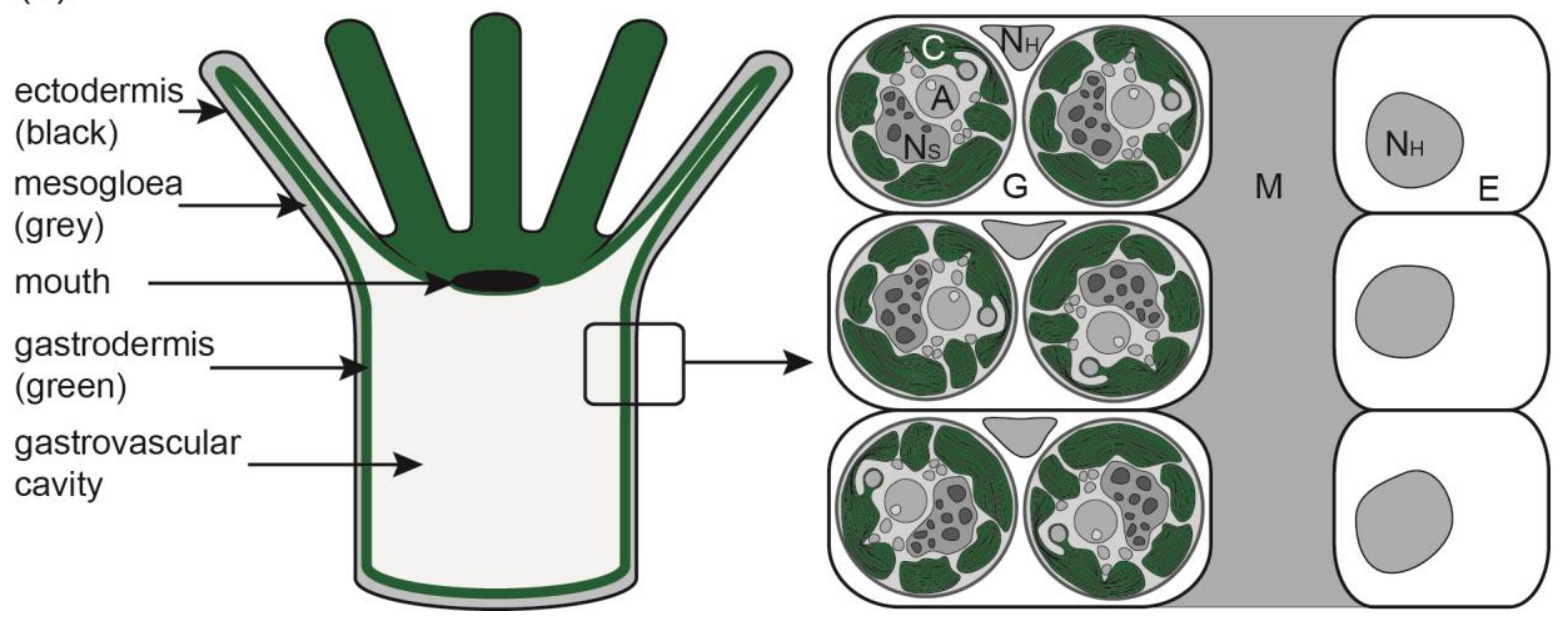

Figure 1.2 Anatomy of the cnidarian-dinoflagellate symbiosis

Scleractinian corals consist of a colony of interconnected clonal polyps with an underlying calcium carbonate skeleton. Images show: (a-c) Pocillopora damicornis with polyps extended, at increasing levels of magnification; (d) coccoid Symbiodinium cells under a light microscope; and (e) schematic cross-section of an individual polyp with a close-up view of the three body layers, the gastrodermis $\mathrm{G}$, the mesogloea M, and the ectodermis E (host nucleus $\mathrm{N}_{\mathrm{H}}$ ). In P. damicornis, a bilayer of coccoid Symbiodinium cells are enclosed in the gastrodermal cells of the host (chloroplast $\mathrm{C}$, accumulation body A, and nucleus $\mathrm{N}_{\mathrm{S}}$; modified from Gates et al. 1992; Allemand et al. 2011). Photo credits: Emma Gibbin (b and c) and Tom Hawkins (d). 


\subsection{Threats to coral reefs}

Corals have evolved in concert with symbiotic dinoflagellates for over 230 million years, forging an obligate association (Coates \& Jackson 1987; Stanley \& Swart 1995; Veron 1995; Glynn 1996). Yet the symbiotic condition renders both partners extremely vulnerable to environmental disturbance. Even marginal abiotic stress can push the 'holobiont' beyond narrowly defined physiological thresholds, leading to symbiotic dysfunction (HoeghGuldberg 1999). Corals are especially vulnerable to elevated temperature (Jokiel \& Coles 1977; Iglesias-Prieto et al. 1992), excess irradiance (Lesser \& Shick 1989; Gleason \& Wellington 1993), and in particular, a synergistic temperature-light interaction (Coles \& Jokiel 1978; Lesser et al. 1990; Fitt \& Warner 1995; Lesser \& Farrell 2004). These factors stimulate the production of reactive oxygen species (ROS) such as hydrogen peroxide, singlet oxygen, and hydroxyl, perhydroxyl, and superoxide anion radicals. ROS are continually produced at low levels in both symbiotic partners, where they are scavenged and neutralized by a suite of antioxidant molecules (reviewed in Lesser 2006). However, ROS can overwhelm the natural antioxidant defences under stressful conditions (Cadenas 1989), disrupting the cellular constituents of both symbiotic partners through protein denaturation, mutagenic DNA damage, and the peroxidation of plasma membranes (particularly those of the mitochondria and chloroplast; Lesser 2006).

A major source of ROS is the thylakoid membrane of the symbiont chloroplast. Here, temperature-induced uncoupling of the photosynthetic electron transport chain can lead to the transfer of electrons to oxygen, forming the highly toxic superoxide anion (the Mehler reaction; Jones et al. 1998). Chronic photoinhibition arises from oxidative damage to the D1protein and plastiquinone binding proteins of photosystem II, and the primary carboxylating enzyme RUBISCO (Renger et al. 1989; Lesser 1996, 1997; Lupínková \& Komenda 2004). The loss of photosynthetic function and increased flux of ROS can induce the coral host to eliminate its symbionts, either by exocytosis (Steen \& Muscatine 1987; Lesser 1996, 1997), detachment of whole gastrodermal cells (Gates et al. 1992), or intracellular degradation (Titlyanov et al. 1996). The loss of symbionts renders the coral tissue transparent, exposing the underlying white coral skeleton in a conspicuous phenomenon known as 'coral bleaching' (Figure 1.3). 


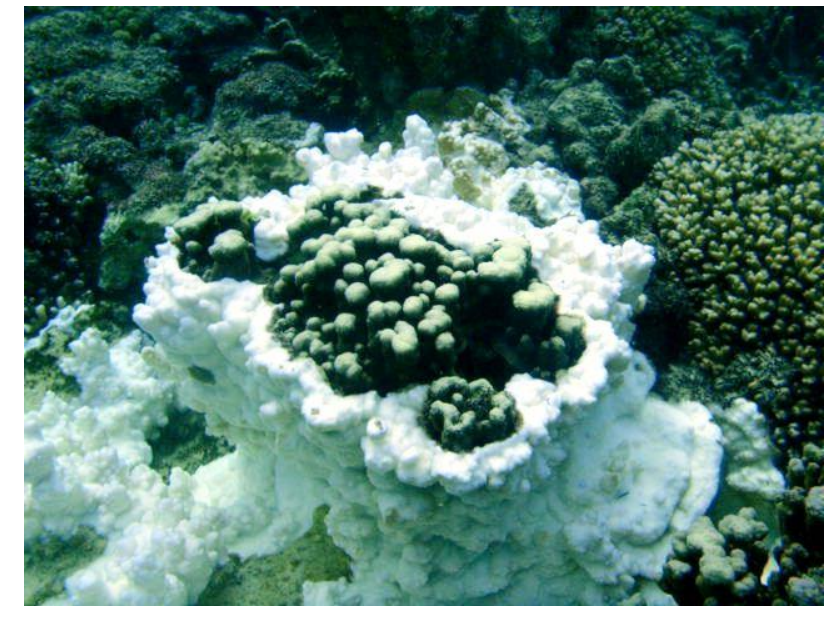

Figure 1.3 Coral bleaching

A partially bleached Porites colony at Lord Howe Island (Australia). The loss of Symbiodinium exposes the underlying coral skeleton, manifesting in a conspicuous white appearance. Bleaching often leads to colony mortality via starvation, unless the coral can quickly regenerate its symbiont population (pending subsistence of environmental stress) or compensate by switching to a heterotrophic feeding mode (e.g. Grottoli $e t$ al. 2006).

Depending on the severity, coral bleaching can result in whole-colony mortality, a process that can occur en masse (Hoegh-Guldberg 1999). The frequency and severity of coral bleaching events has increased in recent decades, primarily due to rises in ocean temperatures associated with anthropogenic carbon dioxide emissions. In a particularly severe case, the 1997-1998 El Niño Southern Oscillation (ENSO) caused a devastating bleaching episode that resulted in significant reef declines in all major geographical areas on Earth (Wilkinson et al. 1999). As such, coral bleaching has been identified as a key threat to the perpetuity of coral reefs in the $21^{\text {st }}$ century (Hughes et al. 2003; Hoegh-Guldberg et al. 2007; Pandolfi et al. 2011).

While bleaching poses a significant threat to the world's corals, a suite of other impacts also endanger these vulnerable organisms. Episodes of coral disease have increased sharply in recent decades as a consequence of elevated temperature and declining habitat quality (Rosenberg \& Ben-Haim 2002; Bruno et al. 2007). Ocean acidification arising from the dissolution of anthropogenic $\mathrm{CO}_{2}$ also poses a significant threat to corals, by inhibiting calcification (Orr et al. 2005), and impeding physiological function (Anthony et al. 2008). As such, a continued reduction below pH 7.7 will likely initiate major ecosystem transitions at the expense of coral reefs (Fabricius et al. 2011; Bell et al. 2013). Moreover, ecosystem disruption arising from overfishing and pollution can lead to outbreaks of corallivores such as the crown-of-thorns starfish (Acanthaster planci; Brodie et al. 2005), and nutrient enrichment from terrestrial runoff and sewage can lower coral performance and fecundity (Tomascik \& Sander 1985, 1987a; b). Through the combined assaults of bleaching, disease, ocean acidification, storm damage, overfishing, destructive fishing, predator outbreaks, pollution, 
coastal development, and other human impacts, the majority of coral species have recently suffered a sharp increase in extinction risk (Carpenter et al. 2008). Concurrently, a prolific research effort has sought to predict the ability of corals to acclimatize and adapt to an environment modified by anthropogenic activity (Hoegh-Guldberg et al. 2007).

\subsection{Symbiodinium diversity}

\subsubsection{Genetic diversity}

Members of the genus Symbiodinium form symbiosis with a diverse array of marine organisms. These include corals, other cnidarians such as zoanthids, octocorals, jellyfish and sea anemones, and several other phyla, including platyhelminths, molluscs, foraminiferans, ciliates and poriferans (Trench 1993; Rowan 1998; Carlos et al. 1999; Pawlowski et al. 2001; Lobban et al. 2002). These symbiotic dinoflagellates were all formerly considered to represent a single pandemic species (Symbiodinium microadriaticum; Freudenthal 1962), a paradigm that was supported by early morphological assessments (Kevin et al. 1969; Taylor 1969, 1974). However, different growth rates of homologous and heterologous algae in situ (Schoenberg \& Trench 1976), and corresponding differences in host performance (Kinzie \& Chee 1979) gave credence to the emerging hypothesis that the genus Symbiodinium harboured a trove of hidden diversity. This was affirmed by early assessments of genetic distance based on isoenzyme polymorphism (Schoenberg \& Trench 1980a), that correlated with subtle differences in size and ultrastructure (Schoenberg \& Trench 1980b). A further correlation was shown between symbiont growth rates within the anemone Aiptasia tagetes (= pallida $)$ and the similarity of isoenzymes to those of the homologous A. tagetes symbiont (Schoenberg \& Trench 1980c). Following these pioneering studies, assessments of host specificity, morphology, physiology and biochemistry were used to describe three new Symbiodinium species (S. goreauii, S. kawagutii and S. pilosum; Trench \& Blank 1987). Yet the apparent absence of sexual reproduction hindered formal genetic analysis (Trench 1987). The increased availability of PCR during the early 1990s facilitated the detection of sequence variation in the highly conserved $n r 18 S$ gene (nuclear ribosomal DNA small-subunit or SSU rDNA Rowan \& Powers 1991a; b, 1992; McNally et al. 1994). The $n r 18 S$ phylogeny was supported by similar topologies obtained from the more rapidly-evolving $n r 28 S$ rDNA region (Wilcox 1998; Pochon et al. 2001), and a hyper-variable region within domain V of the plastid-encoded cp23S gene (Santos et al. 2002). These congruent phylogenetic patterns 
enabled the organization of the Symbiodinium complex into well-supported clades. The recent discovery of a novel Symbiodinium lineage in symbiosis with benthic Foraminifera in Hawaii extends the current phylogeny to nine major taxonomic clades, designated A-I (Pochon \& Gates 2010; Figure 1.4). This delineation is supported by genetic distances that approximate order- or even class-level divergence in free-living dinoflagellates and other algae (Blank \& Huss 1989; Rowan \& Powers 1992), thus effectively invalidating the single-genus classification (Pochon \& Gates 2010). However, a general consensus on Symbiodinium systematics has yet to be reached, primarily due to the existence of substantial genetic diversity within many of the major taxonomic clades.

Strong purifying selection operates on rDNA coding sequences (CDS) and hence identical or similar sequences may occur among different species (Nei \& Rooney 2005). Indeed, the nr18S clade-system proposed by Rowan \& Powers (1991) appears to grossly underestimate the actual taxonomic diversity within the Symbiodinium complex (Sampayo et al. 2009; LaJeunesse et al. 2010a; LaJeunesse \& Thornhill 2011). This limitation has been partially overcome by the development of high-resolution, rapidly evolving genetic markers, including two internal transcribed spacers of the rDNA cistron (ITS1 and ITS2; LaJeunesse 2001, 2002; Rodriguez-Lanetty 2003; Coffroth \& Santos 2005). In particular, sequence variation within the ITS2 region has revealed hundreds of sub-cladal phylotypes, particularly within the highly divergent clade C (Baker 2003; LaJeunesse 2005; Thornhill et al. 2013a). Recently, several studies have explored the fine-scale genetic diversity within these ITS2 phylotypes by analysing polymorphic microsatellite loci and other rapidly evolving genetic regions (Santos et al. 2003b; Magalon et al. 2006; Thornhill et al. 2013a; b, 2009, 2010; Kirk et al. 2009; Howells et al. 2009, 2013; Andras et al. 2011; Pettay et al. 2011; Baums et al. 2014). These studies show that geographically-defined population structure and host specificity often occur within widely distributed ITS2 populations, intimating the existence of yet another level of diversity beneath the subclade level. 


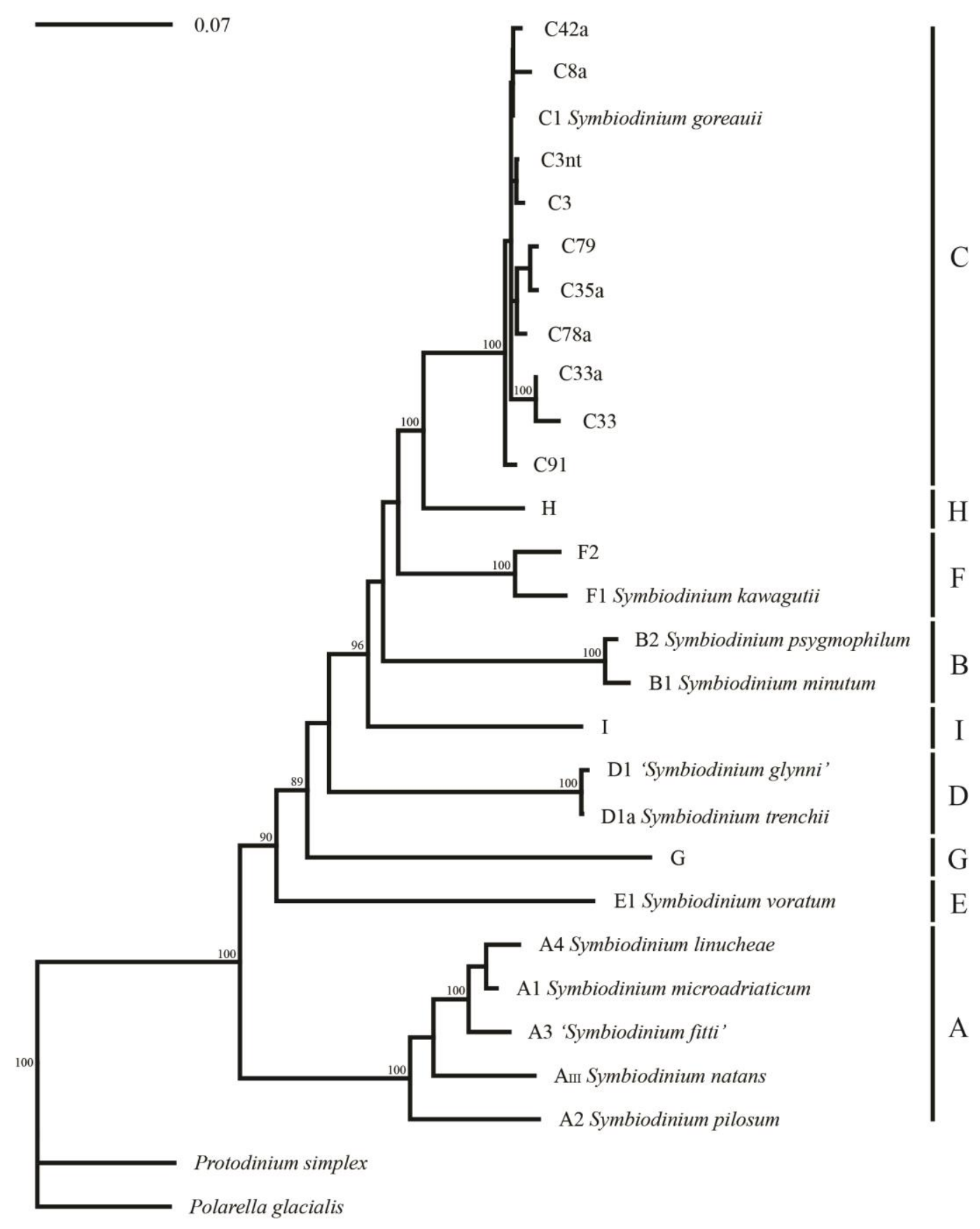

\section{Figure 1.4 Symbiodinium rDNA phylogeny}

Neighbour-joining tree of manually assembled rDNA concatenations of the partial $18 S$, complete ITS1, 5.8S and ITS2, and the D1-D2 domain of the 28S large ribosomal subunit gene (maximum 1534 bp), as would be amplified using the primers ITS1CLAMP (LaJeunesse et al. 2008) and 28S-reverse (Zardoya et al. 1995). The nucleotide alignment and tree were constructed in Geneious v7.0 (Biomatters) using the HKY five-parameter evolutionary model (Hasegawa et al. 1985), with Polarella glacialis and Protodinium simplex as outgroups. The scale bar indicates the number of nucleotide substitutions per sequence position. Numbers at branch nodes are consensus support values (\%) with those above $80 \%$ included in the figure. Each lineage is labelled using standard alphanumeric nomenclature (LaJeunesse 2001), and species names where applicable. Species in inverted commas lack formal description (nomen nudum; see Table 1.2). Large block letters indicate cladal membership (sensu Rowan \& Powers 1991). See Appendix A for Genbank accession numbers of sequences used in rDNA concatenations. 


\subsubsection{Functional diversity}

Divergent evolutionary trajectories have fostered extensive functional variation among many Symbiodinium taxa. This is evident in clade- and subclade-specific differences in photochemical efficiency (Savage et al. 2002; Rowan 2004; Goulet et al. 2005), growth rates (Kinzie et al. 2001), host infectivity (Schoenberg \& Trench 1980c; Davy et al. 1997; Xiang et al. 2013; Starzak et al. 2014), and in the quantity and quality of photosynthates translocated to the host (Markell \& Trench 1993; Loram et al. 2007b; Stat et al. 2008b; Cantin et al. 2009). Physiological and biochemical mechanisms underpinning these functional disparities include differences in chlorophyll distribution among chlorophyll-protein complexes (Iglesias-Prieto \& Trench 1997), variation in the regulation of photo-protective compounds (Banaszak et al. 2000), and differential lipid saturation in thylakoid membranes (Tchernov et al. 2004). The potential for dynamic photo-acclimation also varies between Symbiodinium taxa (Chang et al. 1983). Disparity in photo-physiological plasticity arises from differences in the regulation of chlorophyll-protein complexes (Iglesias-Prieto \& Trench 1994), the cycling of xanthophylls in non-photochemical quenching (Iglesias-Prieto \& Trench 1997), and in the ability to repair and/or replace the integral D1 protein of the photosystem II reaction centre (Warner et al. 1999; Ragni et al. 2010).

While the physiology of the host is important in determining its bleaching susceptibility (e.g. Fitt et al. 2009; Wicks et al. 2012; Paxton et al. 2013; Hawkins et al. 2014), the thermal adaptation of the symbiont can mean the difference between coral survival and mortality under stressful conditions (Baker et al. 2004; Rowan 2004; Berkelmans \& van Oppen 2006; Jones et al. 2008; Sampayo et al. 2008). Differences in temperature and light optima are evident both between and within clades, and there is a strong correlation between the thermal optimum of the symbiont and that of the host (Iglesias-Prieto \& Trench 1997; Warner et al. 1999; Savage et al. 2002; Rowan 2004; Tchernov et al. 2004; Goulet et al. 2005; Loram et al. 2007b; Sampayo et al. 2008; Fisher et al. 2011; Howells et al. 2011). The most striking example of symbiont thermal adaptation occurs in the Symbiodinium clade D species complex (S. trenchii, S. boreum, S. eurythalpos and S. glynni nomen nudum; LaJeunesse et al. 2014). While S. trenchii associates with many coral genera (LaJeunesse et al. 2009, 2010a), S. boreum and S. eurythalpos occur exclusively with Oulastrea crispata (LaJeunesse et al. 2014), and S. glynni is found only with Pocillopora spp. in the Indo-Pacific region (LaJeunesse et al. 2010b; Putnam et al. 2012). Despite their ecological differences, these symbionts can all confer a critical measure of thermal tolerance to their hosts under stressful 
conditions (LaJeunesse et al. 2014). In Guam, Pocillopora verrucosa colonies hosting $S$. glynni showed considerably higher photosynthetic efficiency than those hosting clade $\mathrm{C}$ at 32 ${ }^{\circ} \mathrm{C}$, while efficiencies were similar at $28.5{ }^{\circ} \mathrm{C}$ (Rowan 2004). Likewise, colonies of the brain coral Platygyra verwyei in close proximity to a hot water discharge from a nuclear power station in Taiwan exclusively harboured S. trenchii, with the prevalence of the heat-sensitive C3 gradually increasing with distance from the outlet (Keshavmurthy et al. 2012).

While Symbiodinium clade D represents a known thermo-tolerant lineage, there is otherwise a general lack of correlation between physiological performance and cladal membership (Savage et al. 2002). This is primarily due to the large amount of functional variability within clades, with sub-cladal congeners often showing pronounced differences in stress tolerance. For example, Stylophora pistillata colonies on the Great Barrier Reef (GBR) hosting symbiont types $\mathrm{C} 78$ or C8a were significantly less prone to thermal bleaching and mortality than those hosting C79 or C35a (Sampayo et al. 2008). Differences in thermal optima have even been reported between members of the same subclade. Temperature stress caused photo-damage and host-bleaching in a population of the subclade $\mathrm{C} 1$ (=S. goreauii) from a relatively cool region of the GBR, while a conspecific population from a warmer habitat remained relatively unaffected (Howells et al. 2011). Significant differences in thermal tolerance between minimally divergent populations suggest that functional adaptation may occur on small evolutionary time-scales, providing some optimism for the outlook of coral reefs in a rapidly changing climate (van Oppen et al. 2011; Howells et al. 2011).

\subsubsection{Ecological diversity}

The primary axis for niche diversification and speciation in Symbiodinium is the host taxon (LaJeunesse 2005). Host-symbiont combinations are conspicuously non-random, reflecting a complex co-evolution driven by cellular recognition and functional adaptation to the distinct, host-specific intracellular habitat (Trench 1997; Thornhill et al. 2013a). In a fine-scale genetic study of Symbiodinium phylotypes in the Caribbean, 32 out of 35 lineages within the subclade C3 were found to exclusively associate with a single host taxon (Thornhill et al. 2013a). Though host-symbiont fidelity appears to be the prevailing condition given current data, the degree of host-specificity can vary widely among symbiont taxa. For example, in a large-scale survey of symbiont diversity within the Indian Ocean, S. trenchii associated with 
26 out of 58 host genera in Thailand and 11 of 70 genera in Zanzibar (LaJeunesse et al. 2010a).

Functional differences between Symbiodinium types are also reflected in their spatial distribution patterns (Iglesias-Prieto \& Trench 1994, 1997; Toller et al. 2001a).

Environmental niche partitioning represents a secondary axis of diversification, and has been reported over latitudinal gradients (Loh et al. 2001; Rodriguez-Lanetty et al. 2001; Macdonald et al. 2008), between temperature microhabitats on a reef (Oliver \& Palumbi 2011; Keshavmurthy et al. 2012), over different depth ranges (Rowan et al. 1997; IglesiasPrieto et al. 2004; Sampayo et al. 2007; Frade et al. 2008), and between sites featuring distinct turbulence regimes (Ulstrup \& van Oppen 2003). The irradiance microhabitat even explains the vertical zonation of symbiont taxa within some individual coral colonies (Rowan \& Knowlton 1995; Rowan et al. 1997; van Oppen et al. 2001). However, S. trenchii again provides an exception to this general rule, showing a wide distribution across all major geographic regions (LaJeunesse et al. 2010a).

The lack of conformity by $S$. trenchii and other types to a single host taxon or habitat type can be explained by invoking the equilibrium-opportunist continuum as a third axis of diversification. Niche partitioning over both host-taxon and geographic/environmental axes is commonly observed in specialized types that show traits consistent with an 'equilibrium' life history. In particular, many phylotypes within Symbiodinium clade C exclusively associate with a single, vertically transmitting host species (LaJeunesse et al. 2003; Sampayo et al. 2007; Thornhill et al. 2013a). The resulting coevolution fosters a productive mutualism, characterized by highly beneficial nutritional reciprocity between symbiotic partners (Stat $e t$ al. 2008b; Cantin et al. 2009). Equilibrium taxa typically show a low affinity for physiological stress (Jones et al. 2008), and thus distribute within a narrowly defined habitat. On the other hand, 'opportunistic' types such as S. trenchii and S. minutum associate with multiple host taxa, exhibit a high tolerance of physiological stress, and show a widespread but patchy distribution (Toller et al. 2001b; LaJeunesse 2005; Stat et al. 2008b; LaJeunesse et al. 2009, 2010a). A trait commonly associated with an opportunistic life history involves a reduction in nutritional value to the host (Cantin et al. 2009), sometimes to the point of marginal mutualism (Stat et al. 2008b). The disturbance created by bleaching and disease can facilitate 'outbreaks' by opportunistic symbionts, leading to conspicuous shifts in dominance across the reef (Baker et al. 2004; LaJeunesse et al. 2009, 2010b; Correa \& Baker 2011). This is generally a transient state, with return of conditions to pre-stress levels facilitating the 
succession of equilibrium taxa (Thornhill et al. 2006b; Jones et al. 2008; LaJeunesse et al. 2009, 2010b).

\subsection{Symbiodinium systematics}

\subsubsection{Assigning species nomenclature}

The Symbiodinium genus clearly represents a genetically, functionally and ecologically diverse complex. Assigning binomial species nomenclature to the many different 'types' is an important endeavour for comparing, aligning and integrating global research efforts, and evaluating conservation status (Green 2005). Yet efforts to formally describe Symbiodinium species are hampered by morphological similarities between divergent phylotypes, and their primarily asexual mode of reproduction (Sampayo et al. 2009; Stat et al. 2012; LaJeunesse et al. 2012). Alternatives to the classic biological species concept (also known as the isolation species concept; Mayr 1942, 1963) such as the ecological species concept (ESC; van Valen 1976; Andersson 1990) and the cohesion species concept (CSC; Templeton 1989) provide suitable criteria by which to delineate Symbiodinium species (Correa \& Baker 2009; Thornhill et al. 2013a). Both provide faculty for clonal or predominantly clonal lineages, whose species candidacy cannot be assessed on the basis of reproductive isolation (Mayr 1999). However, the delineation of species boundaries using both sets of criteria hinge on building well-supported phylogenetic trees based on molecular sequence data.

\subsubsection{The internal transcribed spacer 2 (ITS2)}

The ITS 2 has emerged as the most extensively utilised marker for resolving fine-scale Symbiodinium systematics (Franklin et al. 2012; Tonk et al. 2013), and can provide taxonomic resolution roughly corresponding to the species level (LaJeunesse 2001). This marker can be used in conjunction with denaturing gradient gel electrophoresis (DGGE) to provide a relatively fast, cost-effective and reproducible means of establishing the dominant Symbiodinium phylotype(s) within a coral colony (LaJeunesse \& Trench 2000; LaJeunesse 2001, 2002). However, ITS2 sequence diversity may not always provide an accurate reflection of species diversity. Sequence variation in the ITS2 region may provide a level of resolution that is too coarse to distinguish species within the more derived Symbiodinium 
lineages such as $\mathrm{C} 1$ and $\mathrm{C} 3$, necessitating the use of more rapidly evolving markers (Thornhill et al. 2013a). In other lineages, phylogenetic inference based on ITS2 variation can result in inflated diversity estimates. For example, Litaker et al. (2007) showed that intergenomic ITS sequence variation occurs within several well-defined species of free-living dinoflagellates. A cluster-based approach has been proposed to control for diversity inflation in Symbiodinium (Correa \& Baker 2009); yet this method merely highlights groups of ITS2 phylotypes that require further genetic, physiological and ecological analysis (Stat et al. 2012).

The multiple-copy nature of rDNA imposes a further limitation on the phylogenetic utility of the ITS2. rDNA genes in dinoflagellates (and most other eukaryotes) are arranged in extensive tandem arrays (Hackett et al. 2004; Zhang et al. 2006; Figure 1.5a), with some free-living species hosting chromosomes almost entirely composed of rRNA repeats (Figueroa et al. 2014). A mutation occurring within an rDNA repeat may be eliminated, or promoted to fixation throughout the genome via stochastic DNA repair/replication processes that stimulate gene conversion or duplication/deletion. These processes include unequal crossover during meiosis (Figure 1.5b), transposition, retrotransposition, slipped-strand mispairing and intra-strand exchange within and between chromatids (reviewed in Nei \& Rooney 2005). The effect of these processes is to homogenize intra-genomic sequence variation and cause repetitive genetic elements to evolve in concert ('concerted evolution'; Brown et al. 1972; Arnheim et al. 1980; Figure 1.5c) While concerted evolution acts to eliminate intra-genomic sequence variation, mutations continuously arise during DNA replication and repair (paralogs; Hou \& Lin 2009). It may take many generations for a mutated rDNA copy to be eliminated from the genome or to reach fixation, particularly if ribosome-coding genes occupy chromosomal positions near the centromere (Cronn et al. 1996). Hence a non-diagnostic polymorphism (NDP) may remain within a lineage for extended periods of evolutionary time (Álvarez \& Wendel 2003). The intra-genomic persistence of NDPs can yield false diversity signals and lead to incorrect phylogenetic inference, particularly when detected in plasmid cloning assays of bulk-cell Symbiodinium samples (van Oppen \& Gates 2006; LaJeunesse \& Pinzón 2007; Thornhill et al. 2007; Miranda et al. 2012). Single-cell PCR (scPCR) offers a solution, controlling for inflated diversity estimates and providing increased accuracy in phylogenetic reconstruction (Miranda et al. 2012). However, the difficult and time-consuming nature of this technique has prevented its widespread utility in Symbiodinium systematics. 
(a)

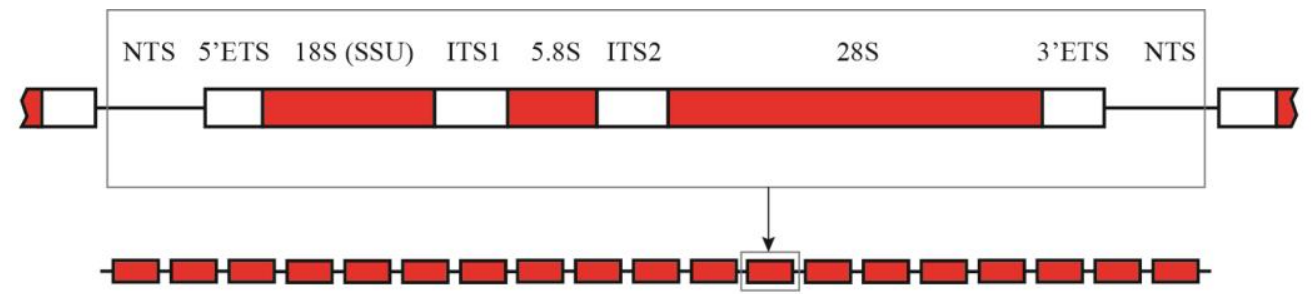

(b)

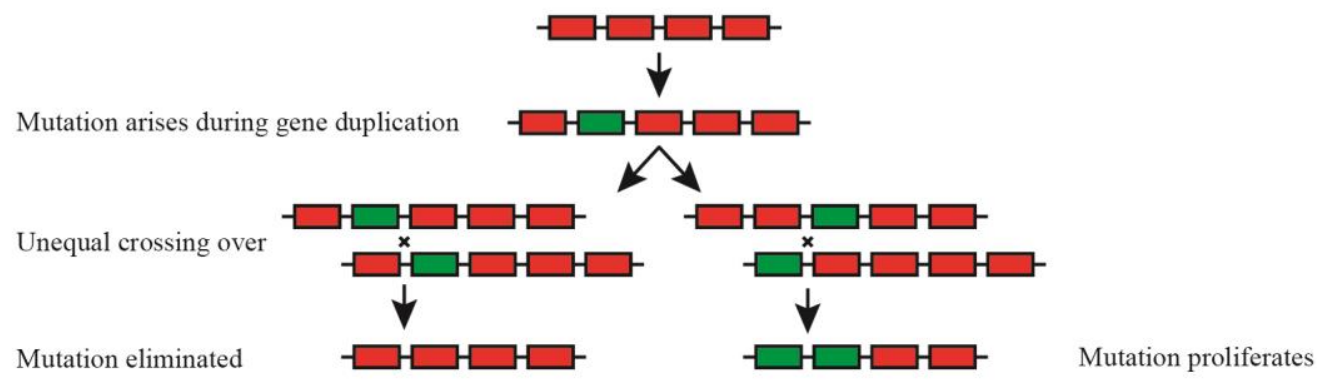

(c)

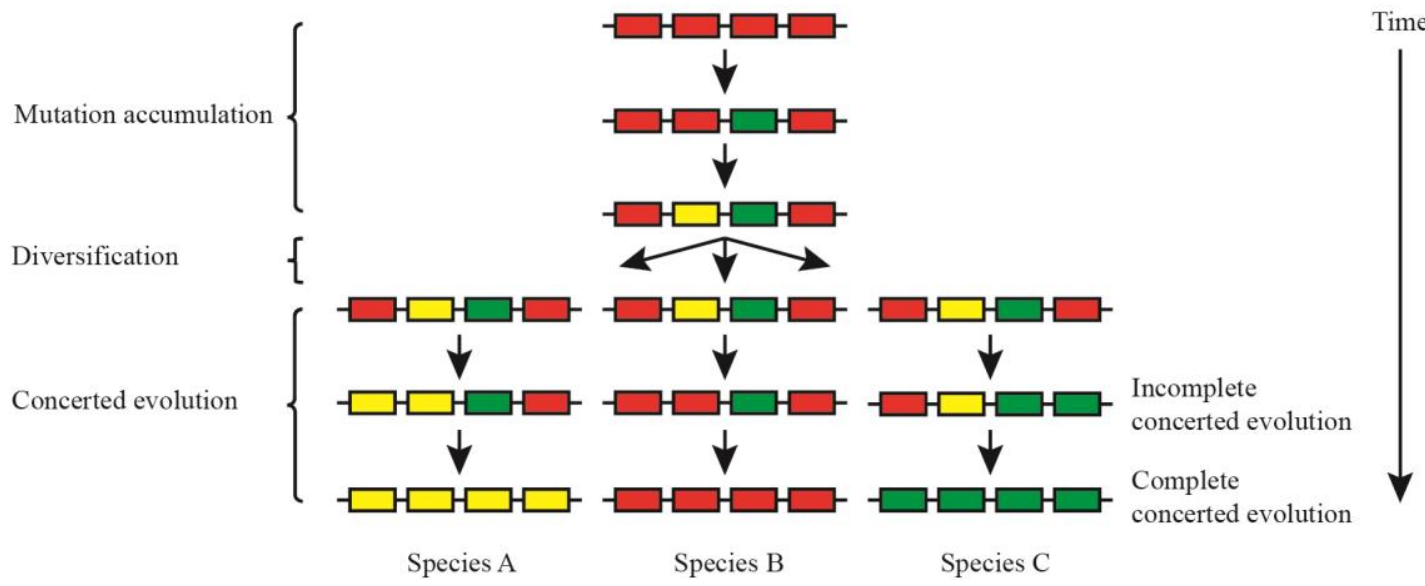

\section{Figure 1.5 Concerted evolution of rDNA in the nuclear genome}

The eukaryote nuclear genome contains rDNA genes in tandem arrays (a), with copy numbers ranging from less than 100 to several hundred thousand. Each rDNA repeat comprises two external transcribed spacers (5'ETS and 3'ETS), a small subunit gene (SSU; 18S), two internal transcribed spacers (ITS1 and ITS2), and the 5.8S and 28S genes that collectively code for the large ribosomal subunit (LSU). Each tandem repeat is separated from its neighbour by a non-transcribed spacer (NTS). A mutation may occur in an rDNA repeat during gene duplication or repair (b; mutated copy shown in green), and the resulting polymorphism may either dissipate from the genome or ascend to fixation through concerted evolutionary processes such as unequal crossing-over during meiosis. Concerted evolution can take many generations to homogenize all copies within the genome (c; polymorphic copies shown in different colours). When concerted evolution is complete, rDNA arrays are useful for inferring phylogenetic relationships; however when arrays are in a state of incomplete homogenization (e.g. following divergence or hybridization), rDNA markers can yield ambiguous sequence reads, inflated diversity estimates and incorrect phylogenies (Buckler et al. 1997; Álvarez \& Wendel 2003). 


\subsubsection{Alternative genetic markers}

The limitations accompanying the use of the ITS2 for phylogenetic inference have prompted assessments of several alternative candidate markers. Yet each has their drawbacks and a general consensus on a benchmark 'species' level marker for Symbiodinium has yet to be reached. The nuclear-encoded actin gene provides an acceptable level of resolution (Pochon et al. 2012) and has seen limited utility (Mieog et al. 2009; Cunning et al. 2013; Cunning \& Baker 2013); however it occurs in multiple copies and appears to follow the 'birth and death' model of evolution, thus facilitating the accumulation of intra-genomic variation arising from the degeneration of pseudo-genes (Kim et al. 2011). Indeed, 10 of the 46 actin genes in the genome of the free-living dinoflagellate Amphidinium carterae were identified as pseudogenes (Bachvaroff \& Place 2008). Despite this, the actin copy number is suitably low in Symbiodinium, estimated at 7 in clade $\mathrm{C}$ and just a single copy in clade D (Mieog et al. 2009). This is considerably lower than the ITS2, which numbers in the hundreds to tens of thousands of copies per Symbiodinium genome (Mieog et al. 2009). Length heteroplasmy in a hyper-variable area of domain $\mathrm{V}$ in the $c p 23 S$ plastid rDNA can be assessed using polyacrylamide gel electrophoresis (PAGE) to rapidly identify dominant and coexisting symbiont types at the sub-cladal level (Santos et al. 2003a). However, while useful in screening taxa within clades A and B, this technique is not sufficiently sensitive to resolve the more recently-diverged members of clade C (Sampayo et al. 2009). Alternatively, the non-coding region of the plastid-encoded photosystem II D1 protein gene ( $p s b A^{n c r}$ ) can show substantially higher rates of divergence than either the ITS2 or the cp23S (Thornhill et al. 2013a), while generally maintaining low copy numbers (but see Koumandou \& Howe 2007) and similarly low intra-genomic variation (Moore 2003; LaJeunesse \& Thornhill 2011). However, universal Symbiodinium primers are not available for $p s b A^{n c r}$, and hence specific primers must be tailored for individual clades. Microsatellite flanking regions such as Sym15 and Si4.86 also show high sensitivity and have the added advantage of occurring in a singlecopy per genome (Santos et al. 2004; Pettay \& LaJeunesse 2007). By targeting these markers, Finney et al. (2010) showed that the ITS2 lineage B1 (or cp23S lineage B184) consists of a diverse assemblage of putative species. Yet similar to the $p s b A^{n c r}$, all microsatellite flanking regions characterized to date require primers that are clade- or even subclade-specific. The mitochondrial-encoded (mtDNA) cytochrome oxidase genes cob (Zhang et al. 2005) and coI (or coxl; Takabayashi et al. 2004) have also been used in Symbiodinium systematics. A large and expanding dataset of $c o I$ sequences is available through the 'barcode of life' project 
(www.barcoding.si.edu), making this marker particularly appealing (Stern et al. 2010). However, mtDNA genes tend to be highly conserved in dinoflagellates, obscuring fine-scale diversity (Takabayashi et al. 2004; Zhang et al. 2005; Sampayo et al. 2009; Pochon et al. 2014).

\subsubsection{A consensus on Symbiodinium systematics?}

Despite promising progress in the development of alternative markers, the rDNA cistron remains the benchmark for Symbiodinium systematics. In a recent evaluation of candidate markers, the Symbiodinium rDNA phylogeny resolved that of the combined multi-gene concatenation better than any of the plastid or mitochondrial genes assessed (cp23S, $p s b A^{n c r}$, coI and cob; Pochon et al. 2014). This may reflect well-documented errors in organelle phylogenies that arise from lineage sorting, organelle capture and hybridization (Rieseberg \& Soltis 1991; Rieseberg et al. 1996; Tsitrone et al. 2003). For these and other reasons, the 'barcode of life' consortium recommends that rDNA markers are more appropriate than mtDNA or chloroplast DNA (cpDNA) for systematic barcoding in protists (Pawlowski et al. 2012).

In practice, the most suitable gene(s) to target depends on the taxonomic question being addressed (Pochon et al. 2012, 2014). To formally describe Symbiodinium species, an agreeing phylogenetic topology from a combination of coding and non-coding nuclear, mitochondrial, and chloroplast sequences, as well as corroborative morphological, physiological and ecological data are necessary (Sampayo et al. 2009; LaJeunesse et al. 2012).To date, twelve Symbiodinium species have been described (Table 1.1) and a further five have been assigned tentative species names (nomena nuda; Table 1.2). Yet the vast majority of diversity within this genus awaits taxonomic description (LaJeunesse et al. 2012). Several recent advances in molecular methodology facilitate progress in this area. These include the assembly and public availability of the Symbiodinium nuclear and plastid genomes (Shoguchi et al. 2013; Barbrook et al. 2014), the increased accessibility and utility of high-throughput sequencing platforms (Quigley et al. 2014; Thomas et al. 2014), and the centralization of multi-gene sequence data within comprehensive databases (Franklin et al. 2012; Tonk et al. 2013). Such developments will likely be of considerable benefit in the effort to characterize new molecular markers, assign species nomenclature, establish diversity estimates, and reconstruct the evolutionary history of the Symbiodinium complex. 
Table 1.1 List of formally described Symbiodinium species

\begin{tabular}{|c|c|c|c|c|}
\hline Species name & Synonyms & ITS2 type & Host (holotype) & Reference \\
\hline Symbiodinium boreum & & D15 & Oulastrea crispata & (LaJeunesse et al. 2014) \\
\hline Symbiodinium eurythalpos & & $\begin{array}{l}\text { D8, D8-12, D12- } \\
13, \text { D13 }\end{array}$ & Oulastrea crispata & (LaJeunesse et al. 2014) \\
\hline Symbiodinium goreauii & S. goreaui & $\mathrm{C} 1$ & Ragactis lucida & (Trench \& Blank 1987; Trench 2000) \\
\hline Symbiodinium kawagutii & & $\mathrm{F} 1$ & Montipora verrucosa & (Trench \& Blank 1987; Trench 2000) \\
\hline Symbiodinium linucheae & Gymnodinium linucheae & A4 & Linuche unguiculata & (Trench \& Thinh 1995) \\
\hline Symbiodinium microadriaticum & & A1 & Cassiopeia xamachana & (Freudenthal 1962) \\
\hline Symbiodinium minutum & & B1 & Aiptasia sp. & (LaJeunesse et al. 2012) \\
\hline Symbiodinium natans & & A & Free-living (Tenerife) & (Hansen \& Daugbjerg 2009) \\
\hline Symbiodinium pilosum & S. meandrinae, $S$. corculorum & $\mathrm{A} 2$ & Zoanthus sociatus* & (Trench \& Blank 1987; Trench 2000) \\
\hline Symbiodinium psygmophilum & & $\mathrm{B} 2$ & Oculina diffusa & (LaJeunesse et al. 2012) \\
\hline Symbiodinium trenchii & S. trenchi & D1a & Unspecified & (LaJeunesse et al. 2014) \\
\hline Symbiodinium voratum & S. californium, S. varians & E1 & Free-living (South Korea) & (Jeong et al. 2014) \\
\hline
\end{tabular}

*This holotype may not represent the dominant symbiont of $Z$. sociatus, since $S$. pilosum is not known to form endosymbiosis with other cnidarians

(LaJeunesse 2002) and free-living types are known to out-compete and over-grow symbiotic strains in culture (Santos et al. 2001).

Table 1.2 List of Symbiodinium species lacking formal description (nomen nudum)

\begin{tabular}{|c|c|c|c|c|}
\hline Species name & Synonyms & ITS2 type & Host & Reference \\
\hline Symbiodinium bermudense & S. pulchrorum & B1 & Aiptasia pallida, A. pulchella & (Banaszak et al. 1993) \\
\hline Symbiodinium cariborum & $\begin{array}{l}\text { S. microadriaticum subsp. } \\
\text { Condylactis }\end{array}$ & A1.1 & Condylactis gigantea & (Blank \& Huss 1989; Banaszak et al. 1993) \\
\hline Symbiodinium fitti & & A3 & Acropora spp., Tridacna spp. & (Pinzón et al. 2011) \\
\hline Symbiodinium glynni & & D1 & Pocillopora spp. & (LaJeunesse et al. 2010b) \\
\hline Symbiodinium muscatineii & S. muscatinei & B4 & Anthopleura elegantissima & (LaJeunesse \& Trench 2000) \\
\hline
\end{tabular}




\subsection{Coral-symbiont adaptation and acclimatization}

\subsection{1. 'Adaptive' bleaching}

The realization that genetic, functional and ecological diversity exists within the Symbiodinium complex prompted the formulation of the somewhat contentious 'adaptive bleaching hypothesis' (ABH; Buddemeier \& Fautin 1993). The ABH postulated that, rather than representing an ominous threat to coral reefs, bleaching might serve as an adaptive mechanism by which corals could survive periods of climatic upheaval. According to the $\mathrm{ABH}$, coral bleaching "provides an opportunity for the host to be repopulated by a different type of partner", and altered environmental conditions "favour establishment of combinations of symbionts that were less adaptive under previous conditions" (Buddemeier \& Fautin 1993). A central assumption of the ABH in its original form was that established coral colonies can switch to a new symbiont phylotype obtained from an environmental source population (Kinzie et al. 2001). 'Symbiont switching' (Figure 1.6a) has been induced in the laboratory in anemones, octocorals and scleractinians (e.g. Schoenberg \& Trench 1980; Davy et al. 1997; Kinzie et al. 2001; Lewis \& Coffroth 2004; Coffroth et al. 2010). However, the experimentally manipulated host-symbiont associations in corals are generally transient, reverting to the original combination over time (Coffroth et al. 2010). Furthermore, there remains no evidence that a novel symbiont can colonize and ascend to dominance within a coral after a natural bleaching event. A post-bleaching change in dominant symbiont was reported as evidence of 'adaptive bleaching' in transplanted coral colonies (Baker 2001); however this interpretation drew criticism. In particular, Hoegh-Guldberg et al. (2002) pointed out that Baker had not shown evidence of adaptation, but rather an acclimatization mechanism, one of many that corals have at their disposal to cope with environmental stress (e.g. Falkowski \& Dubinsky 1981; Palumbi et al. 2014). Fautin \& Buddemeier (2004) provided semantic clarification stating that 'adaptation' was intended in the English sense, defined as "modification of an organism or its parts in a way that makes it more fit for existence under the conditions of its environment", thereby extending its definition to include acclimation and acclimatization (note that this definition differs fundamentally from that used in evolutionary biology, in which adaptive modification is heritable, and therefore genetically encoded; Dobzhansky 1968). The concept of adaptive bleaching was thus generalized to include an acclimatory change in the relative proportion of pre-existing types (i.e. 'symbiont shuffling'; Figure 1.6b and c), and extended to encompass the entire continuum from 
catastrophic bleaching to visually undetectable symbiont turnover (Fautin \& Buddemeier 2004).

\subsubsection{Mixed symbiont populations and symbiont shuffling}

Until recently, mixed Symbiodinium infections were thought to occur in only a minority of coral species (Diekmann et al. 2002; Goulet 2006). However, modern ultra-sensitive molecular techniques such as qPCR, fluorescence in situ hybridization (FISH) and next generation sequencing (NGS) increasingly reveal cryptic symbiont populations (Baker \& Romanski 2007; Loram et al. 2007a; Mieog et al. 2007; Correa et al. 2009; Silverstein et al. 2012; Quigley et al. 2014; Thomas et al. 2014). For example, Silverstein et al. (2012) surveyed the symbiont consortia of 39 coral species (including 26 'symbiont specialists') using a clade-specific qPCR assay, and found representatives from all species that hosted at least two Symbiodinium clades. Within-colony symbiont diversity remains particularly unexplored in Indo-Pacific corals, many of which simultaneously host multiple, closely related types within clade C (Baker 2003; LaJeunesse et al. 2004; LaJeunesse 2005; Baker \& Romanski 2007; Stat et al. 2011).

Theoretical virulence models predict that mixed symbiont infections should be less mutualistic than monoclonal populations, due to the tendency of related symbionts to compete for host resources (van Baalen \& Sabelis 1995; Frank 1996b). Yet there remains no evidence of direct antagonism between symbionts within a cnidarian host (Dunn \& Weis 2009). Furthermore, if mixed infections promote symbiotic disruption, selection should favour vertical transmission (Douglas 1998), whereas only around 15-30\% of coral species transmit symbionts from parent to offspring (Fadlallah 1983; Harrison \& Wallace 1990; Richmond \& Hunter 1990; Baird et al. 2009). Moreover, the limited empirical evidence specifically addressing symbiont competition in cnidarians suggests that mixed infection is not synonymous with symbiotic disruption. In a study tracking the fate of photosynthetically fixed carbon in the giant sea anemone Condylactis gigantea, Loram et al. (2007b) showed that the amount of translocated photosynthate was simply an additive function of the specific productivity and relative abundance of each of the types present (Symbiodinium clades A and B), indicating that their coexistence did not have negative implications for the host. Symbiont competition therefore appears to be predominantly or exclusively under the auspices of hostregulation. This control may be exerted through symbiont compartmentalisation (Correa \& 
Baker 2011), within-colony niche partitioning (Rowan \& Knowlton 1995; Rowan et al. 1997), and/or the regulation of symbiont biomass via selective expulsion/degradation, nutrient limitation and chemical signalling (see Davy et al. 2012 for a review). The persistence of mixed infections despite host regulation suggests that, rather than promoting virulence, this condition may actually confer a selective advantage under certain conditions.

Mixed symbiont infections may benefit the host by providing an 'insurance policy' (sensu Yachi \& Loreau 1999), conferring resilience against abiotic stress by increasing the capacity to maintain function in the face of environmental change (Nyström 2006). If the dominant symbiont is suddenly rendered ill-adapted to a modified environment, a cryptic, more suitably-adapted type may take its place, thus enabling the host to survive (Baker 2003). Dominance shifts have been well-documented within the symbiont consortia of corals and anemones following changes in their environment (Rowan et al. 1997; Baker 2001; Toller et al. 2001b; Chen et al. 2005; Berkelmans \& van Oppen 2006; Thornhill et al. 2006b; Jones et al. 2008; Sampayo et al. 2008; Venn et al. 2008; Jones \& Berkelmans 2010). In the majority of cases, a symbiont change was stimulated by bleaching, with the disturbance facilitating the proliferation of a previously cryptic population (Figure 1.6b). For example, Berkelmans \& van Oppen (2006) transplanted colonies of Acropora millepora to an area featuring a higher average temperature, and observed a post-bleaching change in the dominant symbiont type from the naturally present clade $\mathrm{C}$ in favour of clade D. Repopulation by Symbiodinium D conferred a $1-1.5{ }^{\circ} \mathrm{C}$ increase in heat tolerance, and enabled affected colonies to survive the disturbance (Berkelmans \& van Oppen 2006).

Though not as well-characterized as bleaching-induced symbiont shuffling, changes within the symbiont consortium can also occur in the absence of bleaching (dynamic symbiont shuffling; Figure 1.6c). In this case, succession occurs through gradual symbiont turnover, effected by differential elimination and regeneration (Yamashita et al. 2010). In many horizontal-transmitting species, symbiont succession is evident during the early stages of ontogeny, prior to the establishment of more stringent symbiont-specificity (Coffroth et al. 2001; Little et al. 2004). Dynamic symbiont shuffling also occurs in disease-affected adult corals, possibly arising from compromised immunity (Toller et al. 2001b), and in healthy adults in response to natural environmental variation (Chen et al. 2005; Venn et al. 2008). 
(a)

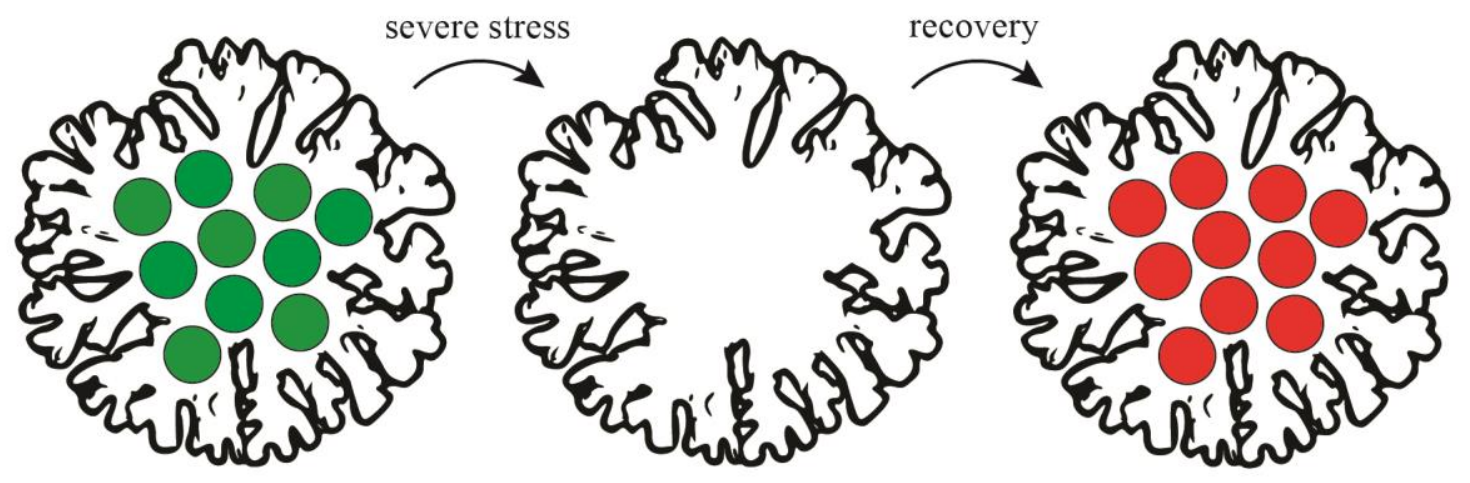

(b)

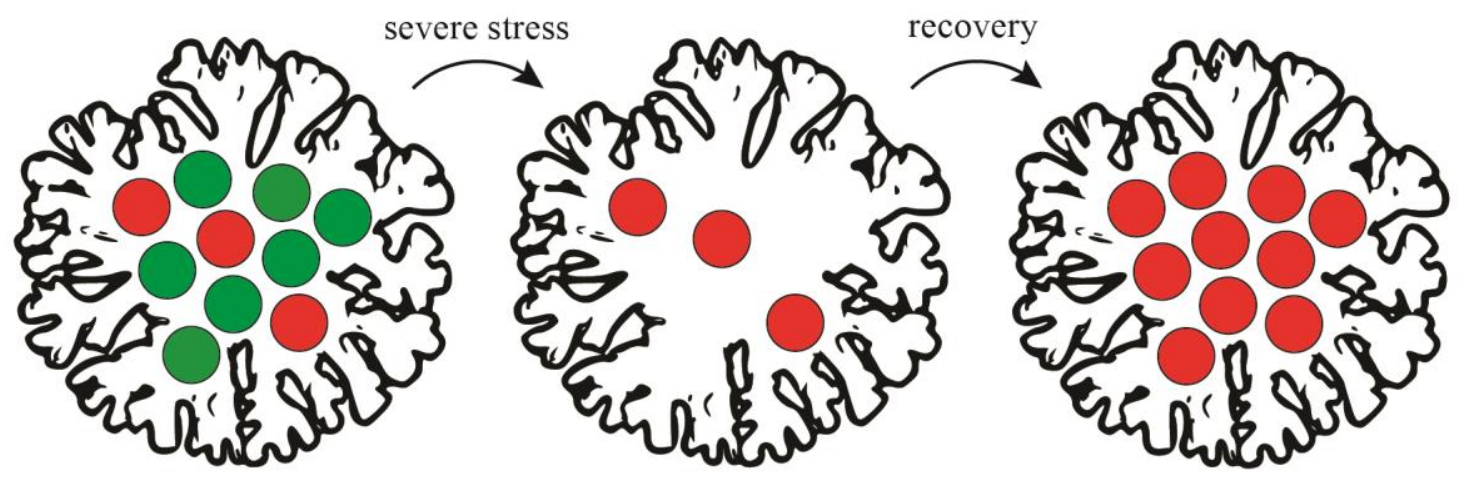

(c)

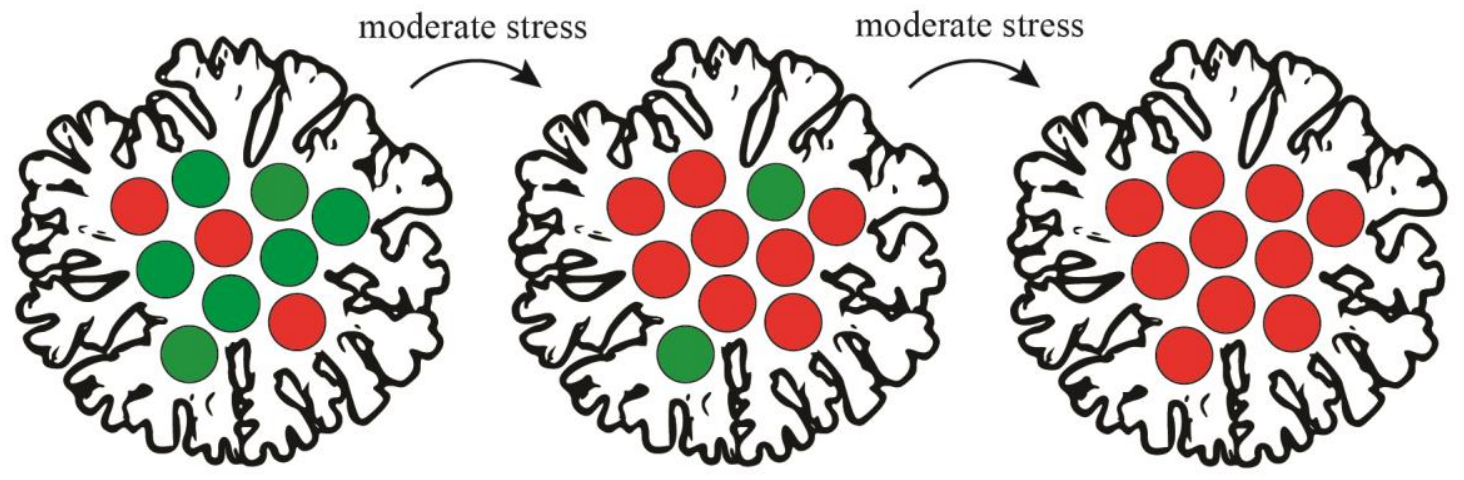

Figure 1.6 Symbiont switching and shuffling

A change in symbiont dominance within a coral colony from a heat-sensitive type (green) to a heat tolerant type (red) can occur via (a) a switch to a new symbiont from an environmental source population following bleaching-related disturbance; (b) the post-bleaching proliferation of a residual, thermally tolerant type (bleaching-induced symbiont shuffling); or (c) gradual symbiont turnover in response to environmental change (dynamic symbiont shuffling). Symbiont 'switching' has not been documented in the natural environment. 
While symbiont shuffling appears to offer improved host benefit relative to symbiont switching, optimistic predictions for corals in the face of warming temperatures are accompanied by several major caveats. First, similar to symbiont switching, host-symbiont associations modified by symbiont shuffling appear to be transient, and long-term follow up studies generally reveal post-bleaching reversion (Thornhill et al. 2006b; Jones et al. 2008; LaJeunesse et al. 2009; but see Sampayo et al. 2008). Second, symbiont shuffling may involve severe physiological trade-offs for the host. Opportunistic symbionts that proliferate within the tissue of corals after bleaching may offer comparatively less nutritional value (Stat et al. 2008b; Cantin et al. 2009), resulting in slower coral growth rates (Little et al. 2004; Jones \& Berkelmans 2010) and increased susceptibility to disease (Stat et al. 2008b). Surviving corals may therefore suffer drastic reductions in fecundity, and take many years to fully recover (Hoegh-Guldberg 1999). Third, it is not known how many coral species are able to 'shuffle' symbionts. While the symbiont consortium is dynamic in some species, hostsymbiont co-evolution has fostered rigid fidelity in others (Stat et al. 2006; Putnam et al. 2012). This is evident in several fine-scale population studies that reveal the multi-year persistence of a single clonal genotype within individual colonies (Goulet \& Coffroth 2003a; Kirk et al. 2005; Thornhill et al. 2006a, 2009; Andras et al. 2011; Pettay et al. 2011). In particular, vertical-transmitting species characteristically show a reduced affinity for symbiont shuffling (Thornhill et al. 2006a). This is highlighted by the disproportionate number of studies reporting symbiont shuffling in horizontal-transmitting species (Rowan et al. 1997; Coffroth et al. 2001; Baker 2001; Toller et al. 2001b; Little et al. 2004; Chen et al. 2005; Berkelmans \& van Oppen 2006; Thornhill et al. 2006b, 2009; Jones et al. 2008; Venn et al. 2008; Jones \& Berkelmans 2010; but see Sampayo et al. 2008). Symbionts are vertically-transmitted in around 15-30\% of scleractinian coral species (Baird et al. 2009), suggesting that symbiont shuffling may not be a universally available acclimatory mechanism. Finally, symbiont shuffling appears to offer a maximum increase in thermal tolerance of around $1-1.5^{\circ} \mathrm{C}$ (Berkelmans \& van Oppen 2006). Given that recent model simulations predict a $1.5-3{ }^{\circ} \mathrm{C}$ increase by the year 2050 (Kirtman et al. 2013), symbiont shuffling will unlikely mitigate the environmental stress that corals are expected to face in the near future. Therefore, while 'adaptive bleaching' sensu lato has provided a source of optimism for the outlook of corals in the face of warming ocean temperatures (Baker 2001; Fautin \& Buddemeier 2004; Berkelmans \& van Oppen 2006), the persistence of corals in a changing climate will ultimately depend on the ability of both symbiotic partners to acquire functional adaptations to cope with abiotic stress. A shift in focus towards evolutionary 
adaptation sensu stricto is clearly necessary if we are to gain a realistic insight into the future of coral reefs.

\subsection{Symbiodinium evolution}

\subsubsection{Asexual reproduction in Symbiodinium}

The classic model of Symbiodinium evolution depicts an asexual, permanent haploid lineage that diversifies through host-specialization and geographic isolation (LaJeunesse 2005). Genetic variation accumulates through somatic mutation, and similar to asexual prokaryotic lineages, evolution is characterised by punctuated speciation events and 'selective sweeps'(LaJeunesse 2005; Correa \& Baker 2009). This is consistent with theoretical models that predict a higher affinity for asexual reproduction in mutualistic symbionts than in pathogens, whose inclination towards sexual reproduction is driven by a continuous evolutionary 'arms race' with their hosts (Law \& Lewis 1983; Maynard Smith 1998). The proposed loss of sexual reproduction in Symbiodinium has led some authors to conclude that the evolutionary responsiveness within this lineage is likely minor (Buddemeier \& Fautin 1993). However, the short generation times observed in symbiosis (Wilkerson et al. 1988) and the high frequency at which somatic mutations occur (van Oppen et al. 2011) suggest that rapid adaptation could occur during periods of environmental change. This is exemplified by the free-living asexual dinoflagellate Prorocentrum triestinum, in which somatic mutations alone conferred a $5{ }^{\circ} \mathrm{C}$ increase in thermal tolerance within around 400 generations (Flores-Moya et al. 2008).

While somatic mutations could explain the broad genetic and functional diversity within the Symbiodinium complex, exclusive clonality presents a paradox when one considers the long evolutionary persistence of this lineage (over 50 million years; Tchernov et al. 2004). With very few exceptions (e.g. bdelloid rotifers, darwinuloid ostracods and arbuscular mycorrhizal fungi), the loss of sexual reproduction condemns lineages to early extinction (Judson \& Normark 1996). Muller's ratchet dictates that the gradual, irreversible accumulation of deleterious somatic mutations (genetic load) in an asexual population inevitably leads to the erosion of mean fitness over time (Felsenstein 1974). Genetic information is eventually lost through stochastic genetic drift, and high-fitness genotypes are successively replaced by deleterious mutants (Crow 1994). It is thought that mutation accumulation can only truly be 
overcome by meiotic recombination, explaining the persistence of sexual reproduction despite its high cost in comparison to clonality (Muller 1964). Haploidy can lessen the speed of the ratchet since the mutation rate within the genome is effectively halved and deleterious alleles are always expressed (and hence exposed to stronger selection). However, haploids are not immune to 'ratchet clicks', even when population sizes are large (Gordo \& Charlesworth 2000). Highly efficient DNA repair mechanisms in some asexual lineages have been implicated in reducing genetic load (Schön \& Martens 1998). In particular, ameiotic recombination via reciprocal crossover or non-reciprocal gene conversion can reduce the accumulation of deleterious alleles in asexual diploid crustaceans (Daphnia spp.; Omilian et al. 2006) and tetraploid rotifers (Adienta vaga; Flot et al. 2013). Yet in the absence of homologous chromosomes to use as templates for gene-conversion, how has genetic load not led to the extinction of the haploid, asexual Symbiodinium lineage?

\subsubsection{Sexual reproduction in Symbiodinium}

In his initial species description of Symbiodinium microadriaticum, Freudenthal (1962) reported putative cysts with developing stages resembling isogametes. Small zoospores resembling gametes were also observed by Taylor $(1973,1974)$, and Schoenberg \& Trench (1980a), but the critical processes of fusion and recombination remained elusive. These observations, in addition to their own, led Fitt \& Trench (1983) to form the hypothesis that the Symbiodinium life cycle includes a sexual stage. Specifically, their hypothesis suggested that haploid zoospores fuse and undergo plasmogamy to form a transient diploid zygote, which then undergoes meiosis to restore the haploid condition (i.e. a haplontic life cycle; Figure 1.7). This is consistent with the majority of free-living dinoflagellates, nearly all of which are haplontic (Pfiester \& Anderson 1987; Pfiester 1989; Elbrächter 2003).

The initial molecular evidence that Symbiodinium undergoes cryptic meiotic recombination came from isoenzyme analyses. Incongruence between isoenzyme phylogenies and those constructed from both RAPD (Baillie et al. 1998, 2000) and ITS2 sequence variation (LaJeunesse 2001) implicated allelic recombination, consistent with criteria outlined to distinguish between clonal and sexual eukaryote populations (Tibayrenc et al. 1991). More recently, meiotic recombination has been inferred from linkage disequilibrium between microsatellite loci (Santos et al. 2003b; Pettay et al. 2011; Thornhill et al. 2013a; LaJeunesse et al. 2014; Baums et al. 2014), indicating that extensive shuffling of alleles has occurred 
within several Symbiodinium lineages. Additionally, a recent meta-genomic analysis revealed the presence of six meiosis-specific and 25 meiosis-related functional genes in published Symbiodinium genomes (Chi et al. 2014), suggesting that the loss of sexual reproduction has not occurred in this genus.

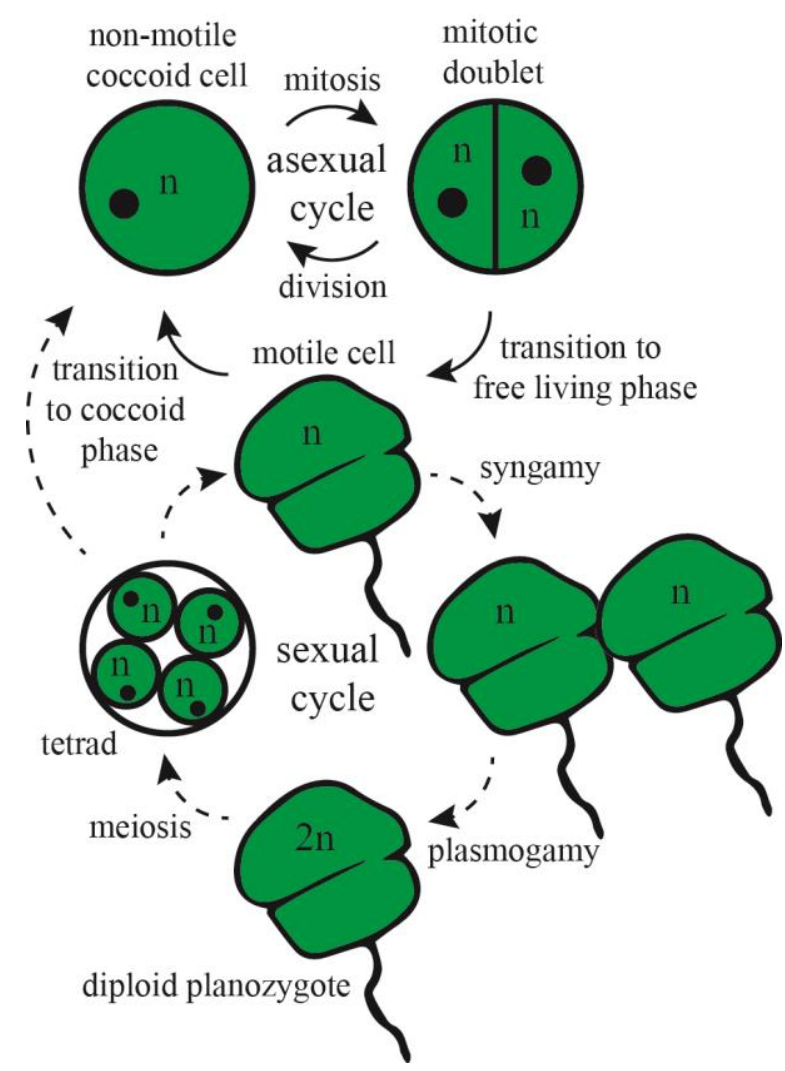
Figure 1.7 The putative haplontic life- cycle of Symbiodinium

Closed arrows show observed processes and dashed arrows represent those that are inferred. While the sexual life cycle has not been directly observed, cryptic recombination is evident from several multi-locus genotyping studies (see below). Symbiodinium cells are coccoid and non-motile when in symbiosis (black circles represent accumulation bodies, visible under the light microscope) and transition to the flagellate motile form when in the freeliving phase. Coccoid and flagellate cells are primarily haploid (n). The putative zygote is diploid ( $2 n$ ) before undergoing meiosis to restore the haploid condition (modified from Stambler 2011).

While symbiotic dinoflagellates do appear to undergo cryptic recombination, it remains unknown when and where sexual reproduction occurs. The Symbiodinium life cycle involves both a sedentary coccoid phase and a dispersive motile phase (Freudenthal 1962; Fitt \& Trench 1983). When in symbiosis, the coccoid, non-motile symbionts are enclosed in membrane-bound vacuoles within the host's gastrodermal cells (Trench 1987), presumably isolating them from potential mates. Reproduction occurs exclusively through mitotic division of haploid, vegetative cells (Santos \& Coffroth 2003), a process that is tightly regulated by the host (Davy et al. 2012). When in the free-living phase, longitudinal and transverse flagella develop and cells closely resemble Gymnodinium spp. (Kawaguti 1944; Fitt \& Trench 1983; Trench \& Thinh 1995). Free-living Symbiodinium cells are motile, 
chemotactic (Fitt 1984; Pasternak et al. 2006) and phototactic (Hollingsworth et al. 2005), and viable cells are found in high abundance in the reef sediment and water column (Coffroth et al. 2006; Littman et al. 2008; Pochon et al. 2010; Takabayashi et al. 2011). Access to potential mates therefore appears to be unlimited ex hospite, presenting an ideal environment for sexual reproduction to occur (Trench 1997). Yet the conditions required to induce the sexual life-cycle in Symbiodinium continue to evade researchers, presenting a challenging obstacle to its thorough characterization.

Aside from providing the opportunity to eliminate deleterious mutations, recombination may lead to the acquisition of environmental adaptations, through the generation and proliferation of adaptive allele combinations under selection (Baillie et al. 1998; LaJeunesse 2001; Pettay et al. 2011). In the extreme case, recombination between divergent Symbiodinium lineages could facilitate the exchange of genetic material, vectored by a series of hybrid and backcross intermediates (introgression). Introgressive hybridization may provide the opportunity for a host-compatible symbiont lineage to rapidly acquire functional adaptations, supporting the evolution of the symbiosis. For these reasons, characterizing the sexual life-cycle and establishing the barriers of reproductive isolation in the Symbiodinium genus are important objectives in the effort to predict the ecological and evolutionary responses of corals in a changing climate.

\subsubsection{Hybridization in Symbiodinium?}

The crossing of individuals from genetically divergent lineages (hybridization; Arnold 1997) is evident in around $25 \%$ of plant species and $10 \%$ of animal species (Mallet 2005). Hybridization is thought to be common in the marine environment (Gardner 1997), though few studies have examined its occurrence in marine micro-eukaryotes (Casteleyn et al. 2009). While Symbiodinium does appear to reproduce sexually, it is not known whether members of different species, clades or subclades are sexually compatible or able to produce viable progeny. Analyses of polymorphic microsatellite loci have shown evidence of allelic recombination within, but not between Symbiodinium cp23S, ITS2 and $p s b A^{n c r}$ lineages (Santos et al. 2003b, 2004; Pettay et al. 2011; Thornhill et al. 2013a; LaJeunesse et al. 2014). In a phylogenetic analysis of clade $\mathrm{C}$ phylotypes on the GBR, organelle phylogenies were highly congruent with those of the nuclear rDNA, again indicating an absence of hybridization (Sampayo et al. 2009). Yet these studies represent only a small fraction of the 
worldwide Symbiodinium diversity, and hence more extensive sampling is needed to establish whether hybridization naturally occurs in this group. Furthermore, ITS sequence variants that produce additive patterns when visualized on electrophoretic gels (such as DGGE and SSCP) are often dismissed as co-dominant paralogs, without considering alternative explanations for their intra-genomic coexistence (or indeed determining whether the observed sequence variation is intra-genomic). For example, the Symbiodinium C1c ITS2 sequence was initially reported as a paralogous intra-genomic variant of $\mathrm{C} 1$, since the former was only found in association with the DGGE profile of the latter (LaJeunesse et al. 2003); however C1c was later assigned the alphanumeric status $\mathrm{C} 45$ when further sampling revealed its presence in isolation of C1 (LaJeunesse 2005). It is therefore possible that the additive pattern observed by LaJeunesse et al. (2003) could have resulted from hybridization between Symbiodinium $\mathrm{C} 1$ and $\mathrm{C} 45$.

Though yet to be identified in Symbiodinium, hybridization has been reported between members of divergent free-living dinoflagellate taxa (Edvardsen et al. 2003; Hart et al. 2007; Brosnahan et al. 2010). For example, Brosnahan et al. (2010) recovered hypnozygote cysts from Belfast Lough in Northern Ireland, and used nested single-cell qPCR (targeting the hyper-variable D1 and D2 regions of LSU rDNA) to demonstrate their hybrid origin. The progenitor taxa were identified as members of two ribosomal clades within the Alexandrium tamarense species complex (groups I and III). However, experimental crosses between these strains failed to yield viable progeny, with recombinant offspring undergoing no more than three cell divisions (Brosnahan et al. 2010). While post-zygotic mating barriers can cause hybrid lethality or sterility in crosses involving widely divergent taxa, more closely-related lineages can show comparatively weak reproductive isolation. For example, crosses of recently diverged, geographically isolated dinoflagellates within the Gymnodinium catenatum complex produced hypnozygotes with high excystment success and viable offspring (Blackburn et al. 2001). Successful hybridization is also apparent between the free-living dinoflagellates Dinophysis acuminata and D. norvegica, a species pair distinguished by eight nucleotide substitutions in the ITS1-5.8S-ITS2 region (Edvardsen et al. 2003). This level of divergence appears to be near the upper limit for reproductive compatibility in dinoflagellates, a threshold that roughly corresponds with other micro-eukaryote groups. In the freshwater diatom family Eunotiaceae, reproductive isolation and hybrid sterility generally evolve in mating pairs featuring ITS sequence divergence above $10 \%$ (Vanormelingen et al. 2008; Casteleyn et al. 2009). Barriers to reproductive isolation are also 
evident beyond $\sim 10 \%$ ITS divergence in the haplontic green algal family Volvocaceae (Coleman et al. 1994). Given that ecologically divergent Symbiodinium taxa can be differentiated by just a single base pair in the ITS1-5.8S-ITS2 region (0.2\% divergence; e.g. subclades $\mathrm{C} 1$ and $\mathrm{C} 3$ ), a thorough investigation of the occurrence and potential evolutionary effects of hybridization in the Symbiodinium genus is warranted.

\subsection{Inferring hybridization in Symbiodinium}

Establishing the incidence of hybridization and introgression has proven difficult in many natural populations. Identifying the genealogical origin of Symbiodinium individuals is further complicated by their unicellularity, haploidy, and the inability to establish many types in culture. Hybridization is often revealed using standard genotyping techniques such as multi-locus microsatellite analysis and amplified fragment length polymorphism (AFLP); however these PCR-based methods require a multi-genomic DNA template, limiting their utility to multicellular or cultivable single-cell organisms. The recent development of singlecell whole-genome amplification (WGA) methodology can provide the means to increase DNA template concentrations to sufficient levels for multi-locus genotyping, but these are currently expensive and technically demanding procedures (Handyside et al. 2004; Coskun \& Alsmadi 2007). Alternatively, a single locus may provide conditional evidence of hybridization provided that a suitable genetic region is targeted. Mitochondrial and chloroplast genomes are typically uniparentally inherited, and hence are rarely useful in identifying hybrids (Small et al. 2004). However, the intra-genomic co-dominance (additivity) of diagnostic nuclear alleles can provide convincing evidence that an individual is of hybrid origin (Soltis et al. 1992; Wendel et al. 1995; Sang et al. 1995; Brasier et al. 1999; Newcombe et al. 2000). Single-copy nuclear markers are preferable for such analyses, since they lack the NDPs often found in multi-copy gene families (Small et al. 2004). However, Symbiodinium is predominantly haploid (Santos \& Coffroth 2003; but see LaJeunesse et al. 2014); therefore recombination ultimately leads to the uniparental inheritance of single-copy alleles. The intra-genomic coexistence of polymorphic, biparentally inherited nuclear genes such as those of the rDNA cistron can provide conditional evidence of hybridization (Sang et al. 1995; Morrell \& Rieseberg 1998). In particular, the ITS2 has been extensively utilized in plant systematics, revealing numerous cases of hybridization, hybrid speciation and reticulate evolution (reviewed in Álvarez \& Wendel 2003). The multi copy nature of rDNA arrays also 
make their constituent sequences amenable to single-cell PCR amplification, circumventing the need to perform a WGA step or establish isoclonal cultures. However, a major limitation of using rDNA markers in hybridization analysis is that a similar additive genomic signature can also arise from the vestigial persistence of ancestral intra-genomic polymorphisms. If the most recent common ancestor of two putatively hybridizing taxa carried both diagnostic rDNA alleles within its genome, and insufficient time has passed since divergence for concerted evolution to fully homogenize the rDNA arrays in each lineage, an individual carrying both alleles may simply represent a member of one taxon that has retained copies of the allele diagnostic of the other (see Figure 1.5c). As such, distinguishing hybridization from incomplete concerted evolution requires the application of specialized statistical techniques (e.g. Bayesian methods that enable the inference of hybridization and introgression from multi-locus haplotypes; Mallet 2005), and should be reinforced with morphological, physiological and ecological data (Peterson et al. 2004; Vriesendorp \& Bakker 2005).

\subsection{Aims and objectives of this study}

This study aimed to establish whether hybridization occurs, or has occurred, between divergent Symbiodinium taxa. To address this question, a combination of genetic, physiological and ecological investigations were carried out on the reef-building coral Pocillopora damicornis, a widely distributed, vertically-transmitting species that hosts a diverse array of Symbiodinium taxa. Surveys and experiments were carried out at the highlatitude coral reef site of Lord Howe Island (LHI; Australia). The specific objectives were to:

1. Develop a single-cell PCR-DGGE protocol and a quantitative PCR (qPCR) assay to quantify ITS2 ratios within individual Symbiodinium cells, and hence screen for putative hybrids.

2. Develop a statistical framework to simultaneously test three competing hypotheses explaining the patterns of ITS2 additivity within P. damicornis symbionts: $\left(\mathrm{H}_{0}\right)$ colonies host a single clonal population of symbionts hosting a non-diagnostic polymorphism; $\left(\mathrm{H}_{1}\right)$ colonies host two populations of genetically distinct symbionts; and $\left(\mathrm{H}_{2}\right)$ three symbiont populations coexist within coral colonies, including two divergent taxa and an intermediate population of genetically heterogeneous Symbiodinium cells (putative hybrids). 
3. Develop a method to convert bulk-cell qPCR data (ITS2 sequence proportions) into the proportional abundance of genetically homogeneous and heterogeneous cells, and employ this method to model the spatiotemporal distribution of putative hybrids. The hypothesis tested in this objective was that homogeneous and heterogeneous symbionts undergo niche diversification, consistent with many natural hybridprogenitor systems.

4. Assess the physiological performance of heterogeneous symbionts relative to their homogeneous congeners. It was hypothesized that the different cell types are morphologically and physiologically distinct, and that each performs differently under various environmental conditions.

5. Establish whether physiological differences between genetically heterogeneous and homogeneous symbiont cells translate to differences in host fitness. The hypothesis tested was that corals hosting different proportions of homogeneous and heterogeneous symbionts show different growth and mortality rates in their natural environment.

The four subsequent data chapters address these objectives in the following format:

Chapter 2 addresses the first and second objectives. Individual symbionts were sampled from six $P$. damicornis colonies and analysed for intra-genomic ITS2 ratios. A finite mixture model was applied to test the three competing hypotheses outlined in objective 2 .

Chapter 3 addresses the third objective by reconciling ITS2 sequence proportions obtained from bulk-cell qPCR analysis with actual proportions of genetically homogeneous and heterogeneous cells. This facilitated a comprehensive analysis of the spatiotemporal distribution of each cell type within and between P. damicornis colonies on the LHI reef. Observed distribution patterns were correlated with a suite of environmental variables obtained from long-term monitoring.

Chapter 4 employs the methods developed in chapters 2 and 3 to test the hypothesis outlined in the fourth objective. The proportional abundance of genetically heterogeneous Symbiodinium cells was quantified in each of 15 P. damicornis colonies, and analyses of photosynthetic and respiratory oxygen flux were carried out at both ambient and elevated temperature. Analyses of symbiont morphology and physiology were also performed to further assess the candidacy of heterogeneous cells as putative hybrids. 
Chapter 5 involves the reciprocal transplantation of coral colonies between two distinct reef habitats. Corals were switched between a warm, well lit inner-lagoon site and a reef margin location characterised by low light levels, strong wave action and high macroalgal growth. The growth and survival of corals were assessed in relation to the proportional abundance of genetically heterogeneous Symbiodinium cells within their symbiont consortia.

Chapter 6 provides a review of the combined evidence for hybridization between Symbiodinium phylotypes, and speculates on the possible evolutionary implications thereof. Future directions are suggested for the unambiguous confirmation of hybridization in symbiotic dinoflagellates, and the establishment of its location, frequency, and consequences for coral fitness. The possible application of controlled hybridization in the 'evolutionary rescue' of corals in a deteriorating environment is also discussed. 


\section{Chapter 2: Measuring intra-genomic variation in Symbiodinium}

\subsection{Introduction}

Until the mid- $20^{\text {th }}$ century, hybridization was thought to occur rarely and have little evolutionary significance (Mayr 1963). However, a major paradigm shift extolled the incidence and importance of hybridization, led principally by the pioneering work of American botanist Edgar S. Anderson (Anderson 1949; Anderson \& Stebbins 1954). This was articulated by his colleague Warren $\mathrm{H}$. Wagner who recalled "We used to make fun of Edgar Anderson by saying that he was finding hybrids under every bush - then we realized that even the bushes were hybrids" (Abbott et al. 2013). The so-called 'molecular revolution' has increased our awareness of natural hybridization further still, with current estimates of around $25 \%$ of plant species and $10 \%$ of animal species undergoing hybridization (Mallet 2005). Yet the phylogenetic and statistical criteria that constitute convincing evidence of natural hybridization and introgression remain the subject of contention, and may depend on the life-history characteristics of the taxa in question (Vriesendorp \& Bakker 2005).

Morphological similarities among symbiotic dinoflagellates highlight the need to use appropriate genetic tools when establishing the incidence of hybridization in this group. The internal transcribed spacer 2 (ITS2) region of nuclear ribosomal DNA (rDNA) is the most well-characterised and commonly-used marker in Symbiodinium systematics, primarily due to its high taxonomic resolution (van Oppen \& Gates 2006; Stern et al. 2012). Unlike alternative mitochondrial- and chloroplast-encoded sequences and single-copy nuclear genes, the ITS2 is bi-parentally inherited in most sexually-reproducing organisms, and hence the intra-genomic coexistence of divergent sequence variants can reveal the occurrence of ancient and recent hybridization events (Baldwin et al. 1995; Álvarez \& Wendel 2003). However, the multiplecopy nature of the rDNA cistron renders it subject to intra-genomic variation from a variety of other processes, including the generation of paralogous somatic mutations and the degeneration of functional genes into pseudo-genes (Thornhill et al. 2007). Establishing whether a given ribotype is taxonomically meaningful requires the analysis of individuals rather than multi-genomic samples, necessitating a single-cell approach for unicellular organisms such as Symbiodinium (Correa \& Baker 2009; Miranda et al. 2012). The generation of isoclonal cell-lines from individual Symbiodinium cells offers a partial solution 
(Thornhill et al. 2007); however frequent somatic mutations may lead to cultures that are not representative of the original cell (van Oppen et al. 2011). Single-cell PCR (scPCR) provides an obvious advantage in distinguishing between intra and inter-genomic sequence variation (Tengs et al. 2000; Edvardsen et al. 2003); yet this method has not been widely used in Symbiodinium systematics. This is primarily due to its time-consuming nature, and the difficult task of disrupting the recalcitrant cell wall to extract the nucleic acids. A lack of suitable methodology for isolating, extracting and sequencing DNA from individual Symbiodinium cells has meant that intra-genomic variation in this genus has remained virtually unexplored (Stat et al. 2012).

While establishing the dominant ribotype within individual cells is necessary to establish diversity estimates (Correa \& Baker 2009), it is also beneficial to quantify intra-genomic sequence variation when assessing the incidence of hybridization. This is because $\mathrm{F}_{1}$ hybrids are expected to inherit relatively similar rDNA copy numbers from each parent (Baldwin et al. 1995; Brosnahan et al. 2010), and polymorphism ratios can indicate the direction of introgression and/or concerted evolution (Wendel et al. 1995; De Castro et al. 2013). Fluorogenic-probe based qPCR analysis now offers sufficient sensitivity to quantify ITS2 variants at the subclade level, and when preceded by a PCR pre-amplification step (nested qPCR; e.g. Brosnahan et al. 2010), it can be used to quantify polymorphic ribotypes within the individual dinoflagellate genome. However, while the intra-genomic co-dominance (additivity) of diagnostic ITS2 variants can allude to the occurrence of hybridization and even introgression, a similar genomic signature can also rise from the incomplete concerted evolution of ancestral polymorphisms (ICEAP). For this reason, a statistical framework is needed to distinguish between these conflicting origins of intra-genomic rDNA additivity.

In this chapter, I attempt to address these methodological limitations by developing: (1) a single-cell isolation and DNA extraction protocol for Symbiodinium; (2) a single-cell PCRDGGE method to screen for Symbiodinium individuals with additive ITS2 repeats; (3) a nested PCR-qPCR assay to quantify intra-genomic ITS2 sequence polymorphisms within individual cells; and (4) a statistical framework to identify admixture in Symbiodinium populations based on proportions of ITS 2 sequence variants within the genome. The model selection criterion developed in (4) was then employed to test whether the P. damicornis symbiont consortium consists of $\left(\mathrm{H}_{0}\right)$ a single clonal population of symbionts featuring a nondiagnostic polymorphism (NDP); $\left(\mathrm{H}_{1}\right)$ two populations of divergent, homogeneous symbionts; or $\left(\mathrm{H}_{2}\right)$ a mixture of genetically homogeneous symbionts and heterogeneous cells, 
representing putative hybrids (Figure 2.1a). The proportional abundance of ITS2 polymorphisms within each Symbiodinium cell was also assessed to explore the potential for gene flow via introgressive hybridization (Figure 2.1b).

(a)

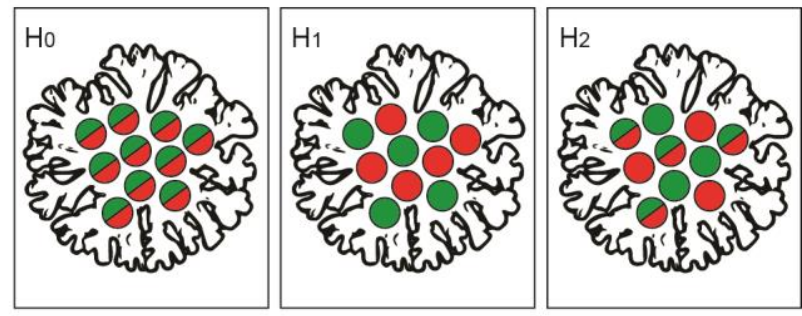

(b)

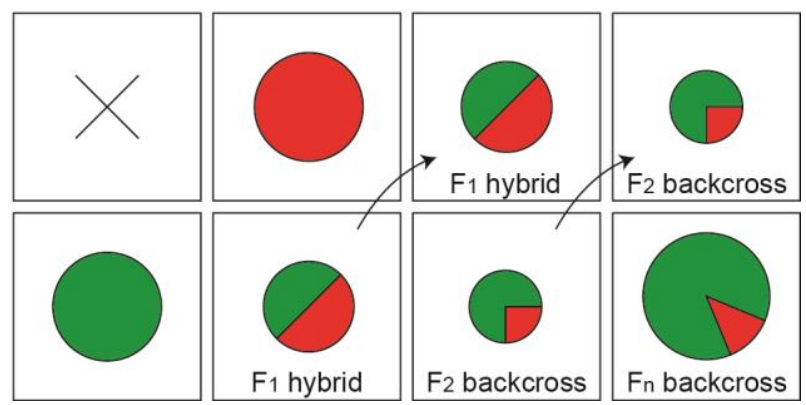

Figure 2.1 Conflicting origins of intragenomic variation in Symbiodinium

Red and green colorations represent divergent ITS2 sequences, with monocoloured cells featuring homogeneous or near-homogeneous ITS2 arrays and bicoloured cells hosting polymorphic ribotypes. Schematics show (a) competing hypotheses of sequence homology within $P$. damicornis-associated Symbiodinium, with a single clonal population hosting a non-diagnostic polymorphism (NDP) under $\mathrm{H}_{0}$, exclusively homogeneous symbionts under $\mathrm{H}_{1}$ and hybridization between individuals from genetically distinct populations under $\mathrm{H}_{2}$; and (b) introgressive hybridization, with differential fitness between hybrid classes (shown in a size gradient). Many backcross generations ( $n$ ) may occur before an increase in fitness is realized. Genetic isolation occurs when one or more hybrid/backcross classes suffer from insurmountably low mean fitness.

\subsection{Methods}

\subsubsection{Study species and location}

This study was carried out at the world's southernmost coral reef at Lord Howe Island (LHI; Australia). This isolated $14.5 \mathrm{~km}^{2}$ volcanic remnant is located around $600 \mathrm{~km}$ east of the Australian mainland, and some 200km to the south of the Elizabeth and Middleton Reefs Marine National Park Reserve. The LHI reef hosts at least 83 species of scleractinian coral, (many of which are endemic; Harriott et al. 1995), and a correspondingly diverse and endemic Symbiodinium assemblage (Wicks et al. 2010). Reef fauna are regularly exposed to widely variable thermal conditions (Harriott \& Banks 2002), and cold-stress is known to play a defining role in determining species boundaries at this site (Veron \& Done 1979). In certain 
reef-margin areas, a stark transition from coral- to macro-algal dominated benthic assemblages is apparent (Allen \& Paxton 1974).

The host species investigated in this study was the widely-distributed coral Pocillopora damicornis, a thermally-sensitive but fast-growing species that forms a dominant component of many Indo-Pacific reefs (including that of LHI; Veron 2000). Colonies at LHI can grow to several metres in diameter in sheltered inner-lagoon habitats, and form a small compact clump-like morphology in exposed locations (Miller \& Ayre 2004). P. damicornis is hermaphroditic and shows an unusual dual-reproductive mode, with the majority of offspring consisting of brooded asexual larvae, complimented by the cryptic simultaneous broadcastspawning of sexual gametes in some areas (Combosch \& Vollmer 2013). This species shows a predominantly sexual reproductive mode at LHI, where it occasionally undergoes intergeneric hybridization with Stylophora pistillata (Miller \& Ayre 2004). This may arise from suboptimal abiotic conditions selecting for 'extreme' hybrid phenotypes, and/or a low availability of conspecific gametes (Willis et al. 2006). P. damicornis transmits symbionts vertically from parent to offspring, and can form a symbiotic relationship with a large array of genetically and physiologically distinct Symbiodinium taxa. In Australian waters alone, $P$. damicornis is found in association with $S$. goreauii, S. glynni, S. trenchii, and numerous other undescribed ITS2 types including C1b, C1c, C1c-ff, C1h, C1j, C33, C33a, C42, C42a, C42b, C100, C103, C118, C125 and C126 (LaJeunesse et al. 2003, 2004; Sampayo et al. 2007; Ulstrup et al. 2008; Stat et al. 2008a; Wicks et al. 2010; Silverstein et al. 2011; Tonk et al. 2013). Of these, P. damicornis colonies have been reported hosting Symbiodinium C100, C103 and C118 at LHI (Wicks et al. 2010).

\subsubsection{Sample collection and DNA isolation}

Coral sampling was carried out in March 2012 at North Bay and Ned's Beach, Lord Howe Island, Australia. Three Pocillopora damicornis colonies were sampled from each site by divers using snorkel (North Bay; depth 1-3 m) or SCUBA (Ned's Beach; depth 14-16m). In all cases, colonies were sampled at least $2 \mathrm{~m}$ from each other to reduce the risk of selecting ramets. Three small branch tips $\left(\sim 1 \mathrm{~cm}^{3}\right)$ were taken from each colony using diagonal pliers, and preserved in DMSO preservation buffer (20\% DMSO, $250 \mathrm{mM}$ EDTA, NaCl saturated, $\mathrm{pH}$ 8.0; Seutin et al. 1991). Coral samples were stored at $-20{ }^{\circ} \mathrm{C}$ prior to DNA analysis. A $0.12 \mathrm{~cm}^{2}$ area of tissue was removed from the skeleton in $1.5 \mathrm{ml}$ of $0.22 \mu \mathrm{m}$ filtered seawater 
(FSW), delivered at high velocity through a circular stencil. A $10 \mu \mathrm{l}$ sub-sample was taken and centrifuged at $16,100 \times \mathrm{g}$ for 5 min to pellet the Symbiodinium fraction. The supernatant was discarded and the pellet re-suspended in $100 \mu \mathrm{l}$ DNA buffer (DNAB; 0.4M NaCl, 50mM EDTA, $\mathrm{pH}$ 8.0). Individual cells ( $n=30$ from each colony) were hand-picked under a light microscope using a heat-elongated glass micro-pipette. Each cell was washed three times in 2 $\mu \mathrm{DNAB}$, transferred to a $1.7 \mathrm{ml}$ micro-centrifuge tube with $50 \mathrm{mg}$ acid-washed glass beads (710-1180 $\mu \mathrm{m}$; Sigma-Aldrich), and milled for $1 \mathrm{~min}$ at $50 \mathrm{~Hz}$ (Qiagen TissueLyser LT; Qiagen) to disrupt the cell-wall and release the nucleic acids. TE buffer (10 mM Tris- $\mathrm{HCl}$; 1 mM EDTA; $\mathrm{pH}=8.0$ ) was then added to a final volume of $20 \mu$. For each colony, the extraction process was carried out with the symbiont cell omitted (but with coral tissue homogenate included), to ensure that only intracellular DNA contributed to the PCR amplification signal.

\subsubsection{End-point PCR, DGGE and DNA sequencing}

Single-cell DNA template solutions generally contained insufficient DNA for direct PCRDGGE and qPCR analysis. The partial $n r 5.8 S$, ITS2 and partial $n r 28 S$ regions were therefore pre-amplified using a shortened end-point PCR protocol, with the outer primers ITSintfor2 (LaJeunesse 2002) and ITS2Rev2 (Stat et al. 2009). Thermal cycling included an initial denaturation step of $3 \mathrm{~min}$ at $95^{\circ} \mathrm{C}$ followed by 24 cycles of 15 seconds at $95{ }^{\circ} \mathrm{C}, 15$ seconds at $56{ }^{\circ} \mathrm{C}$ and 10 seconds at $72{ }^{\circ} \mathrm{C}$ (carried out on an Applied Biosystems Veriti thermocycler). Each reaction contained $10 \mu \mathrm{l}$ of DNA template solution, 1x MyTaq PCR reaction mix (Bioline), $15 \mathrm{pmol}$ each primer, and deionised sterile water to a total volume of $25 \mu$. Multiple pre-amplification reactions on individual template solutions were prevented by the limited amount of target DNA available. A template-free control reaction was included with each run.

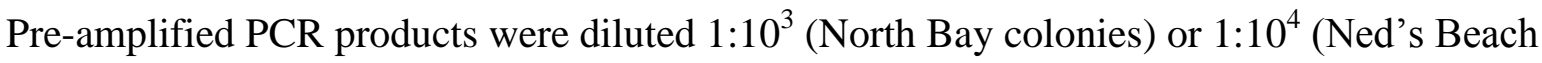
colonies) in deionised sterile water prior to PCR-DGGE and qPCR analysis. These differences were due to shortages of DNA template solutions from the Ned's Beach colonies, which were used for the initial assay development and optimization process. PCR amplification for DGGE was carried out using the primers ITSintfor2 and ITS2CLAMP (LaJeunesse 2002). Cycling conditions were as described above, except an additional 16 thermal cycles were run (40 in total). PCR products $(20 \mu \mathrm{l})$ were loaded on $200 \times 200 \times 0.75$ 
$\mathrm{mm}, 8 \%$ denaturing polyacrylamide gels (25-50\% denaturant gradient), and run in $1 \mathrm{x} \mathrm{TAE}$ at $150 \mathrm{~V}$ for $7 \mathrm{~h}$ at $60{ }^{\circ} \mathrm{C}$ (DCode system; BioRad) alongside known ITS2 sequences of Symbiodinium C100 and C109. Following electrophoresis, gels were stained with ethidium bromide and viewed on a UV trans-illuminator (FirstLight UVP). Five representative (dominant) bands at each position were excised, milled for 1 minute at $50 \mathrm{~Hz}$ with $50 \mathrm{mg}$ glass beads and $200 \mu \mathrm{TE}$ buffer, and re-amplified with both clamped and non-clamped primers (LaJeunesse 2002). DGGE was carried out on clamped PCR products to ensure a single band migrated to the identical position from where it was excised. Corresponding nonclamped products were cleaned with ExoSAP-IT (USB), and sequenced by the Macrogen

Sequencing Service (Macrogen Inc., Seoul, South Korea). Sequences were manually checked and aligned in Geneious v 7.0 (Biomatters) and a BLAST search was carried out against Symbiodinium ITS2 sequences available in Genbank. Novel sequences were assigned alphanumeric ITS2 nomenclature (c.f. LaJeunesse 2001, 2002) and deposited into the Genbank database. The un-rooted statistical parsimony network of Symbiodinium ITS2 phylotypes found within Pocilloporid corals at LHI (Wicks et al. 2010) was updated in TCS v 1.21 (95\% connection limit; gaps assigned fifth character state; Clement et al. 2000).

\subsection{4. $q P C R$ analysis of Symbiodinium ITS2 ratios}

For qPCR analysis, the universal primers CInnerFor (5'TGGCTTGTTAATTGCTTGGTTCT-3') and CInnerRev (5'-ACCTGCATCCCAGCGGTT$\left.3^{\prime}\right)$ were developed, in addition to the custom TaqMan fluorogenic probes $\mathrm{C} 100^{+}$and $\mathrm{C} 100^{-}$ (5'-TTTTACTTGAGTGACACCGC-3' and 5'-CTTTACTTGAGTGACGCTGC-3', respectively; Life Technologies). The probe $\mathrm{C} 100^{+}$was designed to quantify the number of ITS2 sequences of type $\mathrm{C} 100$ in a given sample (denoted $\mathrm{C}_{\mathrm{C} 100}$ ), while the $\mathrm{C} 100^{-}$probe was developed to quantify the copy-number of all clade C ITS 2 sequences other than type C100. All primers and probes were initially checked for specificity by conducting a BLAST search against sequences deposited in Genbank (Altschul et al. 1990). To obtain purified DNA sequences for qPCR calibration, PCR products (types C100, C103, C109 and C118 extracted from P. damicornis, and C3 obtained from the VUW Symbiodinium laboratory culture collection) were cloned using the TOPO TA kit (Invitrogen Life Technologies). Plasmid colonies were incubated overnight on selective LB agar plates containing ampicillin, IPTG and XGAL (Bioline). DNA was extracted from positive transformants, purified using a 
plasmid Mini-Prep kit (Invitrogen Life Technologies), and sequenced as above with the M13 primer set. Plasmid DNA template concentrations were estimated using a Pearl Nanophotometer (Implen), diluted to approximately $10^{-3} \mathrm{ng} \mathrm{l}^{-1}$, and five $\log _{10}$ serial dilutions were constructed to generate standard curves and test the accuracy and precision of the assay. All qPCR reactions were carried out in triplicate (standard curves) or duplicate (template solutions) on an Applied Biosystems StepOne instrument (Life Technologies), alongside a template-free control reaction. Each TaqMan qPCR reaction contained $4 \mu \mathrm{l}$ template, 1x TaqMan Universal Mastermix II (Life Technologies), 1x TaqMan fluorogenic probe (Life Technologies), $18 \mathrm{pmol}$ each primer, and deionised sterile water to a total volume of $20 \mu \mathrm{l}$. Thermal cycling conditions involved an initial $10 \mathrm{~min}, 95{ }^{\circ} \mathrm{C}$ denaturation step followed by 40 cycles of 15 seconds at $95{ }^{\circ} \mathrm{C}$ and $1 \mathrm{~min}$ at $60{ }^{\circ} \mathrm{C}$. Cycle threshold $\left(\mathrm{C}_{\mathrm{t}}\right)$ values were determined as the cycle at which the change in fluorescence was significantly different to the background level $\left(\Delta R_{n}=0.05\right.$; obtained using the instrument's built-in algorithm). $C_{t}$ values below the standard curve intercept (see Appendix $B$ ) and featuring sufficiently low standard deviations $(<0.5)$ were included in the analysis.

To ensure that the TaqMan assays $\mathrm{C} 100^{+}$and $\mathrm{C} 100^{-}$detected all Symbiodinium clade C sequences present within each sample, the total ITS2 copy number (denoted $\mathrm{C}_{\text {TOTAL }}$ ) in each Symbiodinium cell from the North Bay colonies was also estimated using SYBR qPCR analysis. Reactions were carried out as above, except Power SYBR Green Mastermix (Life Technologies) was used in place of TaqMan Universal Mastermix II, fluorogenic probes were omitted, and $C_{t}$ values were generated using the $\Delta R_{n}$ threshold value of 0.3 . A melt curve (temperature elevation from $60{ }^{\circ} \mathrm{C}$ to $95^{\circ} \mathrm{C}$ in $0.3{ }^{\circ} \mathrm{C}$ increments each of $15 \mathrm{~s}$ duration) was included at the end of each run to ensure only detectable target sequences were amplified. Template solutions yielding $\mathrm{C}_{\mathrm{t}}$ values below the standard curve intercept and melting temperatures $\left(\mathrm{T}_{\mathrm{m}}\right)$ within $1{ }^{\circ} \mathrm{C}$ of plasmid $\mathrm{T}_{\mathrm{m}}$ values were included in the analysis. The ITS2 copy number within each cell ( $\mathrm{C}_{\mathrm{TOTAL}}$; as determined from SYBR qPCR analysis) was compared to the sum of those given by the $\mathrm{C} 100^{+}$and $\mathrm{C} 100^{-}$TaqMan assays using linear regression (parameters constrained; intercept $=0$, slope $=1$ ). Finally, a mixture test was carried out to assess the ability of the TaqMan qPCR assay to accurately predict the proportion of total Symbiodinium clade C ITS2 copies that were of type $\mathrm{C} 100\left(\mathrm{C}_{\mathrm{C} 100}: \mathrm{C}_{\text {TOTAL }}\right.$ ratio). Eight mixtures were constructed from plasmid C100 and C109 DNA template solutions (diluted to approximately 200 ITS2 copies $\mu 1^{-1} ; \mathrm{C}_{\mathrm{C} 100}: \mathrm{C}_{\text {TOTAL }}$ ratios $=0,0.02,0.10$, 0.4, 0.6, 0.9, 0.98 and 1; see Appendix $B$ for $\mathrm{C}_{\mathrm{t}}$ values) and $\mathrm{qPCR}$ reactions were carried out 
in duplicate as above. The ability of the combined TaqMan assay to predict $\mathrm{C}_{\mathrm{TOTAL}}$ and $\mathrm{C}_{\mathrm{C} 100}: \mathrm{C}_{\mathrm{TOTAL}}$ was assessed using linear regression (parameters constrained; intercept $=0$, slope $=1)$.

\subsubsection{Statistical analysis}

To assess the relationship between the total ITS2 copy number and the proportion of copies that were of type $\mathrm{C} 100$, a non-linear regression curve (second order polynomial) was fitted to the bivariate $\mathrm{C}_{\mathrm{C} 100}: \mathrm{C}_{\text {TOTAL }}$ versus $\mathrm{C}_{\text {TOTAL }}$ data in Sigmaplot v11.0 (Systat). Values of $\mathrm{C}_{\mathrm{C} 100}: \mathrm{C}_{\mathrm{TOTAL}}$ were arcsin transformed and compared between colonies (Colony) and between branches within colonies (Branch(Colony)) using nested ANOVA (Im function in R; R Development Core Team 2011). Three competing hypotheses were evaluated to explain the ITS2 sequence variation within and between the symbionts of $P$. damicornis: $\left(\mathrm{H}_{0}\right)$ colonies host a single population of genetically heterogeneous symbionts, versus $\left(\mathrm{H}_{1}\right)$ colonies host two populations of genetically distinct, homogeneous symbionts, versus $\left(\mathrm{H}_{2}\right)$ colonies host distinct populations of genetically homogeneous and heterogeneous symbionts, consistent with the occurrence of hybridization (Figure 2.1) The frequency distribution of $\mathrm{C}_{\mathrm{C} 100}$ : $\mathrm{C}_{\mathrm{TOTAL}}$ within a coral colony $(\mathrm{X})$ is expressed in model form as:

$\mathrm{H}_{0}: \mathrm{X} \sim \operatorname{Beta}(\alpha, \beta), \alpha>1, \beta>1$

Equation 2.1

$\mathrm{H}_{1}: \mathrm{X} \sim \operatorname{Beta}(\alpha, \beta), \alpha<1, \beta<1$ Equation 2.2

$\mathrm{H}_{2}: \mathrm{X} \sim \pi \operatorname{Beta}\left(\alpha_{1}, \beta_{1}\right)+(1-\pi) \operatorname{Beta}\left(\alpha_{2}, \beta_{2}\right), 0<\pi<1$

Equation 2.3

where $\alpha$ and $\beta$ are the shape parameters of the beta function, and $\pi$ denotes the proportion of symbionts belonging to each component of the mixture model. Mixed beta functions were fitted to the $\mathrm{C}_{\mathrm{C} 100}: \mathrm{C}_{\mathrm{TOTAL}}$ frequency distributions of each coral colony, and maximum likelihood parameter values were solved using the optim function in R (L-BFGS-B method; R Development Core Team 2011). A range of starting parameter values were used at each optimization stage to ensure that a universal log-likelihood maximum was reached. Hypothesis evaluation was based on weighted AICc values $\left(w_{i}\right)$, with those above 0.90 considered to provide unambiguous support for a candidate model (Burnham \& Anderson 2002). 


\subsection{Results}

\subsubsection{DGGE and DNA sequencing}

The excision and sequencing of DGGE bands revealed that all six $P$. damicornis colonies hosted Symbiodinium ITS2 types C100 (Genbank accession number HM222433; Wicks et al. 2010) and C109 (Genbank accession number KJ530690; novel sequence). Although two divergent ITS2 sequences were retrieved, three distinct DGGE band profiles were observed among the 180 individual cells analysed. These corresponded to Symbiodinium cells featuring a homogeneous C100 array, those featuring a homogeneous C109 array, and those with a heterogeneous mixture of both ITS2 types (Figure 2.2). Four of the six colonies analysed hosted a consortium of Symbiodinium cells that included all three profiles (two colonies from each site), while the remaining two colonies hosted only homogeneous C100 symbionts and those producing the heterogeneous band-pattern (Figure 2.2a). No amplification signal was detected from template-free controls or the extractions with symbiont cells omitted, indicating an absence of extracellular DNA contamination. 
(a)

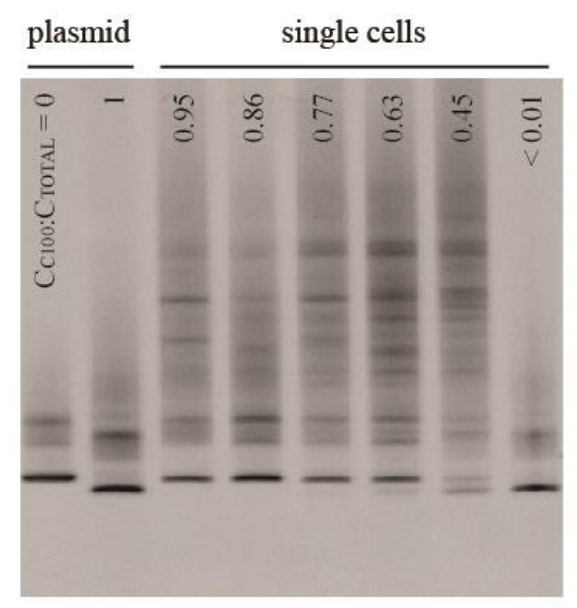

(b)

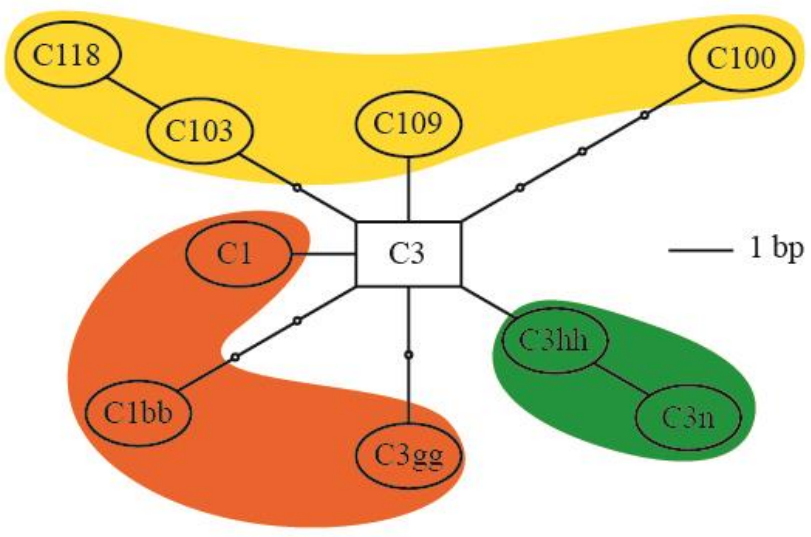

Figure 2.2 Sequence variation among Pocilloporid-associated Symbiodinium at LHI ITS2 sequence variation between and within the Symbiodinium genome is shown by: (a) DGGE profiles of individual symbionts from $P$. damicornis colonies at Lord Howe Island, featuring a range of $\mathrm{C}_{\mathrm{C} 100}: \mathrm{C}_{\mathrm{TOTAL}}$ ratios (alongside plasmid-purified C100 and C109 DNA); and (b) an un-rooted statistical parsimony network showing the phylogenetic relationships between derived Pocilloporidassociated Symbiodinium types found at Lord Howe Island (ellipses) and the ancestral C3 root (rectangle; modified from Wicks et al. 2010). Small circles in (b) represent hypothetical intermediate sequences, each distinguished from its neighbor by a single nucleotide substitution or gap. $P$. damicornis-associated types are shown in yellow, while those found in association with Stylophora pistillata and Seriatopora hystrix are shown in orange and green, respectively. See Appendix A for Genbank accession numbers.

\subsection{2. $q P C R$ estimation of intra-genomic ITS2 ratios}

The universal primers CInnerFor and CInnerRev were identically matched to conserved regions within the ITS2 of Symbiodinium C100 and C109. These primers also share identical sequences or single-base pair mismatches with nearly all clade $\mathrm{C}$ sequences currently available in the Genbank database, including those found within the corals of LHI (Wicks et al. 2010). A sequence BLAST analysis of the target probe $\mathrm{C}_{100^{+}}$revealed a high specificity for Symbiodinium C100, with at least two nucleotide substitutions differentiating it from the majority of other clade $\mathrm{C}$ sequences in Genbank (positioned 16 and 18 base pairs from the 5' end of the probe). The cytosine at the $5^{\prime}$ end of the probe $\mathrm{C}^{100^{-}}$is mismatched to $\mathrm{C} 100, \mathrm{C} 109$ and the majority of other clade C Symbiodinium types (including the ancestral types $\mathrm{C} 1$ and C3). This mismatch had no effect on the reaction efficiency when tested on ITS2 types C109 and C103 (95\% < E < 100\%; see Appendix B); however it served to prevent cross- 
hybridization with the $\mathrm{C} 100$ sequence. With this exception, $\mathrm{C}^{-100^{-}}$shared an identical sequence to most clade C Symbiodinium types available in Genbank, including the ancestral types C1, C3 and all derived types previously found in association with P. damicornis at LHI (Wicks et al. 2010). Standard curve analysis of both TaqMan assays revealed acceptable reaction efficiencies when matched to their respective target sequences $\left(\mathrm{C} 100^{+}\right.$to $\mathrm{C} 100$; $\mathrm{C} 100^{-}$to both $\mathrm{C} 109$ and $\mathrm{C} 103 ; 95 \%<\mathrm{E}<100 \%$ and $\mathrm{R}^{2}>0.99$ in all cases; see Appendix B). qPCR analysis of known plasmid DNA mixtures yielded high accuracy and precision in estimating $\mathrm{C}_{\mathrm{C} 100}: \mathrm{C}_{\text {TOTAL }}$ (constrained linear regression; $\mathrm{R}^{2}=0.998 ;$ Appendix $B$ ) and an absence of cross-hybridization. TaqMan qPCR-generated $\mathrm{C}_{\text {TOTAL }}$ values within each Symbiodinium cell were highly correlated with, and not significantly different from those obtained from the SYBR qPCR assay (constrained linear regression; $\mathrm{R}^{2}=0.978$; see Appendix B), indicating a negligible incidence of clade C ITS2 types other than those detected by $\mathrm{C} 100^{+}$and $\mathrm{C} 100^{-}$. SYBR qPCR melt curve analysis showed no $\mathrm{T}_{\mathrm{m}}$ differences between plasmid $\mathrm{C} 100$ and $\mathrm{C} 109$, and all single-cell templates yielded single $\mathrm{T}_{\mathrm{m}}$ peaks within $1{ }^{\circ} \mathrm{C}$ of the plasmid-generated values.

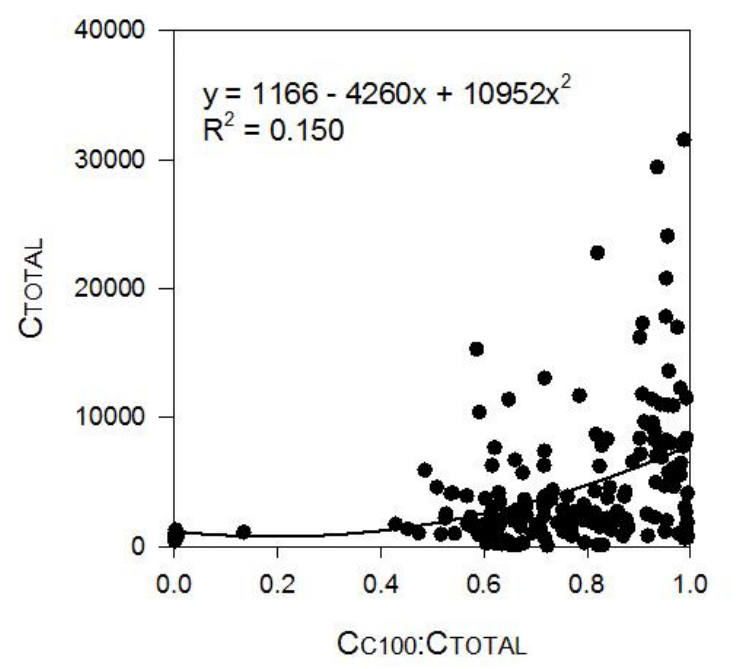

\section{Figure 2.3 Variation in ITS2 copy numbers within Symbiodinium cells} A non-linear relationship existed between the proportion of ITS2 copies of type $\mathrm{C} 100$ $\left(\mathrm{C}_{\mathrm{C} 100}: \mathrm{C}_{\mathrm{TOTAL}}\right)$ and the total number of ITS2 copies within the cell $\left(\mathrm{C}_{\text {TOTAL }}\right)$. Homogeneous C100 cells hosted significantly more ITS2 copies than the genetically heterogeneous cells, or those featuring a homogeneous C109 array (second order polynomial regression, $\mathrm{p}=$ 0.027).

Within-cell ITS 2 copy numbers $\left(\mathrm{C}_{\mathrm{TOTAL}}\right)$ were highly variable, ranging from less than 500 to over 30,000. C100 was the dominant ITS2 type in the majority of cells analysed, with $\mathrm{C}_{\mathrm{C} 100}$ : $\mathrm{C}_{\text {TOTAL }}$ ratios ranging between 0 and 0.987 (Figure 2.3; see Appendix $B$ for $\mathrm{C}_{\mathrm{t}}$ values). The remaining ITS2 copies appeared to be primarily of type C109, since this was the only other sequence detected in the DGGE analysis. DGGE band intensities generally reflected 
qPCR-generated $\mathrm{C}_{\text {TOTAL }}$ values, and in cases where both C100- and C109-diagnostic bands were present, their relative intensity gave a qualitative indication of $\mathrm{C}_{\mathrm{C} 100}: \mathrm{C}_{\mathrm{TOTAL}}$. However, the $\mathrm{C} 109$ band was generally very faint in cells featuring $\mathrm{C}_{\mathrm{C} 100}: \mathrm{C}_{\mathrm{TOTAL}}$ ratios greater than 0.75, and universally undetectable in those above 0.85 (Figure 2.2a; see Appendix B). A significant nonlinear correlation between $\mathrm{C}_{\mathrm{C} 100}$ : $\mathrm{C}_{\mathrm{TOTAL}}$ and $\mathrm{C}_{\mathrm{TOTAL}}$ revealed that ITS2 copy numbers were higher on average in genetically homogeneous $\mathrm{C} 100$ cells than in either the heterogeneous C100/C109 cells or the homogeneous C109 cells (non-linear regression, $\mathrm{p}<$ 0.027; $\mathrm{R}^{2}=0.15$; Figure 2.3). Within-cell $\mathrm{C}_{\mathrm{C} 100}: \mathrm{C}_{\mathrm{TOTAL}}$ ratios did not differ between branches within colonies, but varied between colonies (nested ANOVA, $p=0.82$ and $<0.01$ for Branch(Colony) and Colony effects, respectively; Table 2.1).

Table 2.1 Nested ANOVA for intra-genomic variation in ITS2 ratios

\begin{tabular}{llllll}
\hline Source & df & SS & MS & $\boldsymbol{F}$ & $\boldsymbol{P}$ \\
\hline Between colonies & 5 & 4.07 & 0.81 & 24.23 & 0.001 \\
Between branches within colonies & 12 & 0.40 & 0.03 & 0.61 & 0.82 \\
Error & 162 & 8.87 & 0.05 & & \\
\hline
\end{tabular}

The model design used in the nested ANOVA analysis was $\mathrm{C}_{\mathrm{C} 100}: \mathrm{C}_{\mathrm{TOTAL}} \sim$ Colony $\mathrm{x}$ Branch (Colony). $\mathrm{C}_{\mathrm{C} 100}: \mathrm{C}_{\text {TOTAL }}$ ratios were arcsin transformed prior to analysis. Branches within colonies were pooled for subsequent mixture model fitting.

The application and evaluation of competing beta models based on $\mathrm{C}_{\mathrm{C} 100}$ : $\mathrm{C}_{\text {TOTAL }}$ ratios revealed the presence of multiple symbiont clusters in all six colonies. In all cases, the twocomponent beta mixture model representative of $\mathrm{H}_{2}$ provided the best fit of the candidate models ( $w_{i}>0.90$ for all colonies; Table 2.2). Three modes were present in colonies a, b, $\mathrm{d}$ and e, representing clusters of genetically homogeneous C100 cells, homogeneous C109 cells, and heterogeneous C100/C109 cells. Two modes were detected in colonies c and f, representing coexisting populations of homogeneous C100 cells and heterogeneous C100/C109 cells (Figure 2.4). The proportion of genetically heterogeneous symbiont cells in the consortium ranged from $7 \%$ in colony c to $88.5 \%$ in colony a. 

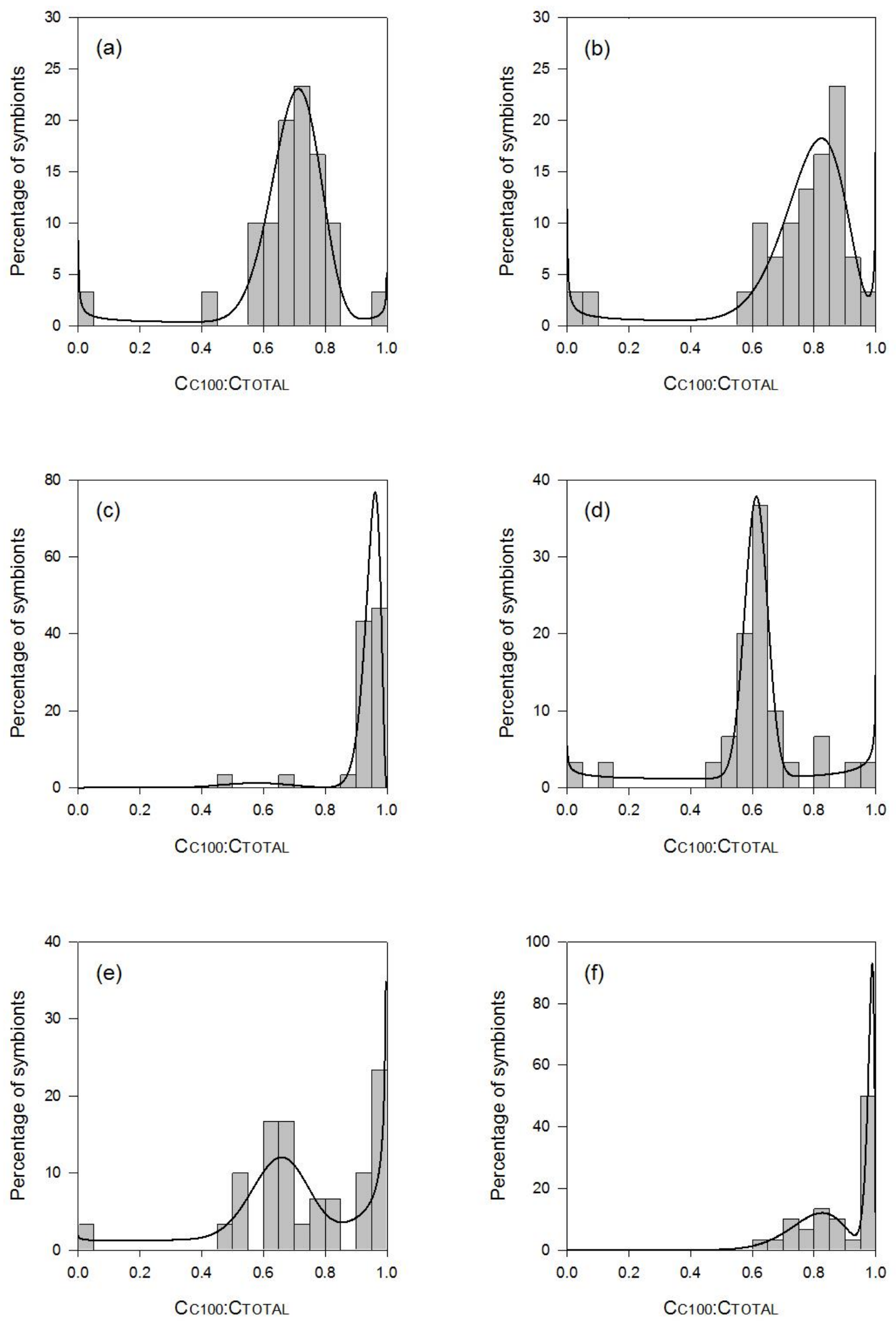
Figure 2.4 (previous page) Frequency distributions of intra-genomic ITS2 ratios

Colonies a-c were sampled from North Bay (1-3m), and colonies d-f from Ned's Beach (14-18m). Vertical bars represent the percentage of symbiont cells within each $\mathrm{C}_{\mathrm{C} 100}: \mathrm{C}_{\text {TOTAL }}$ category $(n=30$ cells for each colony). Overlying probability density functions are optimized two-component beta mixtures (see Table 2.2 for parameter estimates).

Table 2.2 Summary of optimized beta mixture models

\begin{tabular}{|c|c|c|c|c|c|}
\hline $\begin{array}{l}\text { Colony } \\
\text { ID }\end{array}$ & Site & $\begin{array}{l}\text { Best-fit } \\
\text { hypothesis }\end{array}$ & $\begin{array}{l}\text { Model } \\
\text { Equation }\end{array}$ & $\begin{array}{l}\text { Proportion } \\
\text { heterogeneous }\end{array}$ & $w_{i}$ \\
\hline $\mathbf{a}$ & $\begin{array}{l}\text { North } \\
\text { Bay }\end{array}$ & $\mathrm{H}_{2}$ & $\begin{array}{l}X \sim 0.13 \times \text { Beta }(0.57,0.53)+ \\
0.87 \times \text { Beta }(26.08,10.89)\end{array}$ & 0.885 & $>0.99$ \\
\hline b & $\begin{array}{l}\text { North } \\
\text { Bay }\end{array}$ & $\mathrm{H}_{2}$ & $\begin{array}{l}X \sim 0.07 \times \text { Beta }(3.48,66.21) \\
+0.93 \times \text { Beta }(10.99,2.89)\end{array}$ & 0.816 & $>0.99$ \\
\hline c & $\begin{array}{l}\text { North } \\
\text { Bay }\end{array}$ & $\mathrm{H}_{2}$ & $\begin{array}{l}X \sim 0.07 \times \operatorname{Beta}(13.97,10.32) \\
+0.93 \times \operatorname{Beta}(57.10,3.12)\end{array}$ & 0.007 & $>0.99$ \\
\hline d & $\begin{array}{l}\text { Ned's } \\
\text { Beach }\end{array}$ & $\mathrm{H}_{2}$ & $\begin{array}{l}X \sim 0.33 \times \text { Beta }(0.71,0.57)+ \\
0.67 \times \text { Beta }(108.55,68.91)\end{array}$ & 0.670 & $>0.99$ \\
\hline $\mathbf{e}$ & $\begin{array}{l}\text { Ned's } \\
\text { Beach }\end{array}$ & $\mathrm{H}_{2}$ & $\begin{array}{l}X \sim 0.51 \times \text { Beta }(16.58,4.28) \\
+0.49 \times \text { Beta }(114.8,2.32)\end{array}$ & 0.512 & 0.96 \\
\hline f & $\begin{array}{l}\text { Ned's } \\
\text { Beach }\end{array}$ & $\mathrm{H}_{2}$ & $\begin{array}{l}X \sim 0.55 \times \text { Beta }(0.90,0.34)+ \\
0.45 \times \text { Beta }(19.40,10.63)\end{array}$ & 0.444 & 0.93 \\
\hline
\end{tabular}

Model support is indicated by Akaike weights $\left(w_{i}\right)$, representing the conditional probability that a particular model provides the best fit of all candidate models (i.e. $\mathrm{H}_{0}, \mathrm{H}_{1}$ and $\mathrm{H}_{2}$ ). These give unambiguous support for a candidate if $>0.9$ (Johnson \& Omland 2004).

\subsection{Discussion}

The primary focus of this chapter was to establish a molecular and statistical framework by which to assess the incidence of hybridization between divergent Symbiodinium lineages. This is made difficult by their apparent haplontic life cycle, a lack of amenability to culture in many types (particularly in clade C Symbiodinium), and the paucity of well-characterized genetic markers. This study attempts to circumvent these obstacles by developing protocols to isolate and extract DNA from individual Symbiodinium cells, establish and quantify the dominant ribotype(s) within each genome, and test competing hypotheses explaining the observed pattern of intra-genomic variation. Using these techniques, a population of putative hybrids is identified inhabiting the reef building coral Pocillopora damicornis at the isolated, high-latitude reef of Lord Howe Island, Australia. 


\subsubsection{Method development}

The single-cell isolation and extraction method described here facilitated the rapid preparation of individual Symbiodinium cells prior to PCR (around 20 per hour), with the potential to be further improved with the application of flow-cytometry and fluorescence activated cell sorting (FACS). The protocol also showed good efficiency, with around $85 \%$ of isolated cells undergoing successful PCR amplification. The downstream application of DGGE and DNA sequencing successfully revealed the dominant ribotype(s) within individual cells, providing a reliable assessment of inter-genomic ITS2 diversity within the $P$. damicornis symbiont consortium. Used in conjunction with plasmid cloning, this method could be used to evaluate levels of intra-genomic variation in other genetic markers, providing an important assessment of their phylogenetic utility.

The qPCR assay developed in this study offers sufficient sensitivity to quantify ITS2 ratios at the subclade level. This represents a significant improvement in resolution from earlier cladelevel assays (Ulstrup \& van Oppen 2003; Loram et al. 2007a; Mieog et al. 2007; Correa et al. 2009; Yamashita et al. 2010; Cunning et al. 2013), since the subclade presents a more ecologically-relevant taxonomic unit (LaJeunesse 2005). This assay is also the first to quantify polymorphic rDNA sequences within individual Symbiodinium cells, and the second to do so in dinoflagellates (see also Brosnahan et al. 2010). This provides an important insight into the level of ITS2 variation within the Symbiodinium genome, underscoring concerns about its utility in establishing diversity estimates (Thornhill et al. 2007), and its suitability for quantifying the dynamics of mixed infections (Mieog et al. 2007). In particular, substantial differences in rDNA copy numbers observed between Symbiodinium types C100 and C109 highlight the pitfalls of using ITS2-qPCR to estimate abundance ratios of coexisting symbionts without single-cell validation.

Finally, the statistical methodology developed here identifies admixture in symbiont populations based on intra-genomic ITS2 ratios. Conflicting hypotheses of one, two and three coexisting populations were formulated, corresponding to the existence of a single symbiont clone harbouring a non-diagnostic polymorphism (NDP), the coexistence of two 'pure' (homogeneous) ribotypes, and mixed populations of genetically homogeneous and heterogeneous Symbiodinium cells, respectively. The model consistent with the latter hypotheses received unambiguous statistical support in all six $P$. damicornis colonies analysed. However, the model selection approach relies on forming a set of candidate models 
that are representative of the biological processes under investigation (Johnson \& Omland 2004). While the mixture model representing $\mathrm{H}_{2}$ is consistent with a population of hybrids coexisting with parental genotypes (progenitors), it cannot explicitly infer this scenario. This is because a similar pattern could arise from the incomplete concerted evolution of ancestral polymorphisms (ICEAP).

\subsubsection{Hybridization or incomplete concerted evolution of ancestral polymorphisms?}

The existence of both $\mathrm{C} 100$ and $\mathrm{C} 109$ ribotypes in the homogeneous condition affirms their status as diagnostic of separate Symbiodinium subclades (i.e. neither sequence represents a degenerating pseudo-gene). Furthermore, these two ribotypes differ at 5 variable nucleotide sites in the ITS2 region (2\% divergence), while NDPs typically feature a single nucleotide substitution or insertion/deletion (indel) that distinguish them from the dominant sequence variant (LaJeunesse 2005; Tonk et al. 2013). However, if both ribotypes were present within the genome of the most recent common ancestor of Symbiodinium C100 and C109, processes of concerted evolution may not have had sufficient time to homogenize the rDNA arrays of both taxa. Hence copies of the ribotype that is now diagnostic of the sister taxon may remain in the genome of one or both lineages. The Symbiodinium genome routinely hosts a diverse assemblage of ITS2 sequences (Thornhill et al. 2007), and several putative cases of ICEAP appear in the literature. For example, the ITS2 sequence diagnostic of Symbiodinium glynni (type D1) also occurs within the genome of S. trenchii (type D1a), with the incomplete displacement of a vestigial polymorphism invoked to explain their intra-genomic coexistence (Thornhill et al. 2007). However, several features of the data presented here suggest that an alternative explanation of hybridization should not be ruled out. First, the C100 and C109 sequences coalesce at the ancestral type $\mathrm{C} 3$, as opposed to either representing an intermediate evolutionary step toward the other (e.g. C103 and C118 in P. damicornis and C3hh and C3n in Seriatopora hystrix; see Figure 2.2b). If concerted evolution has not had sufficient time to homogenize all C109 rDNA repeats in the $\mathrm{C} 100$ genome, then vestigial copies of the intermediate $\mathrm{C} 3$ sequence would also likely persist as a non-dominant intra-genomic variant. Rather, the C3 sequence was not detected in any of the cells analysed, despite its characteristic DGGE band pattern (see Wicks et al. 2010, supplementary material). Second, concerted evolutionary processes rapidly homogenize intra-genomic co-dominance, either completely displacing a non-dominant polymorphism or leaving only background traces 
(Baldwin et al. 1995; Wendel et al. 1995; Ganley \& Scott 2002). This is inconsistent with the similar proportional abundance of ITS 2 polymorphisms within many of the genetically heterogeneous cells observed here, with more than a third of all symbionts featuring $\mathrm{C}_{\mathrm{C} 100}: \mathrm{C}_{\text {TOTAL }}$ ratios between 0.25 and 0.75 . Finally, frequency troughs along the $\mathrm{C}_{\mathrm{C} 100}: \mathrm{C}_{\text {TOTAL }}$ spectrum depict a degree of genetic isolation between genetically heterogeneous Symbiodinium cells and either of the 'pure' genotypes (i.e. homogeneous C100 and C109 cells), consistent with the substantial fitness loss often experienced by $\mathrm{F}_{2}$ and later-generation backcross genotypes (resulting from 'hybrid breakdown'; see Demuth \& Wade 2005).

While hybridization represents a plausible explanation for the intra-genomic co-dominance of the $\mathrm{C} 100$ and $\mathrm{C} 109$ ribotypes, the distinction between hybridization and ICEAP remains ambiguous. Addressing this question will likely require a significant investment of resources, including the use of WGA and/or the generation of isoclonal cultures (in order to facilitate multi-locus genotyping analysis on individual cells by targeting nuclear microsatellites and/or single nucleotide polymorphisms), and continued attempts to induce the sexual life cycle, both within and between cultured Symbiodinium lineages. Next generation sequencing (NGS) platforms also offer tremendous potential for identifying processes underlying intra-genomic variation in Symbiodinium, enabling the identification of rare sequence variants and the generation of high-volume sequence data from large numbers of individuals (Arif et al. 2014; Thomas et al. 2014; Green et al. 2014). Another area requiring investigation is the morphological, physiological and ecological characterization of putative Symbiodinium hybrids. Concerted evolution operates via a series of stochastic processes that occur independently of natural selection (Dover 1982). By contrast, hybridization is often accompanied by drastic changes in morphology, performance and fitness (Barton \& Bengtsson 1986; Barton 2001; Arnold 2007; Arnold \& Martin 2010), even involving diversification into new habitats (Rieseberg et al. 2003). Investigating the form, function, distribution and ecology of genetically heterogeneous Symbiodinium cells may therefore provide further insight into the incidence and potential evolutionary effects of hybridization in this genus. The remaining chapters of this thesis are concerned with addressing these objectives. 


\subsubsection{Background symbiont populations}

The results of this study indicate that at least three ITS2 genotypes can coexist within the symbiont consortium of $P$. damicornis (C100, C100/C109 and C109). While homogeneous Symbiodinium C109 cells were only ever detected at background levels (constituting less than $7 \%$ of the symbiont population, and possibly suggesting a non-homologous relationship with this host species), the biological relevance of this population may extend well beyond providing a presumably minor contribution to the overall productivity of the symbiosis. Genetically heterogeneous Symbiodinium cells outnumbered 'pure' genotypes in more than half of the colonies sampled, suggesting that rare sexual reproduction events between $\mathrm{C} 100$ and C109 may facilitate asexual hybrid proliferation, with potentially important functional implications for the coral colony. The evolutionary contribution of Symbiodinium C109 may be more important still, if hybrids create a 'bridge' for the migration of genetic material to the dominant C100 lineage (i.e. introgression; see Figure 2.1b). A small number of genetically heterogeneous symbionts featured $\mathrm{C}_{\mathrm{C} 100}: \mathrm{C}_{\mathrm{TOTAL}}$ ratios near 0.75 , and thus potentially represent $\mathrm{F}_{1} \times \mathrm{C} 100$ backcross genotypes. However, this pattern could equally have arisen from ICEAP, differential rDNA inheritance in $\mathrm{F}_{1}$ hybrids (arising from dissimilar copynumbers between parent taxa; e.g. Brosnahan et al. 2010), or even concerted evolution acting to homogenize rDNA variability in the hybrid genome (e.g. Wendel et al. 1995). Establishing the incidence of introgression would initially require the identification of individual hybridand backcross classes (i.e. $\mathrm{F}_{1}$ hybrid, $\mathrm{F}_{2}$ backcross, etc.). This in turn requires the genotyping of a large number of individuals, and the analysis of at least 13-50 ancestry-informative loci per individual (Epifanio \& Phillipp 1997; Fitzpatrick 2012). This project is not sufficiently resourced to carry out such a comprehensive task; however it does serve to highlight the perils of dismissing symbionts that persist in low abundance as biologically-irrelevant or simply representing surface contamination.

\subsubsection{Conclusion}

While the results presented in this chapter do not provide unequivocal evidence of hybridization between divergent Symbiodinium lineages, they provide an initial 'proof of principle' for its occurrence. In doing so, this study draws attention to the important evolutionary implications that may accompany the generation of new genetic diversity in Symbiodinium, including the potential for rapid symbiont adaptation through introgression. 
Progress in this area has been hindered by a lack of available methodology, an obstacle that is addressed here through the development of new molecular and statistical methods focussed on the individual Symbiodinium cell. Through the application of these techniques, colonies of P. damicornis inhabiting the reef of Lord Howe Island are identified as representing a putative Symbiodinium 'hybrid zone', requiring further molecular, morphological, physiological and ecological assessment. Additional development of this research may help to characterize and predict the evolutionary response of the coral-algal symbiosis to the many anthropogenic impacts currently threatening the world's coral reefs. 


\section{Chapter 3: The spatiotemporal distribution of putative Symbiodinium hybrids}

\subsection{Introduction}

Providing unequivocal evidence of hybridization has proven difficult in many species. In particular, the persistence of ancestral polymorphisms can leave a similar genomic signature to that of hybridization, and hence distinguishing between these fundamentally different evolutionary processes requires corroborative population-genetic, morphological, physiological and ecological evidence (Vriesendorp \& Bakker 2005; Willis et al. 2006). Ecological data can be particularly useful for assessing the candidacy of genetically additive individuals as putative hybrids. While concerted evolutionary processes act independently of natural selection (Dover 1982), the ability of hybrids to survive and reproduce is strongly influenced by their biotic and abiotic environment (Lewontin \& Birch 1966). As such, hybrids are generally more common at the ecological and/or geographical margin of the parental species range, where selection arising from competition with parents is weak (Arnold 1997; Rieseberg 1997), and opportunities exist for 'transgressive' hybrids to diversify into extreme habitats (see below).

The lack of competition and extreme abiotic conditions found in marginal habitats can create opportunities for hybrids to diversify into vacant niches, through a process called 'transgressive segregation'. When individuals from two divergent lineages cross, the majority of the many possible hybrid recombinants are generally inviable or unfit, since 'hybrid breakdown' arises from the segregation of co-adapted gene complexes or the creation of maladapted gene combinations (Barton 2001; Demuth \& Wade 2005). However, the genetic variation created by hybridization can occasionally produce a fit genotype, that may escape this 'rat race' of unfit congeners (Seehausen 2004; Arnold \& Martin 2010). Transgressive segregation, the emergence of 'extreme' hybrid phenotypes via recombination (Rieseberg et al. 1999, 2000; Seehausen 2004; Bell \& Travis 2005), can enable hybrids to exploit new environments and even develop into stable, reproductively-isolated lineages (Rieseberg et al. 2003; Gompert et al. 2006). The ability of a fit hybrid recombinant to reproduce asexually can further increase its chances of establishing a foot-hold in a vacant niche, from where it may rapidly proliferate, stabilize, and adapt to the new conditions (Rieseberg et al. 2003). 
The best-studied example of hybrid diversification via transgressive segregation occurs in the wild sunflower genus Helianthus. The hybrid species $H$. paradoxus acquired a particular combination of alleles from $H$. annuus and $H$. petiolaris that conferred additive phenotypic effects, resulting in substantial increases in succulence, calcium uptake, and sodium exclusion relative to both progenitor species (Lexer et al. 2003). Positive directional selection acting on these adaptations enabled the hybrid lineage to diversify into extremely saline marshes (Lexer et al. 2003; Rieseberg et al. 2003), where neither parental species can persist (Welch \& Rieseberg 2002). Another hybrid species, Helianthus deserticola, diversified into arid areas by acquiring several transgressive 'desert adaptations', including reduced boron uptake, reduced leaf size, and rapid flowering (Rieseberg et al. 2003). Hybridization has thus been implicated in major ecological transitions, with hybrids commonly found beyond the range margins of parental taxa (Wang et al. 1990; Rieseberg 1991; Morrell \& Rieseberg 1998; Rieseberg et al. 2003; Barrett et al. 2007; Hamilton et al. 2009).

Marginal coral reefs may provide a suitable environment in which hybrid diversification could occur. These habitats are characterized by extreme conditions such as atypically high or low temperatures, irradiance levels outside those required for optimum photosynthesis, and/or a reduction in quality of other parameters such as salinity, nutrient loads or $\mathrm{pH}$ (Kleypas et al. 1999). The world's southernmost coral reef at Lord Howe Island (Australia; $31^{\circ} 33^{\prime} \mathrm{S}$ ) is situated at the transition zone between coral- and macroalgal-dominated benthic assemblages (Allen \& Paxton 1974), and is regularly exposed to wide fluctuations in temperature, light and nutrient levels (Harriott \& Banks 2002). The extreme abiotic conditions at LHI reef may therefore provide vacant adaptive peaks that select for extreme hybrid phenotypes. Indeed, the lack of evidence for hybridization among other Symbiodinium taxa despite several multilocus genotyping studies (e.g. Santos et al. 2003b; Pettay et al. 2011; Thornhill et al. 2013a; LaJeunesse et al. 2014), suggests that if hybridization does occur in symbiotic dinoflagellates, it may well be confined to isolated, marginal habitats such as LHI.

This chapter aimed to assess the candidacy of genetically heterogeneous Symbiodinium cells as putative hybrids by examining their ecological characteristics and distribution patterns on the LHI reef. A bulk-cell qPCR method was developed to quantify the number of genetically heterogeneous Symbiodinium cells (putative hybrids) as a proportion of the total number of symbionts in the P. damicornis consortium (Symbiodinium HETEROGENEOUs: Symbiodinium тотAL, hereafter abbreviated to $S_{\mathrm{H}}: S_{\mathrm{T}}$ ). Surveys of $S_{\mathrm{H}}: S_{\mathrm{T}}$ were carried out over three nested spatial scales and two summer-winter cycles. Three separate hypotheses were tested to 
explain the spatial and temporal distribution of putative Symbiodinium hybrids. First, $S_{\mathrm{H}}: S_{\mathrm{T}}$ varies within and between $P$. damicornis colonies, and is additionally variable between geographically isolated reef sites. Second, $S_{\mathrm{H}}: S_{\mathrm{T}}$ varies within colonies through time, in response to cyclical changes in the abiotic environment. Third, site-averaged $S_{\mathrm{H}}: S_{\mathrm{T}}$ values correlate with one or more of a suite of environmental variables obtained from long-term environmental monitoring at the study sites.

\subsection{Methods}

\subsubsection{Study location and species}

This study was carried out at the high-latitude, marginal coral reef at Lord Howe Island, Australia $(-31.5,159.1)$. The host species investigated was Pocillopora damicornis, a widely distributed reef-building coral that forms a dominant component of the LHI reef (Veron 2000; Miller \& Ayre 2004). P. damicornis was previously shown to host Symbiodinium C100, C103 or C118, with the majority of colonies hosting C100 (Wicks et al. 2010). However, single-cell analysis revealed that these colonies also host a cryptic population of Symbiodinium C109 (Genbank accession number KJ530690; see chapter 2), and a third population of genetically heterogeneous symbionts, featuring both $\mathrm{C} 100$ and $\mathrm{C} 109$ ribotypes (see chapter 2). These symbionts may represent C100 × C109 hybrids, or alternatively, Symbiodinium cells that host a persistent ancestral polymorphism.

\subsubsection{Coral sampling and preservation}

To analyse the spatial distribution of genetically heterogeneous Symbiodinium cells, and determine whether associations exist between local temperature conditions and their proportional abundance within the symbiont consortium $\left(S_{\mathrm{H}}: S_{\mathrm{T}}\right)$, five $P$. damicornis colonies were sampled from each of eight LHI reef sites (Figure 3.1). Three terminal fragments were removed (using diagonal pliers) from each colony, preserved in DMSO preservation buffer (20\% DMSO, 250 mM EDTA, NaCl saturated, pH 8.0; Seutin et al. 1991) and stored at -20 ${ }^{\circ} \mathrm{C}$ prior to DNA extraction. Colonies were sampled as near as possible to the in situ temperature data loggers operated by the Australian Institute of Marine Science (AIMS); however, a spacing of at least $2 \mathrm{~m}$ was maintained between colonies to reduce the risk of 
sampling ramets. To explore the relationship between $S_{\mathrm{H}}: S_{\mathrm{T}}$ and depth (a proxy for irradiance), a more exhaustive sampling effort was carried out at a single reef margin site featuring a steep sloping profile (The Arch, where P. damicornis colonies occur from approximately 4-12 m). Twenty one additional colonies were sampled as above at roughly even intervals along the depth gradient, with their apical depth recorded using a dive computer (Suunto Gekko). Samples were collected within the space of $\sim 10 \mathrm{~min}$; therefore negligible differences in tidal height were assumed.

For temporal analysis of $S_{\mathrm{H}}: S_{\mathrm{T}}$, numbered cattle ear-tags were attached to five colonies from each of the three inner-lagoon sites (Sylph's Hole, North Bay and Comet's Hole) during March 2011. A single branch tip was sampled from each tagged colony during March 2011, September 2011, March 2012 and September 2012 (six-monthly sampling over 1.5 years). These sampling times were chosen to closely follow the thermal maxima and minima over two seasonal cycles, and hence establish the relationship between the relative abundance of putative hybrids and natural cyclical changes in seawater temperature. 


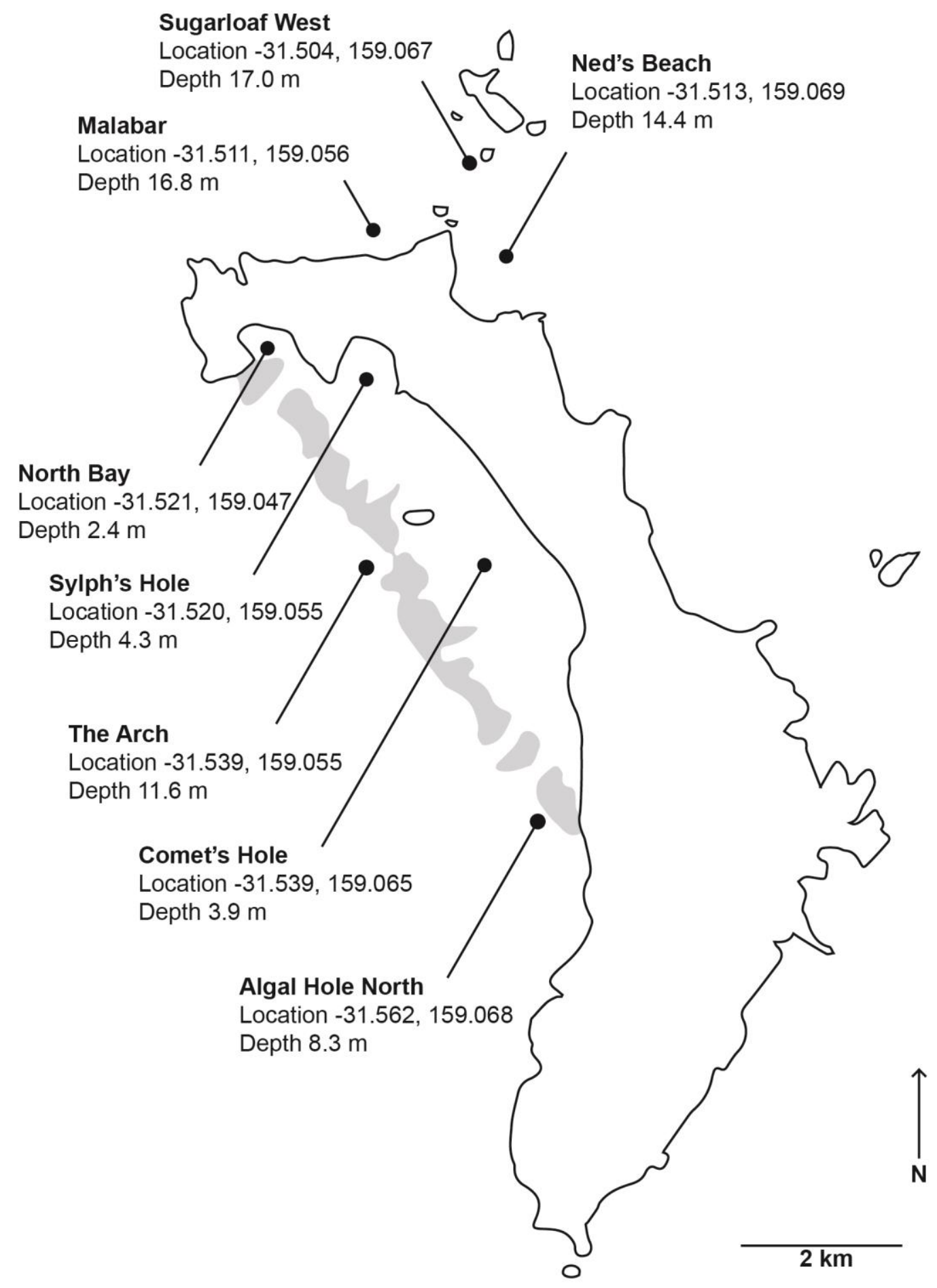

Figure 3.1 Locations of study sites at Lord Howe Island

Spatial surveys were carried out at the eight sites indicated. Temporal surveying was carried out at Comet's Hole, North Bay and Sylph's Hole, at six-monthly intervals from March 2011 to September 2012. Australian Institute of Marine Science (AIMS) data loggers are permanently deployed at all sites except The Arch, where a HOBO pendant logger was deployed from September 2011 to September 2012. Logger depths and coordinates are shown for each site. 


\subsubsection{Extraction of bulk-cell Symbiodinium DNA}

Preserved coral tissue was separated from the underlying skeleton by centrifugation through a fine plastic-coated wire mesh (grid size $1 \mathrm{~mm} ; 16,100 \mathrm{x}$ g for $10 \mathrm{~s}$ ). The skeleton was discarded and $200 \mu 1$ of DMSO buffer were added to the tissue homogenate. The sample was then vortexed and centrifuged for $10 \mathrm{~min}$ at $16,100 \mathrm{x}$ g to separate the host and symbiont fractions. The supernatant (host fraction) was discarded and a further $200 \mu 1$ DMSO buffer were added to the Symbiodinium pellet, which was re-suspended by milling without beads for $3 \mathrm{~min}$ at $50 \mathrm{~Hz}$ (Qiagen TissueLyser LT; Qiagen). A $10 \mu \mathrm{l}$ sub-sample was added to a $1.7 \mathrm{ml}$ micro-centrifuge tube containing $50 \mathrm{mg}$ acid-washed glass beads (710-1180 $\mu \mathrm{m}$; SigmaAldrich), and milled for $3 \mathrm{~min}$ at $50 \mathrm{~Hz}$ to disrupt the Symbiodinium cells and release nucleic acids. Following a $10 \mathrm{~min}$ incubation period at $4{ }^{\circ} \mathrm{C}, 90 \mu \mathrm{l}$ of de-ionised water were added. The sample was then vortexed and centrifuged for $10 \mathrm{~min}$ at $16,100 \mathrm{x} \mathrm{g}$ to pellet cellular debris. The DNA-enriched supernatant $(50 \mu \mathrm{l})$ was transferred to a new micro-centrifuge tube with an equal volume of 2-propanol for precipitation of nucleic acids, and centrifuged for 10 min at $16,100 \mathrm{x} g$ to collect the DNA pellet. The supernatant was discarded and $200 \mu 1$ of wash buffer $(70 \% \mathrm{v} / \mathrm{v}$ ethanol) were added. The sample was vortexed and returned to the centrifuge for a further $10 \mathrm{~min}$ at $16,100 \mathrm{x}$. The supernatant was carefully removed with a pipette and the DNA pellet dried under a laminar flow hood for $30 \mathrm{~min}$. Elution buffer (10 $\mathrm{mM}$ Tris- $\mathrm{HCl} ; 0.1 \mathrm{mM}$ EDTA; $\mathrm{pH}=8.0$ ) was then added to a final volume of $50 \mu 1$. Following a 10 min re-hydration period, the DNA was eluted by milling without beads for 1 $\min$ at $30 \mathrm{~Hz}$.

\subsubsection{PCR and electrophoresis}

Denaturing gradient gel electrophoresis (DGGE) was carried out on all samples to establish the presence or absence of Symbiodinium types other than C100 and C109. PCR amplification for denaturing gradient gel electrophoresis (DGGE) was carried out with the primers ITSintfor 2 and ITS2CLAMP (LaJeunesse 2002). Thermal cycling involved an initial denaturation step of $3 \mathrm{~min}$ at $95{ }^{\circ} \mathrm{C}$ followed by 40 cycles of $15 \mathrm{~s}$ at $95{ }^{\circ} \mathrm{C}, 15 \mathrm{~s}$ at $56{ }^{\circ} \mathrm{C}$ and $10 \mathrm{~s}$ at $72{ }^{\circ} \mathrm{C}$ (Applied Biosystems Veriti thermo-cycler; Life Technologies). Each PCR reaction contained $4 \mu \mathrm{l}$ of DNA template solution, 1x MyTaq PCR reaction mix (Bioline), 10 pmol each primer, $10 \mu \mathrm{g}$ bovine serum albumin (BSA; Sigma) and deionised sterile water to a total volume of $20 \mu \mathrm{l}$. A template-free control was included with each run. To ensure the 
final product was within the intended size range (300-350 bp) and that amplification did not occur in the template-free control reaction, $5 \mu$ of the final PCR product were electrophoresed on a $1.5 \%$ agarose gel containing 1x SYBR safe nucleic acid stain (Life Technologies) alongside a DNA standard (Hyperladder ${ }^{\mathrm{TM}}$ II; Bioline). Agarose gels were viewed and imaged on a blue light trans-illuminator (Safe Imager; Invitrogen). The remaining PCR product was loaded on a $200 \times 200 \times 1 \mathrm{~mm}, 8 \%$ denaturing polyacrylamide gel (25-50\% denaturant gradient), and run in $1 \times$ TAE at $150 \mathrm{~V}$ for $7 \mathrm{~h}$ at $60{ }^{\circ} \mathrm{C}$ (DCode system; BioRad) alongside known ITS2 sequences of Symbiodinium C100 and C109 (extracted from plasmid DNA; see chapter 2 for cloning methods). Following electrophoresis, denaturing gels were stained with ethidium bromide and viewed on a UV trans-illuminator (FirstLight; UVP) and the presence or absence of each ITS2 sequence type was recorded.

\subsubsection{Quantification of genetically heterogeneous Symbiodinium cells}

To determine the proportional abundance of ITS2 type $\mathrm{C} 100\left(\mathrm{C}_{\mathrm{C} 100}: \mathrm{C}_{\mathrm{TOTAL}}\right)$ in bulk-cell Symbiodinium samples, quantitative PCR (qPCR) was carried out with the universal primers CInnerFor and CInnerRev, and the TaqMan fluorogenic probes $\mathrm{C}_{100}{ }^{+}$and $\mathrm{C} 100^{-}$(see chapter 2 for nucleotide sequences). Each qPCR reaction contained $4 \mu 1$ DNA template, 1x TaqMan Universal Mastermix II (Life Technologies), 18 pmol each primer, 1x TaqMan fluorogenic probe (Life Technologies), $10 \mu \mathrm{g}$ BSA (Sigma-Aldrich) and deionised sterile water to a total volume of $20 \mu \mathrm{l}$. Thermal cycling involved an initial $10 \mathrm{~min}, 95^{\circ} \mathrm{C}$ denaturation step followed by 40 cycles of $15 \mathrm{~s}$ at $95{ }^{\circ} \mathrm{C}$ and $1 \mathrm{~min}$ at $60{ }^{\circ} \mathrm{C}$ (Applied Biosystems StepOne quantitative PCR instrument; Life Technologies). $C_{t}$ values were determined as the cycle at which the change in fluorescence was significantly different to the background level $\left(\Delta R_{n}=\right.$ 0.05; obtained using the instrument's built-in algorithm). Reactions featuring $\mathrm{C}_{\mathrm{t}}$ values below the standard curve intercept (36.873 and 37.119 for $\mathrm{C}_{100^{+}}$and $\mathrm{C} 100^{-}$assays, respectively; see Appendix $B$ ) and sufficiently low standard deviations $(<0.5)$ were included in the analysis. Plasmid DNA template solutions containing pure Symbiodinium C100 and C109 sequences were used to construct standard curves (see chapter 2 for cloning methods). To establish standard curve parameter estimates, 6 logarithm serial dilutions of C100 and C109 plasmid DNA were run in duplicate $\left(10 \mathrm{pg} \mathrm{l}^{-1}\right.$ to $0.1 \mathrm{fg} \mathrm{\mu l}^{-1}$; corresponding to approximately 2,120,000 and 21 ITS2 copies $\mu 1^{-1}$, respectively). DNA template solutions generated from bulk-cell Symbiodinium samples were run in duplicate with a template-free control included 
for each probe type. The total number of ITS 2 copies $\left(\mathrm{C}_{\text {TOTAL }}\right)$ within each sample was estimated as the sum of the $\mathrm{C} 100$ copy number $\left(\mathrm{C}_{\mathrm{C} 100}\right)$ and the number of Symbiodinium clade C ITS2 sequences other than type C100 (as determined by mean $\mathrm{C}_{\mathrm{t}}$ values derived from $\mathrm{C} 100^{+}$and $\mathrm{C} 100^{-}$assays, respectively). The proportion of total Symbiodinium clade C sequences that were of type $\mathrm{C} 100$ was expressed as the bulk-cell $\mathrm{C}_{\mathrm{C} 100}: \mathrm{C}_{\mathrm{TOTAL}}$ ratio.

The proportional abundance of genetically heterogeneous Symbiodinium cells $\left(S_{\mathrm{H}}: S_{\mathrm{T}}\right)$ was derived from the bulk-cell $\mathrm{C}_{\mathrm{C} 100}: \mathrm{C}_{\mathrm{TOTAL}}$ ratio using parametric information generated from single-cell qPCR analysis carried out in chapter 2 . For each of the six colonies sampled in the chapter 2 analysis, the proportion of individuals assigned to the heterogeneous mixture-model component (see Table 2.2) was plotted against the bulk-cell $\mathrm{C}_{\mathrm{C} 100}: \mathrm{C}_{\mathrm{TOTAL}}$ ratio. A downward parabola was fitted to the bivariate data (form $\mathrm{y}=a \mathrm{x}^{2}+b \mathrm{x} ; a<0$; where $\mathrm{y}=S_{\mathrm{H}}: S_{\mathrm{T}} ; \mathrm{x}=$ $\mathrm{C}_{\mathrm{C} 100}: \mathrm{C}_{\mathrm{TOTAL}}$ ) using the curve fitter function in Sigmaplot v11.0 (Systat). The bulk-cell $\mathrm{C}_{\mathrm{C} 100}: \mathrm{C}_{\mathrm{TOTAL}}$ ratio approximated $S_{\mathrm{H}}: S_{\mathrm{T}}$ via the second-order polynomial relationship y = $3.4484 x^{2}+3.3604 x$ (Figure 3.2). The parameters generated in this analysis were then used to predict $S_{\mathrm{H}}: S_{\mathrm{T}}$ from bulk-cell ITS2 ratios obtained from each coral sample in the spatiotemporal distribution analysis.

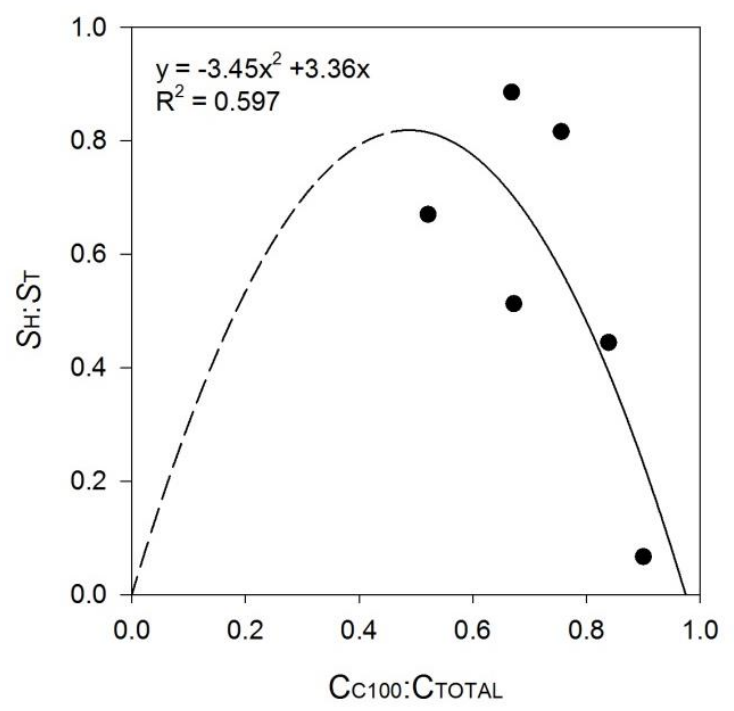

Figure 3.2 Estimating the proportional abundance of putative Symbiodinium hybrids The application of bulk-cell qPCR enabled the rapid approximation of $S_{\mathrm{H}}: S_{\mathrm{T}}$ without the need for single-cell analysis. The qPCR-generated $\mathrm{C}_{\mathrm{C} 100}: \mathrm{C}_{\text {TOTAL }}$ ratio (bulk-cell) predicted the $S_{\mathrm{H}}: S_{\mathrm{T}}$ values generated in chapter 2 via the polynomial relationship $\mathrm{y}=-3.45 \mathrm{x}^{2}+3.36 \mathrm{x}$. The curve section from $\mathrm{x}=0$ to $\mathrm{x}=0.5$ is shown by a dashed line, since none of the six colonies analysed in chapter 2 or the $61 P$. damicornis colonies analysed in the present chapter produced $\mathrm{C}_{\mathrm{C} 100}$ : $\mathrm{C}_{\text {TOTAL }}$ ratios less than 0.5 . 


\subsection{6. $\quad$ Water temperature monitoring}

To determine whether the distribution of putative Symbiodinium hybrids is associated with the local temperature regime, environmental data were obtained from the in situ data loggers operated by the Australian Institute of Marine Science (AIMS). Monthly average temperatures from March 2009 to August 2012 were downloaded from the AIMS public website (accessed from http://www.data.aims.gov.au/ on 20/03/2014). These loggers continuously record seawater temperatures at Algal Hole North, Comet's Hole, Malabar, Ned's Beach, North Bay, Sugarloaf West and Sylph's Hole (Figure 3.1). An eighth logger also permanently monitors water temperatures at Wheatsheaf Islet (adjacent to Ball's Pyramid, $20 \mathrm{~km}$ to the southeast of Lord Howe Island); however P. damicornis colonies were not found at this site despite a thorough sampling effort. AIMS loggers are occasionally retrieved for battery replacement or repairs (I. Kerr, LHIMPA, pers. comm.). To correct for bias associated with these logging gaps, sites featuring missing data were paired with their most proximal site for which logging was continuous from March 2009 to August 2012. A correlation analysis was carried out using the available simultaneous measurements, and missing data were estimated using the slope and intercept of the fitted line (see Appendix B). In lieu of an AIMS logger at The Arch, a HOBO pendant logger (Onset Computer Corporation) was deployed at a depth of $11.6 \mathrm{~m}$ from 18/09/2011 to 31/08/2012 (measuring and logging temperature at 15 min intervals). Data obtained from this instrument were visually checked for spurious values, month-averaged and temporally aligned with temperature values measured by the AIMS logger deployed at Algal Holes (for which continuous month-averaged temperatures were available from March 2009 to August 2012, inclusive). Month-averaged temperatures at The Arch prior to September 2011 were estimated using correlation analysis as described above, with Algal Hole North used as the predictor site (see Appendix $B$ ). For each of the study sites, a four-parameter sine function of the form

$$
y=a \sin \left(\frac{2 \pi x}{b}-c\right)+d
$$

Equation 3.1

was fitted to monthly mean temperature data using the curve fitter function in Sigmaplot v11.0 (Systat). The fitted temperature mean $(d)$, maximum $(d+a)$, minimum $(d-a)$, and range $(2 a)$ were extracted as candidate predictor variables for regression against site-averaged $S_{\mathrm{H}}: S_{\mathrm{T}}$ ratios. 


\subsubsection{Statistical analysis}

Spatiotemporal variation in $S_{\mathrm{H}}: S_{\mathrm{T}}$ was assessed using a combination of repeated measures analysis of variance (rmANOVA), nested univariate ANOVA and linear regression. Spatial variability in $S_{\mathrm{H}}: S_{\mathrm{T}}$ within and between $P$. damicornis colonies was modelled using twofactor nested ANOVA, with Site (eight levels, random) and Colony(Site) (colony nested within site; five levels, random) as hierarchical factors and Branch as the residual. Temporal variation in $S_{\mathrm{H}}: S_{\mathrm{T}}$ within and between colonies was assessed using rmANCOVA with Time as a within-subject factor (four levels, fixed), Site as a between-subject factor (3 levels, random) and Colony as the residual. Relative variance components for random effects were estimated using the MINQUE procedure (Rasch \& Mašata 2006). In order to assess the effect of depth (a proxy for irradiance) on $S_{\mathrm{H}}: S_{\mathrm{T}}$ within colonies, a linear regression analysis was carried out with colony- $S_{\mathrm{H}}: S_{\mathrm{T}}$ as the dependent variable and depth (in metres) as the explanatory variable. To establish the best predictor(s) for site-averaged $S_{\mathrm{H}}: S_{\mathrm{T}}$, a multiple linear regression analysis was carried out using stepwise addition of candidate explanatory temperature variables (mean, minimum, maximum and range). For each linear model analysis, assumptions of variance homoscedasticity and residual normality were validated using Levene's and Shapiro-Wilk tests, respectively. Statistical analyses were carried out using R v2.13.2 (R Development Core Team 2011) and SPSS Statistics v20 (IBM).

\subsection{Results}

\subsubsection{Spatiotemporal distribution patterns}

Investigating the spatiotemporal distribution of putative hybrid symbionts involved the analysis of 186 individual branches taken from a total of $61 P$. damicornis colonies, sampled from eight reef sites around Lord Howe Island. Symbiodinium C100 represented the dominant ITS2 type in all colonies and branches analysed. Approximately two thirds of the colonies also produced a non-dominant DGGE band representative of Symbiodinium C109 (41 colonies; see Appendix B). No other Symbiodinium sequences were detected in this study, including the rare types $\mathrm{C} 103$ and $\mathrm{C} 118$ previously identified from $P$. damicornis at LHI (Wicks et al. 2010). This may arise from differences in host-identification specificity between studies. For example, two ambiguous Sylph's Hole colonies omitted from the present study 
(that appeared to be the $P$. damicornis $\times$ Stylophora pistillata hybrids described by Miller $\&$ Ayre 2004) were later found to exclusively host Symbiodinium C118 (unpublished data).

The proportional abundance of genetically heterogeneous Symbiodinium cells within colonies $\left(S_{\mathrm{H}}: S_{\mathrm{T}}\right.$ ) ranged from 0.10 to 0.82 (corresponding to bulk-cell $\mathrm{C}_{\mathrm{C} 100}: \mathrm{C}_{\mathrm{TOTAL}}$ ratios of 0.9438 and 0.516, respectively; see Appendix $B$ ). The $\mathrm{C}_{\mathrm{C} 100}: \mathrm{C}_{\text {TOTAL }}$ ratio was generally reflected in the relative intensity of C100- and C109-diagnostic bands observable on the DGGE gel. Both bands were universally visible in samples where genetically heterogeneous cells constituted more than $60 \%$ of the symbiont consortium $\left(S_{\mathrm{H}}: S_{\mathrm{T}}>0.6 ; \mathrm{C}_{\mathrm{C} 100}: \mathrm{C}_{\mathrm{TOTAL}}<0.74\right)$, while the C109-diagnostic band was either not visible or difficult to detect in samples where less than half of the resident symbionts were classified as heterogeneous $\left(S_{\mathrm{H}}: S_{\mathrm{T}}<0.5 ; \mathrm{C}_{\mathrm{C} 100}: \mathrm{C}_{\mathrm{TOTAL}}>\right.$ 0.78; see Appendix B). Samples featuring an intermediate proportional abundance of genetically heterogeneous cells $\left(0.6<S_{\mathrm{H}}: S_{\mathrm{T}}<0.5\right)$ either produced a DGGE profile diagnostic of $\mathrm{C} 100$ in isolation or that of both $\mathrm{C} 100$ and $\mathrm{C} 109$, underscoring the limitations of applying this method for DNA quantitation.

The distribution of putative Symbiodinium hybrids was variable between colonies over spatial scales of metres to tens of metres, and even more so between sites separated by hundreds to thousands of metres. The majority of spatial variation in $S_{\mathrm{H}}: S_{\mathrm{T}}$ partitioned at the level of Colony (Site) (colony nested within site; $\mathrm{F}_{32,80}=58.76 ; \mathrm{p}<0.001 ; 72 \%$ of total variance explained; Table 3.1), with colony-averaged $S_{\mathrm{H}}: S_{\mathrm{T}}$ values ranging from 0.142 to 0.813 . A significant proportion of spatial variation also partitioned at the Site level (24\% of total variance explained; $\mathrm{F}_{7,32}=2.72 ; \mathrm{p}=0.025 ;$ Table 3.1), with site-averaged $S_{\mathrm{H}}: S_{\mathrm{T}}$ ratios ranging from 0.323 (Sylph's Hole) to 0.613 (Ned's Beach). However, the proportional abundance of genetically heterogeneous Symbiodinium cells was highly conserved between branches within colonies (4\% residual variance component; Table 3.1). Furthermore, $S_{\mathrm{H}}: S_{\mathrm{T}}$ did not vary within colonies through time. A longitudinal survey of 15 tagged P. damicornis colonies at North Bay, Sylph's Hole and Comet's Hole over two summer winter cycles showed a distinct lack of temporal variation in the proportional abundance of genetically heterogeneous Symbiodinium cells (Pillai's Trace $\mathrm{p}=0.509$ and 0.217 for Time $\mathrm{x}$ Site interaction and Time main effect, respectively; Table 3.2). When reduced to a two-factor nested ANOVA model $\left(S_{\mathrm{H}}: S_{\mathrm{T}} \sim\right.$ Site + Colony $($ Site $) ;$ Branch as the residual with temporal replication), the error variance accounted for a similar proportion of the total variance in $S_{\mathrm{H}}: S_{\mathrm{T}}$ as was explained in the original spatially-nested analysis (3.8\%; Figure 3.4a; Table 3.3). 
Table 3.1 Nested ANOVA for spatial distribution of putative Symbiodinium hybrids

\begin{tabular}{llllllll}
\hline Source of variation & SS & $\begin{array}{l}\text { Hypothesis } \\
\text { df }\end{array}$ & $\begin{array}{l}\text { Error } \\
\text { df }\end{array}$ & $\boldsymbol{F}$ & $\boldsymbol{P}$ & $\begin{array}{l}\text { Variance } \\
\text { component }\end{array}$ & $\begin{array}{l}\text { Variance } \\
\text { explained }\end{array}$ \\
\hline Between sites & 1.048 & 7 & 32 & 2.718 & $<0.001$ & 0.006 & $24 \%$ \\
Between colonies within sites & 1.763 & 32 & 80 & 58.761 & 0.025 & 0.018 & $72 \%$ \\
Between branches within colonies (residual) & 0.075 & 80 & & & & 0.001 & $4 \%$ \\
\hline
\end{tabular}

Table 3.2 Repeated measures ANOVA for temporal changes in putative hybrid abundance

\begin{tabular}{lllllll}
\hline Source of variation & & SS & Hypothesis df & Error df & $\boldsymbol{F}$ & $\boldsymbol{P}$ \\
\hline Within colonies & Time & 0.002 & 3 & 36 & 1.112 & 0.357 \\
& Time $\times$ Site & 0.005 & 6 & 36 & 1.180 & 0.339 \\
Between colonies & Site & 0.029 & 2 & 12 & 0.576 & 0.577 \\
\hline
\end{tabular}

Table 3.3 Reduced nested ANOVA for temporal changes in putative hybrid abundance

\begin{tabular}{llllllll}
\hline Source of variation & SS & $\begin{array}{l}\text { Hypothesis } \\
\text { df }\end{array}$ & Error df & $\boldsymbol{F}$ & $\boldsymbol{P}$ & $\begin{array}{l}\text { Variance } \\
\text { component }\end{array}$ & $\begin{array}{l}\text { Variance } \\
\text { explained }\end{array}$ \\
\hline Between inner-lagoon sites & 0.114 & 2 & 12 & 0.576 & 0.577 & $0^{*}$ & $0 \%$ \\
Between colonies within inner-lagoon sites & 1.191 & 12 & 45 & 150.242 & $<0.001$ & 0.025 & $96.2 \%$ \\
$\begin{array}{l}\text { Between time-points within colonies } \\
\text { (residual) }\end{array}$ & 0.030 & & & & & 0.001 & $3.8 \%$ \\
\hline *Rounded to zero, since estimated variance component was negative & & & & & & \\
\hline
\end{tabular}

*Rounded to zero, since estimated variance component was negative 


\subsubsection{Environmental niche partitioning}

Thermal characteristics were variable within and between sites, with the inner-lagoon Sylph's Hole reef experiencing the highest level of seasonal temperature variability (Figure 3.3). This poorly-flushed site experienced the warmest summer maximum and coolest winter minimum during the logging period (March 2009 to August 2012; fitted values $=17.56{ }^{\circ} \mathrm{C}$ and 25.18 ${ }^{\circ} \mathrm{C}$, respectively). Monthly averages and raw data values were occasionally more extreme than the fitted maxima and minima of the climate model, particularly during the anomalously warm summer of 2009/2010 (Figure 3.3). Monthly temperature averages at Sylph's Hole ranged from $17.06{ }^{\circ} \mathrm{C}$ in August 2012 to $26.25^{\circ} \mathrm{C}$ in January 2010, while actual 10-min logging values at this site ranged from $14.91{ }^{\circ} \mathrm{C}$ during July 2011 to $28.05{ }^{\circ} \mathrm{C}$ during January 2010. The absolute maximum temperature of $28.16^{\circ} \mathrm{C}$ was recorded at Comet's Hole during January 2010. Of the four candidate predictor variables assessed in the stepwise multiple regression analysis (model-fitted temperature mean, minimum, maximum, and range), the fitted thermal maximum emerged as the sole significant predictor of $S_{\mathrm{H}}: S_{\mathrm{T}}\left(\mathrm{F}_{1,6}=23.137 ; \mathrm{p}=\right.$ 0.003; Figure 3.4b-e). The linear relationship between maximum temperature and $S_{\mathrm{H}}: S_{\mathrm{T}}$ featured a strong negative correlation $\left(\mathrm{R}^{2}=0.794\right)$, with a $0.5{ }^{\circ} \mathrm{C}$ increase in the thermal maximum (from $24.7^{\circ} \mathrm{C}$ to $25.2^{\circ} \mathrm{C}$ ) corresponding to a $35 \%$ reduction in $S_{\mathrm{H}}: S_{\mathrm{T}}$. Finally, no clear correlation between $S_{\mathrm{H}}: S_{\mathrm{T}}$ and depth was evident among 26 P. damicornis colonies sampled along their natural bathymetric distribution at a sloping reef-margin site (The Arch; linear regression, $F_{1,24}=2.222 ; p=0.149 ; R^{2}=0.085$; Figure $3.4 b$ ). 


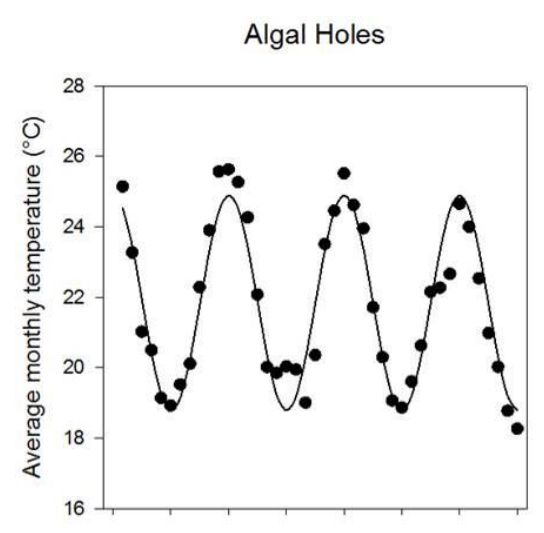

Malabar

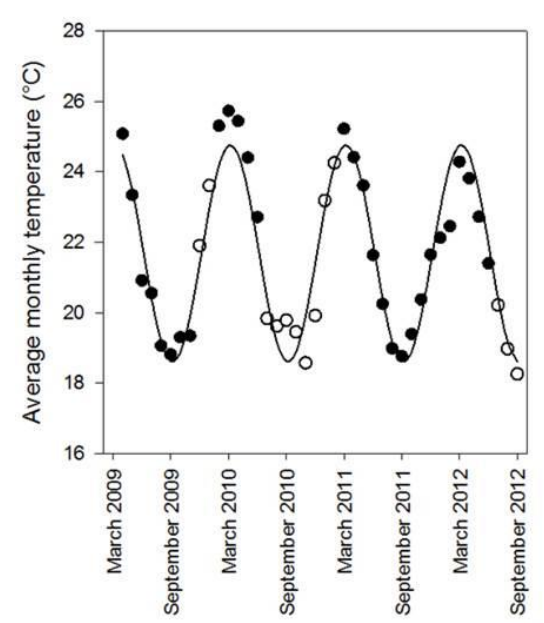

Comet's Hole

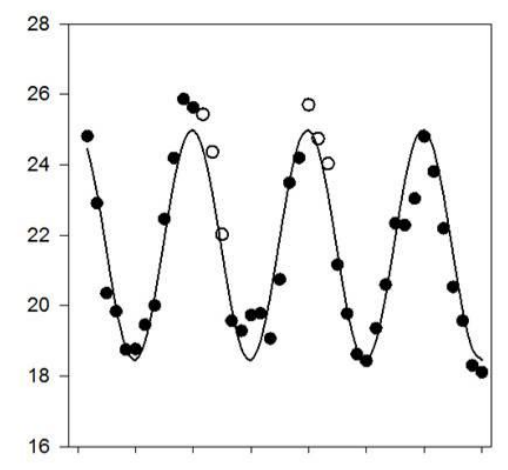

Ned's Beach

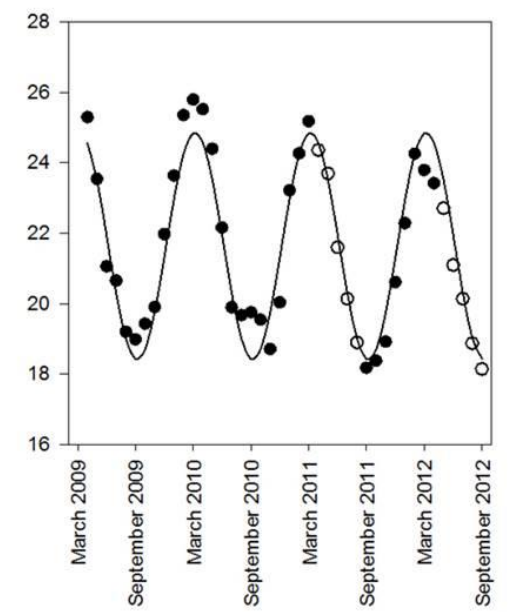

North Bay

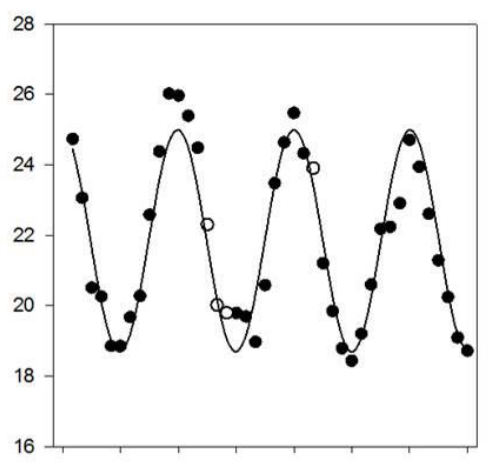

Sylph's Hole

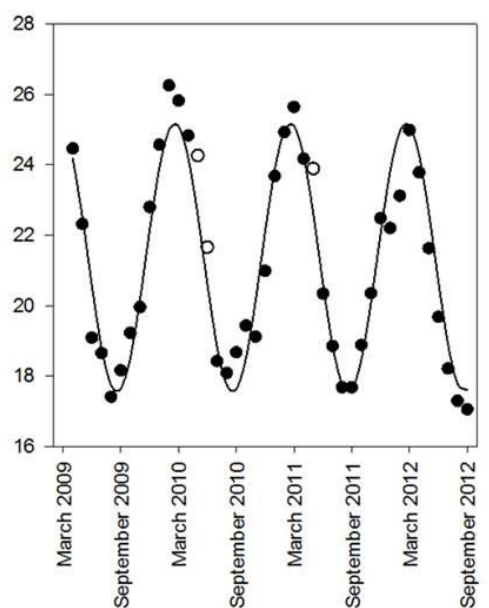

Sugarloaf West

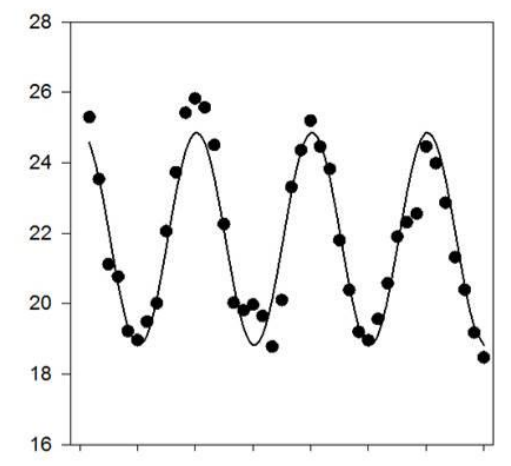

The Arch

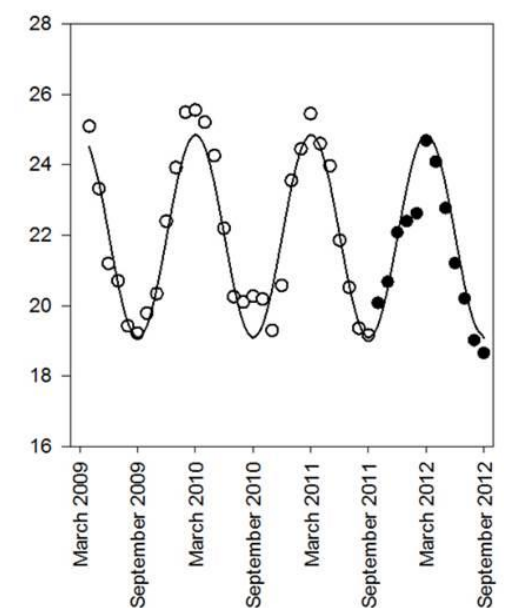

Figure 3.3 Thermal characteristics of eight Lord Howe Island reef sites

Average monthly temperatures obtained from data-logger measurements are shown in closed circles. Open circles show average monthly temperatures during logging gaps, estimated using correlation analysis against sites from which continuous measurements were available. Measurements from Algal Hole North, Comet's Hole, Malabar, Ned's Beach, North Bay, Sugarloaf West and Sylph's Hole were acquired from the Australian Institute of Marine Science (AIMS). Measurements from The Arch were obtained from a HOBO data logger, deployed from September 2011 to September 2012. Overlying fitted curves are optimized four-parameter sine functions. Note that extensive coral bleaching occurred during early 2010 (Harrison et al. 2011). 

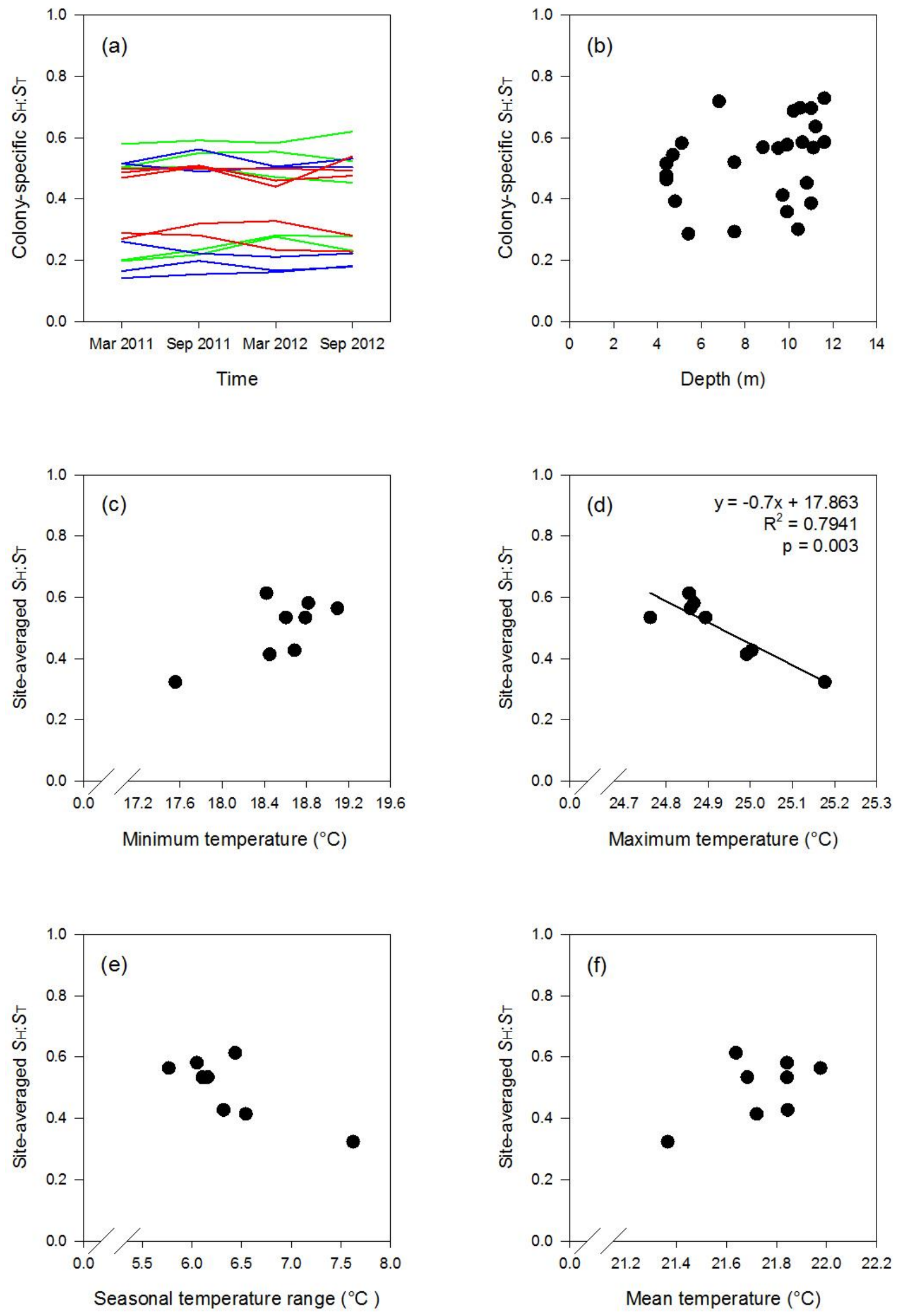


\section{Figure 3.4 (previous page) Niche partitioning of putative Symbiodinium hybrids}

The proportional abundance of genetically heterogeneous Symbiodinium cells $\left(S_{\mathrm{H}}: S_{\mathrm{T}}\right)$ was characterized by: (a) temporal stability within coral colonies over two summer-winter cycles (colonies from Comet's Hole are shown in red, North Bay in green and Sylph's Hole in blue); (b) no association between depth and colony-averaged $S_{\mathrm{H}}: S_{\mathrm{T}}$ at a steeply sloping reef-margin site (The Arch); and (c-f) relationships between site-averaged $S_{\mathrm{H}}: S_{\mathrm{T}}$ and four candidate thermal predictor variables extracted from the climate model. Of the model-fitted thermal minimum (c), thermal maximum (d), seasonal temperature range (e), and temperature mean (f), only the thermal maximum showed a significant (negative) correlation with $S_{\mathrm{H}}: S_{\mathrm{T}}$.

\subsection{Discussion}

This study explored the relationship between the physical environment and the abundance of putative Symbiodinium hybrids. The methods described in chapter 2 were expanded in order to measure the proportional abundance of putative hybrids in mixed Symbiodinium communities, facilitating a thorough investigation of their spatiotemporal distribution. Each coral colony hosted a specific proportion of putative Symbiodinium hybrids that remained stable over two summer-winter cycles. The data show an absence of competition or withincolony niche partitioning between genetically homogeneous and heterogeneous symbionts; however thermal niche-partitioning was evident between colonies hosting different proportional abundances of putative hybrid symbionts. Corals dominated by these symbionts were comparatively rare within the lagoon, but abundant at the reef margin near the transition zone between coral- and macroalgal-dominated benthic communities. While this may have resulted from fine-scale population structure (by geographic distance or physical resistance to connectivity), the significant correlation with maximum temperature strongly suggests a thermal basis for the observed distribution pattern.

\subsubsection{Method development}

A primary outcome of this study was the quantification of genetically heterogeneous Symbiodinium cells using bulk-cell qPCR, a development that circumvented the need to carry out single-cell analysis on each coral colony. rDNA markers (such as the ITS2) have been targeted in several quantitative assessments of mixed-clade infections (Ulstrup \& van Oppen 2003; Loram et al. 2007a; Mieog et al. 2007; Venn et al. 2008; Correa et al. 2009; LaJeunesse et al. 2009; Yamashita et al. 2010; Green et al. 2014). When properly calibrated, 
these assays can provide reliable estimates of symbiont relative abundance, despite the high variability in rDNA total copy-numbers often detected between Symbiodinium cells (Loram et al. 2007a; Mieog et al. 2007). In this study, the use of the ITS2 in determining symbiont community dynamics was validated by single-cell analysis in conjunction with a robust statistical method (model selection; Burnham \& Anderson 2002). Yet the conversion of bulkcell ITS2 ratios $\left(\mathrm{C}_{\mathrm{C} 100}: \mathrm{C}_{\text {TOTAL }}\right)$ to the proportional abundance of genetically heterogeneous Symbiodinium cells $\left(S_{\mathrm{H}}: S_{\mathrm{T}}\right)$ was supported by a small sample size and only a moderate correlation ( ix coral colonies; $\mathrm{R}^{2}=0.6$ ), representing an obvious weakness in this analysis. Furthermore, the identification of Symbiodinium ITS2 types was based on DGGE, an electrophoretic method that lacks sensitivity in detecting background symbiont taxa (Thornhill et al. 2006b; LaJeunesse et al. 2008). DGGE yielded a minimum $\mathrm{C}_{\mathrm{C} 100}: \mathrm{C}_{\text {TOTAL }}$ detection limit of approximately $15 \%$ for nested PCR (see chapter 2 ) and $\sim 20 \%$ for direct PCR amplification; therefore other cryptic symbiont types may have gone undetected. Another limitation of DGGE involves sequence co-migration. Though unlikely, other symbiont types may have been present and remained undetected. For example, the C103 sequence migrates to a similar position on the denaturing gradient as C109 (see Wicks et al. 2010, supplementary material). These limitations may eventually be overcome through the application of quantitative next-generation sequencing (NGS) to individual cells. Nonetheless, the bulk-cell qPCR method developed here showed an adequate ability to quantify ratios of genetically heterogeneous and homogeneous cells in the $P$. damicornis symbiont consortium.

\subsubsection{The spatiotemporal distribution of putative Symbiodinium hybrids}

Genetically heterogeneous and homogeneous Symbiodinium cells were co-dominant within many $P$. damicornis colonies, with $S_{\mathrm{H}}: S_{\mathrm{T}}$ values ranging from $15 \%$ to $82 \%$. This finding contrasts with several quantitative analyses of mixed-clade infections, in which the most common condition involves a single dominant type coexisting with one or a few cryptic/background populations (Ulstrup \& van Oppen 2003; Mieog et al. 2007; Correa et al. 2009; LaJeunesse et al. 2009; Yamashita et al. 2010; Byler et al. 2013; Cunning et al. 2013). For example, in five host species that hosted mixed-clade Symbiodinium assemblages, the abundance ratio of non-dominant to dominant symbionts ranged from $10^{-3}$ to $10^{-6}$ (Correa et al. 2009). Where symbiont co-dominance does exist, it generally involves either competitive 
displacement or within-colony niche-partitioning (Rowan \& Knowlton 1995; Rowan et al. 1997; van Oppen et al. 2001; Ulstrup \& van Oppen 2003; Chen et al. 2005; Ulstrup et al. 2006, 2007; Thornhill et al. 2006b; LaJeunesse et al. 2010b; Pettay et al. 2011). For example, Caribbean Montastraea (= Orbicella) annularis colonies can simultaneously host members of Symbiodinium clades A, B and C, with clade C dominating shaded sides, clade B dominating the light-exposed tips and clade A occupying intermediate irradiance-microhabitats (Rowan et al. 1997). In a conspicuous example of competition among mixed symbionts, a temporary outbreak of the opportunistic type $\mathrm{B} 1_{\text {Aiptasia }}$ (= Symbiodinium minutum) was visible as yellow patches on branches of Pocillopora spp. following a cold-water bleaching event in the Gulf of California (LaJeunesse et al. 2010b).While samples were not taken from all physical aspects of colonies in the present study, the relative abundance of genetically homogeneous and heterogeneous Symbiodinium cells was noticeably uniform across the topography of all colonies. Indeed, the $\sim 4 \%$ of total $S_{\mathrm{H}}: S_{\mathrm{T}}$ variation that was detected within colonies is likely to be an inflated estimate, since this residual variance component also encompasses pipetteand instrument-error. The co-dominant, uniform distribution pattern of coexisting Symbiodinium cells within colonies suggests that neither genotype shows opportunistic tendencies, and that symbiont niche-partitioning does not occur within P. damicornis colonies.

A lack of competition between genetically homogeneous and heterogeneous Symbiodinium cells is further indicated by the absence of dynamic symbiont shuffling. By contrast, competition between symbionts for host resources generally manifests in transient infections or temporal fluctuations in relative abundance (e.g. Thornhill et al. 2006; Jones et al. 2008; LaJeunesse et al. 2009, 2010). The temporal stability in $S_{\mathrm{H}}: S_{\mathrm{T}}$ observed here also suggests that the consortium dynamic is not affected by natural fluctuations in the physical environment, as evident in other corals and symbiotic anemones that host mixed Symbiodinium infections (Chen et al. 2005; Venn et al. 2008). Rather, it indicates that either symbiont shuffling is prompted by severe environmental disturbance, through selective symbiont expulsion/regeneration during bleaching (sensu Yamashita et al. 2010), or noncompetitive clonal genotypes establish during early ontogeny/gametogenesis (sensu PadillaGamiño et al. 2012) and are not subject to further modification. Several cases of bleachinginduced symbiont shuffling are reported from tagging and transplantation studies (Baker 2001; Toller et al. 2001b; Berkelmans \& van Oppen 2006; Thornhill et al. 2006b; Jones et al. 2008; LaJeunesse et al. 2009). Yet in studies employing long-term, follow-up monitoring, the 
eventual return to dominance of a homologous type is usually evident (Thornhill et al. 2006b; Jones et al. 2008; Sampayo et al. 2008; LaJeunesse et al. 2009). While no observable bleaching occurred during this study, a severe bleaching episode affected the LHI lagoon approximately one year prior to the first round of sampling (Harrison et al. 2011). Therefore, if bleaching did cause symbiont shuffling in adult colonies, at least some readjustment in $S_{\mathrm{H}}: S_{\mathrm{T}}$ would likely have been observed during this study. The pronounced temporal conservation in $S_{\mathrm{H}}: S_{\mathrm{T}}$ within colonies despite previous bleaching suggests that, although thermal stress is important in determining symbiont ratios (see next section), this most likely occurs through early ontogenetic flexibility or natural selection acting on juvenile and/or larval stages.

\subsubsection{Niche partitioning of putative Symbiodinium hybrids and progenitors}

The summer thermal maximum emerged as a significant predictor of $S_{\mathrm{H}}: S_{\mathrm{T}}$, while the thermal minimum, average temperature, seasonal temperature variability, and depth (a proxy for irradiance) did not have any detectable influence. Colonies dominated by genetically heterogeneous Symbiodinium cells were more common at the reef-margin sites than in the lagoon, where reduced circulation stimulates more pronounced thermal maxima (I. Kerr, LHIMPA, pers. comm.). A degree of niche partitioning between genetically homogeneous and heterogeneous symbionts therefore appears to be supported by differences in thermal tolerance. Symbiont niche partitioning is evident both within and between colonies of many coral species, with temperature and irradiance identified as key drivers (Rowan \& Knowlton 1995; Rowan et al. 1997; van Oppen et al. 2001; Ulstrup \& van Oppen 2003; Iglesias-Prieto et al. 2004; Ulstrup et al. 2006; Berkelmans \& van Oppen 2006; Sampayo et al. 2007). This pattern is attributable to the evolution of physiological adaptations that enable survival in a particular light or thermal habitat, and the out-competition of extrinsic or generalist types (Ulstrup \& van Oppen 2003). The observed thermal niche partitioning between genetically homogeneous and heterogeneous Symbiodinium cells therefore supports their delineation as separate genetic and ecological entities, lending further credibility to the hypothesis of symbiont hybridization.

Hybrids are often rapidly out-competed by their progenitors when in the parental habitat (e.g. Brasier 2001). Reduced hybrid fitness is particularly conspicuous in haploids, since detrimental effects arising from the segregation of co-adapted gene complexes during 
recombination (hybrid breakdown) cannot be compensated for by heterosis (i.e. 'hybrid vigour' arising from increased heterozygosity in early generation hybrids; Charlesworth \& Charlesworth 1987). The reduced abundance of genetically heterogeneous Symbiodinium cells within the LHI lagoon is consistent with reduced hybrid fitness in this habitat; yet their ecological prosperity at the transitional reef margin sites remains an intriguing puzzle. The diversification of transgressive hybrids into this 'extreme' habitat presents a plausible explanation that warrants further investigation. In this context, a large-scale assessment of hybrid abundance along a latitudinal gradient spanning both tropical and sub-tropical reefs would provide a valuable contribution to this research.

\subsubsection{Conclusion}

The application of bulk-cell qPCR enabled the rapid estimation of the proportional abundance of genetically heterogeneous Symbiodinium cells in P. damicornis colonies. This facilitated a comprehensive assessment of the spatiotemporal distribution of these putative Symbiodinium hybrids. Negligible variability in symbiont abundance ratios within colonies suggested an early ontogenetic establishment of a few non-competitive clonal genotypes. While homogeneous and heterogeneous Symbiodinium cells were relatively co-dominant within many colonies, a degree of thermal niche-partitioning was evident between $P$. damicornis colonies hosting different symbiont abundance ratios. Specifically, those dominated by heterogeneous symbionts were common at the reef margin, and comparatively rare at the inner lagoon sites that experience more pronounced thermal peaks. Ecological differentiation between genetically homogeneous and heterogeneous Symbiodinium cells lends further credibility to the occurrence of hybridization, and may indicate the diversification of hybrids into the 'extreme' habitat at the margin of coral- and macroalgal-dominated benthic assemblages. 


\section{Chapter 4: The form and function of putative Symbiodinium hybrids}

\subsection{Introduction}

Natural hybridization has played defining roles in the evolution of many species. New genetic variation is created through the migration of genetic material between lineages, via the repeated backcrossing of hybrids to parental lineages (introgression; Arnold 2007). Additionally, the substantial genetic variation or 'macro-mutation' that is created during outcrossing has led to several cases of hybrid speciation, and even adaptive radiations in plant and animal taxa (Rieseberg 1997; Bell \& Travis 2005; Grant et al. 2005; Mallet 2007). However, the potential for hybridization to contribute to adaptation depends on the fitness of $F_{1}$ hybrids and later-generation hybrid and backcross classes (i.e. $F_{2}, F_{3}$, etc.; Barton 2001). In particular, gene flow will not overcome barriers of reproductive isolation if the mean fitness of any of the intermediate backcross classes is insurmountably low (Barton \& Bengtsson 1986; Ingvarsson \& Whitlock 2000; see chapter 2, Figure 2.1). If considerable time has passed since divergence, the vast majority of the innumerable possible hybrid and backcross recombinants will be inviable or unfit, representing biological 'dead ends' with little or no evolutionary significance (Barton 2001). This poor relative fitness arises from epistatic 'hybrid breakdown', the segregation of co-adapted gene complexes or the creation of maladapted gene combinations (Demuth \& Wade 2005). Early-generation hybrid breakdown is particularly evident in haploids, whose unpaired chromosomes cannot compensate for the detrimental effects of recombination via heterosis (i.e. 'hybrid vigour' arising from increased heterozygosity in diploid or polyploid hybrids; Charlesworth \& Charlesworth 1987). In extreme cases, post-zygotic lethality can result from the failure to proceed from meiotic to mitotic cell division (e.g. Brosnahan et al. 2010). However, hybrid performance and fitness may occasionally equal or even exceed those of either parent taxon, where conditions are experienced between or outside their environmental optima (Anderson \& Stebbins 1954; Arnold \& Hodges 1995; Rieseberg et al. 2003; Arnold \& Martin 2010). Under such conditions, the rate of gene-flow increases and hybridization can stimulate rapid adaptation (Ingvarsson \& Whitlock 2000). 
Genetically heterogeneous Symbiodinium cells can form an abundant component of the symbiont consortium of $P$. damicornis (> $80 \%$; see chapters 2 and 3 ). The fitness of at least one heterogeneous clone therefore appears sufficient to overcome the selective pressures imposed by the abiotic environment and the coral host. Indeed, the considerable variation in ITS2 ratios and total copy numbers (see chapter 2) suggest that genetically heterogeneous symbiont populations may be comprised of several fit clonal genotypes. If frequent hybridization underlies this genetic variation, the fitness of each of the many different hybrid recombinants is likely to be correspondingly variable (Barton 2001). Addressing the question of hybrid fitness may be simplified by determining the average fitness of each discrete hybrid class. However, even if the occurrence of hybridization between Symbiodinium C100 and C109 can be confirmed, the frequency distribution of ITS2 ratios in a population (e.g. chapter 2, Figure 2.4) may not provide sufficient resolution to distinguish between classes. This is because variation in ITS2 ratios may arise from multiple processes, including concerted evolution acting on ancestral polymorphisms, differential rDNA inheritance (arising from dissimilar copy-numbers in parent taxa; e.g. Brosnahan et al. 2010), the occurrence of backcrossing, and even the rapid homogenization of intra-genomic variation following hybridization (Wendel et al. 1995). The analysis of multiple diagnostic loci is therefore required to establish the incidence of hybridization and backcrossing, and evaluate the fitness of each hybrid class (Nason \& Ellstrand 1993; Epifanio \& Phillipp 1997; Fitzpatrick 2012). Though technically possible, this is currently a challenging task for individual cells (Handyside et al. 2004).

The resource limitations of this project preclude the unambiguous confirmation of genetically heterogeneous Symbiodinium cells as hybrid and backcross genotypes. However, it is currently possible to broadly classify individuals into putative-hybrid or non-hybrid classes using mixture-model clustering (see chapter 2), enabling an initial comparative assessment of their performance and fitness relative to the putative progenitor Symbiodinium C100. This chapter aims to establish whether genetically homogeneous and heterogeneous Symbiodinium cells show overall differences in morphology, physiology and performance, and if so, whether the relative abundance of each type in the symbiont consortium influences the functioning of the coral symbiosis. Assessments of coral- and symbiont physiology were made at both ambient and artificially-elevated seawater temperatures $\left(25\right.$ and $29^{\circ} \mathrm{C}$, respectively) to explore the influence of thermal stress on the performance of each symbiont cell-type. The hypotheses tested were that: (1) genetically homogeneous and heterogeneous 
Symbiodinium cells show differences in morphology and photo-physiology; (2) corals hosting different proportions of genetically homogeneous and heterogeneous symbionts show corresponding physiological differences; and (3) elevated temperatures exacerbate the functional disparities between genetically homogeneous and heterogeneous symbionts, with corresponding effects on the host's physiological performance.

\subsection{Methods}

\subsubsection{Study location and species}

The corals investigated in this study were Pocillopora damicornis colonies inhabiting the reef at North Bay, LHI $(-31.521,159.047)$. At this location, P. damicornis can simultaneously host three genetically distinct Symbiodinium ITS2 types, C100, C109, and symbiont cells featuring a heterogeneous mixture of C100 and C109 sequences (putative hybrids; see chapters 2 and 3). Genetically heterogeneous Symbiodinium cells are generally less common in the LHI lagoon (including North Bay), than at the reef margin. This thermal nichepartitioning pattern appears to be driven by temperature stress, since the site-averaged proportional abundance of genetically heterogeneous Symbiodinium cells is negatively correlated with the summer thermal maximum (see chapter 3). The poorly-flushed innerlagoon sites experience particularly pronounced thermal maxima, with model-fitted values of $25.003{ }^{\circ} \mathrm{C}, 25.177{ }^{\circ} \mathrm{C}$ and $24.991{ }^{\circ} \mathrm{C}$ for North Bay, Sylph's Hole and Comet's Hole, respectively. However, the actual temperature can occasionally exceed these values, with the water temperature at Comet's Hole reaching $28.16{ }^{\circ} \mathrm{C}$ during January 2010 (see chapter 3).

\subsubsection{Coral collection and acclimation}

Coral collection, acclimation and oxygen flux analysis was carried out during March 2012. One branch was collected from each of 15 P. damicornis colonies by divers using diagonal pliers. Each branch was then divided into two small fragments (surface area approximately 6 $\mathrm{cm}^{2}$ ), which were then attached to stainless steel bases with marine epoxy putty to maintain an upright orientation (Knead It Aqua; Selleys). One fragment from each colony was assigned to a treatment group and the other to a control group. Fragments were placed in 21 clear plastic tanks ( 5 fragments per tank; 3 tanks per treatment; Figure 4.1) and supplied with 
flowing seawater at rate of $300 \mathrm{ml} \mathrm{min}^{-1}$, drawn from a 2001 reservoir. This reservoir was maintained at $25^{\circ} \mathrm{C}$ using submersible heaters and an aquarium chiller unit (Hailea HC500A), and was fed from a larger reservoir, into which fresh seawater was continuously pumped from the LHI lagoon. All fragments were initially acclimated for $5 \mathrm{~d}$ at $25^{\circ} \mathrm{C}$, after which the temperature in the three tanks housing the treatment-group fragments was raised to $29{ }^{\circ} \mathrm{C}$ over the course of $4 \mathrm{~h}$ (constant ramp rate of $1^{\circ} \mathrm{C} \mathrm{h}^{-1}$ ). This was achieved by switching the water supply of the treatment tanks to a second temperature-controlled 2001 tank (the control tanks remained at $25^{\circ} \mathrm{C}$ ). All fragments were then left at their respective temperatures for a further $4 \mathrm{~d}$ prior to oxygen flux analysis, sample preservation (immediate freezing) and laboratory processing. 


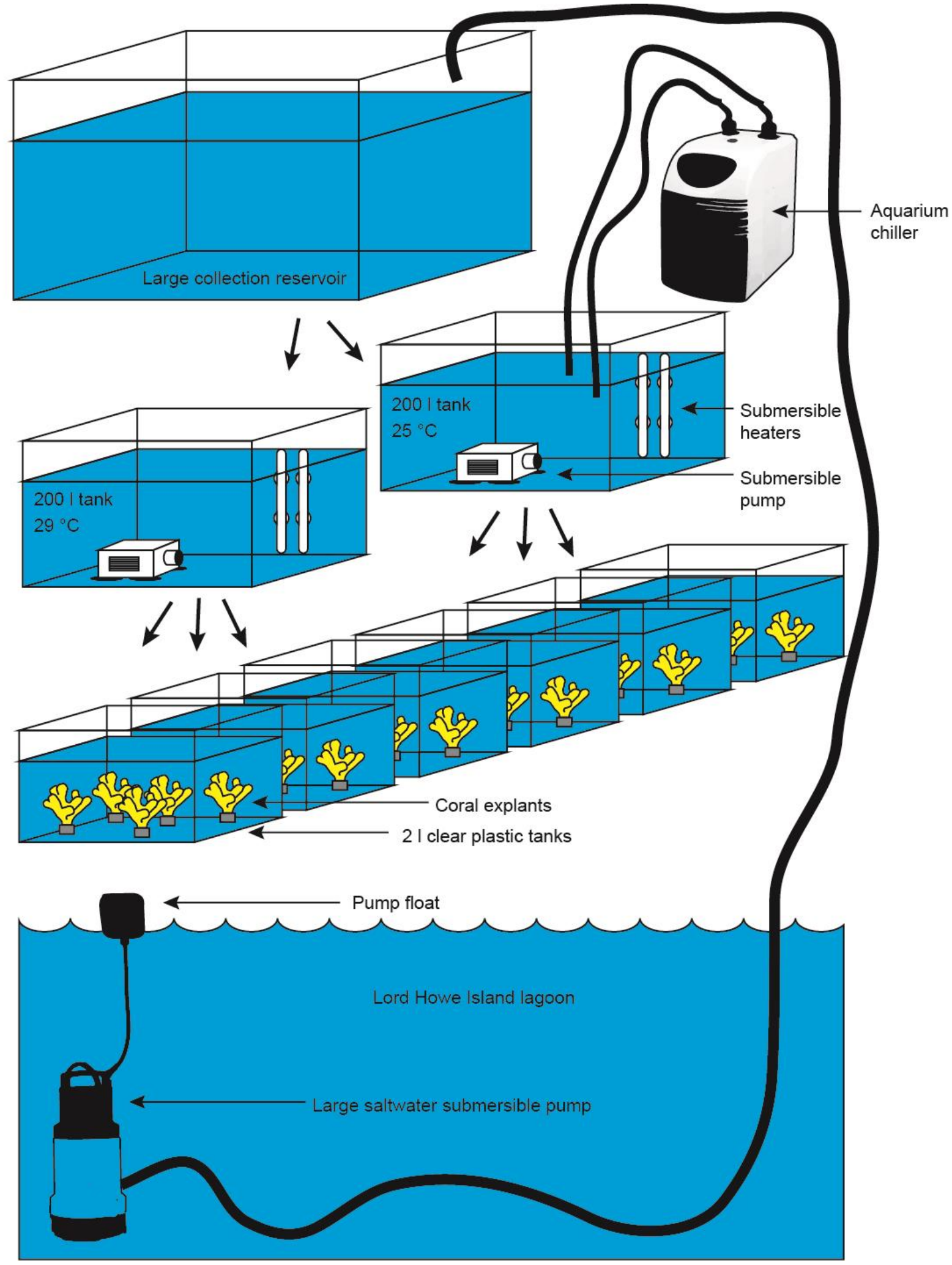

Figure 4.1 Thermal acclimation of coral fragments prior to oxygen flux analysis

Seawater was supplied from the LHI lagoon into a large collection reservoir using a submersible pump (Leader Ecosub). The large reservoir fed into two 2001 tanks, in which temperatures were maintained using submersible aquarium heaters, circulation pumps and in the case of the $25{ }^{\circ} \mathrm{C}$ tank, an aquarium chiller. Water was then fed from the temperature-controlled tanks into 21 clear plastic tanks housing coral fragments (five per tank), at a constant rate of $300 \mathrm{ml} \mathrm{min}^{-1}$. 


\subsubsection{Analysis of oxygen fluxes}

For the analysis of photosynthetic and respiratory oxygen fluxes, each coral fragment was transferred to an airtight $15 \mathrm{ml}$ glass chamber filled with $1 \mu \mathrm{m}$ filtered seawater (FSW; Figure 4.2). The volume of the FSW displaced was measured using an analytical balance, and the concentration of dissolved oxygen in the chamber was calculated using coefficients derived from tables of oxygen solubility at $35 \%$ salinity $\left(211.3\right.$ and $194.6 \mu \mathrm{mol} \mathrm{O} \mathrm{O}^{-1}$ for $25{ }^{\circ} \mathrm{C}$ and $29{ }^{\circ} \mathrm{C}$, respectively). A pre-calibrated fiber-optic oxygen electrode (FIBOX 3; PreSens $\mathrm{GmbH}$ ) and temperature sensor were inserted through the sealed openings of the chamber, and a visual check was carried out to ensure that no air bubbles were present. The chamber was then submerged in a temperature-controlled water bath containing a submersible aquarium heater, a temperature probe and an aquarium pump (to ensure even temperature distribution). FSW in the chambers was mixed by a micro-spin bar controlled by a magnetic stirrer, and temperatures were maintained within $0.2{ }^{\circ} \mathrm{C}$ of target values $\left(25^{\circ} \mathrm{C}\right.$ and $29{ }^{\circ} \mathrm{C}$ for control and treatment fragments, respectively) by manually controlling the submersible heater unit. Following a 10 min dark acclimation period, fragments were exposed to eight consecutive irradiance levels $\left(0,10,20,40,80,160,320\right.$ and $700 \mu \mathrm{mol}$ photons $\mathrm{m}^{-2} \mathrm{~s}^{-1}$; measured using a LI-COR LI-190 underwater quantum sensor). Light was provided by a 12V halogen lamp, directed through a series of $50 \%$ transmittance neutral density filters. Fragments were acclimated to each light level for $5 \mathrm{~min}$, before the dissolved oxygen in the chamber was measured at $5 \mathrm{~s}$ intervals for at least $5 \mathrm{~min}$, until a linear rate of oxygen evolution was observable. Net oxygen evolution rates at each light level were calculated by fitting a linear regression line. Oxygen electrodes were subjected to two-point dissolved oxygen calibration prior to each run. FSW was treated with sodium sulphite $\left(1 \mathrm{gl}^{-1} \mathrm{Na}_{2} \mathrm{SO}_{3}\right)$ for the $0 \%$ dissolved oxygen calibration, and oxygenated for 30 min using an aquarium air pump and air-stone for the $100 \%$ dissolved oxygen calibration. The oxygen saturation in the chamber was maintained between 50 and 100\% (Davies 1984). Following oxygen flux analysis, fragments were frozen at $-20{ }^{\circ} \mathrm{C}$ for subsequent laboratory analysis. 


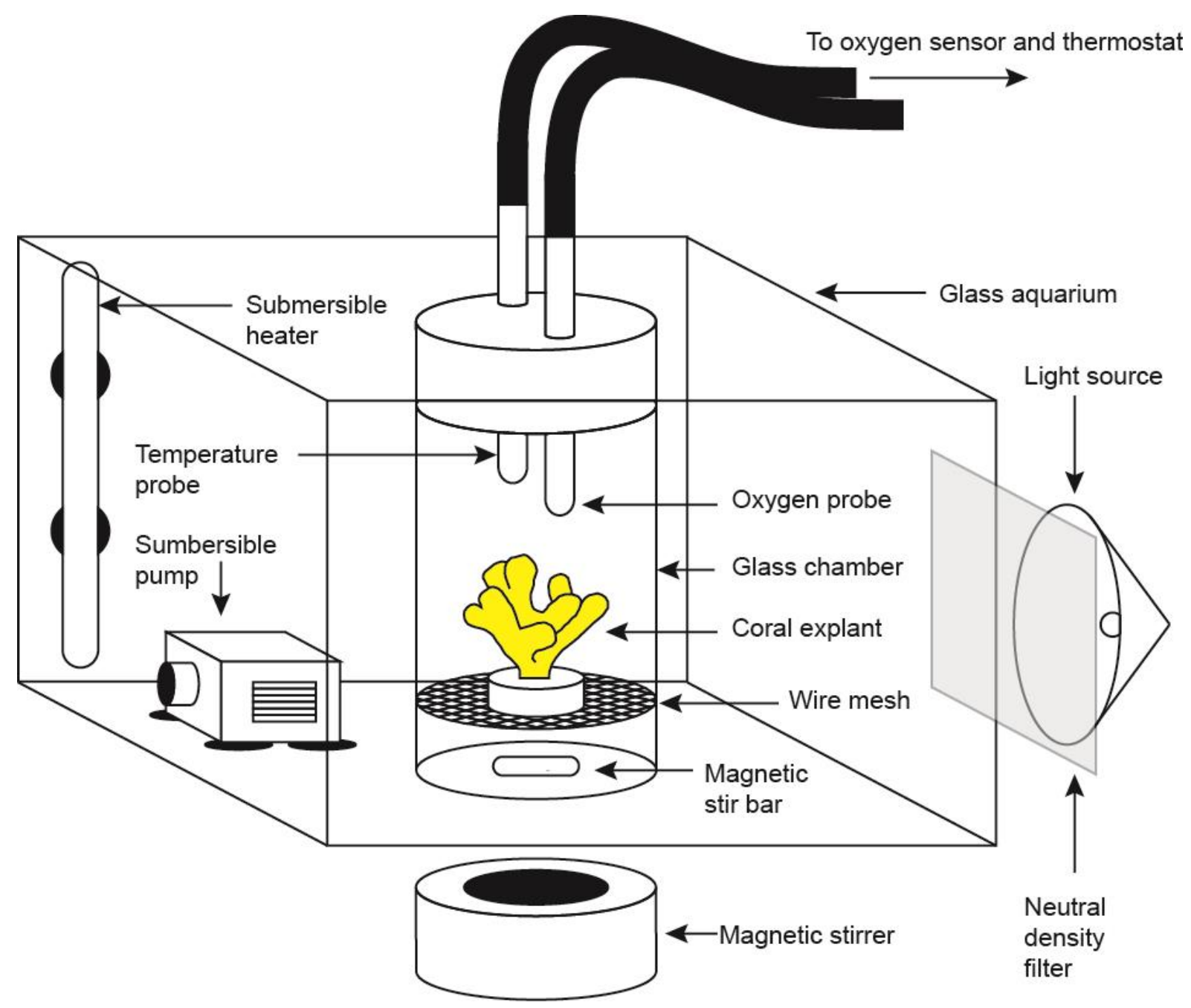

Figure 4.2 Experimental setup for measuring coral oxygen fluxes

Coral fragments were individually placed in a sealed $15 \mathrm{ml}$ glass chamber, and maintained at a constant temperature of 25 or $29{ }^{\circ} \mathrm{C}$ by manually controlling a submersible heater. Water inside the chamber was mixed by a stir bar, controlled by a magnetic stirrer situated beneath the aquarium. Oxygen fluxes were measured at each of eight light levels ranging from 0 to $700 \mu$ mol photons $\mathrm{m}^{-2} \mathrm{~s}^{-1}$, generated by placing a series of neutral density filters between the light source and the aquarium.

\subsubsection{Assessment of morphology and physiology}

Frozen coral fragments were defrosted and coral tissue was removed from the underlying skeleton with a stream of $50 \mathrm{mM}$ phosphate buffer ( $1 \mathrm{mM}$ EDTA; $\mathrm{pH}=7.8)$, delivered at high velocity through a compressed-air-generated spray nozzle. The coral skeleton was dried at $70{ }^{\circ} \mathrm{C}$ for $24 \mathrm{~h}$ and the surface area calculated using the wax method (Stimson \& Kinzie 1991). The total homogenate volume was measured using a pipette, and five $1.5 \mathrm{ml}$ aliquots 
were taken for cell density and symbiont size measurements (one aliquot), DNA extraction (one aliquot), and measurements of host protein biomass and symbiont chlorophyll composition (three aliquots). Symbiont densities were estimated using a haemocytometer (Neubauer Brightline; eight replicate grids for each sample). The average symbiont size was assessed by measuring the diameter of 50 cells from each coral fragment, using a compound microscope fitted with an eyepiece graticule and a 100x objective oil immersion lens. The three protein/chlorophyll aliquots were centrifuged at $16,100 \mathrm{x}$ g for $5 \mathrm{~min}$ to separate host and algal fractions. For host protein biomass estimation, $10 \mu \mathrm{l}$ of supernatant were added to each of three micro-plate wells, each containing $200 \mu 1$ of Bradford reagent (Sigma-Aldrich). Sample absorbance was measured at $595 \mathrm{~nm}$, and the content of host-derived soluble protein was estimated by comparing absorbance values to a standard curve obtained using BSA protein standards. The soluble host protein biomass from each coral fragment was normalized to the skeletal surface area, and expressed in $\mathrm{mg} \mathrm{cm}^{-2}$. To extract and measure chlorophyll from within the Symbiodinium cells, algal pellets were re-suspended in $222 \mu 190 \%$ acetone and incubated in a light-proof container for $24 \mathrm{~h}$ at $4{ }^{\circ} \mathrm{C}$. Chlorophyll extracts were then centrifuged at $16,100 \times \mathrm{g}$ to pellet cellular debris and chlorophyllases. The supernatant (200 $\mu 1)$ was transferred to a UV-proof micro-plate (Griener) and absorbance values were measured at 630, 665 and $750 \mathrm{~nm}$. The concentration of chlorophylls $a$ and $c_{2}$ were calculated using the equations of Jeffrey \& Humphrey (1975) with the coefficients for dinophytes given by Ritchie (2006). Chlorophyll content was expressed as pg cell ${ }^{-1}$.

\subsubsection{DNA extraction}

For bulk-cell PCR and qPCR analysis of Symbiodinium ITS2 sequences, a $1.5 \mathrm{ml}$ homogenate sample was centrifuged at $16,100 \mathrm{x}$ g for $5 \mathrm{~min}$, the supernatant was discarded, and $200 \mu \mathrm{l}$ DMSO preservation buffer (20\% DMSO, 250 mM EDTA, NaCl saturated, pH 8.0; Seutin et al. 1991) were added. The pellet was re-suspended by milling without beads for 3 min at 50 Hz. Following a $7 \mathrm{~d}$ incubation period at $-20^{\circ} \mathrm{C}$, the settled Symbiodinium cells were resuspended using a vortex and $10 \mu \mathrm{l}$ were taken for DNA extraction. Acid-washed glass beads were added (50 mg; 710-1180 $\mu \mathrm{m}$; Sigma-Aldrich) and the sample was milled at $50 \mathrm{~Hz}$ for 3 min (Qiagen TissueLyser LT; Qiagen) to disrupt the cell-walls and enable the release of nucleic acids. Following a $10 \mathrm{~min}$ incubation period at $4{ }^{\circ} \mathrm{C}, 90 \mu \mathrm{l}$ of de-ionised water were added. The sample was then vortexed and centrifuged for $10 \mathrm{~min}$ at $16,100 \mathrm{x}$ g to pellet the 
cellular debris. The DNA-enriched supernatant $(50 \mu \mathrm{l})$ was transferred to a new microcentrifuge tube with an equal volume of 2-propanol to precipitate the nucleic acids, and centrifuged for $10 \mathrm{~min}$ at $16,100 \mathrm{x} \mathrm{g}$ to collect the DNA pellet. The supernatant was discarded and $200 \mu \mathrm{l}$ wash buffer (70\% v/v ethanol) were added. The sample was vortexed and returned to the centrifuge for a further $10 \mathrm{~min}$ at $16,100 \mathrm{x} \mathrm{g}$. The supernatant was carefully removed with a pipette and the DNA pellet was dried under a laminar flow hood for $30 \mathrm{~min}$. Elution buffer (10 mM Tris-HCl; $0.1 \mathrm{mM}$ EDTA; $\mathrm{pH}=8.0)$ was added to a final volume of $50 \mu \mathrm{l}$. Following a 10 min re-hydration period, the DNA was re-suspended by milling without beads for $1 \mathrm{~min}$ at $30 \mathrm{~Hz}$.

\subsection{6. $P C R$ and denaturing gradient gel electrophoresis}

Denaturing gradient gel electrophoresis (DGGE) was carried out on all samples to establish whether Symbiodinium types other than C100 and C109 were present in the selected colonies. PCR amplification for DGGE was carried out with the primers ITSintfor2 and ITS2CLAMP (LaJeunesse 2002). Thermal cycling involved an initial denaturation step of 3 min at $95{ }^{\circ} \mathrm{C}$ followed by 40 cycles of 15 seconds at $95{ }^{\circ} \mathrm{C}, 15$ seconds at $56{ }^{\circ} \mathrm{C}$ and 10 seconds at $72{ }^{\circ} \mathrm{C}$ (carried out on an Applied Biosystems Veriti thermo-cycler; Life Technologies). Each reaction contained $4 \mu \mathrm{l}$ of DNA template solution, 1x MyTaq PCR reaction mix (Bioline), 10 pmol each primer, $10 \mu \mathrm{g}$ bovine serum albumin (BSA; Sigma) and deionised sterile water to a total volume of $20 \mu \mathrm{l}$. A template-free control was included with each run. To ensure the final product was within the intended size range of 300-350 bp, and that amplification did not occur in the template-free control reaction, $5 \mu$ of the final PCR product were electrophoresed on a $1.5 \%$ agarose gel containing 1x SYBR safe nucleic acid stain (Life Technologies) alongside a DNA standard (Hyperladder II; Bioline). Agarose gels were viewed and imaged on a blue light trans-illuminator (Safe Imager; Invitrogen).The remaining PCR product was loaded on a $200 \times 200 \times 1 \mathrm{~mm}, 8 \%$ denaturing polyacrylamide gel (25-50\% urea/formamide denaturant gradient), and run in $1 \mathrm{x} \mathrm{TAE}$ at $150 \mathrm{~V}$ for $7 \mathrm{~h}$ at $60{ }^{\circ} \mathrm{C}$ (DCode system; BioRad) alongside known ITS2 sequences of Symbiodinium C100 and C109 (PCRamplified from plasmid DNA). Following electrophoresis, denaturing gels were stained with ethidium bromide and viewed on a UV trans-illuminator (FirstLight UVP), and the presence or absence of each ITS2 sequence type was scored. 


\subsubsection{Quantitative PCR}

The bulk-cell qPCR assay developed in chapter 3 was used to establish the relative proportion of putative Symbiodinium hybrids within the symbiont consortium of each coral fragment $\left(S_{\mathrm{H}}: S_{\mathrm{T}}\right)$. Each qPCR reaction contained $4 \mu \mathrm{l}$ DNA template, 1x TaqMan Universal Mastermix II (Life Technologies), 18 pmol each primer (CInnerFor and CInnerRev; see chapter 2 for

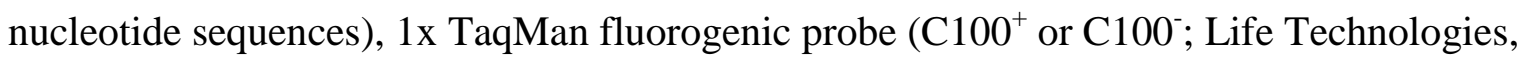
see chapter 2 for nucleotide sequences), $10 \mu \mathrm{g}$ BSA (Sigma-Aldrich) and deionised sterile water to a total volume of $20 \mu \mathrm{l}$. Thermal cycling involved an initial $10 \mathrm{~min}, 95^{\circ} \mathrm{C}$ denaturation step followed by 40 cycles of 15 seconds at $95{ }^{\circ} \mathrm{C}$ and $1 \mathrm{~min}$ at $60{ }^{\circ} \mathrm{C}$ (Applied Biosystems StepOne qPCR instrument; Life Technologies). DNA template solutions generated from bulk-cell Symbiodinium samples were run in duplicate with a template-free control reaction included for each probe type. $C_{t}$ values were determined as the cycle at which the change in fluorescence was significantly different to the background level $\left(\Delta R_{n}=\right.$ 0.05; obtained using the instrument's built-in algorithm). Reactions featuring $\mathrm{C}_{\mathrm{t}}$ values below the intercepts of the previously-generated standard curves $\left(36.873\right.$ and 37.119 for $\mathrm{C} 100^{+}$and $\mathrm{C} 100^{-}$assays, respectively; see Appendix $\left.B\right)$ and sufficiently low standard deviations $(<0.5)$ were included in the analysis. The total number of ITS 2 copies in each sample $\left(\mathrm{C}_{\mathrm{TOTAL}}\right)$ was estimated as the sum of the C100 copy number and the number of Symbiodinium clade C ITS2 sequences other than type $\mathrm{C} 100\left(\mathrm{C}_{\mathrm{C} 100}\right.$ and $\mathrm{C}_{\mathrm{C} 100}{ }^{-}$, respectively). The proportion of total Symbiodinium clade C sequences that were of type C100 was expressed as the bulk-cell $\mathrm{C}_{\mathrm{C} 100}$ : $\mathrm{C}_{\mathrm{TOTAL}}$ ratio. This ratio were subsequently converted to the proportional abundance of genetically heterogeneous cells $\left(S_{\mathrm{H}}: S_{\mathrm{T}}\right)$ using the polynomial equation developed in chapter 3 $\left(\mathrm{y}=-3.4484 \mathrm{x}^{2}+3.3604 \mathrm{x}\right.$; where $\mathrm{y}=S_{\mathrm{H}}: S_{\mathrm{T}}$ and $\left.\mathrm{x}=\mathrm{C}_{\mathrm{C} 100}: \mathrm{C}_{\mathrm{TOTAL}}\right)$.

\subsubsection{Statistical analysis}

For assessments of photosynthetic performance, a three-parameter hyperbolic tangent function of the form

$$
y=a\left(\frac{1-e^{\frac{-x}{b}}}{1-e^{\frac{-x}{b}}}\right)+c
$$


was fitted to the bivariate photosynthesis-irradiance data from each coral fragment (Chalker et al. 1983), using the least-squares curve fitter function in Sigmaplot v11.0 (Systat). The parameters $a, b$ and $c$ correspond to $P_{G R O S S}, I_{K}$ and $R$, representing the maximum gross rate of photosynthesis $\left(\mu \mathrm{mol} \mathrm{O} \mathrm{h}^{-1}\right)$, the $50 \%$ saturation irradiance $\left(\mu \mathrm{mol}\right.$ photons $\left.\mathrm{m}^{-2} \mathrm{~s}^{-1}\right)$, and the dark respiration rate $\left(\mu \mathrm{mol} \mathrm{O} \mathrm{O}^{-1}\right)$, respectively (Figure 4.3). Following parameter estimation, $P_{G R O S S}$ and $R$ were normalized to host protein biomass, and expressed in $\mathrm{nmol} \mathrm{O}_{2} \mathrm{mg}^{-1}$ protein $\mathrm{h}^{-1}$. The maximum gross photosynthesis to respiration ratio $(P: R)$, the light-harvesting efficiency $(\alpha)$ and the compensation irradiance $\left(I_{C}\right)$ were calculated using the formulae of Chalker et al. (1983):

$$
\begin{aligned}
& P: R=\frac{P_{\text {GROSS }}}{R} \\
& \alpha=\frac{P_{\text {GROSS }}}{I_{K}} \\
& I_{C}=I_{K} \tanh ^{-1} \frac{-R}{P_{\text {GROSS }}}
\end{aligned}
$$

Equation 4.4

Parametric multivariate analysis of covariance (MANCOVA) was used to compare the effects of temperature and the proportional abundance of genetically heterogeneous symbionts on the suite of measured photo-physiological response variables $\left(P_{G R O S S}, R, P: R\right.$, $\alpha, I_{C}$, and $I_{K}$ ) and morphological and physiological attributes (host protein biomass, symbiont density and size, and chlorophyll $a$ and $c_{2}$ content). Significant parameter estimates were then combined to generate an integrated photosynthesis-irradiance model with $S_{\mathrm{H}}: S_{\mathrm{T}}$ and temperature as explanatory variables. The average diameter and chlorophyll content of each symbiont cell type (i.e. genetically heterogeneous cells and homogeneous C100 cells) were estimated by extrapolating from the fitted regression lines (assuming hypothetical colonies with $S_{\mathrm{H}}: S_{\mathrm{T}}$ ratios of 0 and 1 ). In all cases, normality and variance homoscedasticity were assessed using Q-Q plots and Box's and Levene's tests of variance equality. All statistical analyses were carried out using SPSS Statistics v20 (IBM). 


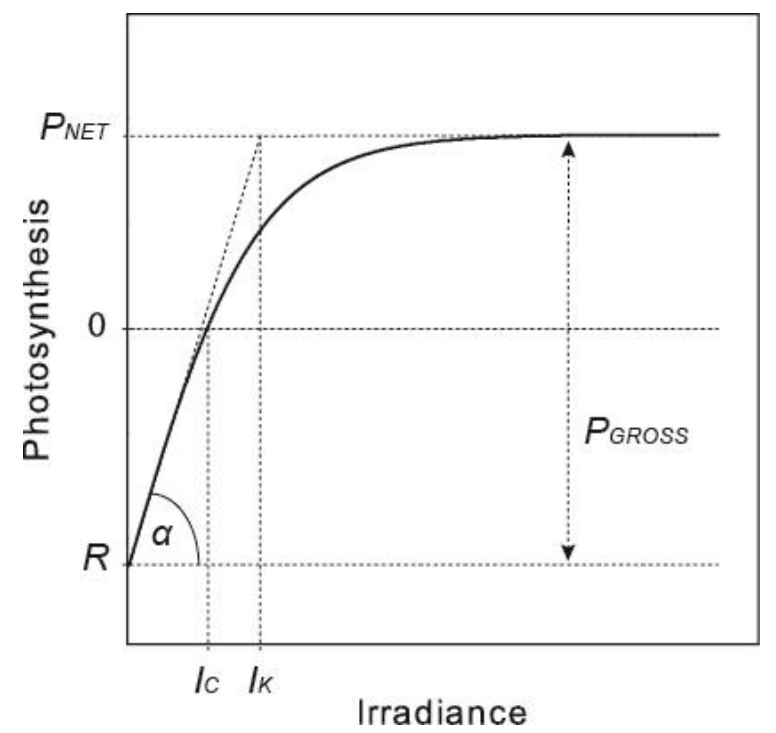

Figure 4.3 Properties of the photosynthesis-irradiance (PI) curve

The maximum net photosynthetic rate $\left(P_{N E T}\right)$ is the observed oxygen evolution at maximum irradiance. Respiration $(R)$ represents the total oxygen evolution in the dark, expressed as a negative value. The maximum gross photosynthetic rate $\left(P_{\text {GROSS }}\right)$ is the total photosynthetic oxygen evolution at maximum irradiance. The compensation irradiance $\left(I_{C}\right)$ is the irradiance at which no net oxygen evolution occurs $\left(P: R=1 ; P_{N E T}=0\right)$. The sub saturating irradiance $\left(I_{K}\right)$ is an indicator of photoacclimation, calculated as the theoretical point where the initial linear section of the curve intersects $P_{N E T}$. The light harvesting efficiency ( $\alpha$; also known as the light utilization coefficient) represents the effectiveness of photosynthesis at low light, and is calculated as the angle between the line $y=0$ and the initial linear section of the PI curve. Diagram adapted from Chalker et al. (1983).

\subsection{Results}

All 30 coral fragments analysed in this study produced bulk-cell DGGE band patterns consistent with the presence of Symbiodinium C100, either in apparent isolation or in coexistence with Symbiodinium C109. No other Symbiodinium sequences were detected, including the rare types $\mathrm{C} 103$ and $\mathrm{C} 118$ previously identified from $P$. damicornis at LHI (Wicks et al. 2010). The relative C100 and C109 band intensities generally corresponded with the relative proportions of each ITS2 type, as determined by bulk-cell qPCR (see Appendix B). Conversion of bulk-cell ITS2 ratios $\left(\mathrm{C}_{\mathrm{C} 100}: \mathrm{C}_{\mathrm{TOTAL}}\right)$ to the proportional abundance of genetically heterogeneous symbionts $\left(S_{\mathrm{H}}: S_{\mathrm{T}}\right)$ revealed that genetically heterogeneous Symbiodinium cells constituted between $0.15 \%$ and $0.72 \%$ of the symbiont consortium (Figure 4.4). The variation in $S_{\mathrm{H}}: S_{\mathrm{T}}$ between fragments sampled from the same colony was negligible, despite experiencing a $4{ }^{\circ} \mathrm{C}$ difference in temperature for $4 \mathrm{~d}(7.7 \%$ residual variance component; Figure 4.4). 


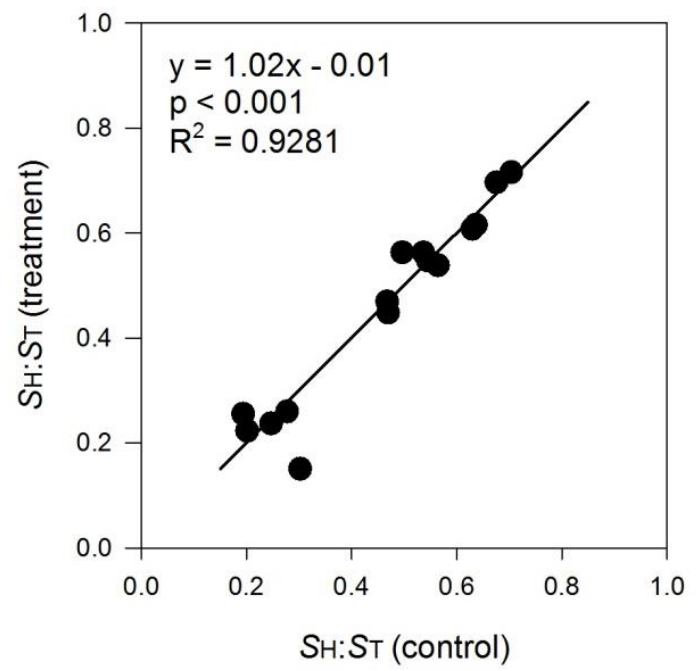

Figure 4.4 Within-colony variation in symbiont abundance ratios

Similar $S_{\mathrm{H}}: S_{\mathrm{T}}$ ratios between control $\left(25^{\circ} \mathrm{C}\right)$ and treatment $\left(29^{\circ} \mathrm{C}\right)$ fragments reflect a lack of intracolony variation in the proportional abundance of genetically heterogeneous symbionts following a $4 \mathrm{~d}$ exposure to different temperatures. A 7.7\% residual variance component (calculated as $1-$ $\mathrm{R}^{2}$ ) corresponds with the low between-branch variability observed in chapters 2 and 3, and suggests that the coral host does not selectively expel symbionts over short time-frames.

\subsubsection{Morphology and physiology}

Temperature treatment and $S_{\mathrm{H}}: S_{\mathrm{T}}$ jointly explained a significant proportion of the variation in the multi-parameter physiological response of $P$. damicornis (MANCOVA, Pillai's Trace pvalue $=0.029$ and 0.004 for covariate and treatment effects, respectively; Table 4.1). Subsequent univariate testing revealed a significant main effect of $S_{\mathrm{H}}: S_{\mathrm{T}}$ on the majority of the variables measured, either in isolation or in conjunction with temperature (Table 4.2). The total soluble protein biomass per unit surface area (used here as a proxy for host tissue thickness) was unaffected by temperature $(\mathrm{p}=0.746)$ and was not significantly correlated with $S_{\mathrm{H}}: S_{\mathrm{T}}$. However, a trend towards a higher tissue biomass in corals hosting more putative hybrids was observable ( $p=0.075$; Figure 4.5a; Table 4.2). Symbiont density was negatively correlated with $S_{\mathrm{H}}: S_{\mathrm{T}}$ when normalised to both host protein biomass and coral surface area. A $1 \%$ increase in $S_{\mathrm{H}}: S_{\mathrm{T}}$ was associated with approximately 13,500 fewer symbiont cells per mg host protein, regardless of temperature $(\mathrm{p}=0.002$; Figure $4.5 \mathrm{~b})$. Similarly, a $1 \%$ increase in $S_{\mathrm{H}}: S_{\mathrm{T}}$ corresponded to $\sim 3,000$ fewer symbiont cells per $\mathrm{cm}^{2}(\mathrm{p}=0.023$; Figure $4.5 \mathrm{c})$. However, in contrast to the host protein-normalized symbiont density, the density of Symbiodinium cells per unit surface area was also dependent on temperature, with corals acclimated at $25^{\circ} \mathrm{C}$ maintaining around 120,000 more symbiont cells per $\mathrm{cm}^{2}$ than those kept at $29{ }^{\circ} \mathrm{C}(\mathrm{p}=0.012$; Figure $4.5 \mathrm{c})$. A reduced symbiont density in corals hosting proportionally more putative Symbiodinium hybrids was consistent with an observed difference in symbiont cell size ( $p=0.022$; Figure $4.5 \mathrm{~d})$; extrapolation from the MANCOVA fitted regression line indicated that the average diameter of genetically heterogeneous 
symbionts was $11.53 \mu \mathrm{m}$, compared to $10.15 \mu \mathrm{m}$ for the homogeneous cells (corresponding cell volume $=803 \mu \mathrm{m} 3$ and $548 \mu \mathrm{m}^{3}$ respectively).
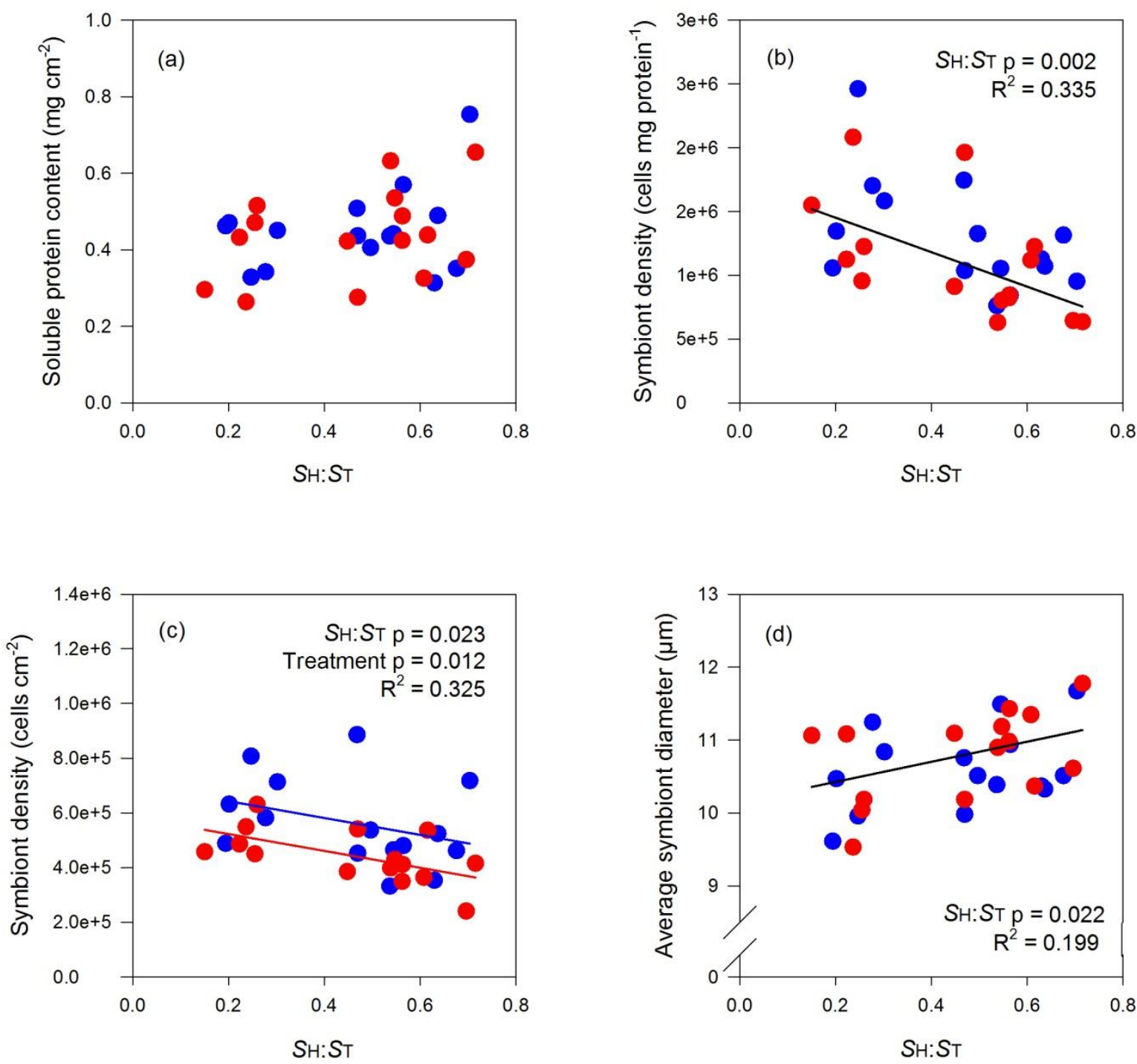

Figure 4.5 Host protein biomass, symbiont density and cell-size

Differences in morphology and physiology were evident between genetically homogeneous and heterogeneous symbionts, and between high- and low- $S_{\mathrm{H}}: S_{\mathrm{T}}$ colonies, with (a) similar host protein biomass among colonies and temperature treatments (although a positive trend was indicated by a near-significant p-value of 0.075); (b) reduced protein-normalised symbiont densities in high- $S_{\mathrm{H}}: S_{\mathrm{T}}$ colonies; (c) reduced surface area-normalised symbiont densities in high- $S_{\mathrm{H}}: S_{\mathrm{T}}$ colonies, that were further reduced in all colonies at elevated temperature; and (d) larger average symbiont cell diameters in high- $S_{\mathrm{H}}: S_{\mathrm{T}}$ colonies (extrapolated estimates $=11.53 \mu \mathrm{m}$ and $10.15 \mu \mathrm{m}$ for genetically heterogeneous and homogeneous symbiont cells, respectively). Blue and red circles represent coral fragments following exposure to $25^{\circ} \mathrm{C}$ and $29^{\circ} \mathrm{C}$, respectively. Coloured lines show the ANCOVA model when both $S_{\mathrm{H}}: S_{\mathrm{T}}$ and the temperature produced significant effects. A single sloping black line is fitted in cases where temperature effect was non-significant (i.e. reduced to a linear regression), and a single horizontal black line indicates an absence of both $S_{\mathrm{H}}: S_{\mathrm{T}}$ and temperature effects. 
The total chlorophyll $a$ content per Symbiodinium cell was similar across all coral fragments, regardless of $S_{\mathrm{H}}: S_{\mathrm{T}}$ and temperature $\left(\mathrm{p}=0.221\right.$ and 0.497 for $S_{\mathrm{H}}: S_{\mathrm{T}}$ and treatment effects, respectively; mean $=3.36 \mathrm{pg}$ cell $^{-1}$; Figure $4.6 \mathrm{a}$ ). By contrast, the symbionts of colonies hosting comparatively more genetically heterogeneous Symbiodinium cells contained more chlorophyll $c_{2}$ per cell than those of low- $S_{\mathrm{H}}: S_{\mathrm{T}}$ colonies, with a $1 \%$ increase in $S_{\mathrm{H}}: S_{\mathrm{T}}$ conferring an increase of $0.614 \mathrm{pg} \mathrm{cell}^{-1}$ ( $\mathrm{p}=0.006$; Figure 4.6b). As such, genetically heterogeneous symbionts featured a correspondingly higher chlorophyll $c_{2}: a$ ratio than homogeneous cells (extrapolated estimates $=0.50$ for putative hybrids and 0.25 for nonhybrids; $\mathrm{p}=0.002$; Figure $4.6 \mathrm{c}$ ). The chlorophyll $c_{2}$ composition also showed an increase at elevated temperature, with treated symbiont cells containing an additional $0.18 \mathrm{pg}$ chlorophyll $c_{2}$ at the end of the 4-d acclimation period ( $\mathrm{p}=0.019$; Figure 4.6b). However, this temperature effect was not of sufficient magnitude to significantly increase the $c_{2}: a$ ratio $(\mathrm{p}=0.248 ;$ Figure 4.6c). 

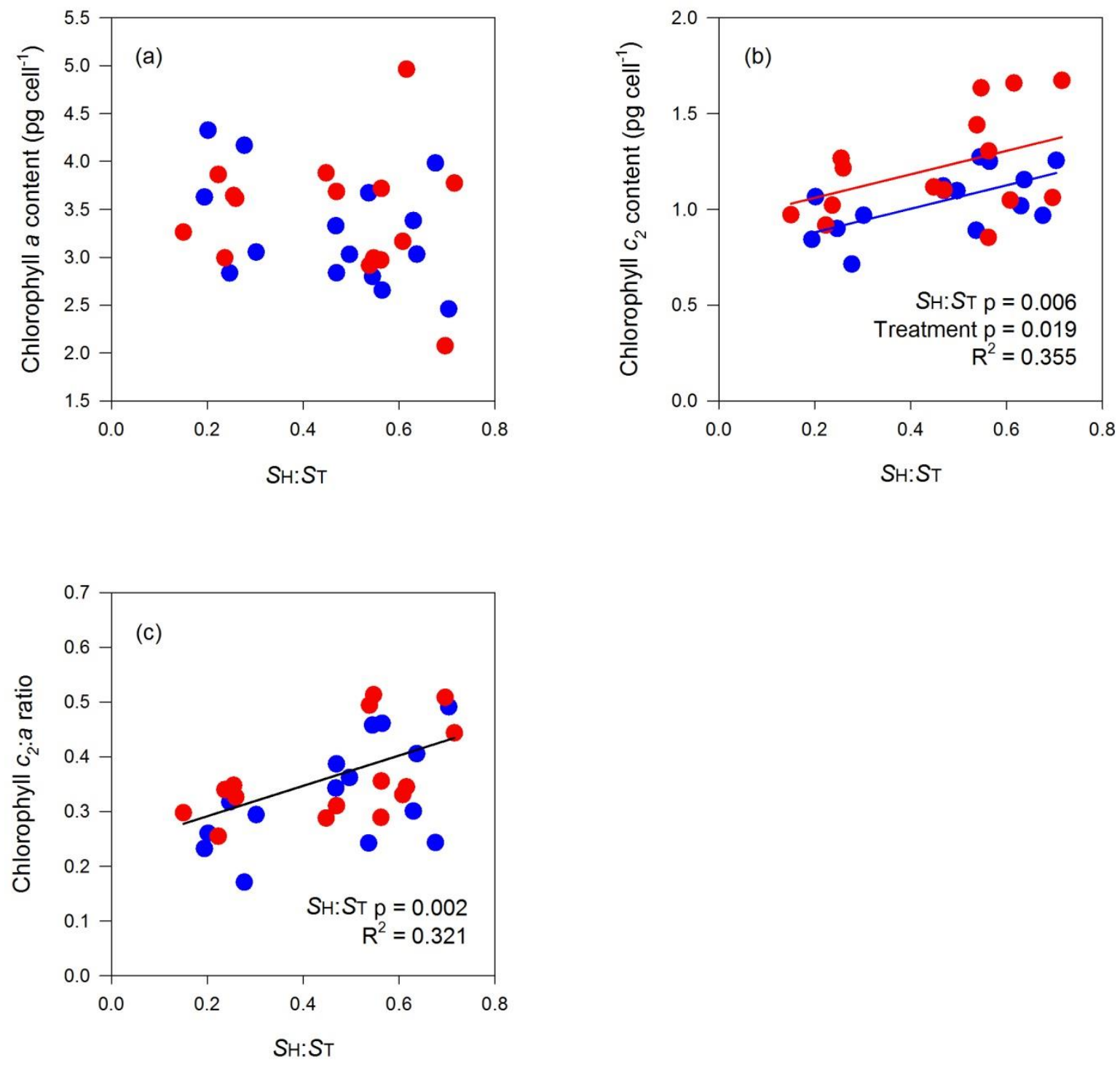

Figure 4.6 Chlorophyll $a$ and $c_{2}$ content

The chlorophyll content per-symbiont cell varied among colonies and treatments, with (a) no effect of either $S_{\mathrm{H}}: S_{\mathrm{T}}$ or temperature on the content of chlorophyll $a$ per cell; (b) a positive correlation between $S_{\mathrm{H}}: S_{\mathrm{T}}$ and the chlorophyll $c_{2}$ content per cell (with a further positive effect of elevated temperature); and (c) increased chlorophyll $c_{2}: a$ ratios in colonies dominated by genetically heterogeneous symbionts. Blue and red circles represent coral fragments following exposure to $25^{\circ} \mathrm{C}$ and $29^{\circ} \mathrm{C}$, respectively. Coloured lines show the ANCOVA model when both $S_{\mathrm{H}}: S_{\mathrm{T}}$ and the temperature produced significant effects. A single sloping black line is fitted in cases where temperature effect was non-significant (i.e. reduced to a linear regression), and a single horizontal black line indicates an absence of both $S_{\mathrm{H}}: S_{\mathrm{T}}$ and temperature effects. 


\subsubsection{Photosynthetic efficiency}

All photosynthesis-irradiance parameters were influenced by the proportional abundance of genetically heterogeneous symbionts, either alone or in conjunction with temperature (Figure 4.7; Figure 4.8$)$. The maximum gross photosynthetic rate $\left(P_{G R O S S}\right)$ was not affected by the temperature treatment $(\mathrm{p}=0.972)$, but declined by $16 \mathrm{nmol} \mathrm{O}_{2} \mathrm{mg}^{-1}$ protein $\mathrm{h}^{-1}$ with each $1 \%$ increase in $S_{\mathrm{H}}: S_{\mathrm{T}}(\mathrm{p}=0.003$; Figure $4.7 \mathrm{a})$. The net oxygen uptake in the dark (respiration; $R$ ) similarly declined by $6 \mathrm{nmol} \mathrm{O} \mathrm{mg}^{-1}$ protein $\mathrm{h}^{-1}$ for each $1 \%$ increase in $S_{\mathrm{H}}: S_{\mathrm{T}}(\mathrm{p}=0.041)$. However, in contrast to $P_{G R O S S}, R$ was also influenced by temperature, with fragments exposed to $29{ }^{\circ} \mathrm{C}$ consuming an additional $0.245 \mu \mathrm{mol} \mathrm{O} \mathrm{mg}^{-1}$ protein $\mathrm{h}^{-1}$ relative to the controls $(\mathrm{p}=0.024 ;$ Figure $4.7 \mathrm{~b})$. As such, the maximum photosynthesis to respiration ratio $(P: R)$ was dependent on both temperature and the proportional abundance of genetically heterogeneous symbionts. A $1 \%$ increase in $S_{\mathrm{H}}: S_{\mathrm{T}}$ corresponded to a 5.14 x $10^{-3}$ unit linear reduction in $P: R$, and the temperature-treated fragments exhibited a 0.368 unit reduction in $P: R$ relative to the controls $\left(\mathrm{p}=0.003\right.$ and $\mathrm{p}<0.001$ for $S_{\mathrm{H}}: S_{\mathrm{T}}$ and temperature treatment, respectively; Figure $4.7 \mathrm{c}$ ).

Table 4.1 MANCOVA for multi-parameter physiological response

\begin{tabular}{llllll}
\hline Source & Pillai's Trace & Hypothesis df & Error df & F & P \\
\hline $\boldsymbol{S}_{\mathrm{H}}: \boldsymbol{S}_{\mathbf{T}}$ & 0.677 & 12 & 16 & 2.791 & 0.029 \\
Treatment & 0.793 & 12 & 16 & 5.109 & 0.002 \\
\hline$S_{\mathrm{H}}: \boldsymbol{S}_{\mathrm{T}}$ was
\end{tabular}

$S_{\mathrm{H}}: S_{\mathrm{T}}$ was entered as a continuous covariate, and the temperature treatment entered as a fixed factor (at two levels, control and elevated).

An increase in the proportional abundance of genetically heterogeneous symbionts corresponded with a decline in light harvesting efficiency $(\alpha ; \mathrm{p}=0.001)$. However, this parameter remained unaffected by the temperature treatment $(p=0.831$; Figure $4.7 d)$. The irradiance required to produce net oxygen evolution (compensation irradiance; $I_{C}$ ) was significantly higher in corals hosting a relatively higher abundance of genetically heterogeneous Symbiodinium cells $(\mathrm{p}=0.012)$, with an increase of $0.76 \mu \mathrm{mol}$ photons $\mathrm{m}^{-2} \mathrm{~s}^{-1}$ for each $1 \%$ increase in $S_{\mathrm{H}}: S_{\mathrm{T}}$. The average $I_{C}$ increased by a further $32 \mu \mathrm{mol}$ photons $\mathrm{m}^{-2} \mathrm{~s}^{-1}$ in fragments exposed to $29{ }^{\circ} \mathrm{C}$ relative to those kept at $25^{\circ} \mathrm{C}(\mathrm{p}=0.003$; Figure 4.7e). Finally, the $50 \%$-saturation light intensity $\left(I_{K}\right)$ declined by $0.402 \mu \mathrm{mol}$ photons $\mathrm{m}^{-2} \mathrm{~s}^{-1}$ for 
every $1 \%$ increase in $S_{\mathrm{H}}: S_{\mathrm{T}}(\mathrm{p}=0.038)$, but was not affected by temperature $(\mathrm{p}=0.786$; Figure 4.7f). 

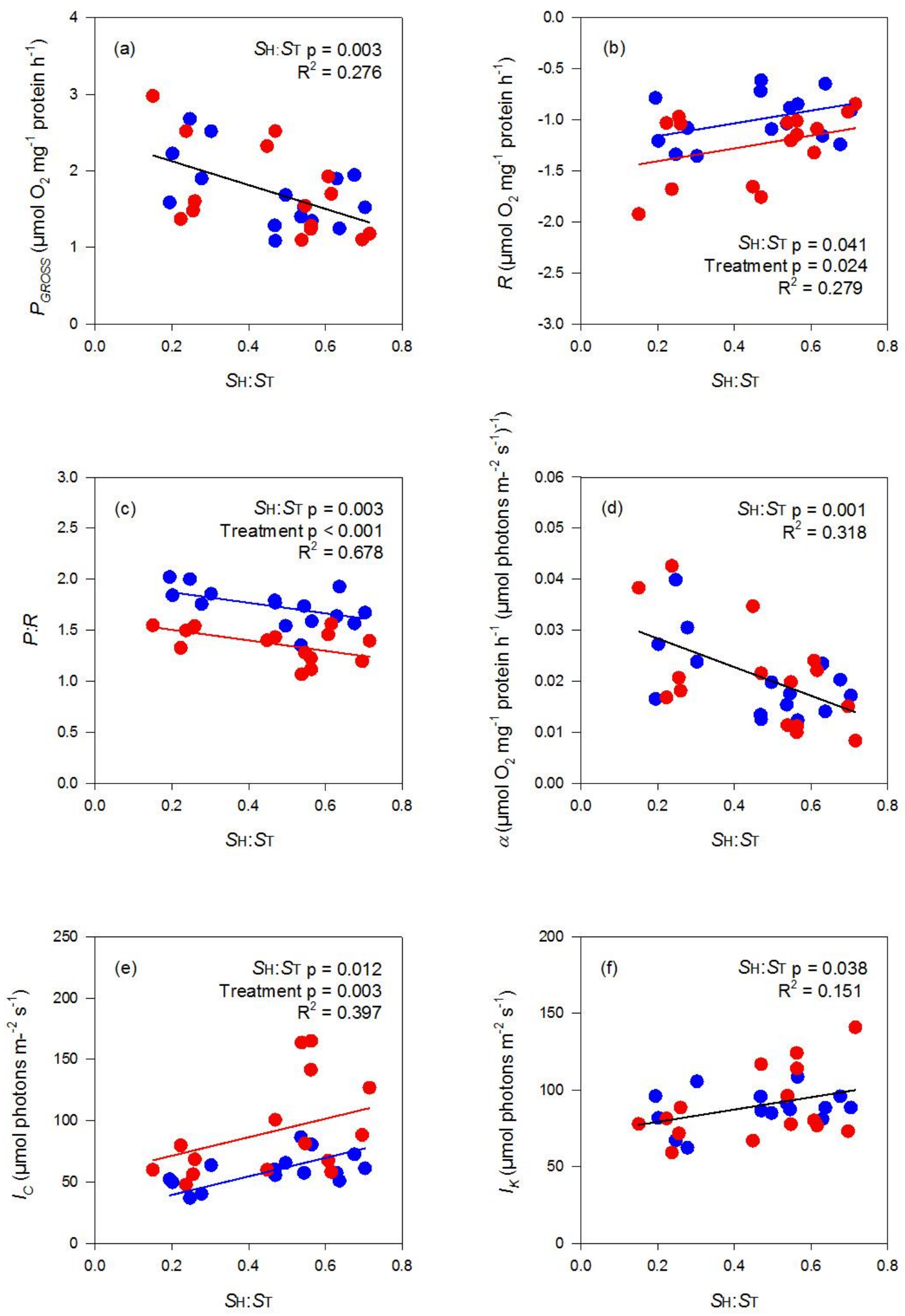


\section{Figure 4.7 (previous page) Respiratory and photosynthetic oxygen fluxes}

The proportional abundance of genetically heterogeneous symbionts had an influence on all measured photosynthesis-irradiance parameters, with (a) a negative linear correlation between $S_{\mathrm{H}}: S_{\mathrm{T}}$ and maximum gross photosynthesis; (b) an increase in respiratory oxygen consumption in low- $S_{\mathrm{H}}: S_{\mathrm{T}}$ colonies (further intensified in all colonies at elevated temperature); (c) disproportionately high photosynthetic rates relative to respiration in low- $S_{\mathrm{H}}: S_{\mathrm{T}}$ colonies, conferring an increase in $P: R$; (d) reduced light harvesting efficiency in genetically heterogeneous Symbiodinium cells, compared with homogeneous C100 symbionts; (e) an associated increase in compensation irradiance in high- $S_{\mathrm{H}}: S_{\mathrm{T}}$ colonies, particularly at the elevated temperature (corresponding with an increase in respiration); and (f) an increased $50 \%$-saturation irradiance in high- $S_{\mathrm{H}}: S_{\mathrm{T}}$ colonies. Blue and red circles represent coral fragments following exposure to $25^{\circ} \mathrm{C}$ and $29^{\circ} \mathrm{C}$, respectively. Coloured lines show the ANCOVA model when both $S_{\mathrm{H}}: S_{\mathrm{T}}$ and the temperature produced significant effects. A single sloping black line is fitted in cases where temperature effect was non-significant (i.e. reduced to a linear regression), and a single horizontal black line indicates an absence of both $S_{\mathrm{H}}: S_{\mathrm{T}}$ and temperature effects.
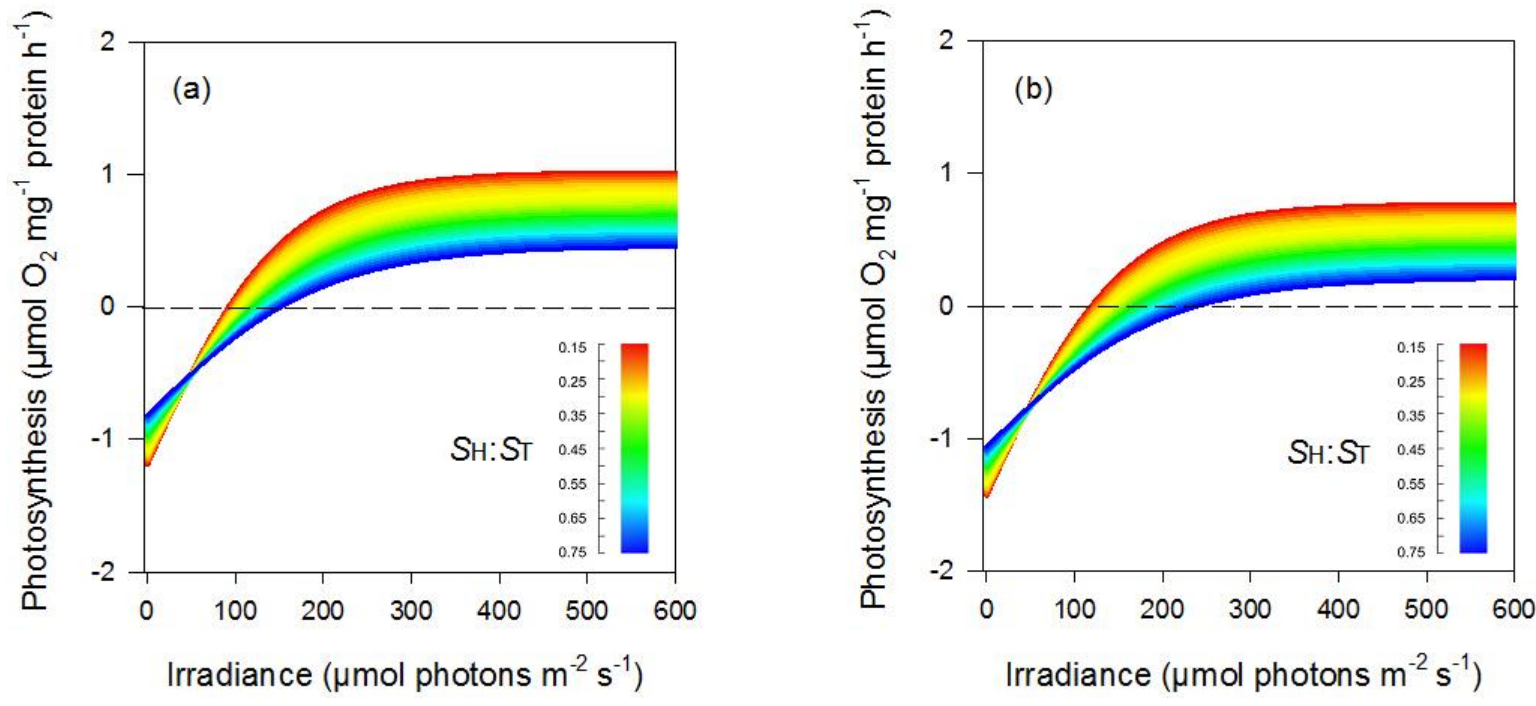

\section{Figure 4.8 Photosynthesis-irradiance model for $P$. damicornis}

The photosynthetic performance of $P$. damicornis is shown at ambient temperature (a) and under thermal stress (b), with the proportional abundance of genetically heterogeneous symbionts depicted as a colour gradient. Ambient and stressful thermal conditions are represented by the average midsummer temperature at North Bay $\left(25^{\circ} \mathrm{C}\right)$, and that slightly exceeding the thermal maximum experienced in the LHI lagoon $\left(29^{\circ} \mathrm{C}\right)$. An increase in $S_{\mathrm{H}}: S_{\mathrm{T}}$ was associated with a reduction in (i) maximum net photosynthetic oxygen evolution $\left(P_{G R O S S}\right)$; (ii) the irradiance required to reach $50 \%$ photosynthetic saturation $\left(I_{K}\right)$; (iii) the light harvesting efficiency $(\alpha)$; and (iv) the ratio of photosynthesis to respiration $(P: R)$. A corresponding increase was detected in the irradiance required to meet respiratory oxygen requirements $\left(I_{C}\right)$. The total oxygen uptake during respiration $(R)$ was lower in colonies hosting comparatively more genetically heterogeneous symbionts, corresponding with a reduced symbiont cell density. Colonies dominated by heterogeneous symbionts were only marginally capable of net photosynthesis at elevated temperature, even under saturating irradiance. 
Table 4.2 Univariate ANCOVA for individual physiological response variables

\begin{tabular}{|c|c|c|c|c|c|c|}
\hline Source & Dependent variable & df & SS & MS & $\mathbf{F}$ & $\mathbf{P}$ \\
\hline \multirow[t]{13}{*}{$S_{\mathrm{H}}: S_{\mathrm{T}}$} & Protein (surface area) & 1 & 0.041 & 0.041 & 3.418 & 0.075 \\
\hline & Density (protein) & 1 & $1.67901 \mathrm{E}+12$ & $1.67901 \mathrm{E}+12$ & 11.706 & 0.002 \\
\hline & Density (surface area) & 1 & 87313961439 & 87313961439 & 5.852 & 0.023 \\
\hline & Symbiont diameter & 1 & 1.745 & 1.745 & 5.873 & 0.022 \\
\hline & Chlorophyll $a$ (cell) & 1 & 0.572 & 0.572 & 1.572 & 0.221 \\
\hline & Chlorophyll $c_{2}$ (cell) & 1 & 0.346 & 0.346 & 8.827 & 0.006 \\
\hline & Chlorophyll $c_{2}: a$ & 1 & 0.07 & 0.07 & 12.096 & 0.002 \\
\hline & $P_{\text {GROSS }}$ (protein) & 1 & 2.22 & 2.22 & 10.301 & 0.003 \\
\hline & $R$ (protein) & 1 & 0.361 & 0.361 & 4.608 & 0.041 \\
\hline & $P: R$ & 1 & 0.243 & 0.243 & 11.063 & 0.003 \\
\hline & $\alpha$ (protein) & 1 & 0.001 & 0.001 & 12.53 & 0.001 \\
\hline & $I_{K}$ & 1 & 1491.185 & 1491.185 & 4.742 & 0.038 \\
\hline & $I_{C}$ & 1 & 5242.373 & 5242.373 & 7.305 & 0.012 \\
\hline \multirow[t]{13}{*}{ Treatment } & Protein (surface area) & 1 & 0.001 & 0.001 & 0.107 & 0.746 \\
\hline & Density (protein) & 1 & $2.86542 \mathrm{E}+11$ & $2.86542 \mathrm{E}+11$ & 1.998 & 0.169 \\
\hline & Density (surface area) & 1 & $1.08932 \mathrm{E}+11$ & $1.08932 \mathrm{E}+11$ & 7.301 & 0.012 \\
\hline & Symbiont diameter & 1 & 0.259 & 0.259 & 0.873 & 0.358 \\
\hline & Chlorophyll $a$ (cell) & 1 & 0.173 & 0.173 & 0.474 & 0.497 \\
\hline & Chlorophyll $c_{2}$ (cell) & 1 & 0.243 & 0.243 & 6.206 & 0.019 \\
\hline & Chlorophyll $c_{2}: a$ & 1 & 0.008 & 0.008 & 1.396 & 0.248 \\
\hline & $P_{\text {GROSS }}$ (protein) & 1 & 0 & 0 & 0.001 & 0.972 \\
\hline & $R$ (protein) & 1 & 0.45 & 0.45 & 5.743 & 0.024 \\
\hline & $P: R$ & 1 & 1.015 & 1.015 & 46.315 & $<0.001$ \\
\hline & $\alpha$ (protein) & 1 & $2.73 \mathrm{E}-06$ & $2.73 \mathrm{E}-06$ & 0.046 & 0.831 \\
\hline & $I_{K}$ & 1 & 23.742 & 23.742 & 0.076 & 0.786 \\
\hline & $I_{C}$ & 1 & 7657.407 & 7657.407 & 10.67 & 0.003 \\
\hline \multirow[t]{13}{*}{ Error } & Protein (surface area) & 27 & 0.326 & 0.012 & & \\
\hline & Density (protein) & 27 & $3.87274 \mathrm{E}+12$ & $1.43435 \mathrm{E}+11$ & & \\
\hline & Density (surface area) & 27 & $4.02825 \mathrm{E}+11$ & 14919426866 & & \\
\hline & Symbiont diameter & 27 & 8.022 & 0.297 & & \\
\hline & Chlorophyll $a$ (cell) & 27 & 9.833 & 0.364 & & \\
\hline & Chlorophyll $c_{2}$ (cell) & 27 & 1.058 & 0.039 & & \\
\hline & Chlorophyll $c_{2}: a$ & 27 & 0.157 & 0.006 & & \\
\hline & $P_{\text {GROSS }}$ (protein) & 27 & 5.82 & 0.216 & & \\
\hline & $R$ (protein) & 27 & 2.117 & 0.078 & & \\
\hline & $P: R$ & 27 & 0.592 & 0.022 & & \\
\hline & $\alpha$ (protein) & 27 & 0.002 & $5.86 \mathrm{E}-05$ & & \\
\hline & $I_{K}$ & 27 & 8490.012 & 314.445 & & \\
\hline & $I_{C}$ & 27 & 19376.082 & 717.633 & & \\
\hline
\end{tabular}

$S_{\mathrm{H}}: S_{\mathrm{T}}$ was entered as a continuous covariate, and the temperature treatment entered as a fixed factor (at two levels, control and elevated). Denominators for normalised variables are shown in parentheses. 


\subsection{Discussion}

The aim of this chapter was to investigate the form and function of genetically heterogeneous Symbiodinium cells (putative hybrids) in comparison to genetically homogeneous symbionts (putative progenitors), under ambient and stressful conditions. A suite of diagnostic variables were measured, with morphological and physiological differences observed at both the symbiont- and holobiont level. Consistent with the findings of the previous chapter, genetically heterogeneous symbionts performed poorly at ambient and elevated summer temperatures, with their host colonies suffering substantial reductions in photosynthetic efficiency. However, this did not prevent net photosynthetic production, even when the maximum temperature experienced in their natural habitat was exceeded.

\subsubsection{Physical attributes of putative Symbiodinium hybrids}

Genetically heterogeneous Symbiodinium cells were 50\% larger by volume (applying the sphere radius to volume conversion $V=4 / 3 \pi r^{3}$ ) than the homogeneous $\mathrm{C} 100$ symbionts. This disparity is likely to have an underlying genetic basis, since conspecific Symbiodinium cells generally show only minor variation in size (LaJeunesse 2001; LaJeunesse et al. 2012, 2014). Corals dominated by these large heterogeneous symbiont cells showed corresponding reductions in symbiont density. However, while two-dimensional space availability is known to be important factor in limiting algal densities in corals (Jones \& Yellowlees 1997), an estimated $10 \%$ difference in cell diameter did not entirely explain the two-fold difference in density observed between corals at opposite ends of the $S_{\mathrm{H}}: S_{\mathrm{T}}$ spectrum (i.e. $15 \%$ versus $75 \%$ proportional abundance of genetically heterogeneous symbionts). Symbionts are typically not space limited in corals during the summer months (Fitt et al. 2000), and P. damicornis colonies from other locations are known to accommodate a double layer Symbiodinium of cells at densities exceeding $10^{6} \mathrm{~cm}^{-2}$ (Gates \& Muscatine 1992; Muller-Parker et al. 1994). This suggests that the colonies dominated by genetically heterogeneous Symbiodinium cells were not 'symbiont saturated'. Rather, they appear to have previously experienced higher rates of symbiont loss, and/or suffered from lower symbiont division rates that may have been unable to maintain pace with terminal coral growth (e.g. Oliver 1984).

Rates of temperature-induced symbiont loss were similar among all colonies in this study, with a $4{ }^{\circ} \mathrm{C}$ increase in temperature resulting in the expulsion/degradation of an extra 
30,000 symbionts per $\mathrm{cm}^{2}$ per day. This meant that thermally-stressed colonies dominated by genetically heterogeneous Symbiodinium cells lost a greater percentage of their symbionts than those hosting predominantly homogeneous C100 cells (since the former initially hosted fewer symbionts). Under prolonged thermal stress, high- $S_{\mathrm{H}}: S_{\mathrm{T}}$ colonies may therefore exhaust their energy reserves sooner unless they can compensate by switching to a heterotrophic feeding mode (e.g. Grottoli et al. 2006). Interestingly, colonies dominated by genetically heterogeneous symbionts showed a high protein biomass, even in comparison to nitrogenenriched P. damicornis colonies (Muller-Parker et al. 1994). Similar increases in protein biomass were observed in Hawaiian Montipora capitata colonies that switched to heterotrophy after bleaching (Grottoli et al. 2006), suggesting a possible role of heterotrophic feeding in the survival of the corals analysed here. The influence of symbiont hybridization on the feeding behaviour and morphology of the coral host presents an interesting area for future research.

The concentration of the primary photosynthetic pigment chlorophyll $a$ was similar among genetically heterogeneous and homogeneous (C100) Symbiodinium cells. However, the former showed a substantially higher content of the accessory pigment chlorophyll $c_{2}$ and a corresponding two-fold increase in the $c_{2}: a$ ratio, suggesting inherent differences in the composition of light harvesting antennae (membrane-bound chlorophyll $a$-chlorophyll $c_{2^{-}}$ peridinin-protein complexes) between the two cell types. The stoichiometry of lightharvesting antennae varies widely among symbiont taxa, with species-specific differences explained by a combination of adaptation and acclimatization to different habitats (Chang et al. 1983; Iglesias-Prieto \& Trench 1994, 1997). For example, in the symbiont of the shadeadapted coral Montipora verrucosa (Symbiodinium kawagutii; type F1), $c_{2}$ : a was negatively correlated with light intensity (Chang et al. 1983; Iglesias-Prieto \& Trench 1994), while $c_{2}: a$ showed a positive correlation with light intensity in the clade A symbiont of the light-adapted giant clam Tridacna maxima (Chang et al. 1983). The differences in $c_{2}: a$ observed here may represent a heritable modification; however dynamic changes in light-harvesting antennae can also occur within individual cells, through remodelling, de novo synthesis, or differential degradation (Iglesias-Prieto \& Trench 1997). It is therefore unclear whether the observed differences in $c_{2}: a$ represents an adaptive (i.e. genetically encoded) modification, an acclimatory response to subtle differences in microhabitat, or a combination of both processes. The role(s) of accessory pigments in the ecological diversification of genetically 
homogeneous and heterogeneous Symbiodinium cells presents another area for future investigation.

\subsubsection{Relative photosynthetic performance of putative Symbiodinium hybrids}

Corals dominated by genetically heterogeneous Symbiodinium cells showed lower overall rates of gross photosynthesis, respiration, and $P: R$ than those predominantly hosting genetically pure C100 symbionts. This loss of photosynthetic performance can be partially attributed to the reduced symbiont densities in these colonies (see above). However, a corresponding reduction in light-harvesting efficiency can be entirely attributed to the photosynthetic function of the symbiont. Symbiont populations at low density generally show increased light harvesting efficiency, reduced compensation irradiance, and higher rates of net photosynthesis (Crossland \& Barnes 1977; Muller-Parker 1984). For example, freshly isolated symbionts from the coral Acropora acuminata showed high light-harvesting efficiency when maintained at a low density, while this efficiency declined at higher cell concentrations and in the intact symbiosis (as a result of shading; Crossland \& Barnes 1977). By contrast, high- $S_{\mathrm{H}}: S_{\mathrm{T}}$ colonies (hosting low symbiont densities), showed poor lightharvesting efficiency, and a correspondingly high irradiance was required to meet the comparatively lower overall oxygen demands of the symbiosis. While the underlying cellular mechanisms of this disparity remain unknown, the impaired photosynthetic performance of these genetically heterogeneous cells is consistent with 'hybrid breakdown', a reduction in performance and fitness due to the segregation of co-adapted gene complexes or the creation of maladapted gene combinations (Demuth \& Wade 2005). Haploids such as Symbiodinium are especially prone to hybrid breakdown, since the deleterious effects of recombination cannot be compensated for by heterosis (Barton 2001). Furthermore, while 'transgressive' hybrids can outperform parental genotypes in extreme habitats (Rieseberg et al. 2003), hybrids are generally expected to function poorly in the parental habitat. This is consistent with an overall reduction in performance shown by the genetically heterogeneous symbionts under conditions simulating those within the LHI lagoon (the average and absolute summer thermal maximum) where homogeneous $\mathrm{C} 100$ cells tend to be more dominant. While it remains a possibility that the observed physiological disparities arose from fine-scale genetic structure among sympatric asexual Symbiodinium populations, the poor photosynthetic performance of genetically heterogeneous Symbiodinium cells reinforces their candidacy as 
putative hybrids, and suggests that reduced symbiont fitness (and hence host fitness) may accompany the occurrence of hybridization between divergent Symbiodinium taxa.

\subsubsection{Implications of reduced performance in putative Symbiodinium hybrids}

Corals dominated by putative Symbiodinium hybrids suffered relatively poor overall photosynthetic function under both ambient and extreme summer temperatures. Extrapolation from the photosynthesis irradiance model suggests that a further marginal temperature increase beyond $29^{\circ} \mathrm{C}$ would render this symbiosis incapable of net oxygen production, even under saturating irradiance. This would likely result in substantial loss of fitness for both the host and the symbiont, raising several questions about the potential functional and evolutionary role(s) of hybridization in the Symbiodinium genus. First, do hybrids outperform non-hybrids under extreme and/or intermediate conditions (i.e. those between the parental optima)? The putative hybrids studied here may be more suitably adapted to cooler conditions, given that thermal stress appears to drive their distribution patterns (see chapter 3). A physiological assessment over a broader range of temperature and light conditions may reveal 'redeeming' features that offset their relatively poor performance under the conditions simulated in this study. Second, how does the observed physiological performance distribute among the different genotypes and classes (i.e. $\mathrm{F}_{1}$ hybrid, $\mathrm{F}_{2}$ backcross, etc)? If hybridization has occurred among the symbionts of $P$. damicornis, the cluster of genetically heterogeneous cells may solely represent $\mathrm{F}_{1}$ hybrids, or it may also include second- and later-generation backcross genotypes. Backcross genotypes are expected to suffer poor performance relative to the $F_{1}$ generation, and hence suffer further reductions in fitness (Barton 2001). Furthermore, the functional performance of the putative C109 progenitor remains unknown, since this type has only been detected forming a cryptic population in $P$. damicornis $(<7 \%$ proportional representation; see chapter 2). Establishing clonal cultures of the various genotypes would be necessary to address the fine-scale variation in both performance and fitness, and the underlying physiological mechanisms. Finally, does the observed disparity in physiological performance translate to differences in coral growth, survival and reproduction? Symbiont shuffling is not evident in this symbiosis (see chapter 3), suggesting that an individual coral's symbiont compliment is either inherited or determined early in its ontogeny. Natural selection acting on the coral host may therefore play an important role in 
determining the distribution of genetically homogeneous and heterogeneous Symbiodinium cells. This consideration will be addressed in chapter 5 .

\subsubsection{Conclusion}

Genetically heterogeneous and homogeneous Symbiodinium cells were morphologically and physiologically distinct, both in terms of cell size and their constituent photosynthetic pigments. The former also showed poor light-harvesting efficiency, despite being subject to less shading (resulting from low symbiont densities). These physiological differences translated into functional disparities in the coral holobiont, with colonies dominated by genetically heterogeneous Symbiodinium cells showing disproportionately low symbiont densities and poor photo-physiological performance under both ambient and elevated summer temperatures. These results add to the accumulating evidence in favour of hybridization between divergent Symbiodinium taxa, and provide a conditional indication of the performance and fitness of the resulting progeny. This is an important step towards understanding the potential for hybridization to contribute to adaptation in a changing climate. 


\section{Chapter 5: Symbiont hybridization and coral fitness}

\subsection{Introduction}

In obligate mutualisms, the performance of the symbiont is a key determinant of host fitness. Corals are no exception, since the photosynthetic activity of the algal symbiont ultimately supports the energetic requirements of the host through the translocation of energy-rich compounds (Muscatine \& Porter 1977; Muscatine 1990; Davy et al. 2012). Yet hosting symbionts inevitably incurs a basal metabolic cost (Grube et al. 2010), and as such, poorperforming symbionts are expected to occupy a less-beneficial position along the parasitismmutualism continuum (Cantin et al. 2009). For example, under normal conditions, a twofold increase in photosynthate translocation within juvenile Acropora millepora colonies hosting Symbiodinium $\mathrm{C} 1$ (= S. goreauii) resulted in substantially higher growth rates than those harbouring clade D (Little et al. 2004; Cantin et al. 2009; Jones \& Berkelmans 2010). Symbiont performance is also an important determinant of host fitness under stressful conditions. There is a firm link between the thermal optimum of the symbiont and the coral host's resistance or susceptibility to bleaching-related mortality (Rowan 2004; Berkelmans \& van Oppen 2006; Sampayo et al. 2008). However, emerging evidence suggests that the physiological and behavioural attributes of the host can be at least as important as those of the symbiont in determining thermal stress tolerance (e.g. Fitt et al. 2009; Paxton et al. 2013; Hawkins et al. 2014). For example, in Pocillopora damicornis and Acropora millepora colonies subjected to elevated temperatures, nitric oxide accumulation and host-cell apoptosis preceded symbiont dysfunction by several days (Hawkins et al. 2014). Expelled symbionts can be healthy and photosynthetically active upon expulsion, further suggesting that photosynthetic breakdown does not always precede or cause the bleaching response (Bhagooli \& Hidaka 2004; Hill \& Ralph 2007). Also, some coral species such as Montipora capitata can survive the loss of symbionts by switching to a predominantly heterotrophic feeding mode (Grottoli et al. 2006), providing another situation where symbiont performance may be uncoupled from the fitness of host. Indeed, high-performing symbionts can even be detrimental to host fitness. Excessive symbiont reproduction in situ can lead to burgeoning densities, and an increased bleaching risk via a density-dependent signalling cascade (Cunning \& Baker 2013). In extreme cases, a functional mutualistic symbiont may even tend 
towards parasitism through preferential photosynthate retention and pathogenic proliferation within the host (Sachs \& Wilcox 2006; Stat et al. 2008b).

The genetically heterogeneous symbionts of $P$. damicornis (putative hybrids) showed relatively poor photosynthetic performance compared to their putative progenitor (Symbiodinium C100) in a controlled environment (see chapter 4). In particular, a comparative reduction in light-harvesting efficiency meant that higher levels of photosynthetically active radiation (PAR) were required to meet the overall oxygen demands (and hence energy demands) of the symbiosis. This suggests that symbiont hybridization may associate with negative fitness consequences for the host, by introducing poor-performing hybrids at the expense of highly mutualistic 'pure' genotypes. Alternatively, symbiont hybridization could be of benefit to the host, even at the cost of functional performance, if hybrids show reduced antagonistic/parasitic tendencies (Schardl \& Craven 2003). For example, asexual hybrid fungal symbionts of the cool-season grass family Poaceae are selected for by their hosts, since the sexually reproductive progenitor Epichloë sp. can 'hijack' the plant's reproductive structures in order to facilitate spore dispersal (choke disease; Schardl \& Clay 1997). Biological fitness is broadly defined as the ability of an individual to survive and contribute genes to the next generation (Maynard Smith 1998). Therefore, while short-term assessments of symbiont performance may give an indication of host fitness, establishing this link requires long-term assessments of host survival, growth and reproduction. This objective can only realistically be achieved in a natural setting (Arnold 1983).

This chapter aimed to establish the relationship between symbiont performance and host fitness on the reef, where many biotic and abiotic factors interact to determine coral survival, growth and fecundity (Correa \& Baker 2011). The specific objectives of this study were to establish whether observed disparities in functional performance between genetically homogeneous and heterogeneous Symbiodinium cells translate to differences in the growth and survival of their coral hosts. This was assessed through the reciprocal transplantation of coral colonies between two reef sites featuring different thermal characteristics, and corresponding differences in the proportional abundance of putative Symbiodinium hybrids. The hypothesis tested in this study was that colonies hosting higher proportions of genetically heterogeneous Symbiodinium cells show lower growth rates and an increased risk of mortality, compared to those predominantly hosting homogeneous symbionts. 


\subsection{Methods}

\subsubsection{Study location and species}

This study was carried out at two reef sites at Lord Howe Island (Australia), North Bay ($31.521,159.047)$ and the reef margin site known as The Arch $(-31.539,159.055$; Figure 5.1) The former is a sheltered inner-lagoon site that is subject to pronounced thermal maxima (see chapter 3). Here, P. damicornis colonies can grow to several metres in diameter and form large monoculture stands (Miller \& Ayre 2004). By contrast, The Arch is an exposed reefmargin location characterised by heavy wave action, where $P$. damicornis forms a small dense clump-like morphology (Veron 2000; Miller \& Ayre 2004). The Arch features a lower maximum temperature than North Bay, and P. damicornis colonies host a correspondingly higher proportional abundance of genetically heterogeneous Symbiodinium cells (putative hybrids; see chapter 3). The genomes of these symbionts feature both C100 and C109diagnostic ITS2 sequences (see chapter 2), and they show reduced light-harvesting efficiency compared to their putative progenitor (Symbiodinium C100) at both average- and maximumsummer temperatures $\left(25^{\circ} \mathrm{C}\right.$ and $29{ }^{\circ} \mathrm{C}$, respectively; see chapter 4$)$. Genetically heterogeneous Symbiodinium cells are also approximately $50 \%$ larger by volume, feature two-fold higher chlorophyll $c_{2}$ : $a$ ratios, and exist in lower densities in the host tissue compared to homogeneous Symbiodinium C100 cells. As such, $P$. damicornis colonies dominated by putative hybrids show lower overall photosynthesis-to-respiration ratios $(P: R$; see chapter 4). Symbiodinium cells with homogeneous C109 arrays are also found in $P$. damicornis colonies at LHI; however these symbionts only form a cryptic population $(<7 \%$ proportional abundance; see chapter 2). Therefore, this study invokes the assumption that Symbiodinium $\mathrm{C} 109$ forms a minor contribution to the overall photosynthetic activity of the symbiosis. 
(a)

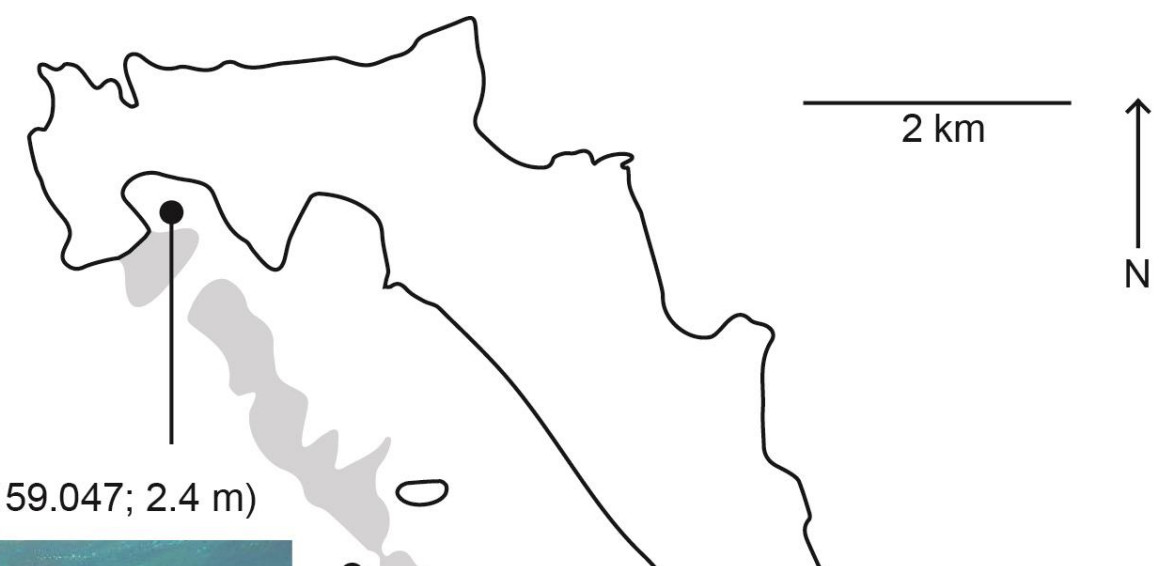

The Arch (-31.539, 159.055; $11.6 \mathrm{~m})$
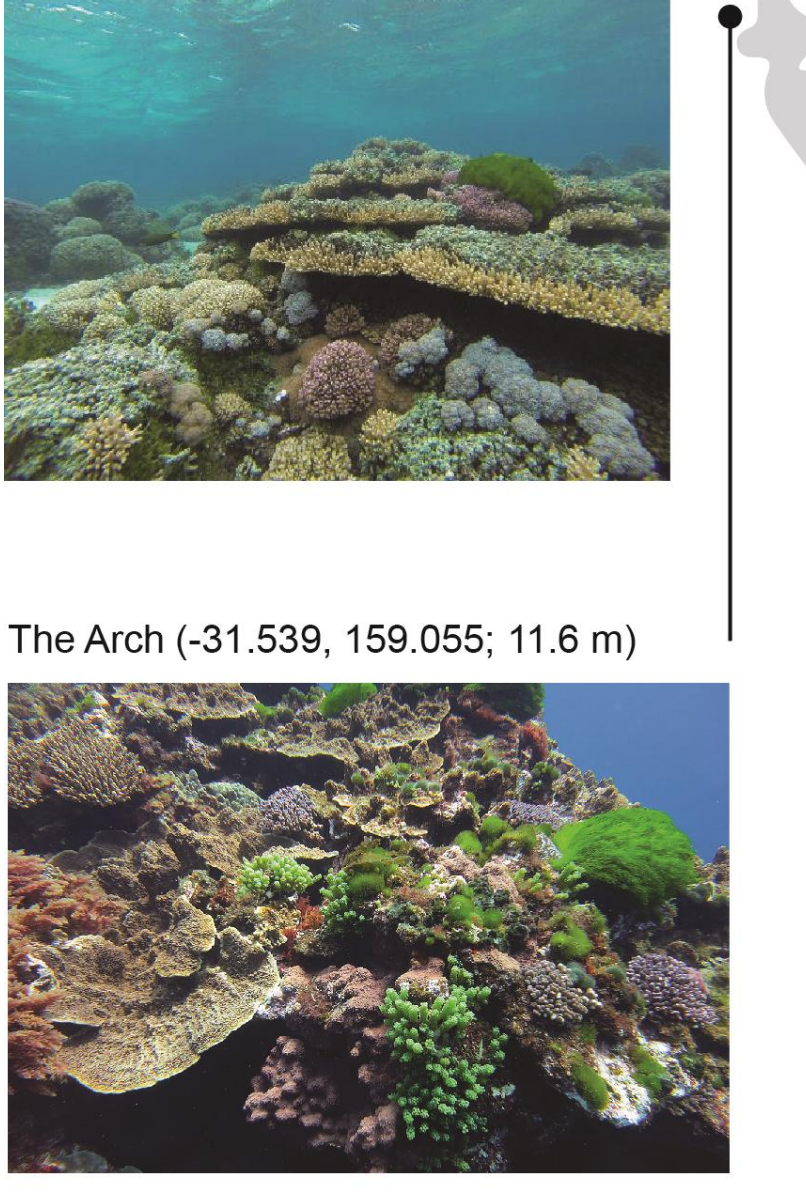

North Bay (-31.521, 159.047; 2.4 m)

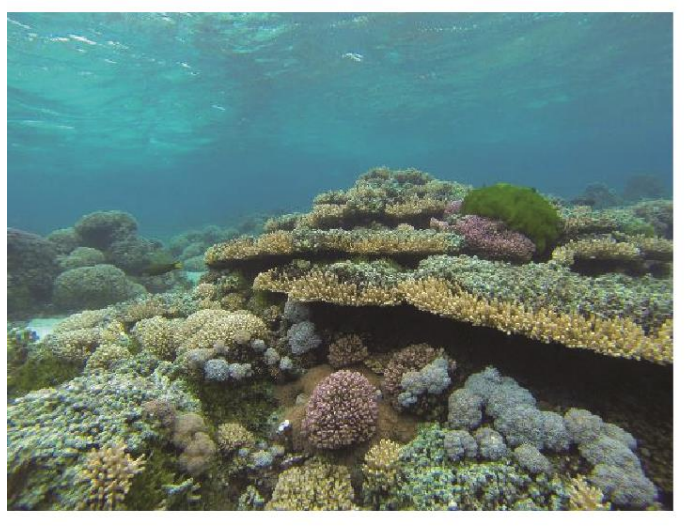




\section{Figure 5.1 (previous page) Location and method of coral transplantation}

Reciprocal transplantation of $P$. damicornis colonies was carried out between North Bay, a sheltered inner-lagoon site, and The Arch, an exposed reef-margin location (a). From each colony, three branch tips were transferred between sites and three were transplanted at the original site from where the parent colony was sampled. The transplantation method is shown in (b); coral fragments were attached to iron anchor weights using two zip ties, a transverse tie around the base of the fragment (red) and a longitudinal tie attached to the weight (green).

\subsubsection{Coral collection, transplantation and retrieval}

The initial collection of coral samples took place during September 2011, shortly after the winter thermal minimum. A single branch was collected from each of ten $P$. damicornis colonies at each site, and each branch was further divided into seven finger-sized terminal fragments (each approximately $7 \mathrm{~g}$ dry weight). One fragment was immediately frozen at -20 ${ }^{\circ} \mathrm{C}$ for DNA analysis, while the remaining six were weighed using the buoyant weight technique (Jokiel et al. 1978). These fragments were immediately transplanted onto the reef, three to the original site from where they were collected (transplanted controls) and three to the alternative site (i.e. from North Bay to The Arch or vice versa). Corals were attached to large cast-iron anchor weights using two plastic zip-ties, a transverse tie around the base of the fragment, and a larger longitudinal tie attached to the weight (Figure 5.1). The transplant sites were re-visited in March 2012 (shortly after the summer thermal maximum), and the identities of the fragments that had perished recorded. Two live fragments from each colony were retrieved (one from the original site and one from the new site), weighed (using the buoyant weight technique), and frozen at $-20{ }^{\circ} \mathrm{C}$ prior to DNA analysis. All remaining coral fragments were retrieved, weighed and frozen in September 2012.

\subsubsection{Skeletal mass estimation}

For each surviving coral fragment, the buoyant mass in water $\left(M_{W}\right)$ was converted to dry mass in air $\left(M_{A}\right)$ using the formula of (Jokiel et al. 1978): 


$$
M_{A}=\frac{M_{W}}{1-\left(\frac{D_{W}}{D_{M}}\right)}
$$

where $D_{W}$ is the density of seawater at a given temperature and salinity (calculated using oceanographic reference tables; UNESCO 1981), and $D_{M}$ is the species-specific aragonite density (2.703 $\mathrm{g} \mathrm{cm}^{-3}$ for P. damicornis; Spinaze et al. 1996). Larger coral fragments are expected to secrete comparatively more calcium (Ferrier-Pagès et al. 2000), therefore daily growth rates were converted to compounding growth rates (in $\left.\% \mathrm{~d}^{-1} ; G\right)$ using the formula:

$$
G=\left(\frac{M_{A}(\text { final })}{M_{A}(\text { initial })}\right)^{\frac{1}{t}}-1
$$

where $t$ is the time since transplantation (in d) and $M_{A}($ initial $)$ and $M_{A}($ final) are the initial and final estimated dry masses, respectively.

\subsubsection{DNA extraction}

DNA analysis of Symbiodinium ITS2 sequences in bulk-cell samples was similar to that described in chapters 3 and 4. For DNA analysis, a small branch tip was removed from each frozen coral fragment (surface area approximately $5-10 \mathrm{~cm}^{2}$ ). Coral tissue was removed from the underlying skeleton with a stream of $50 \mathrm{mM}$ phosphate buffer $(1 \mathrm{mM}$ EDTA; $\mathrm{pH}=7.8)$, delivered at high velocity through a compressed-air-generated spray nozzle. The homogenate was centrifuged at $16,100 \mathrm{x} \mathrm{g}$ for $5 \mathrm{~min}$ to pellet the Symbiodinium cells, and the supernatant was discarded. DMSO preservation buffer (20\% DMSO, 250 mM EDTA, NaCl saturated, pH 8.0; Seutin et al. 1991) was added to a volume of $200 \mu 1$, and the pellet was re-suspended by milling without beads for $3 \mathrm{~min}$ at $50 \mathrm{~Hz}$. Following a $7 \mathrm{~d}$ incubation at $-20{ }^{\circ} \mathrm{C}$, the settled Symbiodinium cells were re-suspended using a vortex, and $10 \mu \mathrm{l}$ of suspension were taken for DNA extraction. Acid-washed glass beads were added (50 mg; 710-1180 $\mu \mathrm{m}$; SigmaAldrich) and the sample was milled at $50 \mathrm{~Hz}$ for 3 min (Qiagen TissueLyser LT; Qiagen) to disrupt the cell-walls and enable the release of nucleic acids. Following a 10 min incubation period at $4{ }^{\circ} \mathrm{C}, 90 \mu \mathrm{l}$ of de-ionised water were added. The sample was then vortexed and centrifuged for $10 \mathrm{~min}$ at $16,100 \mathrm{x} \mathrm{g}$ to pellet the cellular debris. The DNA-enriched supernatant $(50 \mu \mathrm{l})$ was transferred to a new micro-centrifuge tube with an equal volume of 2-propanol to precipitate the nucleic acids, and centrifuged for $10 \mathrm{~min}$ at $16,100 \mathrm{x} \mathrm{g}$ to collect 
the DNA pellet. The supernatant was discarded and $200 \mu \mathrm{l}$ wash buffer (70\% v/v ethanol) were added. The sample was vortexed and returned to the centrifuge for a further $10 \mathrm{~min}$ at $16,100 \mathrm{x}$. The supernatant was carefully removed with a pipette and the DNA pellet was dried under a laminar flow hood for $30 \mathrm{~min}$. Elution buffer (10 mM Tris-HCl; $0.1 \mathrm{mM}$ EDTA; $\mathrm{pH}=8.0$ ) was added to a final volume of $50 \mu 1$. Following a $10 \mathrm{~min}$ re-hydration period, the DNA was re-suspended by milling without beads for 1 min at $30 \mathrm{~Hz}$.

\subsubsection{PCR and denaturing gradient gel electrophoresis}

Denaturing gradient gel electrophoresis (DGGE) was carried out on all samples to establish the presence or absence of Symbiodinium types other than C100 and C109. PCR amplification for DGGE was carried out with the primers ITSintfor2 and ITS2CLAMP (LaJeunesse 2002). Thermal cycling involved an initial denaturation step of 3 min at $95{ }^{\circ} \mathrm{C}$ followed by 40 cycles of 15 seconds at $95{ }^{\circ} \mathrm{C}, 15$ seconds at $56{ }^{\circ} \mathrm{C}$ and 10 seconds at $72{ }^{\circ} \mathrm{C}$ (carried out on an Applied Biosystems Veriti thermo-cycler; Life Technologies). Each reaction contained $4 \mu \mathrm{l}$ of DNA template solution, 1x MyTaq PCR reaction mix (Bioline), 10 pmol each primer, $10 \mu \mathrm{g}$ bovine serum albumin (BSA; Sigma) and deionised sterile water to a total volume of $20 \mu 1$. A template-free control was included with each run. To ensure the final product was within the intended size range (300-350 nucleotides), and that amplification did not occur in the template-free control reaction, $5 \mu 1$ of the final PCR product were electrophoresed on a $1.5 \%$ agarose gel containing 1x SYBR safe nucleic acid stain (Life Technologies) alongside a DNA standard (Hyperladder II; Bioline). Agarose gels were viewed and imaged on a blue light trans-illuminator (Safe Imager; Invitrogen).The remaining PCR product was loaded on a $200 \times 200 \times 1 \mathrm{~mm}, 8 \%$ denaturing polyacrylamide gel (25-50\% urea/formamide denaturant gradient), and run in $1 \mathrm{x} \mathrm{TAE}$ at $150 \mathrm{~V}$ for $7 \mathrm{~h}$ at $60{ }^{\circ} \mathrm{C}$ (DCode system; BioRad) alongside known ITS2 sequences of Symbiodinium C100 and C109 (PCRamplified from plasmid DNA). Following electrophoresis, denaturing gels were stained with ethidium bromide and viewed on a UV trans-illuminator (FirstLight UVP), and the presence or absence of each ITS2 sequence type was scored. 


\subsubsection{Quantitative PCR}

The bulk-cell qPCR assay developed in chapter 3 was used to establish the relative proportion of putative hybrids within the symbiont consortium of each coral fragment $\left(S_{\mathrm{H}}: S_{\mathrm{T}}\right)$. Each qPCR reaction contained $4 \mu$ DNA template, 1x TaqMan Universal Mastermix II (Life Technologies), 18 pmol each primer (CInnerFor and CInnerRev; see chapter 2 for nucleotide

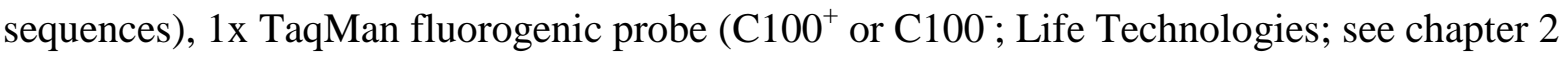
for nucleotide sequences), $10 \mu \mathrm{g}$ BSA (Sigma-Aldrich) and deionised sterile water to a total volume of $20 \mu \mathrm{l}$. Thermal cycling involved an initial $10 \mathrm{~min}, 95^{\circ} \mathrm{C}$ denaturation step followed by 40 cycles of 15 seconds at $95^{\circ} \mathrm{C}$ and $1 \mathrm{~min}$ at $60{ }^{\circ} \mathrm{C}$ (Applied Biosystems StepOne qPCR instrument; Life Technologies). DNA template solutions generated from bulk-cell Symbiodinium samples were run in duplicate with a template-free control reaction included for each probe type. $\mathrm{C}_{\mathrm{t}}$ values were determined as the cycle at which the change in fluorescence was significantly different to the background level $\left(\Delta \mathrm{R}_{\mathrm{n}}=0.05\right.$; obtained using the instrument's built-in algorithm). Reactions featuring $C_{t}$ values below the intercepts of the previously-generated standard curves (36.873 and 37.119 for $\mathrm{C} 100^{+}$and $\mathrm{C}^{-100^{-}}$assays, respectively; see Appendix $B$ ) and sufficiently low standard deviations $(<0.5)$ were included in the analysis. The total number of ITS 2 copies in each sample $\left(\mathrm{C}_{\text {TOTAL }}\right)$ was estimated as the sum of the C100 copy number and the number of Symbiodinium clade C ITS2 sequences other than type $\mathrm{C} 100\left(\mathrm{C}_{\mathrm{C} 100}\right.$ and $\mathrm{C}_{\mathrm{C} 100}{ }^{-}$, respectively). The proportion of total Symbiodinium clade $\mathrm{C}$ sequences that were of type $\mathrm{C} 100$ was expressed as the bulk-cell $\mathrm{C}_{\mathrm{C} 100}$ : $\mathrm{C}_{\text {TOTAL }}$ ratio. This ratio were subsequently converted to the proportional abundance of genetically heterogeneous cells $\left(S_{\mathrm{H}}: S_{\mathrm{T}}\right)$ using the polynomial equation developed in chapter $3(\mathrm{y}=-$ $3.4484 \mathrm{x}^{2}+3.3604 \mathrm{x}$; where $\mathrm{y}=S_{\mathrm{H}}: S_{\mathrm{T}}$ and $\left.\mathrm{x}=\mathrm{C}_{\mathrm{C} 100}: \mathrm{C}_{\mathrm{TOTAL}}\right)$.

\subsubsection{Statistical analysis}

Mortality rates were compared between sites (North Bay and The Arch) and transplant times (195 d and 374 d) using a three-way Pearson's chi-squared cross-tabulation test. For corals transplanted at The Arch, mortality rates were further assessed after $195 \mathrm{~d}$ using a binomial logistic regression model, with the proportional abundance of genetically heterogeneous Symbiodinium cells $\left(S_{\mathrm{H}}: S_{\mathrm{T}}\right)$ as a putative explanatory variable and mortality as the binary response. Initial dry weight (in g) was added as a nuisance covariate and given first opportunity to explain variance within the model, in order to control the type II error rate (see 
Quinn \& Keough 2002). Variation in compounding growth rates $\left(G\right.$; in $\left.\% \mathrm{~d}^{-1}\right)$ and $S_{\mathrm{H}}: S_{\mathrm{T}}$ were assessed using blocked MANCOVA, with Transplant site and Time as factors (fixed-effects; each with two levels), original $S_{\mathrm{H}}: S_{\mathrm{T}}$ as a covariate, and Original site as a block variable. In all cases, normality and homoscedasticity were assessed using Q-Q plots, and Box's and Levene's tests of variance equality. All statistical analyses were carried out using SPSS Statistics v20 (IBM). 


\subsection{Results}

\subsubsection{Coral mortality}

Coral mortality varied according to transplant-site and time since transplantation, but not as a function of the proportional abundance of genetically heterogeneous Symbiodinium cells $\left(S_{\mathrm{H}}: S_{\mathrm{T}}\right)$. Mortality was consistently higher at The Arch than at North Bay $\left(\chi^{2}{ }_{1}=6.656\right.$ and 30.313; $\mathrm{p}=0.010$ and $<0.001$, after $195 \mathrm{~d}$ and $374 \mathrm{~d}$, respectively). A further partial association existed between mortality and time since transplantation, with corals at The Arch experiencing higher mortality rates during the final $179 \mathrm{~d}$ of deployment $\left(\chi^{2}{ }_{1}=9.87 ; \mathrm{p}=\right.$ 0.002). Of the 60 coral fragments deployed at The Arch, 11 perished during the first $195 \mathrm{~d}$, and of the 29 that remained after the first retrieval only seven survived to the end of the 374-d experiment. Conversely, mortality was consistently low at North Bay, with only one of the 60 coral fragments perishing during the first six months, and four more during the subsequent six-month period $\left(\chi_{1}^{2}=0.539 ; \mathrm{p}=0.463\right.$ for $195 \mathrm{~d}$ versus $374 \mathrm{~d}$ comparison $)$. This prevented assessment of the association between $S_{\mathrm{H}}: S_{\mathrm{T}}$ and mortality at North Bay, due to a lack of balance in the binomial logistic regression model. Indeed, this assessment could only be reliably carried out at The Arch after the first retrieval round (195 d since transplantation) due to the low number of surviving fragments at this site after $374 \mathrm{~d}$. High mortality in transplanted corals at The Arch was consistent with the established colonies at this site, many of which perished during the particularly cold winter of 2012 (see chapter 3, Figure 3.3), and were overgrown with macroalgae by the end of the experiment. By contrast, very few of the transplanted corals at North Bay perished, and no noticeable mortality occurred among the established $P$. damicornis colonies at this site over the course of the experiment.

The initial dry weight of transplanted coral fragments emerged as a significant factor in determining the likelihood of mortality, with smaller fragments showing a reduced likelihood of survival. A 1.37-fold increase in survival likelihood was associated with a $1 \mathrm{~g}$ increase in dry weight (Wald test statistic with one degree of freedom $=4.076 ; \mathrm{p}=0.043$ ). After accounting for site- and size-related factors, no association was detected between $S_{\mathrm{H}}: S_{\mathrm{T}}$ and mortality (binomial logistic regression, $\chi_{1}^{2}=0.241 ; p=0.632$; Figure 5.2). 


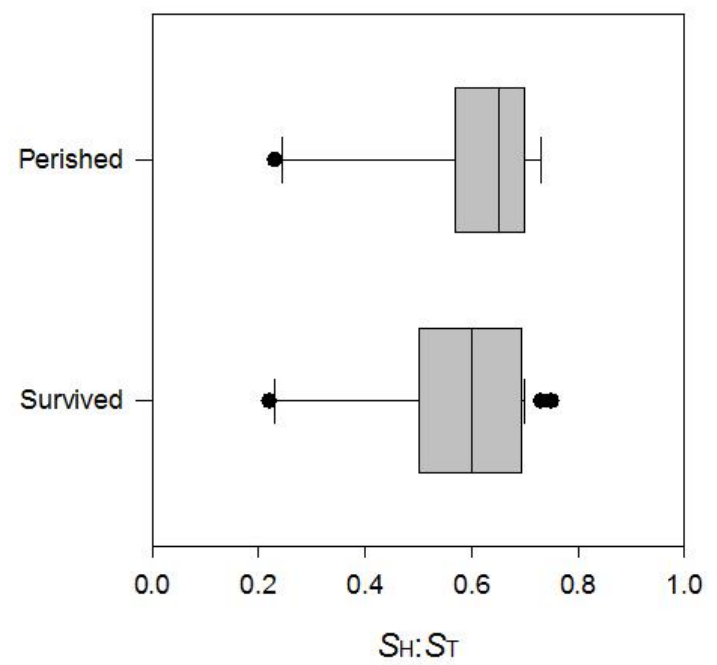

Figure 5.2 Effect of putative Symbiodinium hybrids on coral mortality

Boxes show the median and interquartile range in the proportional abundance of genetically heterogeneous Symbiodinium cells in corals that perished $(n=11)$ or survived $(n=49)$ after $195 \mathrm{~d}$ of deployment at The Arch. Closed circles represent $10^{\text {th }} / 90^{\text {th }}$ percentile outliers. Mortality rates were not significantly affected by $S_{\mathrm{H}}: S_{\mathrm{T}}(\mathrm{p}=0.632)$.

\subsubsection{Physical response to transplantation}

Surviving coral fragments from both sites exhibited high variability in growth, and minor changes in the proportional abundance of genetically heterogeneous Symbiodinium cells. A significant interaction between Transplant site and Time was evident in determining the combined physical response $\left(G\right.$ and $S_{\mathrm{H}}: S_{\mathrm{T}}$; MANCOVA $\mathrm{F}_{2,75}=4.337$; omnibus p-value $=$ 0.017; Table 5.1). Subsequent univariate testing revealed the Transplant site $\mathrm{x}$ Time interaction applied only to coral growth $\left(\mathrm{F}_{1,76}=8.129\right.$ and $0.510 ; \mathrm{p}=0.006$ and 0.477 for $G$ and $S_{\mathrm{H}}: S_{\mathrm{T}}$, respectively; Table 5.2). Simple-effects tests for coral growth at each retrieval time-point revealed that differences between the two sites occurred only during the final 179 $d$ (during which the winter thermal minimum occurred; $F_{1,77}=0.183, p=0.670$ for $195-d$ and $\mathrm{F}_{1,77}=10.761, \mathrm{p}=0.002$ for 374-d transplant time; Table 5.3 and Table 5.4, respectively). Average ( \pm S.E.) daily growth rates over the first $195 \mathrm{~d}$ were $1.755 \times 10^{-3} \pm 0.119 \times 10^{-3} \% \mathrm{~d}^{-1}$ for both sites, and those over the entire 374-d duration were $0.859 \times 10^{-3} \pm 0.374 \times 10^{-3} \% \mathrm{~d}^{-1}$ at the Arch and $1.902 \times 10^{-3} \pm 0.160 \times 10^{-3} \% \mathrm{~d}^{-1}$ at North Bay. The interpolated growth rates during the final $179 \mathrm{~d}$ were therefore $-0.133 \times 10^{-3} \pm 0.780 \times 10^{-3} \% \mathrm{~d}^{-1}$ at The Arch and 2.044 $\times 10^{-3} \pm 0.253 \times 10^{-3} \% \mathrm{~d}^{-1}$ at North Bay (Figure 5.3a). The highest growth rate by a single coral fragment was recorded at North Bay, with an increase in dry weight from $8.65 \mathrm{~g}$ to $38.83 \mathrm{~g}$ in $374 \mathrm{~d}$, representing a compounding growth rate of $4.02 \times 10^{-3} \% \mathrm{~d}^{-1}$. Of 84 surviving colonies in total, three recorded net skeletal erosion over the 374-d period, all of which were deployed at The Arch. 


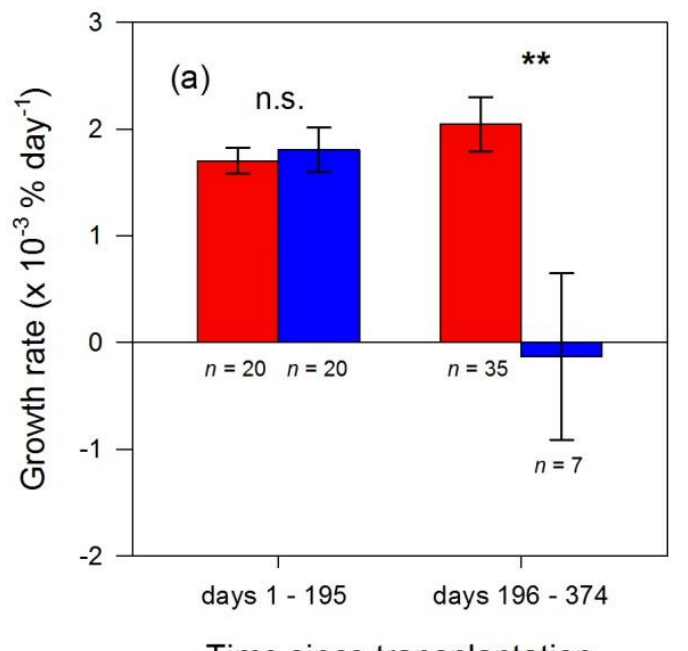

Time since transplantation

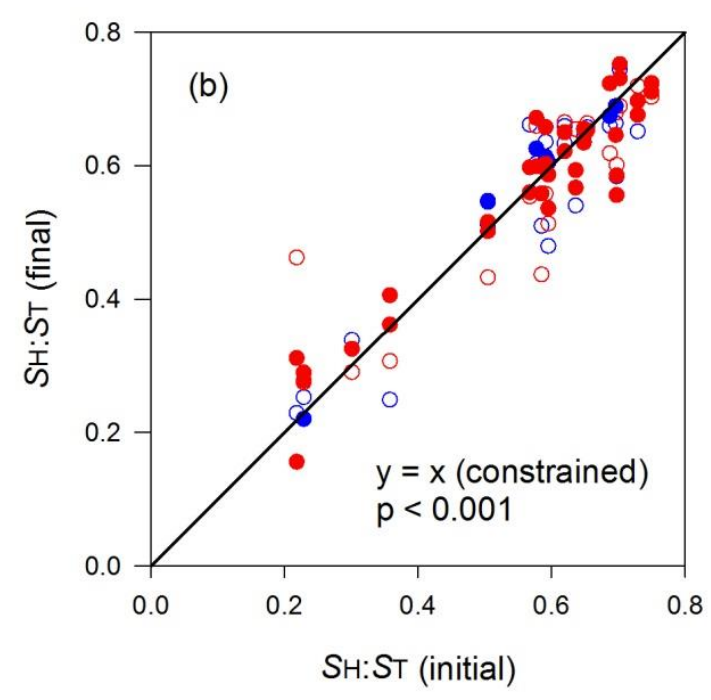

Figure 5.3 Physical responses of $\boldsymbol{P}$. damicornis colonies following transplantation

Coral fragments showed a variety of responses to transplantation, with (a) high variation in growth rates between the transplant sites of North Bay and The Arch (shown in red and blue, respectively; double asterisk denotes a p-value of less than 0.01); and (b) minor temporal changes in the proportional abundance of genetically heterogeneous Symbiodinium cells. Changes in $S_{\mathrm{H}}: S_{\mathrm{T}}$ occurred independently of transplant site and transplant time (shown in red and blue for North Bay and The Arch, and open and closed circles for 195-d and 374-d, respectively). A relatively high level of temporal variation in $S_{\mathrm{H}}: S_{\mathrm{T}}$ was detected between ramets of the same parent colony (16.7\% residual variance component), compared with an earlier longitudinal analysis of established coral colonies (4\% residual variance component; see chapter 3).

An intrinsically higher proportional abundance of genetically heterogeneous Symbiodinium cells in $P$. damicornis colonies at The Arch (see chapter 3) was evident in a significant effect of Original site (i.e. 'block' effect; mean $S_{\mathrm{H}}: S_{\mathrm{T}}=0.583$ and 0.551 and The Arch and North Bay, respectively; $\mathrm{F}_{1,78}=4.053 ; \mathrm{p}=0.048$; Table 5.2). However, neither Transplant site nor Time had a significant influence on the $S_{\mathrm{H}}: S_{\mathrm{T}}$ ratio measured upon retrieval $\left(\mathrm{F}_{1,76}=0.046\right.$ and $1.561, \mathrm{p}=0.830$ and 0.215 , respectively; Table 5.2). The proportional abundance of homogeneous and heterogeneous Symbiodinium cells did not change deterministically throughout the course of the experiment, with the initial $S_{\mathrm{H}}: S_{\mathrm{T}}$ (measured from the parent colony prior to transplantation) emerging as a strong predictor of final $S_{\mathrm{H}}: S_{\mathrm{T}}\left(\mathrm{F}_{1,76}=486.576\right.$; $\mathrm{p}<0.001$; Table 5.2). As such, when the initial and final $S_{\mathrm{H}}: S_{\mathrm{T}}$ ratios were analysed using a before and after paired-differences t-test, no significant change in $S_{\mathrm{H}}: S_{\mathrm{T}}$ was detected $\left(\mathrm{t}_{81}=\right.$ $1.112 ; \mathrm{p}=0.269 ;$ Figure 5.3b). 
Table 5.1 MANCOVA for coral growth and temporal changes in the proportional abundance of putative Symbiodinium hybrids

\begin{tabular}{llllll}
\hline Source & Pillai's Trace & Hypothesis df & Error df & $\boldsymbol{F}$ & $\boldsymbol{P}$ \\
\hline Initial $\boldsymbol{S}_{\mathrm{H}}: \boldsymbol{S}_{\mathrm{T}}$ (covariate) & 0.865 & 2 & 75 & 240.094 & $<0.001$ \\
Original site (block) & 0.052 & 2 & 75 & 2.076 & 0.133 \\
Transplant site & 0.068 & 2 & 75 & 2.729 & 0.072 \\
Time & 0.063 & 2 & 75 & 2.511 & 0.088 \\
Transplant site x Time & 0.104 & 2 & 75 & 4.337 & 0.017 \\
\hline
\end{tabular}

Table 5.2 Univariate ANCOVA for coral growth and temporal changes in the proportional abundance of putative Symbiodinium hybrids

\begin{tabular}{lllllll}
\hline Source & Variable & df & SS & MS & $\boldsymbol{F}$ & $\boldsymbol{P}$ \\
\hline Initial $\boldsymbol{S}_{\mathrm{H}}: \boldsymbol{S}_{\mathrm{T}}$ (covariate) & Growth & 1 & 0.451 & 0.451 & 0.766 & 0.384 \\
& $S_{\mathrm{H}}: S_{\mathrm{T}}$ & 1 & 1.532 & 1.532 & 486.576 & $<0.001$ \\
Original site (block) & Growth & 1 & 0.062 & 0.062 & 0.105 & 0.746 \\
& $S_{\mathrm{H}}: S_{\mathrm{T}}$ & 1 & 0.013 & 0.013 & 4.053 & 0.048 \\
Transplant site & Growth & 1 & 3.205 & 3.205 & 5.443 & 0.022 \\
& $S_{\mathrm{H}}: S_{\mathrm{T}}$ & 1 & $<0.001$ & $<0.001$ & 0.046 & 0.830 \\
Time & Growth & 1 & 1.981 & 1.981 & 3.364 & 0.071 \\
\multirow{5}{*}{ Transplant site x Time } & $S_{\mathrm{H}}: S_{\mathrm{T}}$ & 1 & 0.005 & 0.005 & 1.561 & 0.215 \\
& Growth & 1 & 4.786 & 4.786 & 8.129 & 0.006 \\
Error & $S_{\mathrm{H}}: S_{\mathrm{T}}$ & 1 & 0.002 & 0.002 & 0.510 & 0.477 \\
& Growth & 76 & 44.748 & 0.589 & & \\
\hline & $S_{\mathrm{H}}: S_{\mathrm{T}}$ & 76 & 0.239 & 0.003 & & \\
\hline
\end{tabular}

Table 5.3 Simple-effects analysis of coral growth over 195 days

\begin{tabular}{llllll}
\hline Source & df & SS & MS & $\boldsymbol{F}$ & $\boldsymbol{P}$ \\
\hline Transplant site & 1 & 0.107 & 0.107 & 0.180 & 0.673 \\
Error & 76 & 45.199 & 0.595 & & \\
\hline
\end{tabular}

Table 5.4 Simple-effects analysis of coral growth over 374 days

\begin{tabular}{llllll}
\hline Source & Df & SS & MS & $\boldsymbol{F}$ & $\boldsymbol{P}$ \\
\hline Transplant site & 1 & 6.317 & 6.317 & 10.62 & 0.002 \\
Error & 76 & 45.199 & 0.595 & & \\
\hline
\end{tabular}




\subsection{Discussion}

The photosynthetic performance of the symbiont is often a strong determinant of coral fitness; however the host's physiology and behaviour can uncouple this association (reviewed in Baird et al. 2008). Similarly, the performance of the symbiont may not necessarily reflect its mutualistic scope (Stat et al. 2008b). This study aimed to determine whether physiological differences observed between genetically homogeneous and heterogeneous Symbiodinium cells (see chapter 4) affect the growth and survival of their coral hosts in the natural reef setting. Colonies were reciprocally transplanted between two distinct reef habitats, an inner lagoon site exposed to pronounced thermal peaks, and a well-flushed site situated at the transition zone between coral- and macroalgal-dominated communities. The proportional abundance of putative hybrid symbionts did not influence coral mortality or calcification, nor did it change in response to habitat modification. Instead, coral fitness appeared to be predominantly driven by local environmental factors, with the inner-lagoon site providing a more suitable habitat for coral growth and survival.

\subsubsection{The influence of putative Symbiodinium hybrids on coral fitness}

Coral calcification and mortality were not affected by the proportional abundance of genetically heterogeneous Symbiodinium cells. This was inconsistent with the comparatively poor photosynthetic performance shown by these symbionts in a controlled setting (see chapter 4). This discrepancy may have arisen via several processes. First, conditions simulated in an experimental setting are not representative of those experienced on the reef. While the earlier assessment of photosynthetic performance was carried out using stable temperatures approximating the summer average and absolute thermal maximum experienced within the Lord Howe Island lagoon $\left(25\right.$ and $29^{\circ} \mathrm{C}$, respectively; see chapter 4$)$, pronounced fluctuations in temperature and light characterise the LHI reef environment (Veron \& Done 1979; Harrison et al. 2011). Coral fragments were also exposed to temperatures that were consistently lower than $25{ }^{\circ} \mathrm{C}$ throughout the course of this study. While it is presently unknown how heterogeneous Symbiodinium cells perform under cold-stress, their spatial distribution pattern indicates a possible adaptation to cooler environments (see chapter 3). The extreme thermal minimum experienced at LHI plays an important role in determining range boundaries of corals and other reef fauna at this site (Veron \& Done 1979), and cold stress is a known causal factor underlying coral bleaching and symbiont community change 
in Pocillopora colonies in the Gulf of California (LaJeunesse et al. 2010b). Cold-adaptation or other factors may have equalized the disparity in photosynthetic performance between homogeneous and heterogeneous Symbiodinium cells in their natural setting. Second, the biochemical and/or behavioural activity of the coral host may have compensated for the poor photosynthetic performance of its symbionts. Corals may employ various strategies to ensure that their metabolic requirements are met, with some species even switching to a heterotrophic feeding mode in the absence of symbiont-derived compounds (Grottoli et al. 2006). Indeed, P. damicornis colonies hosting predominantly heterogeneous Symbiodinium cells showed marginal increases in protein biomass, a response consistent with this mode of nutrient assimilation (see chapter 4). Finally, a reduction in photosynthetic performance may be offset if a symbiont occupies a particularly beneficial position along the parasitismmutualism continuum. In the majority of cases, coral fitness varies according to the degree of host-symbiont co-operation rather than host- or symbiont factors in isolation (Bhagooli \& Hidaka 2003; Abrego et al. 2008). In particular, the quality and quantity of translocated photosynthate may differ widely among symbiont taxa, with important implications for host fitness (Little et al. 2004; Loram et al. 2007b; Stat et al. 2008b; Cantin et al. 2009; Jones \& Berkelmans 2010; Starzak et al. 2014). For example, the clade A symbionts of the coral Acropora cytherea released significantly less carbon compared with those of clade $\mathrm{C}$, with colonies hosting the former showing a corresponding increase in disease risk (Stat et al. 2008b). The uncoupling of symbiont performance and host fitness observed in this study suggests that some beneficial feature of putative hybrid Symbiodinium cells may warrant their persistence in the $P$. damicornis symbiont consortium. This may explain their widespread abundance on the LHI reef, and the lack of temporal variation within colonies (see chapter 3 and next section). Identification of this 'redeeming' characteristic presents an interesting area for future investigation.

\subsubsection{Changes in symbiont ratios following transplantation}

Minor temporal changes in ratios of genetically homogeneous and heterogeneous symbionts were apparently random, and not attributable to environmental factors. Corals that transmit their symbionts vertically (such as $P$. damicornis) generally show reduced symbiotic flexibility (Thornhill et al. 2006a); however this contrasted with several previous transplant studies that show deterministic symbiont shuffling in both horizontal- and vertical- 
transmitting species (Baker 2001; Toller et al. 2001b; Berkelmans \& van Oppen 2006). For example, colonies of the vertically-transmitting coral Porites astreoides underwent a change in dominant symbiont from clade $\mathrm{C}$ to clade $\mathrm{A}$ when transplanted to a shallower habitat on a Caribbean reef (Baker 2001), a response that ostensibly led to an improvement in thermal tolerance. While no deterministic symbiont shuffling was observed in the present study, symbiont ratios were temporally variable in transplanted corals compared with those established colonies (see chapter 3). This increased random variation in $S_{\mathrm{H}}: S_{\mathrm{T}}$ may have arisen from the fragmentation process, causing stochastic drift in the proportional abundance as a result of a 'population bottleneck'. Indeed, the effect may have been compounded by partial bleaching experienced by fragments following transplantation. The sudden exposure of previously-shaded branch areas to excess irradiance resulted in visible paling in all transplanted colonies (including the transplanted controls) on the North Bay reef approximately one week after transplantation, while no such bleaching was apparent in the parent colonies (pers. obs; a follow-up visual assessment of fragments and parent colonies could not be carried out at The Arch, due to difficulties accessing this site). These findings support the assertion made in chapter 3: that the symbiont consortium of $P$. damicornis is determined during early ontogeny and/or bleaching, with genetically homogeneous and heterogeneous Symbiodinium cells apparently not subject to intra-specific competition or selective expulsion by the host.

\subsubsection{Methodological considerations}

While symbiont performance was apparently uncoupled from host fitness in this study, several factors may have prevented the detection of underlying patterns. Stochastic events such as abiotic disturbance, interspecific competition, predation and disease can substantially increase the variability in coral growth and survival on the reef (Correa \& Baker 2011). Gaining reliable estimates of fitness in the field is hampered by these processes, particularly when sample sizes are small (Garrison \& Ward 2012). Measuring the influence of putative Symbiodinium hybrids on host survival was further hindered by the inability to retrieve coral fragments at a time when approximately equal numbers had perished and survived (thus maximizing statistical power; Quinn \& Keough 2002). Future assessments of coral- and symbiont-fitness may benefit from employing larger sample sizes and attempting to control for the many sources of extraneous variation encountered in the natural setting. Moreover, 
fitness is a measure of an individual's genetic contribution to subsequent generations (Maynard Smith 1998). Estimates of performance, growth and survivorship can give an indication of fitness, but may not provide an absolute measure (Arnold 1983). For example, in a comparison of the effects of different symbiont types on the fitness of the anemone Aiptasia sp., homologous algae stimulated increased biomass while heterologous algae prompted an increase in reproductive output (Kinzie \& Chee 1979). Further attempts to establish a link between symbiont hybridization and coral fitness should therefore also incorporate measures of reproductive success.

\subsubsection{Conclusion}

The relative abundance of genetically heterogeneous Symbiodinium cells did not appreciably affect the growth or survival of transplanted corals in the field, despite the impaired photosynthetic function of these symbionts at elevated temperature. These findings highlight the fact that a coral's fitness may be uncoupled from the photosynthetic performance of its symbiont, through processes mediated by either or both symbiotic partners. An apparent lack of negative long-term effects associated with hosting putative Symbiodinium hybrids may help to explain their temporal stability within colonies and their widespread abundance on the Lord Howe Island reef. Further research into the mutualistic scope of hybrid Symbiodinium cells may reveal concealed benefits, such as adaptation to extreme conditions or reduced virulence. Establishing the implications of symbiont hybridization for coral fitness will ultimately help to evaluate the evolutionary significance of this process in a rapidly changing environment. 


\section{Chapter 6: General discussion}

The preceding data chapters provide several lines of indirect evidence for hybridization in symbiotic dinoflagellates. These include: (1) the intra-genomic co-dominance of divergent ITS2 sequences; (2) significant genetic clustering of heterogeneous and homogeneous symbionts; (3); the coexistence of genetically heterogeneous symbionts with both putative progenitors; (4) thermal niche partitioning, consistent with the diversification of hybrids into 'extreme' habitats (i.e. the coral-macroalgal transition zone); (5) differences in morphology and physiology, with putative hybrids $50 \%$ larger by volume and showing a two-fold increase in chlorophyll $c_{2}: a$; and (6) disparities in photosynthetic function, consistent with the deleterious segregation of unlinked genes in haploid hybrids ('hybrid breakdown'). A review of the literature also indicates that hybridization may have occurred among other Symbiodinium taxa. In particular, this hypothesis is advanced by emerging evidence of sexual reproduction in symbiotic dinoflagellates (Baillie et al. 1998, 2000; LaJeunesse 2001; Santos et al. 2003b; Pettay et al. 2011; Thornhill et al. 2013a; Chi et al. 2014; Baums et al. 2014), a maximum divergence threshold of around 5-10\% ITS dissimilarity for viable hybridization in other dinoflagellates, diatoms and haplontic green algae (Coleman et al. 1994; Edvardsen et al. 2003; Vanormelingen et al. 2008), and the recent finding of diploidy and allelic additivity in the heat-tolerant species Symbiodinium trenchii (LaJeunesse et al. 2014). While evidence remains indirect, these results collectively provide a compelling argument that hybridization is a naturally occurring phenomenon in the Symbiodinium genus.

\subsubsection{Confirming hybridization in Symbiodinium}

The findings of this study highlight the need for direct evidence of hybridization in Symbiodinium. This is likely best accomplished through continued attempts to induce the sexual life-cycle within and between Symbiodinium lineages in culture. While putative gametes have been observed by several authors (Freudenthal 1962; Taylor 1973, 1974; Fitt \& Trench 1983), life-cycle stages are often misinterpreted in dinoflagellates (Elbrächter 2003). This is particularly true of gametogenesis, which often involves simple vegetative division (hologamy; Pfiester \& Anderson 1987). Conclusive evidence of this process has continued to evade researchers, presenting a major challenge to determining the evolutionary significance 
of recombination in the Symbiodinium genus. Yet cryptic sexual life cycles have been increasingly exposed in free-living 'asexual' dinoflagellates (e.g. Parrow \& Burkholder $2003 \mathrm{a} ; \mathrm{b}, 2004)$, with the simulation of nutrient stress often proving the necessary stimulant (Turpin et al. 1978; Anderson et al. 1984; Chesnick \& Cox 1987; Figueroa et al. 2007, 2011). Experimental crossing of closely related types may also help to induce the sexual life-cycle, since mating incompatibilities often prevent isoclonal dinoflagellates from conjugating (Destombe \& Cembella 1990; Parrow \& Burkholder 2003a). The experimental stimulation of sexual reproduction in Symbiodinium would constitute an important scientific breakthrough, with potentially major implications for coral adaptation in a changing climate. As such, research in this area should be a high priority.

In the absence of direct evidence of sexual reproduction and hybridization in culture, convincing evidence of these processes may be obtained from genomic analysis. A preliminary objective is to determine the multi-locus genotype (MLG) of each clone within a symbiont assemblage. Yet MLG techniques require DNA from individual organisms or isoclonal cell-lines (Santos et al. 2003b). While some corals appear to harbour a single symbiont genotype (Goulet \& Coffroth 2003a; b; Pettay \& LaJeunesse 2007; Thornhill et al. 2009; Andras et al. 2011; Pettay et al. 2011; Baums et al. 2014), and thus effectively provide a 'culture vessel' of vegetative growth (LaJeunesse et al. 2012), this is clearly not the case for many species (e.g. Rowan \& Knowlton 1995; Stat et al. 2011; Green et al. 2014). Indeed, the results presented in this thesis suggest that the $P$. damicornis symbiont consortium can consist of at least three (and possibly many more) clonal genotypes. These symbionts do not readily survive in culture (S. Pontasch, VUW, pers. comm.), a trait characteristic of many vertically-transmitted Symbiodinium lineages (Krueger \& Gates 2012). However, a solution may be found in whole genome amplification (WGA). Recent developments in WGA using the bacteriophage $\Phi 29$ DNA polymerase (Dean et al. 2002) show promising results for obtaining MLG data from single cells. For example, WGA facilitated the successful amplification of 20 different loci from a single human lymphocyte (Handyside et al. 2004). Application of this technology to the unicellular organisms of the coral holobiont could greatly improve the resolution of population genetic studies, and thus help to identify the genealogical origins of non-culturable Symbiodinium cells.

The inability to culture Symbiodinium C100, C109 or their putative hybrids necessitated genotyping by single-cell PCR (scPCR). While this method enabled the unambiguous distinction of intra- and inter-genomic sequence variation, it is currently restricted to 
targeting a single, high-copy-number marker from each Symbiodinium cell. The ITS2 locus was chosen for its wide utility, phylogenetic sensitivity and amenability to scPCR (since it occurs in multiple copies per genome). However, its multi-copy nature also renders it subject to intra-genomic variation arising from the incomplete concerted evolution of ancestral polymorphisms, presenting an unavoidable limitation to this study. Despite its drawbacks, the ITS2 can reveal important evolutionary processes, including hybridization, hybrid speciation, reticulate evolution and adaptive radiation (Álvarez \& Wendel 2003), particularly when used in conjunction with morphological, physiological and ecological data (Peterson et al. 2004; Vriesendorp \& Bakker 2005). DNA meta-barcoding now offers semi-quantitative estimates of ITS2 copy numbers and other genetic markers at the subclade level through the generation of high-throughput sequence data (Green et al. 2014). Applying this powerful molecular tool to the individual symbiont cell could facilitate large-scale assessments of intra-genomic variation and inter-genomic diversity, helping to establish the incidence of hybridization and solve the complex puzzle of Symbiodinium evolution.

\subsubsection{Implications of Symbiodinium hybridization}

Over the past two decades or so, a prolific research effort has sought to predict the future of coral reefs in a changing climate. While symbiont shuffling has been identified as an important acclimatory mechanism offering short-term relief from environmental stress, the so-called 'adaptive bleaching' response does not appear to be a heritable modification, and hence its potential benefits are ostensibly limited. Understanding the means by which corals and their symbionts adapt sensu stricto is therefore of paramount importance. This will require a major shift in focus towards the evolutionary effects of somatic mutation accumulation and meiotic recombination within and between lineages. In particular, symbiont hybridization has the potential to offer rapid heritable modification to the coral holobiont, and hence play a critical role in its evolution in a shifting adaptive landscape. The putative emergence of this phenomenon prompts several questions about its ecological and evolutionary significance: When, where and how does symbiont hybridization occur? What are the immediate mutualistic effects and long-term adaptive consequences for the coral host? Could symbiont hybridization be applied in future attempts to improve reef resilience? These considerations are discussed in detail below and accompanied by suggested areas for future research. 


\subsubsection{When, where and how does hybridization occur?}

For hybridization to contribute to adaptation, reproductive barriers must initially be overcome. Establishing the timing, location and divergence thresholds for sexual reproduction in Symbiodinium are therefore important objectives for understanding the evolutionary significance of hybridization in this genus. While the ITS2 admixture depicted in chapter 2 suggests that frequent recombination events may have produced several hybrid genotypes (see Figure 2.4), the absence of MLG data prevents the identification of individual hybrid classes. On one hand, a single ancient hybridization event may have given rise to a divergent, reproductively isolated hybrid lineage whose genome is in various stages of concerted evolution. On the other hand, hybridization and backcrossing may occur on a regular basis, with the occasional fit recombinant undergoing asexual proliferation. The findings of several fine-scale population-level studies indicate that sexual reproduction occurs frequently within Symbiodinium lineages (e.g. Santos et al. 2003b; Pettay et al. 2011; Thornhill et al. 2013a; LaJeunesse et al. 2014; Baums et al. 2014). However, none of these studies detected the recombination of alleles between lineages, indicating that if successful hybridization does occur in this group it is likely an isolated and/or infrequent occurrence. Competition with parental taxa and 'transgressive segregation' (the emergence of extreme hybrid phenotypes) often confine hybrids to the range margins of parental taxa (Arnold 1997; Rieseberg 1997; Rieseberg et al. 2003), and hybrid symbionts of terrestrial plants show a similar distribution pattern (e.g. Barrett et al. 2007; Hamilton et al. 2009). Therefore, Symbiodinium hybridization may occur exclusively in marginal coral reef habitats. Yet hybridization is stimulated both by disturbance (Anderson \& Stebbins 1954) and species range shifts (Hoffmann \& Sgrò 2011); hence an increase in frequency may occur as conditions continue to deteriorate.

Ancient hybridization: Hybridization may not necessarily occur frequently to have important evolutionary effects. The chance occurrence of a single fit hybrid recombinant can give rise to a novel lineage, provided that the adaptive allele combination can stabilize and that reproductive isolation is upheld (Grant 1981; Figure 6.1). This mode of speciation has been implicated in major adaptive radiations, particularly when hybrids occupy vacant niches arising from environmental upheaval (Anderson \& Stebbins 1954; Seehausen 2004; Bell \& Travis 2005; Grant et al. 2005; Mallet 2007). Hybrid speciation is commonly associated with 
allopolyploidy (when an $\mathrm{F}_{1}$ hybrid inherits a set of chromosomes from each parent), since chromosome doubling can offer instant genetic isolation from both progenitors (reviewed in Mallet 2007; Abbott et al. 2013). Yet an increasing number of cases of homoploid hybrid speciation have been identified, particularly when hybrids diversify into extreme habitats via transgressive segregation (Rieseberg 1997; Buerkle et al. 2000; Rieseberg et al. 2003; Gompert et al. 2006). For example, in the wild sunflower genus Helianthus, hybrid species have diversified into deserts and salt marshes (Rieseberg et al. 2003), and in the butterfly genus Lycaeides, a homoploid hybrid species of L. melissa and L. idas occurs exclusively in extreme alpine habitats (Gompert et al. 2006). Hybrid diversification and speciation are also prevalent in symbiont lineages. A hybrid strain of the poplar rust pathogen Melampsora sp. occurs at the southern range margin of its progenitor Melampsora lini in Australia (Barrett et al. 2007), and in the endophytic fungal symbionts of the grass family Poaceae, hybrid species of the genus Neotyphodium are common in habitats characterised by low moisture and reduced nutrients, in comparison to their Epichloë progenitors (Hamilton et al. 2009).

Hybridization may have played an important role in recent adaptive radiations of symbiotic dinoflagellates. Host-taxon niche diversification can present a powerful diverging force (Thornhill et al. 2013a), and may provide the ecological isolation necessary to reinforce hybrid speciation (Buerkle et al. 2000). The polytomic clade D lineage presents a particularly feasible case of adaptive radiation via hybridization. This group includes at least four species that show adaptation to 'extreme' environmental conditions (Symbiodinium trenchii, S. boreum, S. eurythalpos and S. glynni nomen nudum; LaJeunesse et al. 2014). In particular, several characteristics of $S$. trenchii suggest a possible origin of hybrid speciation. S. trenchii has one of the largest genomes of all symbiotic dinoflagellates (LaJeunesse et al. 2005), it routinely possesses two alleles at microsatellite loci (Pettay \& LaJeunesse 2009; Wham et al. 2011; LaJeunesse et al. 2014), and its ITS2 profile shows a co-dominant pattern diagnostic of both Symbiodinium D1a and S. glynni (LaJeunesse 2002).While polyploidy can spontaneously arise in asexual lineages through abnormal mitotic cell division (resulting in whole-genome duplication), this species also shows ecological traits resembling a hybrid species. For example, $S$. trenchii characteristically inhabits thermally-extreme environments (Berkelmans \& van Oppen 2006; Keshavmurthy et al. 2012) and rapidly colonises bleached corals, aggressively out-competing other symbiont taxa under thermally-stressful conditions (LaJeunesse et al. 2009). 


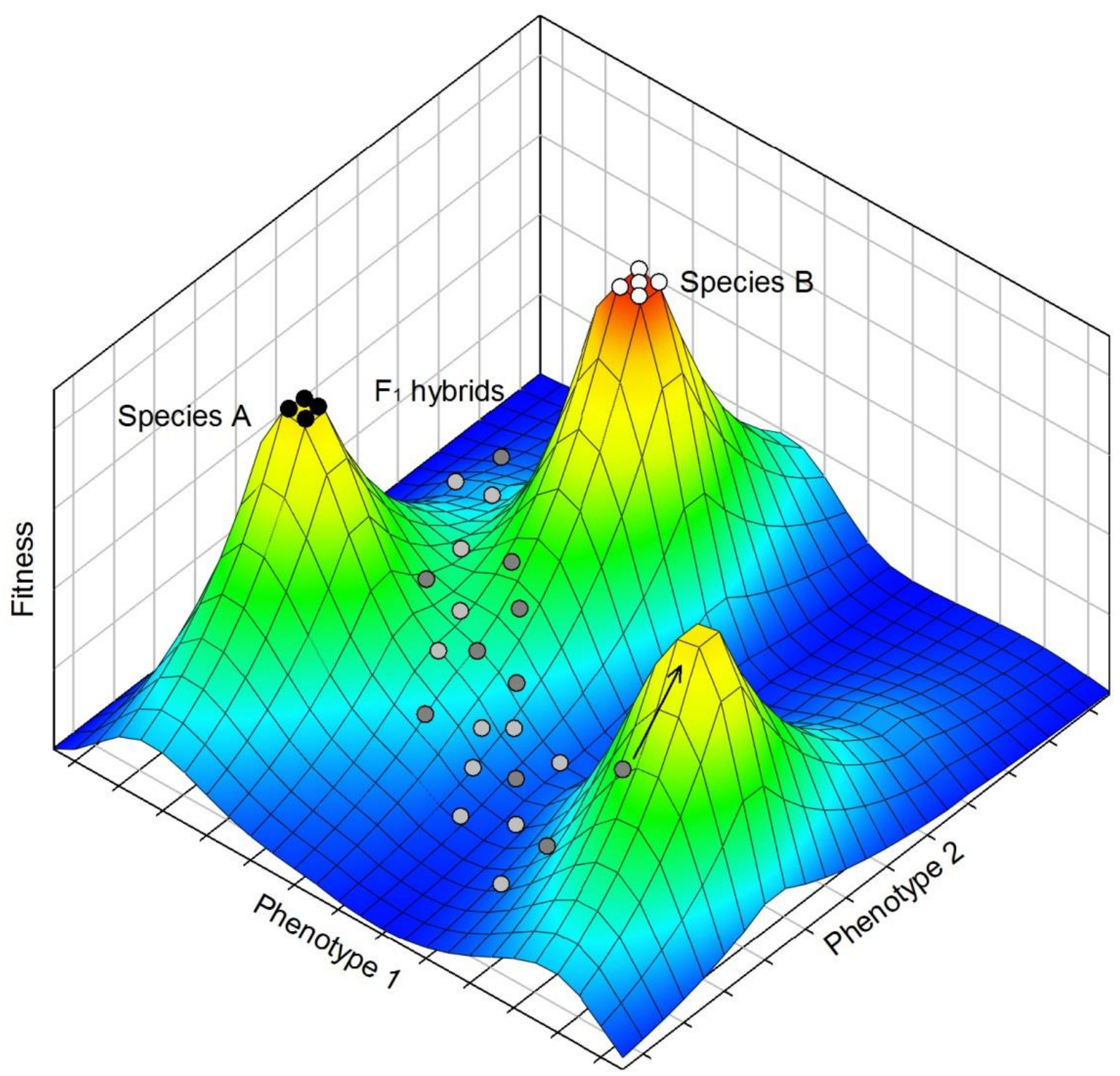

\section{Figure 6.1 Adaptive divergence via hybridization}

Two species, A (black) and B (white) are superimposed on an adaptive landscape alongside $\mathrm{A} \times \mathrm{B}$ hybrids (grey, with many possible haploid genotypes). The plane represents two quantitative phenotypic characters that are subject to 'fitness optima' (shown in a colour gradient). In reality, environmental change causes the landscape to shift continuously, and natural selection simultaneously acts on many phenotypic traits, meaning that the landscape is multi-dimensional (Wright 1932). Hybridization can lead to macro-evolutionary 'jumps'. If a fortunate hybrid recombinant hits an unoccupied adaptive peak, it may exploit the vacant niche, stabilize and undergo hybrid speciation (shown by arrow; adapted from Wright 1932; Simpson 1953; Mallet 2007). 
While S. trenchii presents an obvious candidate hybrid species, several other symbiotic dinoflagellates also warrant further investigation. For example, the C3m ITS2 sequence is codominant with that of C3 in symbionts of the zoanthid Protopalythoa sp. in Hawaii (LaJeunesse et al. 2004); yet C3m aligns with the C1 radiation. This suggests that, either the same ITS2 nucleotide substitution that arose during the divergence of $\mathrm{C} 1$ from the $\mathrm{C} 3$ lineage arose independently during the more recent divergence of $\mathrm{C} 3 \mathrm{~m}$ from the $\mathrm{C} 3$ lineage (i.e. homoplasy), or hybridization has occurred between members of the $\mathrm{C} 1$ and $\mathrm{C} 3$ radiations (LaJeunesse et al. 2005). Could the genetically heterogeneous Symbiodinium C100 × C109 cells represent a divergent, reproductively isolated hybrid species? The diversification of heterogeneous symbionts into a more 'marginal' habitat from that of the putative progenitor (C100) is consistent with hybrid speciation, since niche partitioning is often a necessary precursor for this mode of divergence (Buerkle et al. 2000). However, while morphological, physiological and ecological evidence may allude to hybrid speciation, this process can be notoriously difficult to demonstrate. In order to provide convincing evidence that a species is of hybrid origin, natural selection should favour similar gene combinations in experimental crosses (i.e. synthetic hybrids) under similar environmental conditions (e.g. Rieseberg et al. 2003). Yet even this may be insufficient to unambiguously confirm a species' genealogical origin (Morrell \& Rieseberg 1998).

Frequent hybridization: Another scenario that could explain the genotypic clustering patterns observed in chapter 2 is that Symbiodinium C100 and C109 remain reproductively compatible. Under this scenario, the cluster of genetically heterogeneous Symbiodinium cells may arise from frequent hybridization events, and hybrids may occasionally backcross to the parental types. If this is the case, where and when does hybridization occur? The majority of adult coral colonies appear to host just one or a few Symbiodinium clones, and symbiont reproduction appears to be exclusively asexual when in hospite (LaJeunesse et al. 2012). Here, symbionts are enclosed in membrane-bound vacuoles within the host's gastrodermal cells (see Davy et al. 2012), presumably preventing them from accessing potential mates. Furthermore, theory predicts that hosts should actively limit the reproductive opportunities of the symbionts, in order to prevent symbiotic disruption arising from competition between closely-related individuals (Frank 1996a). It therefore seems unlikely that Symbiodinium sexual reproduction occurs in the multicellular coral host. By contrast, free-living Symbiodinium cells can be highly mobile, showing directed movement towards chemical and physical cues (Fitt 1984; Hollingsworth et al. 2005; Pasternak et al. 2006), and existing in 
high densities in the reef sediment and water column (Coffroth et al. 2006; Littman et al. 2008; Pochon et al. 2010; Takabayashi et al. 2011). The unlimited access to potential mates suggests that the symbionts of horizontally-transmitting corals probably undergo sexual reproduction outside of the host (Trench 1997; but see Baums et al. 2014). However, extensive recombination of alleles has also occurred in S. glynni populations isolated from closely related, vertically-transmitting Pocillopora spp. in the Gulf of California (Pettay et al. 2011). This is clearly at odds with the theory that symbiont sexual reproduction occurs exclusively ex hospite.

Frank (1996a) suggested that symbiotic hosts may derive adaptive benefit from the delineation of symbionts into transmissible germ lineages and non-transmissible somatic lineages. If a portion of a coral's symbionts are sexual, and these symbionts are transmitted to the progeny, then symbiont fusion, plasmogamy and recombination may occur early during the host's ontogenetic development. The ovum of vertically-transmitting corals presents a unique situation where several non-clonal symbionts coexist within a single host cell. Information on the assortment of symbionts into the ovum is scarce (Davy \& Turner 2003; Davy et al. 2012); however Padilla-Gamiño et al. (2012) showed that Hawaiian Montipora capitata colonies release eggs containing between 2300 and 4200 Symbiodinium cells, and many eggs contain distinct symbiont consortia from that of the parent colony. These authors suggested that adaptive diversification may result from the selective assortment of symbionts into gametes. The hypothesis outlined here takes the suggestion of Padilla-Gamiño et al. (2012) a step further, by proposing that under certain conditions, an adaptive advantage may be realized by both partners if symbiont sexual reproduction occurs in synchrony with that of the host. Under this scenario, the onset of symbiont sexual reproduction occurs in the host gamete, zygote and/or developing embryo. Natural selection then acts on a cohort of coral juveniles with a wide range of genotypes and hosting a wide range of recombinant symbionts, thus conferring maximum likelihood of hitting a vacant 'adaptive peak' (see Figure 6.1).

It is not unusual for symbiont sexual reproduction to occur in synchrony with that of the host. For example, the dinoflagellate Peridinium balticum and its membrane-bound endosymbiotic heterokont (Bacillariophyta) undergo concomitant sexual reproduction (Chesnick \& Cox 1987). Indeed, major hybridization events in symbiont lineages appear to track those of their hosts. For example, a hybrid poplar was widely grown in California on account of its resistance to the two rust pathogens that infected its parent species (Melampsora medusa, the parasite of Populus deltoids, and M. occidentalis, the parasite of P. trichocarpa). However, in 
1997 these pathogens crossed to produce the hybrid species Melampsora $\times$ columbiana, which was able to infect the hybrid poplar (Newcombe et al. 2000). In another example, the tall fescue grass Lolium arundinaceum (=Festuca arundinacea) and its fungal endosymbiont Neotyphodium $\times$ coenophialum have each undergone two separate hybridizations, culminating in the combination of three plant genomes and three fungal genomes (Tsai et al. 1994; Humphreys et al. 1995; Schardl \& Craven 2003; Moon et al. 2004).

The possibility that $P$. damicornis and its symbionts have undergone synchronous hybridization presents an interesting topic for investigation. $P$. damicornis undergoes extensive hybridization in nature (Combosch et al. 2008), and shows a predominantly sexual reproductive mode at Lord Howe Island, where it is even known to undergo intergeneric hybridization with Stylophora pistillata (Miller \& Ayre 2004). While sexual reproduction in P. damicornis appears to be more prevalent at marginal reefs, the majority of offspring produced are brooded asexual larvae, and the cryptic simultaneous broadcast-spawning of gametes can be difficult to detect (Combosch \& Vollmer 2013). As such, testing the hypothesis that the reproductive mode of the symbiont is intertwined with that of the host will likely require a population genetics approach, tracking the genealogical origins of both symbiotic partners over several generations. In the interim, the feasibility of this hypothesis may be assessed by evaluating the potential costs and benefits in terms of host fitness.

\subsubsection{How does symbiont hybridization affect coral fitness?}

The occurrence of natural hybridization between symbiotic dinoflagellates may have a major influence on the fitness of the coral host. Symbiont-derived compounds constitute the primary source of energy for reef-building corals (Muscatine 1990; but see Grottoli et al. 2006), and hence even minor disparities in photosynthetic performance can have a profound impact on the host's ability to survive and reproduce (e.g. Cantin et al. 2009). Symbiont hybridization may therefore be detrimental to the host if hybrid breakdown inhibits performance, but similar microbe-associated molecular patterns are inherited (MAMPs; see Davy et al. 2012) and hence the host is unable to recognize deleterious hybrids. Alternatively, symbiont hybridization may benefit host fitness through the instantaneous production of a novel asexual lineage with increased competitive ability (Stebbins 1950) and a 'clean slate' from deleterious mutations. 
The effects of symbiont hybridization on host fitness are highlighted by the example of symbiotic fungi in the genera Epichloë and Neotyphodium. These endophytic ascomycetes infect cool-season grasses of the family Poaceae (subfamily Poöideae), an association that spans the parasitism-mutualism continuum. Most asexual Neotyphodium spp. symbionts are of hybrid origin (Schardl et al. 1994; Tsai et al. 1994; Moon et al. 2004; Hamilton et al. 2009), a process that has been implicated in their recent radiation (Tsai et al. 1994). Hybridization has also been central to the evolution of mutualism in Neotyphodium spp., which are transmitted from parent to offspring and offer a wide range of adaptive benefits to their hosts. For example, repeated hybridization events have led to the accumulation of alkaloid-production genes in the hybrid species Neotyphodium $\times$ coenophialum, which can produce three of the four known classes of endophyte- associated anti-herbivore alkaloids (Clay \& Schardl 2002). Indeed, grasses hosting asexual Neotyphodium hybrids often show increased fitness compared to those that host 'pure' types, including resistance to herbivory and disease, and the ability to survive in extreme environments (Malinowski \& Belesky 2000; Clay \& Schardl 2002; Hamilton et al. 2009; Saari \& Faeth 2012). By contrast, the horizontally-transmitted, sexual progenitor Epichloë sp. can reduce host fitness by 'hijacking' the plant's reproductive structures in order to facilitate spore dispersal (choke disease; Schardl \& Clay 1997). Hybridization may also benefit both symbiotic partners by preventing the accumulation of deleterious mutations in a vertically-transmitted symbiont lineage (i.e. the counteraction of Muller's ratchet; Muller 1964; Felsenstein 1974). The occasional recombination that occurs between Neotyphodium and Epichloë lineages is thought to play a key role in preventing 'ratchet clicks' in mutualistic fungal endophytes (Schardl et al. 1991; Clay \& Schardl 2002; Schardl \& Craven 2003). While fungal hybridization outside the symbiosphere is uncommon, the diverse array of fungal hybrids in vertically-transmitting grasses implies a host role in the outcrossing process (Clay \& Schardl 2002). It is not known whether the host actively selects beneficial asexual hybrid genotypes or the particular ecological niche simply favours hybrids when they occur (Schardl \& Craven 2003; Moon et al. 2004). However, the hybrid fungal endophyte example serves to illustrate the substantial fitness benefits that a host may derive from symbiont hybridization.

The corals that were dominated by putative hybrid symbionts in this study showed significant reductions in overall photosynthetic performance ( $P: R$; see chapter 4). Yet these colonies did not suffer from decreased growth rates or higher mortality in the field (chapter 5). The apparent uncoupling of symbiont photosynthetic performance and host fitness suggests that 
corals may realize compensatory adaptive benefits from hosting hybrid Symbiodinium. The literature on fungal-plant mutualisms suggests that these benefits could include an increased ability to survive in extreme environments, a reduced risk of symbiont virulence, the production of secondary compounds, and the release from mutation accumulation in vertically-transmitted symbiont lineages. This suite of potential adaptive benefits indicates that the coral host may indeed play an active role in the creation of new symbiont lineages.

\subsubsection{What are the evolutionary implications of symbiont hybridization?}

Hybridization can expedite the evolutionary process by providing the raw genetic material necessary for adaptation (Anderson 1949). In spatial areas where related species hybridize frequently ('hybrid zones'), fertile $\mathrm{F}_{1}$ hybrids may 'backcross' with individuals from one or both progenitor populations. The mating of progenitors with progressively later-generation backcross genotypes can lead to the migration of genetic material between lineages (introgression; see Figure 2.1b). Introgressive hybridization can incur profound evolutionary consequences for the recipient population(s). These range from the introduction of 'genetic pollution' to a native lineage (even leading to extinction; reviewed in Rhymer \& Simberloff 1996) to the acquisition of novel genetic diversity, providing a 'fast track' to adaptive evolution (Anderson \& Stebbins 1954; Lewontin \& Birch 1966; Arnold \& Martin 2010). Adaptive trait introgression may be particularly important in a changing environment, since this mechanism can confer rapid evolutionary modification. In their pioneering experiments on the fruit fly Dacus tryoni, Lewontin \& Birch (1966) showed that the introduction of genes from another species (Dacus neohumeralis) was responsible for substantial changes in thermal optima, despite the absence of heterosis (i.e. $F_{1}$ hybrids and backcross generations were less fit than either progenitor). The introgressive transfer of thermally-adaptive gene combinations was thus identified as the mechanism underlying the rapid shift southward of this invasive Australian pest during the twentieth century (Lewontin \& Birch 1966).

Introgressive hybridization can lead to rapid evolution in symbiont lineages, with important effects on their mutualistic scope. Recent studies, particularly those focussed on plantpathogen interactions, have revealed extensive evolutionary developments arising from introgression. These can range from the acquisition of new host specificities and pathogenic aggressiveness to the adaptive divergence of new symbiont taxa (Brasier 1995, 2000; EtTouil et al. 1999; Brasier et al. 1999; Schardl \& Craven 2003). A particularly well studied 
case is the fungal agent of Dutch elm disease, Ophiostoma novo-ulmi, the cause of devastating losses of elms across Europe, Asia and America. While O. novo-ulmi $\times$ O. ulmi $\mathrm{F}_{1}$ hybrids are severely unfit and are quickly outcompeted by parental taxa, they serve to provide a bridge for unidirectional flow of genes, and possibly even viruses (Brasier 2001). O. novo-ulmi recently acquired the pathogenicity gene Pat 1 from $O$. ulmi via introgressive hybridization, a factor implicated in its increased virulence and concomitant intercontinental spread (Et-Touil et al. 1999).

Introgression can represent an especially potent macro-evolutionary force in haplontic organisms, particularly those with rapid generation times (Schardl \& Craven 2003). The potential for accelerated Symbiodinium evolution through introgressive hybridization may therefore have profound implications for corals in a shifting adaptive landscape. In particular, the transfer of genes between divergent Symbiodinium lineages has the potential to produce novel, host-compatible algal phenotypes, facilitating rapid adaptation of the symbiosis to warming ocean temperatures. In plants, introgressive hybridization can even lead to the transfer of chloroplasts between lineages ('chloroplast capture'; Rieseberg \& Soltis 1991). Chloroplast capture could have major implications for corals, since the symbiont chloroplast has been identified as a key target for oxidative damage, a precursor to thermal bleaching (reviewed in Lesser 2006). Yet the occurrence of introgression depends on many factors, including the frequency of hybridization events, the regions of the genome that are permeable to gene-flow, the relative fitness of each hybrid and backcross genotype, and the associated genotype $\times$ environment interactions (Barton 2001; Arnold 2007; Taylor et al. 2009). The abundance of putative Symbiodinium hybrids observed within many $P$. damicornis colonies suggests that a fitness advantage may be realized by some hybrid genotypes/classes under certain conditions; however further analyses of multiple diagnostic loci are needed to confirm this. The potential for introgression to expedite coral adaptation highlights the need to make timely progress in this area.

\subsubsection{Could hybridization be applied to improve reef resilience?}

The profound influence of hybridization on species evolution makes it a potentially powerful tool for restoring ecological systems. Attempts to improve habitats impacted by human activity can be enhanced by the directed translocation and/or outcrossing of individuals, with the intention of facilitating adaptation to changing conditions (assisted gene flow or AGF; 
Aitken \& Whitlock 2013). AGF is an emerging field that holds promise for mitigating maladaptation in a rapidly shifting adaptive landscape (Thomas et al. 2013; Aitken \& Whitlock 2013). However, there are several risks associated with restorative outcrossing, including the dilution of locally-adapted alleles (Montalvo \& Ellstrand 2000; Fenster \& Galloway 2000), the segregation of co-adapted gene complexes (Edmands 2007; Schiffers et al. 2013), the introduction of 'genetic pollution'(Rhymer \& Simberloff 1996; Hufford \& Mazer 2003), and in the case of symbiotic organisms, the stimulation of virulence (Brasier 2001). These factors could impart unforeseeable, possibly unmanageable consequences, and hence extensive feasibility assessments are necessary before any genetic interventions should be considered (Thomas et al. 2013).

For corals, the genetic enhancement of host-symbiont combinations through directed hybridization could enable the introduction of pre-adapted host-symbiont combinations to denuded reefs. These actions may expedite the restoration process and promote increased resilience to future environmental perturbation. Human-assisted evolution of symbiotic dinoflagellates could yield particularly effective results, due to their short generation times and known potential for rapid adaptation (van Oppen et al. 2011; Howells et al. 2011). Initially, establishing the efficacy of AGF in Symbiodinium would benefit from research aimed at inducing the sexual life cycle, determining the frequency and location of hybridization events, establishing barriers of genetic exchange, and identifying areas of the genome permeable to introgressive gene flow. While such 'evolutionary rescue' is fundamentally reactive in scope, this emerging area of research is a priority due to the continued reef degradation caused by human activity.

\subsubsection{Concluding remarks}

While usually deleterious in nature, hybridization can occasionally produce a fit individual whose genetic contribution can have a disproportionately large influence on adaptation. The resulting burst of genetic variation can provide evolutionary innovation two or three orders of magnitude greater than that offered by the gradual accumulation of mutations (Grant \& Grant 1994). The immense biodiversity and ecosystem services provided by the world's coral reefs emphasize the need to better understand the 'macro-evolutionary' potential of the many species constituting the coral holobiont. In particular, the integral roles of the symbiotic alga Symbiodinium in coral productivity, reef accretion and thermal bleaching highlight the critical 
importance of this research. This thesis takes a tentative step forward by providing indirect evidence of natural hybridization between two Symbiodinium lineages. Gaining a wider understanding of sexual reproduction and hybridization in both the host and the symbiont is needed to assess the potential for coral adaptation, both natural and human-assisted, as our destructive activity continues to jeopardize their future. 


\section{References}

Abbott R, Albach D, Ansell S et al. (2013) Hybridization and speciation. Journal of Evolutionary Biology, 26, 229-246.

Abrego D, Ulstrup KE, Willis BL, van Oppen MJH (2008) Species-specific interactions between algal endosymbionts and coral hosts define their bleaching response to heat and light stress. Proceedings of the Royal Society B, 275, 2273-2282.

Aitken SN, Whitlock MC (2013) Assisted gene flow to facilitate local adaptation to climate change. Annual Review of Ecology, Evolution, and Systematics, 44, 367-388.

Allemand D, Tambutté É, Zoccola D, Tambutté S (2011) Coral calcification, cells to reefs. In: Coral Reefs: An Ecosystem in Transition (eds Dubinsky Z, Stambler N), pp. 119150. Springer, New York.

Allen GR, Paxton JR (1974) A tropical outpost in the Pacific. Australian Natural History, 18, $50-55$.

Altschul SF, Gish W, Miller W, Myers EW, Lipman DJ (1990) Basic local alignment search tool. Journal of Molecular Biology, 215, 403-410.

Álvarez I, Wendel JF (2003) Ribosomal ITS sequences and plant phylogenetic inference. Molecular Phylogenetics and Evolution, 29, 417-434.

Anderson E (1949) Introgressive Hybridization. John Wiley and Sons, New York.

Anderson DM, Kulis DM, Binder BJ (1984) Sexuality and cyst formation in the dinoflagellate Gonyaulax tamarensis: cyst yield in batch cultures. Journal of Phycology, 20, 418-425.

Anderson E, Stebbins GL Jr (1954) Hybridization as an evolutionary stimulus. Evolution, 8, 378-388.

Andersson L (1990) The driving force: species concepts and ecology. Taxon, 39, 375-382.

Andras JP, Kirk NL, Harvell CD (2011) Range-wide population genetic structure of Symbiodinium associated with the Caribbean Sea fan coral, Gorgonia ventalina. Molecular Ecology, 20, 2525-2542.

Anthony KRN, Kline DI, Diaz-Pulido G, Dove S, Hoegh-Guldberg O (2008) Ocean acidification causes bleaching and productivity loss in coral reef builders. Proceedings of the National Academy of Sciences of the United States of America, 105, 1744217446.

Arif C, Daniels C, Bayer T et al. (2014) Assessing Symbiodinium diversity in scleractinian corals via next-generation sequencing- based genotyping of the ITS2 rDNA region. Molecular Ecology, 23, 4418-4433. 
Arnheim N, Krystal M, Schmickel R et al. (1980) Molecular evidence for genetic exchanges among ribosomal genes on nonhomologous chromosomes in man and apes. Proceedings of the National Academy of Sciences of the United States of America, 77, 7323-7327.

Arnold SJ (1983) Morphology, performance and fitness. American Zoologist, 23, 347-361.

Arnold ML (1997) Natural Hybridization and Evolution. Oxford University Press, Oxford.

Arnold ML (2007) Evolution through Genetic Exchange. Oxford University Press, Oxford.

Arnold ML, Hodges SA (1995) Are natural hybrids fit or unfit relative to their parents? Trends in Ecology and Evolution, 10, 67-71.

Arnold ML, Martin NH (2010) Hybrid fitness across time and habitats. Trends in Ecology and Evolution, 25, 530-536.

Van Baalen M, Sabelis MW (1995) The dynamics of multiple infection and the evolution of virulence. The American Naturalist, 146, 881-910.

Bachvaroff TR, Place AR (2008) From stop to start: tandem gene arrangement, copy number and trans-splicing sites in the dinoflagellate Amphidinium carterae. PLoS ONE, $\mathbf{3}$, e2929.

Baillie BK, Monje V, Silvestre V, Sison M, Belda-Baillie CA (1998) Allozyme electrophoresis as a tool for distinguishing different zooxanthellae symbiotic with giant clams. Proceedings of the Royal Society of London B, 265, 1949-1956.

Baillie BK, Belda-Baillie CA, Silvestre V et al. (2000) Genetic variation in Symbiodinium isolates from giant clams based on random-amplified-polymorphic DNA (RAPD) patterns. Marine Biology, 136, 829-836.

Baird AH, Bhagooli R, Ralph PJ, Takahashi S (2008) Coral bleaching: the role of the host. Trends in Ecology and Evolution, 24, 16-20.

Baird AH, Guest JR, Willis BL (2009) Systematic and biogeographical patterns in the reproductive biology of scleractinian corals. Annual Review of Ecology, Evolution, and Systematics, 40, 551-571.

Baker AC (2001) Reef corals bleach to survive change. Nature, 411, 765-766.

Baker AC (2003) Flexibility and specificity in coral-algal symbiosis: diversity, ecology, and biogeography of Symbiodinium. Annual Review of Ecology, Evolution, and Systematics, 34, 661-689.

Baker AC, Rowan R (1997) Diversity of symbiotic dinoflagellates (zooxanthellae) in scleractinian corals of the Caribbean and Eastern Pacific. Proceedings of the 8th International Coral Reef Symposium, 2, 1301-1306.

Baker AC, Starger CJ, McClanahan TR, Glynn PW (2004) Corals' adaptive response to climate change. Nature, $\mathbf{4 3 0}, 741$. 
Baker AC, Romanski AM (2007) Multiple symbiotic partnerships are common in scleractinian corals, but not in octocorals: Comment on Goulet (2006). Marine Ecology Progress Series, 335, 237-242.

Baldwin BG, Sanderson MJ, Porter JM et al. (1995) The ITS region of nuclear ribosomal DNA: a valuable source of evidence on angiosperm phylogeny. Annals of the Missouri Botanical Garden, 82, 247-277.

Banaszak AT, Iglestas-Prieto R, Trench RK (1993) Scrippsiella velellae sp. nov. (Peridiniales) and Gloeodinium viscum sp. nov. (Phytodiniales), dinoflagellate symbionts of two hydrozoans (Cnidaria). Journal of Phycology, 29, 517-528.

Banaszak AT, LaJeunesse TC, Trench RK (2000) The synthesis of mycosporine-like amino acids (MAAs) by cultured, symbiotic dinoflagellates. Journal of Experimental Marine Biology and Ecology, 249, 219-233.

Barbrook AC, Voolstra CR, Howe CJ (2014) The chloroplast genome of a Symbiodinium sp. clade C3 isolate. Protist, 165, 1-13.

Barnes DJ, Chalker BE (1990) Calcification and photosynthesis in reef-building corals and algae. In: Ecosystems of the World, 25: Coral Reefs (ed Dubinsky Z), pp. 109-131. Elsevier, New York.

Barrett LG, Thrall PH, Burdon JJ (2007) Evolutionary diversification through hybridization in a wild host-pathogen interaction. Evolution, 61, 1613-1621.

Barton NH (2001) The role of hybridization in evolution. Molecular Ecology, 10, 551-568.

Barton N, Bengtsson BO (1986) The barrier to genetic exchange between hybridising populations. Heredity, 57, 357-376.

Baums IB, Devlin-Durante MK, LaJeunesse TC (2014) New insights into the dynamics between reef corals and their associated dinoflagellate endosymbionts from population genetic studies. Molecular Ecology, DOI: 10.1111/mec.12788.

Bell JJ, Davy SK, Jones T, Taylor MW, Webster NS (2013) Could some coral reefs become sponge reefs as our climate changes? Global Change Biology, 19, 2613-2624.

Bell MA, Travis MP (2005) Hybridization, transgressive segregation, genetic covariation, and adaptive radiation. Trends in Ecology and Evolution, 20, 358-361.

Berkelmans R, van Oppen MJH (2006) The role of zooxanthellae in the thermal tolerance of corals: a "nugget of hope" for coral reefs in an era of climate change. Proceedings of the Royal Society B, 273, 2305-2312.

Bhagooli R, Hidaka M (2003) Comparison of stress susceptibility of in hospite and isolated zooxanthellae among five coral species. Journal of Experimental Marine Biology and Ecology, 291, 181-197. 
Bhagooli R, Hidaka M (2004) Release of zooxanthellae with intact photosynthetic activity by the coral Galaxea fascicularis in response to high temperature stress. Marine Biology, 145, 329-337.

Blackburn SI, Bolch CJS, Haskard KA, Hallegraeff GM (2001) Reproductive compatibility among four global populations of the toxic dinoflagellate Gymnodinium catenatum (Dinophyceae). Phycologia, 40, 78-87.

Blank RJ, Huss VAR (1989) DNA divergency and speciation in Symbiodinium (Dinophyceae). Plant Systematics and Evolution, 163, 153-163.

Brasier CM (1995) Episodic selection as a force in fungal microevolution, with special reference to clonal speciation and hybrid introgression. Canadian Journal of Botany, 73, S1213-S1221.

Brasier C (2000) The rise of the hybrid fungi. Nature, 405, 134-135.

Brasier CM (2001) Rapid evolution of introduced plant pathogens via interspecific hybridization. BioScience, 51, 123-133.

Brasier CM, Cooke DEL, Duncan JM (1999) Origin of a new Phytophthora pathogen through interspecific hybridization. Proceedings of the National Academy of Sciences of the United States of America, 96, 5878-5883.

Briggs JC (1999) Coincident biogeographic patterns: Indo-West Pacific Ocean. Evolution, $\mathbf{5 3}, 326-335$.

Brodie J, Fabricius K, De'ath G, Okaji K (2005) Are increased nutrient inputs responsible for more outbreaks of crown-of-thorns starfish? An appraisal of the evidence. Marine Pollution Bulletin, 51, 266-278.

Brosnahan ML, Kulis DM, Solow AR et al. (2010) Outbreeding lethality between toxic Group I and nontoxic Group III Alexandrium tamarense spp. isolates: Predominance of heterotypic encystment and implications for mating interactions and biogeography. Deep Sea Research Part II: Topical Studies in Oceanography, 57, 175-189.

Brown DD, Wensink PC, Jordan E (1972) A comparison of the ribosomal DNA's of Xenopus laevis and Xenopus mulleri: the evolution of tandem genes. Journal of Molecular Biology, 63, 57-64.

Bruno JF, Selig ER, Casey KS et al. (2007) Thermal stress and coral cover as drivers of coral disease outbreaks. PLoS Biology, 5, e124.

Bryant D, Burke L, McManus J, Spalding M (1998) Reefs at Risk: A Map-based Indicator of Threats to the World's Coral Reefs. World Resources Institute, Washington D.C.

Buckler ES IV, Ippolito A, Holtsford TP (1997) The evolution of ribosomal DNA: divergent paralogues and phylogenetic implications. Genetics, 145, 821-832. 
Buddemeier RW, Fautin DG (1993) Coral bleaching as an adaptive mechanism. BioScience, 43, 320-326.

Buerkle CA, Morris RJ, Asmussen MA, Rieseberg LH (2000) The likelihood of homoploid hybrid speciation. Heredity, 84, 441-451.

Burke L, Reytar K, Spalding M, Perry A (2011) Reefs at Risk Revisited. World Resources Institute, Washington D.C.

Burnham KP, Anderson DR (2002) Model Selection and Multimodel Inference: A Practical Information-Theoretic Approach (2nd edition). Springer, New York.

Burriesci MS, Raab TK, Pringle JR (2012) Evidence that glucose is the major transferred metabolite in dinoflagellate-cnidarian symbiosis. The Journal of Experimental Biology, 215, 3467-3477.

Byler KA, Carmi-Veal M, Fine M, Goulet TL (2013) Multiple symbiont acquisition strategies as an adaptive mechanism in the coral Stylophora pistillata. PLoS ONE, $\mathbf{8}$, e59596.

Cadenas E (1989) Biochemistry of oxygen toxicity. Annual Review of Biochemistry, 58, 79110.

Cantin NE, van Oppen MJH, Willis BL, Mieog JC, Negri AP (2009) Juvenile corals can acquire more carbon from high-performance algal symbionts. Coral Reefs, 28, 405-414.

Carlos AA, Baillie BK, Kawachi M, Maruyama T (1999) Phylogenetic position of Symbiodinium (Dinophyceae) isolates from Tridacnids (Bivalvia), Cardiids (Bivalvia), a sponge (Porifera), a soft coral (Anthozoa), and a free-living strain. Journal of Phycology, 35, 1054-1062.

Carpenter KE, Abrar M, Aeby G et al. (2008) One-third of reef-building corals face elevated extinction risk from climate change and local impacts. Science, 321, 560-563.

Carté BK (1996) Biomedical potential of marine natural products. BioScience, 46, 271-286.

Casteleyn G, Adams NG, Vanormelingen P et al. (2009) Natural hybrids in the marine diatom Pseudo-nitzschia pungens (Bacillariophyceae): genetic and morphological evidence. Protist, 160, 343-354.

De Castro O, Di Maio A, Lozada García JA et al. (2013) Plastid DNA sequencing and nuclear SNP genotyping help resolve the puzzle of central American Platanus. Annals of Botany, 112, 589-602.

Cesar H (1996) Economic Analysis of Indonesian Coral Reefs. The World Bank, Washington D.C.

Chalker BE, Dunlap WC, Oliver JK (1983) Bathymetric adaptations of reef-building corals at Davies Reef, Great Barrier Reef, Australia. II. Light saturation curves for photosynthesis and respiration. Journal of Experimental Marine Biology and Ecology, 73, 37-56. 
Chang SS, Prézelin BB, Trench RK (1983) Mechanisms of photoadaptation in three strains of the symbiotic dinoflagellate Symbiodinium microadriaticum. Marine Biology, 76, 219229.

Charlesworth D, Charlesworth B (1987) Inbreeding depression and its evolutionary consequences. Annual Review of Ecology and Systematics, 18, 237-268.

Chen CA, Wang J-T, Fang L-S, Yang Y-W (2005) Fluctuating algal symbiont communities in Acropora palifera (Scleractinia: Acroporidae) from Taiwan. Marine Ecology Progress Series, 295, 113-121.

Chesnick JM, Cox ER (1987) Synchronized sexuality of an algal symbiont and its dinoflagellate host, Peridinium balticum (Levander) Lemmermann. Biosystems, 21, 6978 .

Chi J, Parrow MW, Dunthorn M (2014) Cryptic sex in Symbiodinium (Alveolata, Dinoflagellata) is supported by an inventory of meiotic genes. Journal of Eukaryotic Microbiology, 61, 322-327.

Clay K, Schardl C (2002) Evolutionary origins and ecological consequences of endophyte symbiosis with grasses. The American Naturalist, 160, S99-S127.

Clement M, Posada D, Crandall KA (2000) TCS: a computer program to estimate gene genealogies. Molecular Ecology, 9, 1657-1659.

Coates AG, Jackson JBC (1987) Clonal growth, algal symbiosis, and reef formation by corals. Paleobiology, 13, 363-378.

Coffroth MA, Santos SR (2005) Genetic diversity of symbiotic dinoflagellates in the genus Symbiodinium. Protist, 156, 19-34.

Coffroth MA, Santos SR, Goulet TL (2001) Early ontogenetic expression of specificity in a cnidarian-algal symbiosis. Marine Ecology Progress Series, 222, 85-96.

Coffroth MA, Lewis CF, Santos SR, Weaver JL (2006) Environmental populations of symbiotic dinoflagellates in the genus Symbiodinium can initiate symbioses with reef cnidarians. Current Biology, 16, R985-R987.

Coffroth MA, Poland DM, Petrou EL, Brazeau DA, Holmberg JC (2010) Environmental symbiont acquisition may not be the solution to warming seas for reef-building corals. PLOS ONE, 5, e13258.

Coleman AW, Suarez A, Goff LJ (1994) Molecular delineation of species and syngens in Volvocacean green algae (Chlorophyta). Journal of Phycology, 30, 80-90.

Coles SL, Jokiel PL (1978) Synergistic effects of temperature, salinity and light on the hermatypic coral Montipora verrucosa. Marine Biology, 195, 187-195. 
Colombo-Pallotta MF, Rodríguez-Román A, Iglesias-Prieto R (2010) Calcification in bleached and unbleached Montastraea faveolata: evaluating the role of oxygen and glycerol. Coral Reefs, 29, 899-907.

Combosch DJ, Vollmer S V (2013) Mixed asexual and sexual reproduction in the IndoPacific reef coral Pocillopora damicornis. Ecology and Evolution, 3, 3379-3387.

Combosch DJ, Guzman HM, Schuhmacher H, Vollmer S V (2008) Interspecific hybridization and restricted trans-Pacific gene flow in the Tropical Eastern Pacific Pocillopora. Molecular Ecology, 17, 1304-1312.

Cook CB (1971) Transfer of 35'-labeled material from food ingested by Aiptasia sp. to its endosymbiotic zooxanthellae. In: Experimental Coelenterate Biology (eds Lenhoff HM, Muscatine L, Davis LV), pp. 218-224. University of Hawaii Press, Honolulu.

Correa AMS, Baker AC (2009) Understanding diversity in coral-algal symbiosis: a clusterbased approach to interpreting fine-scale genetic variation in the genus Symbiodinium. Coral Reefs, 28, 81-93.

Correa AMS, Baker AC (2011) Disaster taxa in microbially mediated metazoans: how endosymbionts and environmental catastrophes influence the adaptive capacity of reef corals. Global Change Biology, 17, 68-75.

Correa AMS, McDonald MD, Baker AC (2009) Development of clade-specific Symbiodinium primers for quantitative PCR (qPCR) and their application to detecting clade D symbionts in Caribbean corals. Marine Biology, 156, 2403-2411.

Coskun S, Alsmadi O (2007) Whole genome amplification from a single cell: a new era for preimplantation genetic diagnosis. Prenatal Diagnosis, 27, 297-302.

Costanza R, D'Arge R, de Groot R et al. (1997) The value of the world's ecosystem services and natural capital. Nature, 387, 253-260.

Cronn RC, Zhao X, Paterson AH, Wendel JF (1996) Polymorphism and concerted evolution in a tandemly repeated gene family: 5S ribosomal DNA in diploid and allopolyploid cottons. Journal of Molecular Evolution, 42, 685-705.

Crossland CJ, Barnes DJ (1977) Gas-exchange studies with the staghorn coral Acropora acuminata and its zooxanthellae. Marine Biology, 40, 185-194.

Crow JF (1994) Advantages of sexual reproduction. Developmental Genetics, 15, 205-213.

Cunning R, Baker AC (2013) Excess algal symbionts increase the susceptibility of reef corals to bleaching. Nature Climate Change, 3, 259-262.

Cunning R, Glynn PW, Baker AC (2013) Flexible associations between Pocillopora corals and Symbiodinium limit utility of symbiosis ecology in defining species. Coral Reefs, 32, 795-801. 
Davies PS (1984) The role of zooxanthellae in the nutritional energy requirements of Pocillopora eydouxi. Coral Reefs, 2, 181-186.

Davy SK, Turner JR (2003) Early development and acquisition of zooxanthellae in the temperate symbiotic sea anemone Anthopleura ballii (Cocks). The Biological Bulletin, 205, 66-72.

Davy SK, Lucas IAN, Turner JR (1997) Uptake and persistence of homologous and heterologous zooxanthellae in the temperate sea anemone Cereus pedunculatus (Pennant). The Biological Bulletin, 192, 208-216.

Davy SK, Allemand D, Weis VM (2012) Cell biology of cnidarian-dinoflagellate symbiosis. Microbiology and Molecular Biology Reviews, 76, 229-261.

Dean FB, Hosono S, Fang L et al. (2002) Comprehensive human genome amplification using multiple displacement amplification. Proceedings of the National Academy of Sciences of the United States of America, 99, 5261-5266.

Demuth JP, Wade MJ (2005) On the theoretical and empirical framework for studying genetic interactions within and among species. The American Naturalist, 165, 524-536.

Destombe C, Cembella A (1990) Mating-type determination, gametic recognition and reproductive success in Alexandrium excavatum (Gonyaulacales, Dinophyta), a toxic red-tide dinoflagellate. Phycologia, 29, 316-325.

Diekmann OE, Bak RPM, Tonk L, Stam WT, Olsen JL (2002) No habitat correlation of zooxanthellae in the coral genus Madracis on a Curaçao reef. Marine Ecology Progress Series, 227, 221-232.

Dobzhansky T (1968) On some fundamental concepts of Darwinian biology. In: Evolutionary Biology (eds Dobzhansky T, Hecht MK, Steere WC), pp. 1-34. Plenum Press, New York.

Douglas AE (1998) Host benefit and the evolution of specialization in symbiosis. Heredity, 81, 599-603.

Dover G (1982) Molecular drive: a cohesive mode of species evolution. Nature, 299, 111117.

Drew EA (1972) The biology and physiology of alga-invertebrate symbioses. II. The density of symbiotic algal cells in a number of hermatypic hard corals and alcyonarians from various depths. Journal of Experimental Marine Biology and Ecology, 9, 71-75.

Dunn SR, Weis VM (2009) Apoptosis as a post-phagocytic winnowing mechanism in a coraldinoflagellate mutualism. Environmental Microbiology, 11, 268-276.

Edmands S (2007) Between a rock and a hard place: evaluating the relative risks of inbreeding and outbreeding for conservation and management. Molecular Ecology, 16, $463-475$. 
Edvardsen B, Shalchian-Tabrizi K, Jakobsen KS et al. (2003) Genetic variability and molecular phylogeny of Dinophysis species (Dinophyceae) from Norwegian waters inferred from single cell analyses of rDNA. Journal of Phycology, 39, 395-408.

Elbrächter M (2003) Dinophyte reproduction: progress and conflicts. Journal of Phycology, 39, 629-632.

Epifanio JM, Phillipp DP (1997) Sources for misclassifying genealogical origins in mixed hybrid populations. Journal of Heredity, 88, 62-65.

Et-Touil A, Brasier CM, Bernier L (1999) Localization of a pathogenicity gene in Ophiostoma novo-ulmi and evidence that it may be introgressed from $O$. ulmi. Molecular Plant-Microbe Interactions, 12, 6-15.

Fabricius KE, Langdon C, Uthicke S et al. (2011) Losers and winners in coral reefs acclimatized to elevated carbon dioxide concentrations. Nature Climate Change, 1, 165169.

Fadlallah YH (1983) Sexual reproduction, development and larval biology in scleractinian corals. Coral Reefs, 2, 129-150.

Falkowski PG, Dubinsky Z (1981) Light-shade adaptation of Stylophora pistillata, a hermatypic coral from the Gulf of Eilat. Nature, 289, 172-174.

Fautin DG, Buddemeier RW (2004) Adaptive bleaching: a general phenomenon. Hydrobiologia, 530/531, 459-467.

Felsenstein J (1974) The evolutionary advantage of recombination. Genetics, 78, 737-756.

Fenster CB, Galloway LF (2000) Inbreeding and outbreeding depression in natural populations of Chamaecrista fasciculata (Fabaceae). Conservation Biology, 14, 14061412.

Ferrier-Pagès C, Gattuso J-P, Dallot S, Jaubert J (2000) Effect of nutrient enrichment on growth and photosynthesis of the zooxanthellate coral Stylophora pistillata. Coral Reefs, 19, 103-113.

Figueroa RI, Garcés E, Bravo I (2007) Comparative study of the life cycles of Alexandrium tamutum and Alexandrium minutum (Gonyaulacales, Dinophyceae) in culture. Journal of Phycology, 43, 1039-1053.

Figueroa RI, Vázquez JA, Massanet A, Murado MA, Bravo I (2011) Interactive effects of salinity and temperature on planozygote and cyst formation of Alexandrium minutum (Dinophyceae) in culture. Journal of Phycology, 47, 13-24.

Figueroa RI, Cuadrado A, Stüken A, Rodríguez F, Fraga S (2014) Ribosomal DNA organization patterns within the dinoflagellate genus Alexandrium as revealed by FISH: life cycle and evolutionary implications. Protist, 165, 343-363. 
Finney JC, Pettay DT, Sampayo EM et al. (2010) The relative significance of host-habitat, depth, and geography on the ecology, endemism, and speciation of coral endosymbionts in the genus Symbiodinium. Microbial Ecology, 60, 250-263.

Fisher PL, Malme MK, Dove S (2011) The effect of temperature stress on coralSymbiodinium associations containing distinct symbiont types. Coral Reefs, 31, 473485.

Fitt WK (1984) The role of chemosensory behavior of Symbiodinium microadriaticum, intermediate hosts, and host behavior in the infection of coelenterates and molluscs with zooxanthellae. Marine Biology, 81, 9-17.

Fitt WK, Trench RK (1983) The relation of diel patterns of cell division to diel patterns of motility in the symbiotic dinoflagellate Symbiodinium microadriaticum Freudenthal in culture. New Phytologist, 94, 421-432.

Fitt WK, Warner ME (1995) Bleaching patterns of four species of Caribbean reef corals. The Biological Bulletin, 189, 298-307.

Fitt WK, Mcfarland FK, Warner ME, Chilcoa GC (2000) Seasonal patterns of tissue biomass and densities of symbiotic dinoflagellates in reef corals and relation to coral bleaching. Limnology and Oceanography, 45, 677-685.

Fitt WK, Gates RD, Hoegh-Guldberg O et al. (2009) Response of two species of Indo-Pacific corals, Porites cylindrica and Stylophora pistillata, to short-term thermal stress: The host does matter in determining the tolerance of corals to bleaching. Journal of Experimental Marine Biology and Ecology, 373, 102-110.

Fitzpatrick BM (2012) Estimating ancestry and heterozygosity of hybrids using molecular markers. BMC Evolutionary Biology, 12, 131.

Flores-Moya A, Costas E, López-Rodas V (2008) Roles of adaptation, chance and history in the evolution of the dinoflagellate Prorocentrum triestinum. Naturwissenschaften, $\mathbf{9 5}$, 697-703.

Flot J-F, Hespeels B, Li X et al. (2013) Genomic evidence for ameiotic evolution in the bdelloid rotifer Adineta vaga. Nature, 500, 453-457.

Frade PR, Englebert N, Faria J, Visser PM, Bak RPM (2008) Distribution and photobiology of Symbiodinium types in different light environments for three colour morphs of the coral Madracis pharensis: is there more to it than total irradiance? Coral Reefs, 27, 913925.

Frank SA (1996a) Host control of symbiont transmission: the separation of symbionts into germ and soma. The American Naturalist, 148, 1113-1124.

Frank SA (1996b) Host-symbiont conflict over the mixing of symbiotic lineages. Proceedings of the Royal Society of London B, 263, 339-344. 
Franklin EC, Stat M, Pochon X, Putnam HM, Gates RD (2012) GeoSymbio: a hybrid, cloudbased web application of global geospatial bioinformatics and ecoinformatics for Symbiodinium-host symbioses. Molecular Ecology Resources, 12, 369-373.

Freudenthal HD (1962) Symbiodinium gen. nov. and Symbiodinium microadriaticum sp. nov., a zooxanthella: taxonomy, life cycle, and morphology. Journal of Eukaryotic Microbiology, 9, 45-52.

Ganley ARD, Scott B (2002) Concerted evolution in the ribosomal RNA genes of an Epichlö̈ endophyte hybrid: comparison between tandemly arranged rDNA and dispersed 5S rrn genes. Fungal Genetics and Biology, 35, 39-51.

Gardner JPA (1997) Hybridization in the sea. Advances in Marine Biology, 31, 1-78.

Garrison VH, Ward G (2012) Transplantation of storm-generated coral fragments to enhance Caribbean coral reefs: a successful method but not a solution. Revista de Biología Tropical, 60, 59-70.

Gates RD, Baghdasarian G, Muscatine L (1992) Temperature stress causes host cell detachment in symbiotic cnidarians: implications for coral bleaching. The Biological Bulletin, 182, 324-332.

Gates RD, Muscatine L (1992) Three methods for isolating viable anthozoan endoderm cells with their intracellular symbiotic dinoflagellates. Coral Reefs, 11, 143-145.

Gates RD, Hoegh-Guldberg O, McFall-Ngai MJ, Bil KY, Muscatine L (1995) Free amino acids exhibit anthozoan "host factor" activity: They induce the release of photosynthate from symbiotic dinoflagellates in vitro. Proceedings of the National Academy of Sciences of the United States of America, 92, 7430-7434.

Geneious version 7.0 created by Biomatters. Available from http://www.geneious.com

Gleason DF, Wellington GM (1993) Ultraviolet radiation and coral bleaching. Nature, 365, 836-838.

Glynn PW (1996) Coral reef bleaching: facts, hypotheses and implications. Global Change Biology, 2, 495-509.

Gompert Z, Fordyce JA, Forister ML, Shapiro AM, Nice CC (2006) Homoploid hybrid speciation in an extreme habitat. Science, 314, 1923-1925.

Gordo I, Charlesworth B (2000) The degeneration of asexual haploid populations and the speed of Muller's ratchet. Genetics, 154, 1379-1387.

Goreau TF, Goreau NI (1959) The physiology of skeleton formation in corals. II. Calcium deposition by hermatypic corals under various conditions in the reef. The Biological Bulletin, 117, 239-250.

Goulet TL (2006) Most corals may not change their symbionts. Marine Ecology Progress Series, 321, 1-7. 
Goulet TL, Coffroth MA (2003a) Stability of an octocoral-algal symbiosis over time and space. Marine Ecology Progress Series, 250, 117-124.

Goulet TL, Coffroth MA (2003b) Genetic composition of zooxanthellae between and within colonies of the octocoral Plexaura kuna, based on small subunit rDNA and multilocus DNA fingerprinting. Marine Biology, 142, 233-239.

Goulet TL, Cook CB, Goulet D (2005) Effect of short-term exposure to elevated temperatures and light levels on photosynthesis of different host-symbiont combinations in the Aiptasia pallida/Symbiodinium symbiosis. Limnology and Oceanography, 50, 1490-1498.

Grant V (1981) Plant Speciation. Columbia University Press, New York.

Grant PR, Grant BR (1994) Phenotypic and genetic effects of hybridization in Darwin' s finches. Evolution, 48, 297-316.

Grant PR, Grant BR, Petren K (2005) Hybridization in the recent past. The American Naturalist, 166, 56-67.

Green DM (2005) Designatable units for species status assessment and protection. Conservation Biology, 19, 1813-1820.

Green EA, Davies SW, Matz M V., Medina M (2014) Quantifying cryptic Symbiodinium diversity within Orbicella faveolata and Orbicella franksi at the Flower Garden Banks, Gulf of Mexico. PeerJ, 2, e386.

Green AL, Mous PJ (2008) Delineating the Coral Triangle, its Ecoregions and Functional Seascapes. Version 5.0. TNC Coral Triangle Program Report 1/08. 44 pp.

Grottoli AG, Rodrigues LJ, Palardy JE (2006) Heterotrophic plasticity and resilience in bleached corals. Nature, 440, 1186-1189.

Grube M, White JF, Seckbach J (2010) Symbioses and stress. In: Symbioses and Stress: Cellular Origin, Life in Extreme Habitats and Astrobiology, Volume 17 (eds Seckbach J, Grube M), pp. 21-36. Springer, Dordrecht.

Hackett JD, Anderson DM, Erdner DL, Bhattacharya D (2004) Dinoflagellates: a remarkable evolutionary experiment. American Journal of Botany, 91, 1523-1534.

Hamilton CE, Faeth SH, Dowling TE (2009) Distribution of hybrid fungal symbionts and environmental stress. Microbial Ecology, 58, 408-413.

Handyside AH, Robinson MD, Simpson RJ et al. (2004) Isothermal whole genome amplification from single and small numbers of cells: a new era for preimplantation genetic diagnosis of inherited disease. Molecular Human Reproduction, 10, 767-772.

Hansen G, Daugbjerg N (2009) Symbiodinium natans sp. nov.: a "free-living" dinoflagellate from Tenerife (Northeast-Atlantic Ocean). Journal of Phycology, 45, 251-263. 
Harriott VJ, Banks SA (2002) Latitudinal variation in coral communities in eastern Australia: a qualitative biophysical model of factors regulating coral reefs. Coral Reefs, 21, 83-94.

Harriott VJ, Harrison PL, Banks SA (1995) The coral communities of Lord Howe Island. Marine and Freshwater Research, 46, 457-465.

Harrison PL, Dalton SJ, Carroll AG (2011) Extensive coral bleaching on the world's southernmost coral reef at Lord Howe Island, Australia. Coral Reefs, 30, 775.

Harrison PL, Wallace CC (1990) Reproduction, dispersal and recruitment of scleractinian corals. In: Ecosystems of the World, 25. Coral Reefs (ed Dubinsky Z), pp. 133-207. Elsevier, Amsterdam.

Hart MC, Green DH, Bresnan E, Bolch CJ (2007) Large subunit ribosomal RNA gene variation and sequence heterogeneity of Dinophysis (Dinophyceae) species from Scottish coastal waters. Harmful Algae, 6, 271-287.

Hasegawa M, Kishino H, Yano T (1985) Dating of the human-ape splitting by a molecular clock of mitochondrial DNA. Journal of Molecular Evolution, 22, 160-174.

Hawkins TD, Krueger T, Becker S, Fisher PL, Davy SK (2014) Differential nitric oxide synthesis and host apoptotic events correlate with bleaching susceptibility in reef corals. Coral Reefs, 33, 141-153.

Hill R, Ralph PJ (2007) Post-bleaching viability of expelled zooxanthellae from the scleractinian coral Pocillopora damicornis. Marine Ecology Progress Series, 352, 137144.

Hoegh-Guldberg O (1999) Climate change, coral bleaching and the future of the world's coral reefs. Marine and Freshwater Research, 50, 839-866.

Hoegh-Guldberg O, Jones RJ, Ward S, Loh WK (2002) Is coral bleaching really adaptive? Nature, 415, 601-602.

Hoegh-Guldberg O, Mumby PJ, Hooten AJ et al. (2007) Coral reefs under rapid climate change and ocean acidification. Science, 318, 1737-1742.

Hoffmann AA, Sgrò CM (2011) Climate change and evolutionary adaptation. Nature, 470, 479-485.

Hollingsworth LL, Kinzie RA III, Lewis TD, Krupp DA, Leong J-AC (2005) Phototaxis of motile zooxanthellae to green light may facilitate symbiont capture by coral larvae. Coral Reefs, 24, 523.

Hou Y, Lin S (2009) Distinct gene number-genome size relationships for eukaryotes and noneukaryotes: gene content estimation for dinoflagellate genomes. PLoS ONE, 4, e6978.

Howells EJ, van Oppen MJH, Willis BL (2009) High genetic differentiation and cross-shelf patterns of genetic diversity among Great Barrier Reef populations of Symbiodinium. Coral Reefs, 28, 215-225. 
Howells EJ, Beltran VH, Larsen NW et al. (2011) Coral thermal tolerance shaped by local adaptation of photosymbionts. Nature Climate Change, 2, 116-120.

Howells EJ, Willis BL, Bay LK, van Oppen MJH (2013) Spatial and temporal genetic structure of Symbiodinium populations within a common reef-building coral on the Great Barrier Reef. Molecular Ecology, 22, 3693-3708.

Hufford KM, Mazer SJ (2003) Plant ecotypes: genetic differentiation in the age of ecological restoration. Trends in Ecology and Evolution, 18, 147-155.

Hughes TP, Baird AH, Bellwood DR et al. (2003) Climate change, human impacts, and the resilience of coral reefs. Science, 301, 929-933.

Humphreys MW, Thomas HM, Morgan WG et al. (1995) Discriminating the ancestral progenitors of hexaploid Festuca arundinacea using genomic in situ hybridization. Heredity, 75, 171-174.

Iglesias-Prieto R, Trench RK (1994) Acclimation and adaptation to irradiance in symbiotic dinoflagellates. I. Responses of the photosynthetic unit to changes in photon flux density. Marine Ecology Progress Series, 113, 163-175.

Iglesias-Prieto R, Trench RK (1997) Acclimation and adaptation to irradiance in symbiotic dinoflagellates. II. Response of chlorophyll-protein complexes to different photon-flux densities. Marine Biology, 130, 23-33.

Iglesias-Prieto R, Matta JL, Robins WA, Trench RK (1992) Photosynthetic response to elevated temperature in the symbiotic dinoflagellate Symbiodinium microadriaticum in culture. Proceedings of the National Academy of Sciences of the United States of America, 89, 10302-10305.

Iglesias-Prieto R, Beltrán VH, LaJeunesse TC, Reyes-Bonilla H, Thomé PE (2004) Different algal symbionts explain the vertical distribution of dominant reef corals in the eastern Pacific. Proceedings of the Royal Society of London B, 271, 1757-1763.

Ingvarsson PK, Whitlock MC (2000) Heterosis increases the effective migration rate. Proceedings of the Royal Society of London B, 267, 1321-1326.

Jeffrey SW, Humphrey GF (1975) New spectrophotometric equations for determining chlorophylls $a, b, c_{1}$ and $c_{2}$ in higher plants, algae and natural phytoplankton. Biochemie und Physiologie der Pflanzen, 167, 191-194.

Jeong HJ, Lee SY, Kang NS et al. (2014) Genetics and morphology characterize the dinoflagellate Symbiodinium voratum, n. sp., (Dinophyceae) as the sole representative of Symbiodinium Clade E. Journal of Eukaryotic Microbiology, 61, 75-94.

Johnson JB, Omland KS (2004) Model selection in ecology and evolution. Trends in Ecology and Evolution, 19, 101-108.

Jokiel PL, Coles SL (1977) Effects of temperature on the mortality and growth of Hawaiian reef corals. Marine Biology, 43, 201-208. 
Jokiel PL, Maracios JE, Franzisket L (1978) Coral growth: buoyant weight technique. In: Coral Reefs: Research Methods (eds Stoddard DR, Juhannes RE), pp. 529-541. UNESCO, Paris.

Jones A, Berkelmans R (2010) Potential costs of acclimatization to a warmer climate: growth of a reef coral with heat tolerant vs. sensitive symbiont types. PLOS ONE, 5, e10437.

Jones AM, Berkelmans R, van Oppen MJH, Mieog JC, Sinclair W (2008) A community change in the algal endosymbionts of a scleractinian coral following a natural bleaching event: field evidence of acclimatization. Proceedings of the Royal Society B, 275, 13591365.

Jones RJ, Yellowlees D (1997) Regulation and control of intracellular algae (= zooxanthellae) in hard corals. Philosophical Transactions of the Royal Society of London B, 352, 457-468.

Jones RJ, Hoegh-Guldberg O, Larkum AWD, Schreiber U (1998) Temperature-induced bleaching of corals begins with impairment of the $\mathrm{CO}_{2}$ fixation mechanism in zooxanthellae. Plant, Cell and Environment, 21, 1219-1230.

Judson OP, Normark BB (1996) Ancient asexual scandals. Trends in Ecology and Evolution, 11, 41-46.

Kawaguti S (1944) On the physiology of reef corals. VII. Zooxanthella of the reef corals is Gymnodinium sp., Dinoflagellata; its culture in vitro. Palao Tropical Biology Station Studies, 2, 675-679.

Keshavmurthy S, Hsu C-M, Kuo C-Y et al. (2012) Symbiont communities and host genetic structure of the brain coral Platygyra verweyi, at the outlet of a nuclear power plant and adjacent areas. Molecular Ecology, 21, 4393-4407.

Kevin MJ, Hall WT, McLaughlin JJA, Zahl PA (1969) Symbiodinium microadriaticum Freudenthal, a revised taxonomic description, ultrastructure. Journal of Phycology, $\mathbf{5}$, 341-350.

Kim S, Bachvaroff TR, Handy SM, Delwiche CF (2011) Dynamics of actin evolution in dinoflagellates. Molecular Biology and Evolution, 28, 1469-1480.

Kinzie RA III, Chee GS (1979) The effect of different zooxanthellae on the growth of experimentally reinfected hosts. The Biological Bulletin, 156, 315-327.

Kinzie RA III, Takayama M, Santos SR, Coffroth MA (2001) The adaptive bleaching hypothesis: experimental tests of critical assumptions. The Biological Bulletin, 200, 5158 .

Kirk NL, Ward JR, Coffroth MA (2005) Stable Symbiodinium composition in the sea fan Gorgonia ventalina during temperature and disease stress. The Biological Bulletin, 209, 227-234. 
Kirk NL, Andras JP, Harvell CD, Santos SR, Coffroth MA (2009) Population structure of Symbiodinium sp. associated with the common sea fan, Gorgonia ventalina, in the Florida Keys across distance, depth, and time. Marine Biology, 156, 1609-1623.

Kirtman B, Power SB, Adedoyin JA et al. (2013) Near-term climate change: projections and predictability. In: Climate Change 2013: The Physical Science Basis. Contribution of Working Group I to the Fifth Assessment Report of the Intergovernmental Panel on Climate Change (eds Stocker TF, Qin D, Plattner G-K, et al.). Cambridge University Press, Cambridge.

Kleypas JA, McManus JW, Meñez LAB (1999) Environmental limits to coral reef development: where do we draw the line? American Zoologist, 39, 146-159.

Knowlton N (2001) Coral reef biodiversity-habitat size matters. Science, 292, 1493-1495.

Koike K, Jimbo M, Sakai R et al. (2004) Octocoral chemical signaling selects and controls dinoflagellate symbionts. The Biological Bulletin, 207, 80-86.

Kopp C, Pernice M, Domart-Coulon I et al. (2013) Highly dynamic cellular-level response of symbiotic coral to a sudden increase in environmental nitrogen. mBio, 4, e00052-13.

Koumandou VL, Howe CJ (2007) The copy number of chloroplast gene minicircles changes dramatically with growth phase in the dinoflagellate Amphidinium operculatum. Protist, 158, 89-103.

Krueger T, Gates RD (2012) Cultivating endosymbionts - Host environmental mimics support the survival of Symbiodinium C15 ex hospite. Journal of Experimental Marine Biology and Ecology, 413, 169-176.

LaJeunesse TC (2001) Investigating the biodiversity, ecology, and phylogeny of endosymbiotic dinoflagellates in the genus Symbiodinium using the ITS region: in search of a "species" level marker. Journal of Phycology, 37, 866-880.

LaJeunesse TC (2002) Diversity and community structure of symbiotic dinoflagellates from Caribbean coral reefs. Marine Biology, 141, 387-400.

LaJeunesse TC (2005) "Species" radiations of symbiotic dinoflagellates in the Atlantic and Indo-Pacific since the Miocene-Pliocene transition. Molecular Biology and Evolution, 22, 570-581.

LaJeunesse TC, Pinzón JH (2007) Screening intragenomic rDNA for dominant variants can provide a consistent retrieval of evolutionarily persistent ITS (rDNA) sequences. Molecular Phylogenetics and Evolution, 45, 417-422.

LaJeunesse TC, Trench RK (2000) Biogeography of two species of Symbiodinium (Freudenthal) inhabiting the intertidal sea anemone Anthopleura elegantissima (Brandt). The Biological Bulletin, 199, 126-134. 
LaJeunesse TC, Thornhill DJ (2011) Improved resolution of reef-coral endosymbiont (Symbiodinium) species diversity, ecology, and evolution through $p s b A$ non-coding region genotyping. PLOS ONE, 6, e29013.

LaJeunesse TC, Loh WKW, van Woesik R et al. (2003) Low symbiont diversity in southern Great Barrier Reef corals, relative to those of the Caribbean. Limnology and Oceanography, 48, 2046-2054.

LaJeunesse TC, Thornhill DJ, Cox EF et al. (2004) High diversity and host specificity observed among symbiotic dinoflagellates in reef coral communities from Hawaii. Coral Reefs, 23, 596-603.

LaJeunesse TC, Lambert G, Andersen RA, Coffroth MA, Galbraith DW (2005) Symbiodinium (Pyrrhophyta) genome sizes (DNA content) are smallest among dinoflagellates. Journal of Phycology, 41, 880-886.

LaJeunesse TC, Bonilla HR, Warner ME et al. (2008) Specificity and stability in high latitude eastern Pacific coral-algal symbioses. Limnology and Oceanography, 53, 719727.

LaJeunesse TC, Smith RT, Finney J, Oxenford H (2009) Outbreak and persistence of opportunistic symbiotic dinoflagellates during the 2005 Caribbean mass coral "bleaching" event. Proceedings of the Royal Society B, 276, 4139-4148.

LaJeunesse TC, Pettay DT, Sampayo EM et al. (2010a) Long-standing environmental conditions, geographic isolation and host-symbiont specificity influence the relative ecological dominance and genetic diversification of coral endosymbionts in the genus Symbiodinium. Journal of Biogeography, 37, 785-800.

LaJeunesse TC, Smith R, Walther M et al. (2010b) Host-symbiont recombination versus natural selection in the response of coral-dinoflagellate symbioses to environmental disturbance. Proceedings of the Royal Society B, 277, 2925-2934.

LaJeunesse TC, Parkinson JE, Reimer JD (2012) A genetics-based description of Symbiodinium minutum sp. nov. and S. psygmophilum sp. nov. (Dinophyceae), two dinoflagellates symbiotic with cnidaria. Journal of Phycology, 48, 1380-1391.

LaJeunesse TC, Wham DC, Pettay DT et al. (2014) Ecologically differentiated stress-tolerant endosymbionts in the dinoflagellate genus Symbiodinium (Dinophyceae) Clade D are different species. Phycologia, 53, 305-319.

Law R, Lewis DH (1983) Biotic environments and the maintenance of sex-some evidence from mutualistic symbioses. Biological Journal of the Linnean Society, 20, 249-276.

Lesser MP (1996) Elevated temperatures and ultraviolet radiation cause oxidative stress and inhibit photosynthesis in symbiotic dinoflagellates. Limnology and Oceanography, 41, 271-283.

Lesser MP (1997) Oxidative stress causes coral bleaching during exposure to elevated temperatures. Coral Reefs, 16, 187-192. 
Lesser MP (2006) Oxidative stress in marine environments: biochemistry and physiological ecology. Annual Review of Physiology, 68, 253-278.

Lesser MP, Shick JM (1989) Effects of irradiance and ultraviolet radiation on photoadaptation in the zooxanthellae of Aiptasia pallida: primary production, photoinhibition, and enzymic defenses against oxygen toxicity. Marine Biology, 102, 243-255.

Lesser MP, Farrell JH (2004) Exposure to solar radiation increases damage to both host tissues and algal symbionts of corals during thermal stress. Coral Reefs, 23, 367-377.

Lesser MP, Stochaj WR, Tapley DW, Shick JM (1990) Bleaching in coral reef anthozoans: effects of irradiance, ultraviolet radiation, and temperature on the activities of protective enzymes against active oxygen. Coral Reefs, 8, 225-232.

Lewis CL, Coffroth MA (2004) The acquisition of exogenous algal symbionts by an octocoral after bleaching. Science, 304, 1490-1492.

Lewontin RC, Birch LC (1966) Hybridization as a source of variation for adaptation to new environments. Evolution, 20, 315-336.

Lexer C, Welch ME, Raymond O, Rieseberg LH (2003) The origin of ecological divergence in Helianthus paradoxus (Asteraceae): selection on transgressive characters in a novel hybrid habitat. Evolution, 57, 1989-2000.

Litaker WR, Vandersea MW, Kibler SR et al. (2007) Recognizing dinoflagellate species using ITS rDNA sequences. Journal of Phycology, 43, 344-355.

Little AF, van Oppen MJH, Willis BL (2004) Flexibility in algal endosymbioses shapes growth in reef corals. Science, 304, 1492-1494.

Littman RA, van Oppen MJH, Willis BL (2008) Methods for sampling free-living Symbiodinium (zooxanthellae) and their distribution and abundance at Lizard Island (Great Barrier Reef). Journal of Experimental Marine Biology and Ecology, 364, 48-53.

Lobban CS, Schefter M, Simpson AGB et al. (2002) Maristentor dinoferus n. gen., n. sp., a giant heterotrich ciliate (Spirotrichea: Heterotrichida) with zooxanthellae, from coral reefs on Guam, Mariana Islands. Marine Biology, 140, 411-423.

Loh WKW, Loi T, Carter D, Hoegh-Guldberg O (2001) Genetic variability of the symbiotic dinoflagellates from the wide ranging coral species Seriatopora hystrix and Acropora longicyathus in the Indo-West Pacific. Marine Ecology Progress Series, 222, 97-107.

Loram JE, Boonham N, O'Toole P, Trapido-Rosenthal HG, Douglas AE (2007a) Molecular quantification of symbiotic dinoflagellate algae of the genus Symbiodinium. The Biological Bulletin, 212, 259-268.

Loram JE, Trapido-Rosenthal HG, Douglas AE (2007b) Functional significance of genetically different symbiotic algae Symbiodinium in a coral reef symbiosis. Molecular Ecology, 16, 4849-4857. 
Lupínková L, Komenda J (2004) Oxidative modifications of the photosystem II D1 protein by reactive oxygen species: from isolated protein to cyanobacterial cells.

Photochemistry and Photobiology, 79, 152-162.

Macdonald AHH, Sampayo EM, Ridgway T, Schleyer MH (2008) Latitudinal symbiont zonation in Stylophora pistillata from southeast Africa. Marine Biology, 154, 209-217.

Magalon H, Baudry E, Husté A, Adjeroud M, Veuille M (2006) High genetic diversity of the symbiotic dinoflagellates in the coral Pocillopora meandrina from the South Pacific. Marine Biology, 148, 913-922.

Malinowski DP, Belesky DP (2000) Adaptations of endophyte-infected cool-season grasses to environmental stresses: mechanisms of drought and mineral stress tolerance. Crop Science, 40, 923-940.

Mallet J (2005) Hybridization as an invasion of the genome. Trends in Ecology and Evolution, 20, 229-237.

Mallet J (2007) Hybrid speciation. Nature, 446, 279-283.

Markell DA, Trench RK (1993) Macromolecules exuded by symbiotic dinoflagellates in culture: amino acid and sugar composition. Journal of Phycology, 29, 64-68.

Maynard Smith J (1998) Evolutionary Genetics (2nd edition). Oxford University Press, Oxford.

Mayr E (1942) Systematics and the Origin of Species. Columbia University Press, New York.

Mayr E (1963) Animal Species and Evolution. Harvard University Press, Cambridge MA.

Mayr E (1999) Understanding evolution. Trends in Ecology and Evolution, 14, 372-373.

McNally KL, Govind NS, Thomé PE, Trench RK (1994) Small subunit ribosomal DNA sequence analysis and reconstruction of the inferred phylogeny among symbiotic dinoflagellates (Pyrrophyta). Journal of Phycology, 30, 316-329.

Mieog JC, van Oppen MJH, Cantin NE, Stam WT, Olsen JL (2007) Real-time PCR reveals a high incidence of Symbiodinium clade D at low levels in four scleractinian corals across the Great Barrier Reef: implications for symbiont shuffling. Coral Reefs, 26, 449-457.

Mieog JC, van Oppen MJH, Berkelmans R, Stam WT, Olsen JL (2009) Quantification of algal endosymbionts (Symbiodinium) in coral tissue using real-time PCR. Molecular Ecology Resources, 9, 74-82.

Miller KJ, Ayre DJ (2004) The role of sexual and asexual reproduction in structuring high latitude populations of the reef coral Pocillopora damicornis. Heredity, 92, 557-568.

Miranda LN, Zhuang Y, Zhang H, Lin S (2012) Phylogenetic analysis guided by intragenomic SSU rDNA polymorphism refines classification of "Alexandrium tamarense" species complex. Harmful Algae, 16, 35-48. 
Montalvo AM, Ellstrand NC (2000) Transplantation of the subshrub Lotus scoparius: testing the home-site advantage hypothesis. Conservation Biology, 14, 1034-1045.

Moon CD, Craven KD, Leuchtmann A, Clement SL, Schardl CL (2004) Prevalence of interspecific hybrids amongst asexual fungal endophytes of grasses. Molecular Ecology, 13, 1455-1467.

Moore RB (2003) Highly organized structure in the non-coding region of the $p s b A$ minicircle from clade C Symbiodinium. International Journal of Systematic and Evolutionary Microbiology, 53, 1725-1734.

Morrell PL, Rieseberg LH (1998) Molecular tests of the proposed diploid hybrid origin of Gilia achilleifolia (Polemoniaceae). American Journal of Botany, 85, 1439-1453.

Muller HJ (1964) The relation of recombination to mutational advance. Mutation Research, 1, 2-9.

Muller-Parker G (1984) Photosynthesis-irradiance responses and photosynthetic periodicity in the sea anemone Aiptasia pulchella and its zooxanthellae. Marine Biology, 82, 225232.

Muller-Parker G, D'Elia CF (1997) Interactions between corals and their symbiotic algae. In: Life and Death of Coral Reefs (ed Birkeland C), pp. 96-113. Chapman and Hall, New York.

Muller-Parker G, McCloskey LR, Hoegh-Guldberg O, McAuley PJ (1994) Effect of ammonium enrichment on animal and algal biomass of the coral Pocillopora damicornis. Pacific Science, 48, 273-283.

Muscatine L (1990) The role of symbiotic algae in carbon and energy flux in reef corals. In: Ecosystems of the World, 25: Coral Reefs (ed Dubinsky Z), pp. 75-87. Elsevier, Amsterdam.

Muscatine L, Porter JW (1977) Reef corals: mutualistic symbioses adapted to nutrient-poor environments. BioScience, 27, 454-460.

Nason JD, Ellstrand NC (1993) Estimating the frequencies of genetically distinct classes of individuals in hybridized populations. Journal of Heredity, 84, 1-12.

Nei M, Rooney AP (2005) Concerted and birth-and-death evolution of multigene families. Annual Review of Genetics, 39, 121-152.

Newcombe G, Stirling B, McDonald S, Bradshaw HD (2000) Melampsora $\times$ columbiana, a natural hybrid of M. medusae and M. occidentalis. Mycological Research, 3, 261-274.

Nybakken JW, Bertness MD (2005) Marine Biology: An Ecological Approach (6th edition). Pearson/Benjamin Cummings, San Francisco.

Nyholm S V, McFall-Ngai MJ (2004) The winnowing: establishing the squid-vibrio symbiosis. Nature Reviews Microbiology, 2, 632-642. 
Nyström M (2006) Redundancy and response diversity of functional groups: implications for the resilience of coral reefs. Ambio, 35, 30-35.

Odum HT, Odum EP (1955) Trophic structure and productivity of a windward coral reef community on Eniwetok atoll. Ecological Monographs, 25, 291-320.

Ogden JC (1988) The influence of adjacent systems on the structure and function of coral reefs. Proceedings of the 6th International Coral Reef Symposium, 1, 123-129.

Oliver JK (1984) Intra-colony variation in the growth of Acropora formosa: extension rates and skeletal structure of white (zooxanthellae-free) and brown-tipped branches. Coral Reefs, 3, 139-147.

Oliver TA, Palumbi SR (2011) Many corals host thermally resistant symbionts in hightemperature habitat. Coral Reefs, 30, 241-250.

Omilian AR, Cristescu MEA, Dudycha JL, Lynch M (2006) Ameiotic recombination in asexual lineages of Daphnia. Proceedings of the National Academy of Sciences of the United States of America, 103, 18638-18643.

Van Oppen MJH, Gates RD (2006) Conservation genetics and the resilience of reef-building corals. Molecular Ecology, 15, 3863-3883.

Van Oppen MJH, Palstra FP, Piquet AM-T, Miller DJ (2001) Patterns of coral-dinoflagellate associations in Acropora: significance of local availability and physiology of Symbiodinium strains and host-symbiont selectivity. Proceedings of the Royal Society of London B, 268, 1759-1767.

Van Oppen MJH, Souter P, Howells EJ, Heyward A, Berkelmans R (2011) Novel genetic diversity through somatic mutations: fuel for adaptation of reef corals? Diversity, $\mathbf{3}$, $405-423$.

Padilla-Gamiño JL, Pochon X, Bird C, Concepcion GT, Gates RD (2012) From parent to gamete: vertical transmission of Symbiodinium (Dinophyceae) ITS2 sequence assemblages in the reef building coral Montipora capitata. PLoS ONE, 7, e38440.

Palumbi SR, Barshis DJ, Traylor-Knowles N, Bay RA (2014) Mechanisms of reef coral resistance to future climate change. Science, 344, 895-898.

Pandolfi JM, Connolly SR, Marshall DJ, Cohen AL (2011) Projecting coral reef futures under global warming and ocean acidification. Science, 333, 418-422.

Parrow MW, Burkholder JM (2003a) Reproduction and sexuality in Pfiesteria shumwayae (Dinophyceae). Journal of Phycology, 39, 697-711.

Parrow MW, Burkholder JM (2003b) Estuarine heterotrophic cryptoperidiniopsoids (Dinophyceae): life cycle and culture studies. Journal of Phycology, 39, 678-696.

Parrow MW, Burkholder JM (2004) The sexual life cycles of Pfiesteria piscicida and Cryptoperidiniopsoids (Dinophyceae). Journal of Phycology, 40, 664-673. 
Pasternak Z, Blasius B, Abelson A, Achituv Y (2006) Host-finding behaviour and navigation capabilities of symbiotic zooxanthellae. Coral Reefs, 25, 201-207.

Paulay G (1997) Diversity and distribution of reef organisms. In: Life and Death of Coral Reefs (ed Birkeland C), pp. 298-345. Chapman and Hall, New York.

Pawlowski J, Holzmann M, Fahrni JF, Pochon X, Lee JJ (2001) Molecular identification of algal endosymbionts in large miliolid Foraminifera: 2. Dinoflagellates. The Journal of Eukaryotic Microbiology, 48, 368-373.

Pawlowski J, Audic S, Adl S et al. (2012) CBOL protist working group: barcoding eukaryotic richness beyond the animal, plant, and fungal kingdoms. PLoS Biology, 10, e1001419.

Paxton CW, Davy SK, Weis VM (2013) Stress and death of cnidarian host cells play a role in cnidarian bleaching. The Journal of Experimental Biology, 216, 2813-2820.

Pearse VB, Muscatine L (1971) Role of symbiotic algae (zooxanthellae) in coral calcification. The Biological Bulletin, 141, 350-363.

Peterson A, John H, Koch E, Peterson J (2004) A molecular phylogeny of the genus Gagea (Liliaceae) in Germany inferred from non-coding chloroplast and nuclear DNA sequences. Plant Systematics and Evolution, 245, 145-162.

Pettay DT, LaJeunesse TC (2007) Microsatellites from clade B Symbiodinium spp. specialized for Caribbean corals in the genus Madracis. Molecular Ecology Notes, 7, $1271-1274$.

Pettay DT, LaJeunesse TC (2009) Microsatellite loci for assessing genetic diversity, dispersal and clonality of coral symbionts in "stress-tolerant" clade D Symbiodinium. Molecular Ecology Resources, 9, 1022-1025.

Pettay DT, Wham DC, Pinzón JH, LaJeunesse TC (2011) Genotypic diversity and spatialtemporal distribution of Symbiodinium clones in an abundant reef coral. Molecular Ecology, 20, 5197-5212.

Pfiester LA (1989) Dinoflagellate sexuality. In: International Review of Cytology: A Survey of Cell Biology, Volume 114 (eds Bourne GH, Jeon KW, Friedlander M), pp. 249-270. Academic Press, New York.

Pfiester LA, Anderson DM (1987) Dinoflagellate reproduction. In: The Biology of Dinoflagellates (ed Taylor FJR), pp. 611-648. Blackwell Scientific, London.

Pinzón JH, Devlin-Durante MK, Weber MX, Baums IB, LaJeunesse TC (2011) Microsatellite loci for Symbiodinium A3 (S. fitti) a common algal symbiont among Caribbean Acropora (stony corals) and Indo-Pacific giant clams (Tridacna). Conservation Genetics Resources, 3, 45-47.

Pochon X, Gates RD (2010) A new Symbiodinium clade (Dinophyceae) from soritid foraminifera in Hawai'i. Molecular Phylogenetics and Evolution, 56, 492-497. 
Pochon X, Pawlowski J, Zaninetti L, Rowan R (2001) High genetic diversity and relative specificity among Symbiodinium-like endosymbiotic dinoflagellates in soritid foraminiferans. Marine Biology, 139, 1069-1078.

Pochon X, Stat M, Takabayashi M et al. (2010) Comparison of endosymbiotic and free-living Symbiodinium (Dinophyceae) diversity in a Hawaiian reef environment. Journal of Phycology, 46, 53-65.

Pochon X, Putnam HM, Burki F, Gates RD (2012) Identifying and characterizing alternative molecular markers for the symbiotic and free-living dinoflagellate genus Symbiodinium. PLOS ONE, 7, e29816.

Pochon X, Putnam HM, Gates RD (2014) Multi-gene analysis of Symbiodinium dinoflagellates: a perspective on rarity, symbiosis, and evolution. PeerJ, 2, e394.

Putnam HM, Stat M, Pochon X, Gates RD (2012) Endosymbiotic flexibility associates with environmental sensitivity in scleractinian corals. Proceedings of the Royal Society B, 279, 4352-4361.

Quigley KM, Davies SW, Kenkel CD et al. (2014) Deep-sequencing method for quantifying background abundances of Symbiodinium types: exploring the rare Symbiodinium biosphere in reef-building corals. PLoS ONE, 9, e94297.

Quinn GP, Keough MJ (2002) Experimental Design and Data Analysis for Biologists. Cambridge University Press, Cambridge.

R Development Core Team (2011) R: A Language and Environment for Statistical Computing.

Ragni M, Airs RL, Hennige SJ et al. (2010) PSII photoinhibition and photorepair in Symbiodinium (Pyrrhophyta) differs between thermally tolerant and sensitive phylotypes. Marine Ecology Progress Series, 406, 57-70.

Rasch D, Mašata O (2006) Methods of variance component estimation. Czech Journal of Animal Science, 51, 227-235.

Renger G, Völker M, Eckert HJ et al. (1989) On the mechanism of photosystem II deterioration by UV-B irradiation. Photochemistry and Photobiology, 49, 97-105.

Rhymer JM, Simberloff D (1996) Extinction by hybridization and introgression. Annual Review of Ecology and Systematics, 27, 83-109.

Richmond RH, Hunter CL (1990) Reproduction and recruitment of corals: comparisons among the Caribbean, the Tropical Pacific, and the Red Sea. Marine Ecology Progress Series, 60, 185-203.

Rieseberg LH (1991) Homoploid reticulate evolution in Helianthus (Asteraceae): evidence from ribosomal genes. American Journal of Botany, 78, 1218-1237. 
Rieseberg LH (1997) Hybrid origins of plant species. Annual Review of Ecology and Systematics, 28, 359-389.

Rieseberg L, Soltis DE (1991) Phylogenetic consequences of cytoplasmic gene flow in plants. Evolutionary Trends in Plants, 5, 65-84.

Rieseberg LH, Whitton J, Linder CR (1996) Molecular marker incongruence in plant hybrid zones and phylogenetic trees. Acta Botanica Neerlandica, 45, 243-262.

Rieseberg LH, Archer MA, Wayne RK (1999) Transgressive segregation, adaptation and speciation. Heredity, 83, 363-372.

Rieseberg LH, Baird SJE, Gardner KA (2000) Hybridization, introgression, and linkage evolution. Plant Molecular Biology, 42, 205-224.

Rieseberg LH, Raymond O, Rosenthal DM et al. (2003) Major ecological transitions in wild sunflowers facilitated by hybridization. Science, 301, 1211-1216.

Ritchie RJ (2006) Consistent sets of spectrophotometric chlorophyll equations for acetone, methanol and ethanol solvents. Photosynthesis Research, 89, 27-41.

Ritchie RJ, Grant AJ, Eltringham K, Hinde R (1997) Clotrimazole, a model compound for the host release factor of the coral Plesiastrea versipora. Functional Plant Biology, 24, 283290.

Rodriguez-Lanetty M (2003) Evolving lineages of Symbiodinium-like dinoflagellates based on ITS1 rDNA. Molecular Phylogenetics and Evolution, 28, 152-168.

Rodriguez-Lanetty M, Loh W, Carter D, Hoegh-Guldberg O (2001) Latitudinal variability in symbiont specificity within the widespread scleractinian coral Plesiastrea versipora. Marine Biology, 138, 1175-1181.

Rohwer F, Breitbart M, Jara J, Azam F, Knowlton N (2001) Diversity of bacteria associated with the Caribbean coral Montastraea franksi. Coral Reefs, 20, 85-91.

Rohwer F, Seguritan V, Azam F, Knowlton N (2002) Diversity and distribution of coralassociated bacteria. Marine Ecology Progress Series, 243, 1-10.

Rosenberg E, Ben-Haim Y (2002) Microbial diseases of corals and global warming. Environmental Microbiology, 4, 318-326.

Rowan R (1998) Diversity and ecology of zooxanthellae on coral reefs. Journal of Phycology, 34, 407-417.

Rowan R (2004) Thermal adaptation in reef coral symbiosis. Nature, 430, 742.

Rowan R, Powers DA (1991a) Molecular genetic identification of symbiotic dinoflagellates (zooxanthellae). Marine Ecology Progress Series, 71, 65-73. 
Rowan R, Powers DA (1991b) A molecular genetic classification of zooxanthellae and the evolution of animal-algal symbioses. Science, 251, 1348-1351.

Rowan R, Powers DA (1992) Ribosomal RNA sequences and the diversity of symbiotic dinoflagellates (zooxanthellae). Proceedings of the National Academy of Sciences of the United States of America, 89, 3639-3643.

Rowan R, Knowlton N (1995) Intraspecific diversity and ecological zonation in coral-algal symbiosis. Proceedings of the National Academy of Sciences of the United States of America, 92, 2850-2853.

Rowan R, Knowlton N, Baker A, Jara J (1997) Landscape ecology of algal symbionts creates variation in episodes of coral bleaching. Nature, 388, 265-269.

Saari S, Faeth SH (2012) Hybridization of Neotyphodium endophytes enhances competitive ability of the host grass. New Phytologist, 195, 231-236.

Sachs JL, Wilcox TP (2006) A shift to parasitism in the jellyfish symbiont Symbiodinium microadriaticum. Proceedings of the Royal Society B, 273, 425-429.

Sampayo EM, Franceschinis L, Hoegh-Guldberg O, Dove S (2007) Niche partitioning of closely related symbiotic dinoflagellates. Molecular Ecology, 16, 3721-3733.

Sampayo EM, Ridgway T, Bongaerts P, Hoegh-Guldberg O (2008) Bleaching susceptibility and mortality of corals are determined by fine-scale differences in symbiont type. Proceedings of the National Academy of Sciences of the United States of America, 105, 10444-10449.

Sampayo EM, Dove S, LaJeunesse TC (2009) Cohesive molecular genetic data delineate species diversity in the dinoflagellate genus Symbiodinium. Molecular Ecology, 18, $500-519$.

Sang T, Crawford DJ, Stuessy TF (1995) Documentation of reticulate evolution in peonies (Paeonia) using internal transcribed spacer sequences of nuclear ribosomal DNA: implications for biogeography and concerted evolution. Proceedings of the National Academy of Sciences of the United States of America, 92, 6813-6817.

Santos SR, Coffroth MA (2003) Molecular genetic evidence that dinoflagellates belonging to the genus Symbiodinium Freudenthal are haploid. The Biological Bulletin, 204, 10-20.

Santos SR, Taylor DJ, Coffroth MA (2001) Genetic comparisons of freshly isolated versus cultured symbiotic dinoflagellates: implications for extrapolating to the intact symbiosis. Journal of Phycology, 37, 900-912.

Santos SR, Gutierrez-Rodriguez C, Coffroth MA (2003a) Phylogenetic identification of symbiotic dinoflagellates via length heteroplasmy in domain $\mathrm{V}$ of chloroplast large subunit (cp23S)-ribosomal DNA sequences. Marine Biotechnology, 5, 130-140.

Santos SR, Gutiérrez-Rodríguez C, Lasker HR, Coffroth MA (2003b) Symbiodinium sp. associations in the gorgonian Pseudopterogorgia elisabethae in the Bahamas: high 
levels of genetic variability and population structure in symbiotic dinoflagellates. Marine Biology, 143, 111-120.

Santos SR, Shearer TL, Hannes AR, Coffroth MA (2004) Fine-scale diversity and specificity in the most prevalent lineage of symbiotic dinoflagellates (Symbiodinium, Dinophyceae) of the Caribbean. Molecular Ecology, 13, 459- 469.

Santos SR, Taylor DJ, Kinzie RA III et al. (2002) Molecular phylogeny of symbiotic dinoflagellates inferred from partial chloroplast large subunit (23S)-rDNA sequences. Molecular Phylogenetics and Evolution, 23, 97-111.

Savage AM, Trapido-Rosenthal H, Douglas AE (2002) On the functional significance of molecular variation in Symbiodinium, the symbiotic algae of Cnidaria: photosynthetic response to irradiance. Marine Ecology Progress Series, 244, 27-37.

Schardl CL, Clay K (1997) Evolution of mutualistic endophytes from plant pathogens. In: The Mycota V: Plant Relationships Part B (eds Carroll GC, Tudzynski P), pp. 221-238. Springer-Verlag, Berlin.

Schardl CL, Craven KD (2003) Interspecific hybridization in plant-associated fungi and oomycetes: a review. Molecular Ecology, 12, 2861-2873.

Schardl CL, Liu J-S, White JF Jr et al. (1991) Molecular phylogenetic relationships of nonpathogenic grass mycosymbionts and clavicipitaceous plant pathogens. Plant Systematics and Evolution, 178, 27-41.

Schardl CL, Leuchtmann A, Tsai H-F et al. (1994) Origin of a fungal symbiont of perennial ryegrass by interspecific hybridization of a mutualist with the ryegrass choke pathogen, Epichloë typhina. Genetics, 136, 1307-1317.

Schiffers K, Bourne EC, Lavergne S, Thuiller W, Travis JMJ (2013) Limited evolutionary rescue of locally adapted populations facing climate change. Philosophical Transactions of the Royal Society of London B, 368, 20120083.

Schoenberg DA, Trench RK (1976) Specificity of symbioses between marine cnidarians and zooxanthellae. In: Coelenterate Ecology and Behavior (ed Mackie GO), pp. 423-432. Plenum Press, New York.

Schoenberg DA, Trench RK (1980a) Genetic variation in Symbiodinium (=Gymnodinium) microadriaticum Freudenthal, and specificity in its symbiosis with marine invertebrates. I. Isoenzyme and soluble protein patterns of axenic cultures of Symbiodinium microadriaticum. Proceedings of the Royal Society of London. Series B, Biological Sciences, 207, 405-427.

Schoenberg DA, Trench RK (1980b) Genetic variation in Symbiodinium (=Gymnodinium) microadriaticum Freudenthal, and specificity in its symbiosis with marine invertebrates. II. Morphological variation in Symbiodinium microadriaticum. Proceedings of the Royal Society of London. Series B, Biological Sciences, 207, 429-444. 
Schoenberg DA, Trench RK (1980c) Genetic variation in Symbiodinium (=Gymnodinium) microadriaticum Freudenthal, and specificity in its symbiosis with marine invertebrates. III. Specificity and infectivity of Symbiodinium microadriaticum. Proceedings of the Royal Society of London. Series B, Biological Sciences, 207, 445-460.

Schön I, Martens K (1998) DNA repair in ancient asexuals - a new solution to an old problem? Journal of Natural History, 32, 943-948.

Seehausen O (2004) Hybridization and adaptive radiation. Trends in Ecology and Evolution, 19, 198-207.

Seutin G, White BN, Boag PT (1991) Preservation of avian blood and tissue samples for DNA analyses. Canadian Journal of Zoology, 69, 82-90.

Sheppard CRC, Davy SK, Pilling GM (2009) The Biology of Coral Reefs. Oxford University Press, New York.

Shoguchi E, Shinzato C, Kawashima T et al. (2013) Draft assembly of the Symbiodinium minutum nuclear genome reveals dinoflagellate gene structure. Current Biology, 23, 1399-1408.

Silverstein RN, Correa AMS, LaJeunesse TC, Baker AC (2011) Novel algal symbiont (Symbiodinium spp.) diversity in reef corals of Western Australia. Marine Ecology Progress Series, 422, 63-75.

Silverstein RN, Correa AMS, Baker AC (2012) Specificity is rarely absolute in coral-algal symbiosis: implications for coral response to climate change. Proceedings of the Royal Society B, 279, 2609-2618.

Simpson GG (1953) The Major Features of Evolution. Columbia University Press, New York.

Small AM, Adey WH, Spoon D (1998) Are current estimates of coral reef biodiversity too low? The view through the window of a microcosm. Atoll Research Bulletin, 458, 1-20.

Small RL, Cronn RC, Wendel JF (2004) Use of nuclear genes for phylogeny reconstruction in plants. Australian Systematic Biology, 17, 145-170.

Smith S (1978) Coral-reef area and the contributions of reefs to processes and resources of the world's oceans. Nature, 273, 225-226.

Soltis PS, Soltis DE, Doyle JJ (1992) Molecular Systematics of Plants. Chapman and Hall, New York.

Spinaze KA, Smith SDA, Simpson RD (1996) The effect of depth and wave exposure on the density and porosity of Pocillopora damicornis in the Solitary Islands Marine Reserve, New South Wales. Proceedings of The Great Barrier Reef, Science, Use and Management Conference, 2, 72-76. 
Stambler N (2011) Zooxanthellae: the yellow symbionts inside animals. In: Coral Reefs: An Ecosystem in Transition (eds Dubinsky Z, Stambler N), pp. 87-106. Springer, New York.

Stanley GD Jr, Swart PK (1995) Evolution of the coral-zooxanthellae symbiosis during the Triassic: a geochemical approach. Paleobiology, 21, 179-199.

Starzak DE, Quinnell RG, Nitschke MR, Davy SK (2014) The influence of symbiont type on photosynthetic carbon flux in a model cnidarian-dinoflagellate symbiosis. Marine Biology, 161, 711-724.

Stat M, Carter D, Hoegh-Guldberg O (2006) The evolutionary history of Symbiodinium and scleractinian hosts - Symbiosis, diversity, and the effect of climate change. Perspectives in Plant Ecology, Evolution and Systematics, 8, 23-43.

Stat M, Loh WKW, Hoegh-Guldberg O, Carter DA (2008a) Symbiont acquisition strategy drives host-symbiont associations in the southern Great Barrier Reef. Coral Reefs, 27, $763-772$.

Stat M, Morris E, Gates RD (2008b) Functional diversity in coral-dinoflagellate symbiosis. Proceedings of the National Academy of Sciences of the United States of America, 105, 9256-9261.

Stat M, Pochon X, Cowie ROM, Gates RD (2009) Specificity in communities of Symbiodinium in corals from Johnston Atoll. Marine Ecology Progress Series, 386, 8396.

Stat M, Bird CE, Pochon X et al. (2011) Variation in Symbiodinium ITS2 sequence assemblages among coral colonies (D Steinke, Ed,). PLoS ONE, 6, e15854.

Stat M, Baker AC, Bourne DG et al. (2012) Molecular delineation of species in the coral holobiont. In: Advances in Marine Biology, Vol 63 (ed Lesser MP), pp. 1-65. Academic Press, Amsterdam.

Stebbins GL Jr (1950) Variation and Evolution in Plants. Columbia University Press, New York.

Steen RG, Muscatine L (1984) Daily budgets of photosynthetically fixed carbon in symbiotic zoanthids. The Biological Bulletin, 167, 477-487.

Steen RG, Muscatine L (1987) Low temperature evokes rapid exocytosis of symbiotic algae by a sea anemone. The Biological Bulletin, 172, 246-263.

Stern RF, Horak A, Andrew RL et al. (2010) Environmental barcoding reveals massive dinoflagellate diversity in marine environments. PLoS ONE, 5, e13991.

Stern RF, Andersen RA, Jameson I et al. (2012) Evaluating the ribosomal internal transcribed spacer (ITS) as a candidate dinoflagellate barcode marker. PLOS ONE, 7, e42780. 
Stimson J, Kinzie RA III (1991) The temporal pattern and rate of release of zooxanthellae from the reef coral Pocillopora damicornis (Linnaeus) under nitrogen-enrichment and control conditions. Journal of Experimental Marine Biology and Ecology, 153, 63-74.

Takabayashi M, Santos SR, Cook CB (2004) Mitochondrial DNA phylogeny of the symbiotic dinoflagellates (Symbiodinium, Dinophyta). Journal of Phycology, 40, 160-164.

Takabayashi M, Adams LM, Pochon X, Gates RD (2011) Genetic diversity of free-living Symbiodinium in surface water and sediment of Hawai'i and Florida. Coral Reefs, 31, 157-167.

Tanaka Y, Miyajima T, Koike I, Hayashibara T, Ogawa H (2006) Translocation and conservation of organic nitrogen within the coral-zooxanthella symbiotic system of Acropora pulchra, as demonstrated by dual isotope-labeling techniques. Journal of Experimental Marine Biology and Ecology, 336, 110-119.

Taylor DL (1969) Identity of zooxanthellae isolated from some Pacific Tridacnidae. Journal of Phycology, 5, 336-340.

Taylor DL (1973) The cellular interactions of algal invertebrate symbiosis. Advances in Marine Biology, 11, 1-56.

Taylor DL (1974) Symbiotic marine algae: taxonomy and biological fitness. In: Symbiosis in the Sea (ed Vernberg WB), pp. 245-262. University of South Carolina Press, Colombia.

Taylor SJ, Arnold M, Martin NH (2009) The genetic architecture of reproductive isolation in Louisiana irises: hybrid fitness in nature. Evolution, 63, 2581-2594.

Tchernov D, Gorbunov MY, de Vargas C et al. (2004) Membrane lipids of symbiotic algae are diagnostic of sensitivity to thermal bleaching in corals. Proceedings of the National Academy of Sciences of the United States of America, 101, 13531-13535.

Templeton AR (1989) The meaning of species and speciation: a genetic perspective. In: Speciation and its Consequences (eds Otte D, Endler JA), pp. 3-27. Sinauer, Sunderland MA.

Tengs T, Dahlberg OJ, Shalchian-Tabrizi K et al. (2000) Phylogenetic analyses indicate that the 19'hexanoyloxy-fucoxanthin-containing dinoflagellates have tertiary plastids of haptophyte origin. Molecular Biology and Evolution, 17, 718-729.

Thomas L, Kendrick GA, Kennington WJ, Richards ZT, Stat M (2014) Exploring Symbiodinium diversity and host specificity in Acropora corals from geographical extremes of Western Australia with 454 amplicon pyrosequencing. Molecular Ecology, DOI: $10.1111 / \mathrm{mec} .12801$.

Thomas MA, Roemer GW, Donlan CJ et al. (2013) Gene tweaking for conservation. Nature, 501, 485-486.

Thornhill DJ, Fitt WK, Schmidt GW (2006a) Highly stable symbioses among western Atlantic brooding corals. Coral Reefs, 25, 515-519. 
Thornhill DJ, LaJeunesse TC, Kemp DW, Fitt WK, Schmidt GW (2006b) Multi-year, seasonal genotypic surveys of coral-algal symbioses reveal prevalent stability or postbleaching reversion. Marine Biology, 148, 711-722.

Thornhill DJ, Doubleday K, Kemp DW, Santos SR (2010) Host hybridization alters specificity of cnidarian-dinoflagellate associations. Marine Ecology Progress Series, 420, 113-123.

Thornhill DJ, LaJeunesse TC, Santos SR (2007) Measuring rDNA diversity in eukaryotic microbial systems: how intragenomic variation, pseudogenes, and PCR artifacts confound biodiversity estimates. Molecular Ecology, 16, 5326-5340.

Thornhill DJ, Xiang Y, Fitt WK, Santos SR (2009) Reef endemism, host specificity and temporal stability in populations of symbiotic dinoflagellates from two ecologically dominant Caribbean corals. PLoS ONE, 4, e6262.

Thornhill DJ, Lewis AM, Wham DC, LaJeunesse TC (2013a) Host-specialist lineages dominate the adaptive radiation of reef coral endosymbionts. Evolution, 68, 352-367.

Thornhill DJ, Xiang Y, Pettay DT, Zhong M, Santos SR (2013b) Population genetic data of a model symbiotic cnidarian system reveal remarkable symbiotic specificity and vectored introductions across ocean basins. Molecular Ecology, 22, 4499-4515.

Tibayrenc M, Kjellberg F, Arnaud J et al. (1991) Are eukaryotic microorganisms clonal or sexual? A population genetics vantage. Proceedings of the National Academy of Sciences of the United States of America, 88, 5129-5133.

Titlyanov EA, Titlyanova T V, Leletkin VA et al. (1996) Degradation of zooxanthellae and regulation of their density in hermatypic corals. Marine Ecology Progress Series, 139, $167-178$.

Toller WW, Rowan R, Knowlton N (2001a) Zooxanthellae of the Montastraea annularis species complex: patterns of distribution of four taxa of Symbiodinium on different reefs and across depths. The Biological Bulletin, 201, 348-359.

Toller WW, Rowan R, Knowlton N (2001b) Repopulation of zooxanthellae in the Caribbean corals Montastraea annularis and $M$. faveolata following experimental and diseaseassociated bleaching. The Biological Bulletin, 201, 360-373.

Tomascik T, Sander F (1985) Effects of eutrophication on reef-building corals. I. Growth rate of the reef-building coral Montastrea annularis. Marine Biology, 87, 143-155.

Tomascik T, Sander F (1987a) Effects of eutrophication on reef-building corals. II. Structure of scleractinian coral communities on fringing reefs, Barbados, West Indies. Marine Biology, 94, 53-75.

Tomascik T, Sander F (1987b) Effects of eutrophication on reef-building corals. III. Reproduction of the reef-building coral Porites porites. Marine Biology, 94, 77-94. 
Tonk L, Bongaerts P, Sampayo EM, Hoegh-Guldberg O (2013) SymbioGBR: a web-based database of Symbiodinium associated with cnidarian hosts on the Great Barrier Reef. BMC Ecology, 13, 7.

Trench RK (1971) The physiology and biochemistry of zooxanthellae symbiotic with marine coelenterates. III. The effect of homogenates of host tissues on the excretion of photosynthetic products in vitro by zooxanthellae from two marine coelenterates. Proceedings of the Royal Society of London. Series B, Biological Sciences, 177, 251264.

Trench RK (1987) Dinoflagellates in non-parasitic symbioses. In: The Biology of Dinoflagellates (ed Taylor FJR), pp. 530-570. Blackwell, Oxford.

Trench RK (1993) Microalgal-invertebrate symbioses: a review. Endocytobiosis and Cell Research, 9, 135-175.

Trench RK (1997) Diversity of symbiotic dinoflagellates and the evolution of microalgalinvertebrate symbiosis. Proceedings of the 8th International Coral Reef Symposium, 2, $1275-1286$.

Trench R (2000) Validation of some currently used invalid names of dinoflagellates. Journal of Phycology, 36, 972.

Trench RK, Blank RJ (1987) Symbiodinium microadriaticum Freudenthal, S. goreauii sp. nov., S. kawagutii sp. nov., and S. pilosum sp. nov.: Gymnodinioid dinoflagellate symbionts of marine invertebrates. Journal of Phycology, 23, 469-481.

Trench RK, Thinh L-V (1995) Gymnodinium linucheae sp. nov.: the dinoflagellate symbiont of the jellyfish Linuche unguiculata. European Journal of Phycology, 30, 149-154.

Tsai H-F, Liu J-S, Staben C et al. (1994) Evolutionary diversification of fungal endophytes of tall fescue grass by hybridization with Epichloë species. Proceedings of the National Academy of Sciences of the United States of America, 91, 2542-2546.

Tsitrone A, Kirkpatrick M, Levin DA (2003) A model for chloroplast capture. Evolution, 57, 1776-1782.

Turpin DH, Dobell PER, Taylor FJR (1978) Sexuality and cyst formation in Pacific strains of the toxic dinoflagellate Gonyaulax tamarensis. Journal of Phycology, 14, 235-238.

Ulstrup KE, van Oppen MJH (2003) Geographic and habitat partitioning of genetically distinct zooxanthellae (Symbiodinium) in Acropora corals on the Great Barrier Reef. Molecular Ecology, 12, 3477-3484.

Ulstrup KE, Berkelmans R, Ralph PJ, van Oppen MJH (2006) Variation in bleaching sensitivity of two coral species across a latitudinal gradient on the Great Barrier Reef: the role of zooxanthellae. Marine Ecology Progress Series, 314, 135-148. 
Ulstrup KE, van Oppen MJH, Kühl M, Ralph PJ (2007) Inter-polyp genetic and physiological characterisation of Symbiodinium in an Acropora valida colony. Marine Biology, 153, 225-234.

Ulstrup KE, Hill R, van Oppen MJH, Larkum AWD, Ralph PJ (2008) Seasonal variation in the photo-physiology of homogeneous and heterogeneous Symbiodinium consortia in two scleractinian corals. Marine Ecology Progress Series, 361, 139-150.

UNESCO (1981) Tenth report of the joint panel on oceanographic tables and standards. Unesco Technical Papers in Marine Science, 36, 1-25.

Van Valen L (1976) Ecological species, multispecies, and oaks. Taxon, 25, 233-239.

Vandermeulen JH, Davis ND, Muscatine L (1972) The effect of inhibitors of photosynthesis on zooxanthellae in corals and other marine invertebrates. Marine Biology, 16, 185-191.

Vanormelingen P, Chepurnov VA, Mann DG, Sabbe K, Vyverman W (2008) Genetic divergence and reproductive barriers among morphologically heterogeneous sympatric clones of Eunotia bilunaris sensu lato (Bacillariophyta). Protist, 159, 73-90.

Venn AA, Loram JE, Trapido-Rosenthal HG, Joyce DA, Douglas AE (2008) Importance of time and place: patterns in abundance of Symbiodinium clades A and B in the tropical sea anemone Condylactis gigantea. The Biological Bulletin, 215, 243-252.

Veron JEN (1995) Corals in Space and Time. University of New South Wales Press, Sydney.

Veron JEN (2000) Corals of the World. Australian Institute of Marine Science, Townsville.

Veron JEN, Done TJ (1979) Corals and coral communities of Lord Howe Island. Australian Journal of Marine and Freshwater Research, 30, 203-236.

Vriesendorp B, Bakker FT (2005) Reconstructing patterns of reticulate evolution in angiosperms: what can we do? Taxon, 54, 593-604.

Wakefield TS, Farmer MA, Kempf SC (2000) Revised description of the fine structure of in situ “zooxanthellae" genus Symbiodinium. The Biological Bulletin, 199, 76-84.

Wakefield TS, Kempf SC (2001) Development of host- and symbiont-specific monoclonal antibodies and confirmation of the origin of the symbiosome membrane in a cnidariandinoflagellate symbiosis. The Biological Bulletin, 200, 127-143.

Wang J-T, Douglas AE (1997) Nutrients, signals, and photosynthate release by symbiotic alge. Plant Physiology, 114, 631-636.

Wang J-T, Douglas AE (1998) Nitrogen recycling or nitrogen conservation in an algainvertebrate symbiosis? The Journal of Experimental Biology, 201, 2445-2453.

Wang XR, Szmidt AE, Lewandowski A, Wang ZR (1990) Evolutionary analysis of Pinus densata Masters, a putative Tertiary hybrid. 1. Allozyme variation. Theoretical and Applied Genetics, 80, 635-640. 
Warner ME, Fitt WK, Schmidt GW (1999) Damage to photosystem II in symbiotic dinoflagellates: A determinant of coral bleaching. Proceedings of the National Academy of Sciences of the United States of America, 96, 8007-8012.

Welch ME, Rieseberg LH (2002) Habitat divergence between a homoploid hybrid sunflower species, Helianthus paradoxus (Asteraceae), and its progenitors. American Journal of Botany, 89, 472-478.

Wendel JF, Schnabel A, Seelanan T (1995) Bidirectional interlocus concerted evolution following allopolyploid speciation in cotton (Gossypium). Proceedings of the National Academy of Sciences of the United States of America, 92, 280-284.

Wham DC, Pettay DT, LaJeunesse TC (2011) Microsatellite loci for the host-generalist "zooxanthella" Symbiodinium trenchi and other Clade D Symbiodinium. Conservation Genetics Resources, 3, 541-544.

Whitehead LF, Douglas AE (2003) Metabolite comparisons and the identity of nutrients translocated from symbiotic algae to an animal host. The Journal of Experimental Biology, 206, 3149-3157.

Wicks LC, Sampayo EM, Gardner JPA, Davy SK (2010) Local endemicity and high diversity characterise high-latitude coral-Symbiodinium partnerships. Coral Reefs, 29, 989-1003.

Wicks LC, Gardner JPA, Davy SK (2012) Host tolerance, not symbiont tolerance, determines the distribution of coral species in relation to their environment at a Central Pacific atoll. Coral Reefs, 31, 389-398.

Wilcox TP (1998) Large-subunit ribosomal RNA systematics of symbiotic dinoflagellates: morphology does not recapitulate phylogeny. Molecular Phylogenetics and Evolution, 10, 436-448.

Wilkerson FP, Kobayashi D, Muscatine L (1988) Mitotic index and size of symbiotic algae in Caribbean Reef corals. Coral Reefs, 7, 29-36.

Wilkinson C, Lindén O, Cesar H et al. (1999) Ecological and socioeconomic impacts of 1998 coral mortality in the Indian Ocean: an ENSO impact and a warning of future change? Ambio, 28, 188-196.

Willis BL, van Oppen MJH, Miller DJ, Vollmer S V, Ayre DJ (2006) The role of hybridization in the evolution of reef corals. Annual Review of Ecology, Evolution, and Systematics, 37, 489-517.

Wright S (1932) The roles of mutation, inbreeding, crossbreeding and selection in evolution. Proceedings of the 6th International Congress of Genetics, 1, 356-366.

Xiang T, Hambleton EA, DeNofrio JC, Pringle JR, Grossman AR (2013) Isolation of clonal axenic strains of the symbiotic dinoflagellate Symbiodinium and their growth and host specificity. Journal of Phycology, 49, 447-458. 
Yachi S, Loreau M (1999) Biodiversity and ecosystem productivity in a fluctuating environment: The insurance hypothesis. Proceedings of the National Academy of Sciences of the United States of America, 96, 1463-1468.

Yamashita H, Suzuki G, Hayashibara T, Koike K (2010) Do corals select zooxanthellae by alternative discharge? Marine Biology, 158, 87-100.

Yellowlees D, Rees TA V, Leggat W (2008) Metabolic interactions between algal symbionts and invertebrate hosts. Plant, Cell and Environment, 31, 679-694.

Zardoya R, Costas E, López-Rodas V, Garrido-Pertierra A, Bautista JM (1995) Revised dinoflagellate phylogeny inferred from molecular analysis of large-subunit ribosomal RNA gene sequences. Journal of Molecular Evolution, 41, 637-645.

Zhang H, Bhattacharya D, Lin S (2005) Phylogeny of dinoflagellates based on mitochondrial cytochrome $b$ and nuclear small subunit rDNA sequence comparisons. Journal of Phycology, 41, 411-420.

Zhang H, Hou Y, Lin S (2006) Isolation and characterization of proliferating cell nuclear antigen from the dinoflagellate Pfiesteria piscicida. Journal of Eukaryotic Microbiology, 53, 142-150. 
Appendix A: Nucleotide sequences and alignments 
Table A.1 Genbank accession numbers for sequences used in rDNA phylogeny (Figure 1.4)

\begin{tabular}{|c|c|c|}
\hline Alphanumeric designation & Species name & Genbank accession number(s) \\
\hline A1 & Symbiodinium microadriaticum & AF333505, U63483 \\
\hline A2 & Symbiodinium pilosum & AF333506, JN558097, AF060894 \\
\hline A3 & 'Symbiodinium fitti' & AF333507, JN558091, KF364601 \\
\hline A4 & Symbiodinium linucheae & AF427465, AY074949, AF333509 \\
\hline $\mathbf{A}_{\text {III }}$ & Symbiodinium natans & AB704015, AB704056, EU315917 \\
\hline B1 & Symbiodinium minutum & AF333511, JN558059, AF060892 \\
\hline B2 & Symbiodinium psygmophilum & AF333512, JN558061 \\
\hline C1 & Symbiodinium goreauii & AF333515, JN558040, JN558041 \\
\hline C3 & & AB 294654, EU786077, FJ529524, JF834208, J834209 \\
\hline C3nt & & FJ529569, FJ529594-FJ529604, FJ529530 \\
\hline C8a & & FJ529563, FJ529612-FJ529619, FJ529526 \\
\hline C33 & & FJ529566, FJ529567, AY258498, AY765400, FJ529532 \\
\hline C33a & & FJ529564, FJ529565, FJ529624- FJ529626, FJ529531 \\
\hline C35a & & FJ529559, FJ529584-FJ529593, FJ529529, EF541146, EU808002, AY258501 \\
\hline C42a & & FJ529561, FJ529634-FJ529655, FJ529525, HQ650837 \\
\hline C78a & & FJ529562, FJ529605-FJ529611, FJ529527, EU808000 \\
\hline C79 & & FJ529560, FJ529570-FJ525581, FJ529528, AY765414, EU807997 \\
\hline C91 & & AJ291519, JN558048 \\
\hline D1a & Symbiodinium trenchii & EU074894, EU074898, EU074903-EU074906, JN558078, KJ019889, KF740689 \\
\hline D1 & 'Symbiodinium glynni' & AF334660, JN558075, JN558076, AF396626-AF396628, JN601885, DQ312315 \\
\hline E1 & Symbiodinium voratum & AF334659, JN558084- JN558086, KF364603- KF364605 \\
\hline F1 & Symbiodinium kawagutii & AF360577, AF333517, JN558066, AF427462 \\
\hline F2 & & AF333516, JN558065, KF740673 \\
\hline $\mathbf{G}$ & & AJ291537 \\
\hline $\mathbf{H}$ & & AJ291513 \\
\hline \multirow[t]{3}{*}{$\mathbf{I}$} & & FN561559 \\
\hline & Polarella glacialis & GQ375263, AB704041, AB704042, FJ939578, AY571373 \\
\hline & Protodinium simplex & JF791031, AY686651, JN558103, AF060900 \\
\hline
\end{tabular}

*Species names in inverted commas are yet to be formally described 
Table A.2 Deposition of novel internal transcribed spacer 2 (ITS2) sequence (chapter 2)

> KJ530690 Symbiodinium sp. C109 5.8S ribosomal RNA gene, partial sequence; internal transcribed spacer 2, complete sequence; and 28S ribosomal RNA gene, partial sequence

AACCAATGGCCTCCTGAACGTGCGTTGCACTCTTGGGATTTCCTGAGAGTACGTCTGCTTCAGTGC TTAACTTGCCCCAACTTTGCAAGCAGGATGTGTTTCTGCCTTGCGTTCTTATGAGCTATTGCCCTCT GAGCCAATGGCTTGTTAATTGCTTGGTTCTTGCAAAATGCTTTGCGCGCTGTTATTCAAGTTTCTAC CTTCGTGGTTTTACTTGAGTGACGCTGCTCATGCTTGCAACCGCTGGGATGCAGGTGCATGCCTCTA GCATGAAGTCAGACAAGTGA

Table A.3 ITS2 sequences used in statistical parsimony network (Figure 2.2)

\begin{tabular}{ll}
\hline Alphanumeric designation & Genbank accession number \\
\hline C1 (Symbiodinium goreauii) & AF333515 \\
C1bb & HM222430 \\
C100 & HM222433 \\
C103 & HM222435 \\
C109 & KJ530690 \\
C118 & HM222440 \\
C3 & GU111863 \\
C3gg & HM222431 \\
C3hh & HM222432 \\
C3n & EU449106 \\
\hline
\end{tabular}


Appendix B: Supplementary data tables and figures 
Table B.1 Standard curve analysis for nested qPCR (North Bay colonies; chapter 2)

\begin{tabular}{|c|c|c|c|c|c|c|c|}
\hline Assay & $\begin{array}{l}\text { Template } \\
\text { sequence }\end{array}$ & $\begin{array}{l}\text { Concentration } \\
\left(\mathrm{ng} \boldsymbol{\mu l}^{-1}\right)\end{array}$ & $\begin{array}{l}\text { Concentration } \\
\left(\text { copies } \mu l^{-1}\right)\end{array}$ & Mean $C_{t}$ & Slope & Intercept & Efficiency \\
\hline $\mathrm{C} 100^{+}$ & C100 & $1 \times 10^{-3}$ & 212345 & 7.66 & -3.5255 & 26.472 & $96.08 \%$ \\
\hline \multirow[t]{8}{*}{ (TaqMan) } & & $1 \times 10^{-4}$ & 21234 & 11.12 & & & \\
\hline & & $1 \times 10^{-5}$ & 2123 & 14.91 & & & \\
\hline & & $1 \times 10^{-6}$ & 212 & 18.34 & & & \\
\hline & & $1 \times 10^{-7}$ & 21 & 21.68 & & & \\
\hline & C103 & $1 \times 10^{-3}$ & 212345 & - & & & \\
\hline & C109 & $2.5 \times 10^{-3}$ & 530862 & - & & & \\
\hline & C118 & $1 \times 10^{-3}$ & 212345 & - & & & \\
\hline & C3 & $1 \times 10^{-3}$ & 212345 & - & & & \\
\hline $\mathrm{C} 100^{-}$ & C109 & $2.5 \times 10^{-3}$ & 530862 & 6.14 & -3.5296 & 26.392 & $96.00 \%$ \\
\hline \multirow[t]{10}{*}{ (TaqMan) } & & $2.5 \times 10^{-4}$ & 53086 & 9.66 & & & \\
\hline & & $2.5 \times 10^{-5}$ & 5309 & 13.4 & & & \\
\hline & & $2.5 \times 10^{-6}$ & 531 & 16.8 & & & \\
\hline & & $2.5 \times 10^{-7}$ & 53 & 20.22 & & & \\
\hline & C103 & $1 \times 10^{-3}$ & 212345 & 8.33 & -3.5305 & 27.054 & $95.99 \%$ \\
\hline & & $1 \times 10^{-4}$ & 21234 & 11.67 & & & \\
\hline & & $1 \times 10^{-5}$ & 2123 & 15.45 & & & \\
\hline & & $1 \times 10^{-6}$ & 212 & 18.54 & & & \\
\hline & & $1 \times 10^{-7}$ & 21 & 22.51 & & & \\
\hline & $\mathrm{C} 100$ & $1 \times 10^{-3}$ & 212345 & - & & & \\
\hline \multirow[t]{5}{*}{ SYBR } & C100/C109 & $1 \times 10^{-3}$ & 212345 & 5.79 & -3.4024 & 23.775 & $98.37 \%$ \\
\hline & & $1 \times 10^{-4}$ & 21234 & 8.9 & & & \\
\hline & & $1 \times 10^{-5}$ & 2123 & 12.48 & & & \\
\hline & & $1 \times 10^{-6}$ & 212 & 15.73 & & & \\
\hline & & $1 \times 10^{-7}$ & 21 & 19.38 & & & \\
\hline
\end{tabular}

Mean cycling threshold $\left(\mathrm{C}_{\mathrm{t}}\right)$ values were calculated from triplicate reactions. Template solutions were plasmid-purified DNA of known ITS2 sequences. A C100/C109 mixture with $\mathrm{C}_{\mathrm{C} 100}$ : $\mathrm{C}_{\text {TOTAL }}=0.5$ was used for calibration of the SYBR assay. Dashes represent no-amplification reactions, and show an absence of cross-hybridization. $\mathrm{R}^{2}$ values exceeded 0.99 in all cases 
Table B.2 Standard curve analysis for nested qPCR (Ned's Beach colonies; chapter 2)

\begin{tabular}{|c|c|c|c|c|c|c|c|}
\hline $\begin{array}{l}\mathrm{qPCR} \\
\text { assay }\end{array}$ & $\begin{array}{l}\text { Template } \\
\text { sequence }\end{array}$ & $\begin{array}{l}\text { Concentration } \\
\left(\mathrm{ng} \mu^{-1}\right)\end{array}$ & $\begin{array}{l}\text { Concentration } \\
\left(\text { copies } \mu \mathbf{l}^{-1}\right)\end{array}$ & Mean $C_{t}$ & Slope & Intercept & Efficiency \\
\hline \multirow[t]{6}{*}{$\mathrm{C100}^{+}$} & $\mathrm{C} 100$ & $6.67 \times 10^{-4}$ & 142,271 & 7.66 & -3.5325 & 30.057 & $95.95 \%$ \\
\hline & & $1 \times 10^{-4}$ & 21,234 & 11.12 & & & \\
\hline & & $1 \times 10^{-5}$ & 2,123 & 14.91 & & & \\
\hline & & $1 \times 10^{-6}$ & 212 & 18.34 & & & \\
\hline & & $1 \times 10^{-7}$ & 21 & 21.68 & & & \\
\hline & C109 & $1 \times 10^{-3}$ & 212,344 & - & & & \\
\hline \multirow[t]{6}{*}{$\mathrm{C}^{100}{ }^{-}$} & C109 & $1 \times 10^{-3}$ & 212,344 & 6.14 & -3.553 & 29.866 & $95.59 \%$ \\
\hline & & $2.5 \times 10^{-4}$ & 53,086 & 9.66 & & & \\
\hline & & $2.5 \times 10^{-5}$ & 5,309 & 13.4 & & & \\
\hline & & $2.5 \times 10^{-6}$ & 531 & 16.8 & & & \\
\hline & & $2.5 \times 10^{-7}$ & 53 & 20.22 & & & \\
\hline & $\mathrm{C} 100$ & $6.67 \times 10^{-4}$ & 142,271 & - & & & \\
\hline
\end{tabular}

Mean cycling threshold $\left(\mathrm{C}_{\mathrm{t}}\right)$ values are calculated from triplicate reactions. Template solutions were plasmid-purified DNA of known ITS2 sequences (C100 or C109). Dashes represent no-amplification reactions, and show an absence of cross-hybridization. $\mathrm{R}^{2}$ values exceeded 0.99 in both cases. 
Table B.3 Assay validation for TaqMan nested qPCR (chapter 2)

\begin{tabular}{llllll}
\hline Mixture & $\begin{array}{l}\mathbf{C}_{\mathbf{C 1 0 0}}: \mathbf{C}_{\text {TOtal }} \\
(\mathbf{a c t u a l})\end{array}$ & Mean $\mathbf{C}_{\mathbf{t}}\left(\mathbf{C 1 0 0}^{+}\right)$ & Mean $\mathbf{C}_{\mathbf{t}}\left(\mathbf{C 1 0 0}^{-}\right)$ & $\begin{array}{l}\mathbf{C}_{\text {TOTAL }} \\
\text { predicted) }\end{array}$ & $\begin{array}{l}\mathbf{C}_{\mathbf{C 1 0 0}}: \mathbf{C}_{\text {TOTAL }} \\
(\mathbf{p r e d i c t e d})\end{array}$ \\
\hline $\mathbf{1}$ & 1 & 18.45 & - & 188 & 1 \\
$\mathbf{2}$ & 0.98 & 18.44 & 23.7 & 195 & 0.970 \\
$\mathbf{3}$ & 0.9 & 18.53 & 21.26 & 207 & 0.863 \\
$\mathbf{4}$ & 0.6 & 19.09 & 19.54 & 211 & 0.586 \\
$\mathbf{5}$ & 0.4 & 19.67 & 19.08 & 203 & 0.418 \\
$\mathbf{6}$ & 0.1 & 21.49 & 18.51 & 197 & 0.132 \\
$\mathbf{7}$ & 0.02 & 24.99 & 18.38 & 189 & 0.014 \\
$\mathbf{8}$ & 0 & - & 18.4 & 184 & 0 \\
\hline
\end{tabular}

Mixtures were generated from plasmid C100 and C109 template solutions diluted to approximately 200 ITS 2 copies $\mu 1^{-1}$. The assay predicted $\mathrm{C}_{\mathrm{C} 100}: \mathrm{C}_{\mathrm{TOTAL}}$ with a high degree of accuracy and precision (linear regression with constrained parameters; intercept $=0$ and slope $=1 ; \mathrm{R}^{2}=0.998$ ). Dashes represent no-amplification reactions, and show an absence of cross-hybridization.
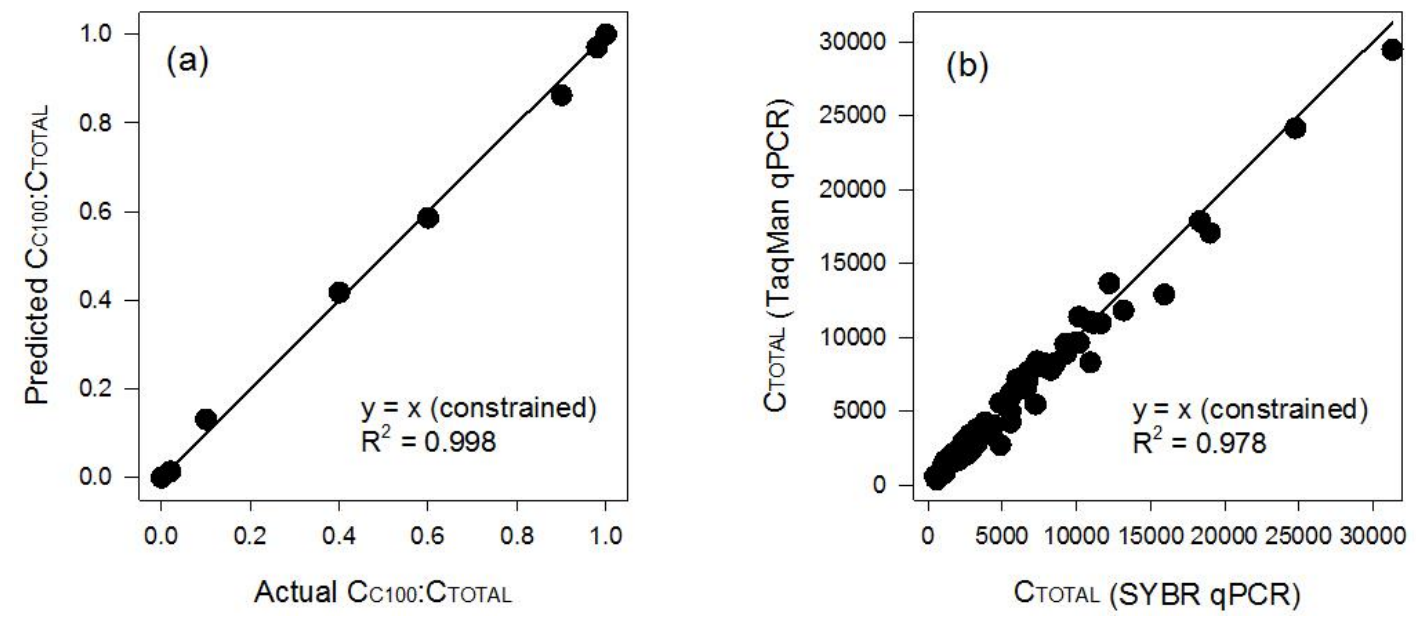

Figure B.1 Single-cell qPCR assay validation (chapter 2)

The TaqMan qPCR assay was validated by: (a) testing with known mixtures of clonal DNA; and (b) comparing copy-number estimates for each Symbiodinium cell (North Bay colonies) with those obtained from the independent SYBR qPCR assay. TaqMan and SYBR qPCR methods gave highly congruent results, implying that the number of ITS2 copies present within the genome that were not detected by either TaqMan probe was negligible. 
Table B.4 Mean $\mathrm{C}_{\mathrm{t}}$ values for individual Symbiodinium cells (colony a; chapter 2)

\begin{tabular}{|c|c|c|c|c|c|c|c|c|}
\hline Branch & C100 band & C109 band & Mean $\mathrm{C}_{\mathrm{t}}\left(\mathrm{C100}^{+}\right)$ & Mean $\mathrm{C}_{\mathrm{t}}\left(\mathrm{C100}^{-}\right)$ & Mean $C_{t}(S Y B R)$ & $\mathrm{C}_{\text {TOTAL }}$ (TaqMan) & $\mathrm{C}_{\text {TOTAL }}$ (SYBR) & $\mathrm{C}_{\mathrm{C} 100}: \mathrm{C}_{\text {TOTAL }}$ \\
\hline \multirow[t]{10}{*}{1} & $\mathrm{Y}$ & $\mathrm{Y}$ & 19.72 & 20.89 & 16.97 & 2364 & 1998 & 0.6945 \\
\hline & $\mathrm{Y}$ & $\mathrm{N}$ & 20.45 & 24.89 & 17.89 & 1077 & 1072 & 0.9505 \\
\hline & $\mathrm{N}$ & $\mathrm{Y}$ & - & 21.22 & 19.26 & 584 & 426 & 0 \\
\hline & $\mathrm{Y}$ & $\mathrm{Y}$ & 19.32 & 20.73 & 16.65 & 2944 & 2479 & 0.7278 \\
\hline & $\mathrm{Y}$ & $\mathrm{Y}$ & 19.75 & 20.69 & 16.84 & 2435 & 2178 & 0.6616 \\
\hline & $\mathrm{Y}$ & $\mathrm{Y}$ & 21.43 & 21.69 & 18.12 & 970 & 917 & 0.5566 \\
\hline & $\mathrm{Y}$ & $\mathrm{Y}$ & 20.52 & 21.13 & 17.35 & 1598 & 1542 & 0.6111 \\
\hline & $\mathrm{Y}$ & $\mathrm{Y}$ & 21 & 22.25 & 18 & 1010 & 995 & 0.7049 \\
\hline & $\mathrm{Y}$ & $\mathrm{N}$ & 19.37 & 21.24 & 16.65 & 2644 & 2482 & 0.7822 \\
\hline & $\mathrm{Y}$ & $\mathrm{Y}$ & 20.69 & 22.4 & 17.88 & 1145 & 1083 & 0.7639 \\
\hline \multirow[t]{10}{*}{2} & $\mathrm{Y}$ & $\mathrm{Y}$ & 20.24 & 21.54 & 17.79 & 1645 & 1150 & 0.7126 \\
\hline & $\mathrm{Y}$ & $\mathrm{Y}$ & 20.95 & 20.51 & 17.21 & 1662 & 1701 & 0.4424 \\
\hline & $\mathrm{Y}$ & $\mathrm{Y}$ & 22.34 & 23.25 & 18.97 & 453 & 516 & 0.6561 \\
\hline & $\mathrm{Y}$ & $\mathrm{Y}$ & 21.19 & 23.05 & 17.86 & 809 & 1097 & 0.7805 \\
\hline & $\mathrm{Y}$ & $\mathrm{N}$ & 19.31 & 21.58 & 16.82 & 2606 & 2215 & 0.8233 \\
\hline & $\mathrm{Y}$ & $\mathrm{Y}$ & 19.52 & 20.56 & 16.26 & 2767 & 3226 & 0.6758 \\
\hline & $\mathrm{Y}$ & $\mathrm{Y}$ & 19.09 & 20.49 & 16.46 & 3426 & 2825 & 0.7254 \\
\hline & $\mathrm{Y}$ & $\mathrm{Y}$ & 18.82 & 20.6 & 15.93 & 3828 & 4031 & 0.7721 \\
\hline & $\mathrm{Y}$ & $\mathrm{Y}$ & 21.69 & 23.12 & 18.36 & 625 & 782 & 0.7288 \\
\hline & $\mathrm{Y}$ & $\mathrm{N}$ & 19.89 & 22.1 & 17.16 & 1804 & 1758 & 0.8179 \\
\hline \multirow[t]{10}{*}{3} & $\mathrm{Y}$ & $\mathrm{Y}$ & 17.05 & 18.48 & 13.91 & 12896 & 15919 & 0.7292 \\
\hline & $\mathrm{Y}$ & $\mathrm{Y}$ & 19.27 & 20.42 & 16.4 & 3200 & 2947 & 0.692 \\
\hline & $\mathrm{Y}$ & $\mathrm{Y}$ & 22.08 & 22.78 & 18.5 & 565 & 710 & 0.625 \\
\hline & $\mathrm{Y}$ & $\mathrm{N}$ & 18.97 & 20.5 & 16.26 & 3626 & 3224 & 0.7418 \\
\hline & $\mathrm{Y}$ & $\mathrm{Y}$ & 19.3 & 21.02 & 16.5 & 2830 & 2755 & 0.7646 \\
\hline & $\mathrm{Y}$ & $\mathrm{N}$ & 19.03 & 21.09 & 16.36 & 3210 & 3033 & 0.8025 \\
\hline & $\mathrm{Y}$ & $\mathrm{Y}$ & 20.66 & 21.15 & 17.57 & 1504 & 1331 & 0.5924 \\
\hline & $\mathrm{Y}$ & $\mathrm{Y}$ & 20.43 & 21.52 & 17.27 & 1515 & 1627 & 0.6826 \\
\hline & $\mathrm{Y}$ & $\mathrm{Y}$ & 19.27 & 19.49 & 15.8 & 4004 & 4416 & 0.55 \\
\hline & $\mathrm{Y}$ & $\mathrm{Y}$ & 19.53 & 20.26 & 16.73 & 2948 & 2360 & 0.6302 \\
\hline
\end{tabular}

C100- and C109-diagnostic DGGE bands are scored as present or absent (Y or N). Dashes represent no-amplification reactions 
Table B.5 Mean $\mathrm{C}_{\mathrm{t}}$ values for individual Symbiodinium cells (colony b; chapter 2)

\begin{tabular}{|c|c|c|c|c|c|c|c|c|}
\hline Branch & C100 band & C109 band & Mean $\mathrm{C}_{\mathrm{t}}\left(\mathrm{C100}^{+}\right)$ & Mean $\mathrm{C}_{\mathrm{t}}\left(\mathrm{C100}^{-}\right)$ & Mean $C_{t}(S Y B R)$ & $\mathrm{C}_{\text {TOTAL }}$ (TaqMan) & $\mathrm{C}_{\text {TOTAL }}$ (SYBR) & $\mathrm{C}_{\mathrm{C} 100}: \mathrm{C}_{\text {TOTAL }}$ \\
\hline \multirow[t]{10}{*}{1} & $\mathrm{Y}$ & $\mathrm{Y}$ & 20.14 & 21.99 & 17.3 & 1607 & 1603 & 0.7798 \\
\hline & $\mathrm{Y}$ & $\mathrm{Y}$ & 18.72 & 21.25 & 16.17 & 3736 & 3446 & 0.8466 \\
\hline & $\mathrm{Y}$ & $\mathrm{N}$ & 19.51 & 21.26 & 16.45 & 2459 & 2852 & 0.7682 \\
\hline & $\mathrm{Y}$ & $\mathrm{N}$ & 20.13 & 22.74 & 17.35 & 1475 & 1550 & 0.8532 \\
\hline & $\mathrm{Y}$ & $\mathrm{Y}$ & 19.99 & 21 & 16.87 & 2057 & 2138 & 0.672 \\
\hline & $\mathrm{Y}$ & $\mathrm{Y}$ & 20.01 & 20.87 & 16.54 & 2094 & 2667 & 0.6499 \\
\hline & $\mathrm{Y}$ & $\mathrm{Y}$ & 20.38 & 21.2 & 17.27 & 1663 & 1638 & 0.6434 \\
\hline & $\mathrm{Y}$ & $\mathrm{N}$ & 19.43 & 21.45 & 16.57 & 2489 & 2626 & 0.7977 \\
\hline & $\mathrm{Y}$ & $\mathrm{Y}$ & 18.57 & 20.84 & 16.02 & 4245 & 3812 & 0.8232 \\
\hline & $\mathrm{Y}$ & $\mathrm{N}$ & 19.78 & 22.72 & 17.31 & 1800 & 1588 & 0.878 \\
\hline \multirow[t]{10}{*}{2} & $\mathrm{~N}$ & $\mathrm{Y}$ & - & 21.89 & 18.81 & 377 & 577 & 0 \\
\hline & $\mathrm{Y}$ & $\mathrm{N}$ & 19.18 & 21.94 & 15.66 & 2708 & 4864 & 0.8653 \\
\hline & $\mathrm{Y}$ & $\mathrm{Y}$ & 19.7 & 21.11 & 16.43 & 2298 & 2884 & 0.7276 \\
\hline & $\mathrm{N}$ & $\mathrm{Y}$ & 25.18 & 20.74 & 18.32 & 848 & 801 & 0.0549 \\
\hline & $\mathrm{Y}$ & $\mathrm{N}$ & 18.13 & 22.15 & 15.46 & 4974 & 5542 & 0.9361 \\
\hline & $\mathrm{Y}$ & $\mathrm{N}$ & 19.55 & 22.19 & 16.98 & 2149 & 1987 & 0.8552 \\
\hline & $\mathrm{Y}$ & $\mathrm{N}$ & 20.11 & 23.16 & 17.5 & 1439 & 1401 & 0.8853 \\
\hline & $\mathrm{Y}$ & $\mathrm{Y}$ & 18.2 & 19.22 & 15.38 & 6597 & 5882 & 0.6727 \\
\hline & $\mathrm{Y}$ & $\mathrm{N}$ & 20.35 & 22.96 & 17.78 & 1277 & 1160 & 0.8528 \\
\hline & $\mathrm{Y}$ & $\mathrm{N}$ & 19.53 & 21.74 & 16.96 & 2282 & 2017 & 0.8178 \\
\hline \multirow[t]{10}{*}{3} & $\mathrm{Y}$ & $\mathrm{Y}$ & 20.52 & 21.88 & 17.75 & 1354 & 1178 & 0.7203 \\
\hline & $\mathrm{Y}$ & $\mathrm{N}$ & 17.5 & 20.02 & 14.46 & 8302 & 10922 & 0.8462 \\
\hline & $\mathrm{Y}$ & $\mathrm{Y}$ & 20.52 & 20.93 & 16.93 & 1683 & 2052 & 0.5812 \\
\hline & $\mathrm{Y}$ & $\mathrm{Y}$ & 19.29 & 20.1 & 15.87 & 3393 & 4212 & 0.6438 \\
\hline & $\mathrm{Y}$ & $\mathrm{N}$ & 20.54 & 26.28 & 17.9 & 985 & 1066 & 0.9781 \\
\hline & $\mathrm{Y}$ & $\mathrm{N}$ & 19.55 & 22.53 & 17.3 & 2088 & 1602 & 0.8808 \\
\hline & $\mathrm{Y}$ & $\mathrm{N}$ & 19.33 & 21.03 & 16.51 & 2783 & 2727 & 0.7626 \\
\hline & $\mathrm{Y}$ & $\mathrm{Y}$ & 18.92 & 20.34 & 16.24 & 3803 & 3289 & 0.7279 \\
\hline & $\mathrm{Y}$ & $\mathrm{N}$ & 17.97 & 20.34 & 15.47 & 6187 & 5535 & 0.832 \\
\hline & $\mathrm{Y}$ & $\mathrm{N}$ & 17.61 & 21.04 & 15.35 & 7168 & 5981 & 0.9082 \\
\hline
\end{tabular}

C100- and C109-diagnostic DGGE bands are scored as present or absent (Y or N). Dashes represent no-amplification reactions 
Table B.6 Mean $\mathrm{C}_{\mathrm{t}}$ values for individual Symbiodinium cells (colony c; chapter 2)

\begin{tabular}{|c|c|c|c|c|c|c|c|c|}
\hline Branch & C100 band & C109 band & Mean $\mathrm{C}_{\mathrm{t}}\left(\mathrm{C100}^{+}\right)$ & Mean $C_{t}\left(\mathrm{C100}^{-}\right)$ & Mean $C_{t}($ SYBR $)$ & $\mathrm{C}_{\text {TOTAL }}$ (TaqMan) & $\mathbf{C}_{\text {TOTAL }}(\mathrm{SYBR})$ & $\mathrm{C}_{\mathrm{C} 100}: \mathrm{C}_{\mathrm{TOTAL}}$ \\
\hline \multirow[t]{10}{*}{1} & $\mathrm{Y}$ & $\mathrm{N}$ & 16.87 & 20.74 & 14.57 & 11391 & 10152 & 0.9299 \\
\hline & $\mathrm{Y}$ & $\mathrm{N}$ & 17.88 & 23.9 & 15.67 & 5557 & 4834 & 0.9817 \\
\hline & $\mathrm{Y}$ & $\mathrm{N}$ & 17.36 & 23.9 & 14.87 & 7778 & 8259 & 0.9869 \\
\hline & $\mathrm{Y}$ & $\mathrm{N}$ & 16.54 & 21.34 & 14.3 & 13651 & 12210 & 0.9603 \\
\hline & $\mathrm{Y}$ & $\mathrm{N}$ & 17.24 & 21.22 & 14.7 & 8895 & 9301 & 0.9345 \\
\hline & $\mathrm{Y}$ & $\mathrm{N}$ & 17.6 & 21.65 & 15.19 & 7026 & 6683 & 0.9372 \\
\hline & $\mathrm{Y}$ & $\mathrm{N}$ & 15.67 & 20.37 & 13.25 & 24149 & 24764 & 0.958 \\
\hline & $\mathrm{Y}$ & $\mathrm{N}$ & 16.18 & 21.75 & 13.64 & 17066 & 18997 & 0.9757 \\
\hline & $\mathrm{Y}$ & $\mathrm{N}$ & 17.6 & 21.96 & 15.23 & 6915 & 6473 & 0.9478 \\
\hline & $\mathrm{Y}$ & $\mathrm{N}$ & 17.77 & 22.99 & 15.44 & 6048 & 5620 & 0.9695 \\
\hline \multirow[t]{10}{*}{2} & $\mathrm{Y}$ & $\mathrm{N}$ & 17.93 & 23.1 & 15.07 & 5460 & 7233 & 0.9686 \\
\hline & $\mathrm{Y}$ & $\mathrm{N}$ & 16.14 & 20.73 & 13.7 & 17854 & 18335 & 0.955 \\
\hline & $\mathrm{Y}$ & $\mathrm{N}$ & 17.12 & 21.02 & 14.57 & 9625 & 10166 & 0.931 \\
\hline & $\mathrm{Y}$ & $\mathrm{N}$ & 15.4 & 19.49 & 12.91 & 29461 & 31310 & 0.9388 \\
\hline & $\mathrm{Y}$ & $\mathrm{N}$ & 17.16 & 20.78 & 14.71 & 9559 & 9239 & 0.9184 \\
\hline & $\mathrm{Y}$ & $\mathrm{N}$ & 17.35 & 22.28 & 14.95 & 8019 & 7854 & 0.9635 \\
\hline & $\mathrm{Y}$ & $\mathrm{N}$ & 18.47 & 21.43 & 15.46 & 4231 & 5547 & 0.8799 \\
\hline & $\mathrm{Y}$ & $\mathrm{Y}$ & 21.24 & 20.95 & 18.07 & 1305 & 951 & 0.4659 \\
\hline & $\mathrm{Y}$ & $\mathrm{N}$ & 17.45 & 21.7 & 15.18 & 7667 & 6733 & 0.9445 \\
\hline & $\mathrm{Y}$ & $\mathrm{N}$ & 17.31 & 23.75 & 15.01 & 8034 & 7554 & 0.986 \\
\hline \multirow[t]{10}{*}{3} & $\mathrm{Y}$ & $\mathrm{N}$ & 16.84 & 20.33 & 14.19 & 11815 & 13153 & 0.9116 \\
\hline & $\mathrm{Y}$ & $\mathrm{N}$ & 16.89 & 21.16 & 14.46 & 11056 & 10918 & 0.945 \\
\hline & $\mathrm{Y}$ & $\mathrm{N}$ & 17.35 & 21.32 & 14.98 & 8266 & 7697 & 0.9338 \\
\hline & $\mathrm{Y}$ & $\mathrm{Y}$ & 19.52 & 20.56 & 16.7 & 2776 & 2396 & 0.6758 \\
\hline & $\mathrm{Y}$ & $\mathrm{N}$ & 17.15 & 20.7 & 14.59 & 9661 & 10009 & 0.9149 \\
\hline & $\mathrm{Y}$ & $\mathrm{N}$ & 16.87 & 22.02 & 14.37 & 10947 & 11643 & 0.9684 \\
\hline & $\mathrm{Y}$ & $\mathrm{N}$ & 17.38 & 20.77 & 15.05 & 8387 & 7339 & 0.9066 \\
\hline & $\mathrm{Y}$ & $\mathrm{N}$ & 16.88 & 21.62 & 14.43 & 10956 & 11144 & 0.959 \\
\hline & $\mathrm{Y}$ & $\mathrm{N}$ & 17.65 & 23.72 & 15.21 & 6491 & 6571 & 0.9824 \\
\hline & $\mathrm{Y}$ & $\mathrm{N}$ & 17.32 & 21.89 & 14.82 & 8276 & 8585 & 0.9545 \\
\hline
\end{tabular}

C100- and C109-diagnostic DGGE bands are scored as present or absent (Y or N). Dashes represent no-amplification reactions 
Table B.7 Mean $\mathrm{C}_{\mathrm{t}}$ values for individual Symbiodinium cells (colony d; chapter 2)

\begin{tabular}{|c|c|c|c|c|c|c|c|}
\hline Branch & C100 band & C109 band & Mean $\mathrm{C}_{\mathrm{t}}\left(\mathrm{C100}^{+}\right)$ & Mean $C_{t}\left(\mathrm{C100}^{-}\right)$ & $\mathrm{C}_{\mathrm{C} 100}$ & $\mathbf{C}_{\text {TOTAL }}$ & $\mathbf{C}_{\text {C100: }}: \mathbf{C}_{\text {TOTAL }}$ \\
\hline \multirow[t]{10}{*}{1} & $\mathrm{~N}$ & $\mathrm{Y}$ & 27.01 & 23.92 & 146 & 1092 & 0.1339 \\
\hline & $\mathrm{Y}$ & $\mathrm{Y}$ & 23.26 & 23.75 & 1685 & 2741 & 0.6147 \\
\hline & $\mathrm{Y}$ & $\mathrm{Y}$ & 23.63 & 23.56 & 1324 & 2515 & 0.5264 \\
\hline & $\mathrm{Y}$ & $\mathrm{Y}$ & 24.01 & 24.49 & 1033 & 1685 & 0.6132 \\
\hline & $\mathrm{Y}$ & $\mathrm{Y}$ & 24.64 & 25 & 683 & 1153 & 0.5925 \\
\hline & $\mathrm{Y}$ & Y & 25.16 & 24.78 & 487 & 1029 & 0.4732 \\
\hline & $\mathrm{Y}$ & $\mathrm{Y}$ & 22.58 & 23.16 & 2616 & 4164 & 0.6282 \\
\hline & $\mathrm{Y}$ & Y & 25.02 & 25.36 & 533 & 905 & 0.589 \\
\hline & $\mathrm{Y}$ & Y & 22.28 & 23.6 & 3192 & 4356 & 0.7327 \\
\hline & $\mathrm{Y}$ & Y & 23.65 & 23.88 & 1307 & 2278 & 0.5737 \\
\hline \multirow[t]{10}{*}{2} & $\mathrm{Y}$ & $\mathrm{N}$ & 23.25 & 30.62 & 1690 & 1703 & 0.9928 \\
\hline & $\mathrm{Y}$ & Y & 26.5 & 27.41 & 203 & 301 & 0.6741 \\
\hline & $\mathrm{Y}$ & Y & 22.76 & 22.58 & 2334 & 4589 & 0.5086 \\
\hline & $\mathrm{Y}$ & $\mathrm{N}$ & 23.13 & 25.44 & 1828 & 2180 & 0.8385 \\
\hline & $\mathrm{Y}$ & $\mathrm{Y}$ & 21.98 & 22.47 & 3868 & 6282 & 0.6158 \\
\hline & $\mathrm{Y}$ & Y & 20.69 & 20.98 & 8968 & 15307 & 0.5859 \\
\hline & $\mathrm{Y}$ & Y & 28.19 & 28.94 & 68 & 104 & 0.6495 \\
\hline & $\mathrm{Y}$ & Y & 23.4 & 23.88 & 1533 & 2501 & 0.613 \\
\hline & $\mathrm{Y}$ & Y & 27.72 & 28.52 & 92 & 140 & 0.6565 \\
\hline & $\mathrm{Y}$ & Y & 22.84 & 23.02 & 2208 & 3898 & 0.5665 \\
\hline \multirow[t]{10}{*}{3} & $\mathrm{Y}$ & $\mathrm{Y}$ & 26.96 & 27.41 & 151 & 250 & 0.6052 \\
\hline & $\mathrm{Y}$ & $\mathrm{N}$ & 21.19 & 23.37 & 6474 & 7821 & 0.8278 \\
\hline & $\mathrm{Y}$ & Y & 24.32 & 24.89 & 844 & 1349 & 0.6259 \\
\hline & Y & Y & 21.67 & 22.19 & 4750 & 7653 & 0.6207 \\
\hline & $\mathrm{Y}$ & Y & 25.2 & 25.73 & 476 & 769 & 0.6191 \\
\hline & $\mathrm{Y}$ & $\mathrm{Y}$ & 26.77 & 27.69 & 171 & 253 & 0.6753 \\
\hline & $\mathrm{Y}$ & $\mathrm{Y}$ & 27.33 & 27.98 & 118 & 186 & 0.6354 \\
\hline & $\mathrm{Y}$ & $\mathrm{N}$ & 19.94 & 23.11 & 14622 & 16221 & 0.9014 \\
\hline & $\mathrm{N}$ & Y & 32.31 & 24.13 & 5 & 828 & 0.0056 \\
\hline & Y & Y & 21.27 & 21.6 & 6145 & 10400 & 0.5908 \\
\hline
\end{tabular}

C100- and C109-diagnostic DGGE bands are scored as present or absent (Y or N). Dashes represent no-amplification reactions 
Table B.8 Mean $\mathrm{C}_{\mathrm{t}}$ values for individual Symbiodinium cells (colony e; chapter 2)

\begin{tabular}{|c|c|c|c|c|c|c|c|}
\hline Branch & C100 band & C109 band & Mean $\mathrm{C}_{\mathrm{t}}\left(\mathrm{C100}^{+}\right)$ & Mean $\mathrm{C}_{\mathrm{t}}\left(\mathrm{C100}^{-}\right)$ & $\mathrm{C}_{\mathrm{C} 100}$ & $\mathbf{C}_{\text {TOTAL }}$ & $\mathrm{C}_{\mathrm{C} 100}: \mathrm{C}_{\mathrm{TOTAL}}$ \\
\hline \multirow[t]{10}{*}{1} & $\bar{Y}$ & $\mathrm{~N}$ & 20.81 & 28.04 & 8320 & 8386 & 0.9922 \\
\hline & Y & $\mathrm{N}$ & 23.74 & 30.54 & 1232 & 1245 & 0.9896 \\
\hline & $\mathrm{Y}$ & $\mathrm{N}$ & 23.73 & 25.9 & 1236 & 1498 & 0.825 \\
\hline & $\mathrm{Y}$ & $\mathrm{Y}$ & 23.6 & 25 & 1350 & 1820 & 0.7418 \\
\hline & $\mathrm{Y}$ & $\mathrm{Y}$ & 23.34 & 25.19 & 1599 & 2013 & 0.7943 \\
\hline & Y & $\mathrm{N}$ & 21.36 & 24.31 & 5814 & 6548 & 0.8878 \\
\hline & $\mathrm{Y}$ & $\mathrm{N}$ & 24.62 & 27.33 & 692 & 796 & 0.8696 \\
\hline & $\mathrm{Y}$ & $\mathrm{N}$ & 18.78 & 25.26 & 31145 & 31541 & 0.9875 \\
\hline & $\mathrm{Y}$ & $\mathrm{N}$ & 19.48 & 23.86 & 19799 & 20783 & 0.9527 \\
\hline & Y & Y & 21.75 & 22.94 & 4494 & 6274 & 0.7163 \\
\hline \multirow[t]{10}{*}{2} & $\mathrm{Y}$ & $\mathrm{Y}$ & 20.66 & 22.41 & 9175 & 11692 & 0.7847 \\
\hline & $\mathrm{Y}$ & $\mathrm{Y}$ & 21.5 & 22.69 & 5307 & 7399 & 0.7172 \\
\hline & $\mathrm{Y}$ & $\mathrm{N}$ & 21.45 & 26 & 5482 & 5727 & 0.9572 \\
\hline & Y & $\mathrm{N}$ & 20.24 & 26.01 & 12025 & 12268 & 0.9802 \\
\hline & $\mathrm{Y}$ & $\mathrm{Y}$ & 23.67 & 24.45 & 1290 & 1959 & 0.6585 \\
\hline & $\mathrm{Y}$ & $\mathrm{N}$ & 21.99 & 24.35 & 3843 & 4557 & 0.8434 \\
\hline & $\mathrm{Y}$ & $\mathrm{N}$ & 22.37 & 29.11 & 3010 & 3042 & 0.9893 \\
\hline & $\mathrm{Y}$ & $\mathrm{N}$ & 22.58 & 29.74 & 2625 & 2646 & 0.9918 \\
\hline & Y & $\mathrm{N}$ & 20.32 & 27.56 & 11414 & 11503 & 0.9923 \\
\hline & $\mathrm{Y}$ & $\mathrm{N}$ & 27.74 & 29.88 & 91 & 111 & 0.8204 \\
\hline \multirow[t]{10}{*}{3} & $\mathrm{Y}$ & $\mathrm{N}$ & 23.64 & 30.62 & 1315 & 1328 & 0.9908 \\
\hline & Y & $\mathrm{N}$ & 24.39 & 31.05 & 804 & 813 & 0.9885 \\
\hline & $\mathrm{Y}$ & $\mathrm{N}$ & 24.83 & 31.47 & 604 & 611 & 0.9884 \\
\hline & $\mathrm{Y}$ & $\mathrm{N}$ & 19.83 & 23.11 & 15709 & 17308 & 0.9076 \\
\hline & $\mathrm{Y}$ & $\mathrm{N}$ & 21.34 & 26.63 & 5890 & 6053 & 0.973 \\
\hline & Y & Y & 21.05 & 23.12 & 7092 & 8681 & 0.817 \\
\hline & $\mathrm{Y}$ & $\mathrm{Y}$ & 26.72 & 27.19 & 176 & 290 & 0.6077 \\
\hline & Y & $\mathrm{N}$ & 21.76 & 26.76 & 4479 & 4629 & 0.9676 \\
\hline & $\mathrm{Y}$ & $\mathrm{N}$ & 23.07 & 27.5 & 1907 & 2000 & 0.9535 \\
\hline & $\mathrm{Y}$ & $\mathrm{N}$ & 22.19 & 24.91 & 3373 & 3870 & 0.8717 \\
\hline
\end{tabular}

C100- and C109-diagnostic DGGE bands are scored as present or absent (Y or N). Dashes represent no-amplification reactions 
Table B.9 Mean $\mathrm{C}_{\mathrm{t}}$ values for individual Symbiodinium cells (colony $\mathrm{f}$; chapter 2 )

\begin{tabular}{|c|c|c|c|c|c|c|c|}
\hline Branch & C100 band & C109 band & Mean $\mathrm{C}_{\mathrm{t}}\left(\mathrm{C100}^{+}\right)$ & Mean $C_{t}\left(\mathrm{C100}^{-}\right)$ & $\mathrm{C}_{\mathrm{C} 100}$ & $\mathbf{C}_{\text {TOTAL }}$ & $\mathbf{C}_{\text {C100 }}: \mathbf{C}_{\text {TOTAL }}$ \\
\hline \multirow[t]{10}{*}{1} & $\mathrm{Y}$ & $\mathrm{N}$ & 27.91 & 30.16 & 81 & 98 & 0.8306 \\
\hline & $\mathrm{Y}$ & $\mathrm{Y}$ & 26.25 & 28.12 & 240 & 302 & 0.7947 \\
\hline & $\mathrm{Y}$ & $\mathrm{N}$ & 24.37 & 32.07 & 815 & 819 & 0.9941 \\
\hline & $\mathrm{Y}$ & $\mathrm{Y}$ & 22.59 & 23.17 & 2608 & 4146 & 0.629 \\
\hline & $\mathrm{Y}$ & $\mathrm{Y}$ & 22.83 & 22.84 & 2230 & 4135 & 0.5393 \\
\hline & $\mathrm{Y}$ & $\mathrm{Y}$ & 22.68 & 23.59 & 2459 & 3627 & 0.678 \\
\hline & $\mathrm{Y}$ & $\mathrm{Y}$ & 22.44 & 22.12 & 2866 & 5904 & 0.4855 \\
\hline & $\mathrm{Y}$ & $\mathrm{N}$ & 21.9 & 29.65 & 4075 & 4098 & 0.9944 \\
\hline & $\mathrm{Y}$ & $\mathrm{N}$ & 24.68 & 32.27 & 666 & 670 & 0.9937 \\
\hline & $\mathrm{Y}$ & $\mathrm{Y}$ & 28.9 & 30.18 & 43 & 59 & 0.7227 \\
\hline \multirow[t]{10}{*}{2} & $\mathrm{Y}$ & $\mathrm{N}$ & 21.77 & 26.08 & 4450 & 4684 & 0.9502 \\
\hline & $\mathrm{N}$ & $\mathrm{Y}$ & 32.94 & 23.46 & 3 & 1274 & 0.0024 \\
\hline & $\mathrm{Y}$ & $\mathrm{N}$ & 23.09 & 30.7 & 1882 & 1894 & 0.9938 \\
\hline & $\mathrm{Y}$ & $\mathrm{Y}$ & 20.99 & 21.69 & 7375 & 11389 & 0.6476 \\
\hline & Y & Y & 25.18 & 25.07 & 480 & 929 & 0.5169 \\
\hline & $\mathrm{Y}$ & $\mathrm{Y}$ & 28.12 & 29 & 71 & 106 & 0.6678 \\
\hline & $\mathrm{Y}$ & $\mathrm{Y}$ & 26.99 & 27.55 & 148 & 238 & 0.622 \\
\hline & $\mathrm{Y}$ & $\mathrm{N}$ & 24.51 & 28 & 746 & 813 & 0.9173 \\
\hline & Y & $\mathrm{N}$ & 23.56 & 30.55 & 1386 & 1399 & 0.9908 \\
\hline & Y & $\mathrm{Y}$ & 22.92 & 23.87 & 2096 & 3073 & 0.682 \\
\hline \multirow[t]{10}{*}{3} & $\mathrm{Y}$ & $\mathrm{Y}$ & 21.99 & 22.88 & 3843 & 5693 & 0.675 \\
\hline & Y & $\mathrm{N}$ & 22.93 & 26.7 & 2082 & 2239 & 0.9303 \\
\hline & Y & $\mathrm{N}$ & 19.57 & 21.65 & 18671 & 22777 & 0.8197 \\
\hline & $\mathrm{Y}$ & $\mathrm{Y}$ & 22.88 & 24.71 & 2151 & 2717 & 0.792 \\
\hline & $\mathrm{Y}$ & $\mathrm{Y}$ & 24.29 & 25.36 & 861 & 1233 & 0.6982 \\
\hline & Y & Y & 25.27 & 25.73 & 455 & 746 & 0.609 \\
\hline & Y & $\mathrm{N}$ & 23.4 & 30.44 & 1538 & 1552 & 0.9911 \\
\hline & $\mathrm{Y}$ & $\mathrm{Y}$ & 23.84 & 23.76 & 1154 & 2201 & 0.5246 \\
\hline & $\mathrm{Y}$ & $\mathrm{N}$ & 22.78 & 26.21 & 2304 & 2518 & 0.9151 \\
\hline & Y & $\mathrm{Y}$ & 22.82 & 23.23 & 2237 & 3717 & 0.6019 \\
\hline
\end{tabular}

C100- and C109-diagnostic DGGE bands are scored as present or absent (Y or N). Dashes represent no-amplification reactions 
Table B.10 Standard curve parameter estimation for bulk-cell qPCR analysis (chapter 3)

\begin{tabular}{|c|c|c|c|c|c|c|c|}
\hline $\begin{array}{l}\text { qPCR } \\
\text { assay }\end{array}$ & $\begin{array}{l}\text { Template } \\
\text { sequence }\end{array}$ & $\begin{array}{l}\text { Concentration } \\
\left(n g \mu \mathrm{I}^{-1}\right)\end{array}$ & $\begin{array}{l}\text { Concentration } \\
\left(\text { copies } \mu \mathrm{l}^{-1}\right)\end{array}$ & Mean $C_{t}$ & Slope & Intercept & Efficiency \\
\hline \multirow[t]{7}{*}{$\mathrm{C}^{100}{ }^{+}$} & $\mathrm{C} 100$ & $1 \times 10^{-2}$ & $2,123,448$ & 15.41 & -3.379 & 36.873 & $98.84 \%$ \\
\hline & & $1 \times 10^{-3}$ & 212,345 & 18.87 & & & \\
\hline & & $1 \times 10^{-4}$ & 21,234 & 22.16 & & & \\
\hline & & $1 \times 10^{-5}$ & 2,123 & 25.91 & & & \\
\hline & & $1 \times 10^{-6}$ & 212 & 29.2 & & & \\
\hline & & $1 \times 10^{-7}$ & 21 & 32.11 & & & \\
\hline & C109 & $2.5 \times 10^{-2}$ & $5,308,621$ & - & & & \\
\hline \multirow[t]{7}{*}{$\mathrm{C100}^{-}$} & C109 & $2.5 \times 10^{-2}$ & $5,308,621$ & 14.59 & -3.373 & 37.119 & $98.96 \%$ \\
\hline & & $2.5 \times 10^{-3}$ & 53,0862 & 17.83 & & & \\
\hline & & $2.5 \times 10^{-4}$ & 53,086 & 20.68 & & & \\
\hline & & $2.5 \times 10^{-5}$ & 5,309 & 24.94 & & & \\
\hline & & $2.5 \times 10^{-6}$ & 531 & 27.82 & & & \\
\hline & & $2.5 \times 10^{-7}$ & 53 & 31.35 & & & \\
\hline & C100 & $1 \times 10^{-2}$ & $2,123,448$ & - & & & \\
\hline
\end{tabular}

Mean cycling threshold $\left(\mathrm{C}_{\mathrm{t}}\right)$ values are calculated from triplicate reactions. Template solutions were plasmid-purified DNA of known ITS2 sequences (C100 or C109). Dashes represent no-amplification reactions, and show an absence of cross-hybridization. $\mathrm{R}^{2}$ values exceeded 0.99 in both cases. 
Table B.11 Nested analysis of spatial variance in $S_{\mathrm{H}}: S_{\mathrm{T}}$ (chapter 3 )

\begin{tabular}{|c|c|c|c|c|c|c|c|c|c|c|c|}
\hline Site & ID & Branch & C100 band & C109 band & Mean $\mathrm{C}_{\mathrm{t}}\left(\mathrm{C100}^{+}\right)$ & Mean $C_{t}\left(\mathrm{C100}^{-}\right)$ & $\mathrm{C}_{\mathrm{C} 100}$ & $\mathrm{C}_{\mathrm{C} 100}{ }^{-}$ & $\mathbf{C}_{\text {TOtaL }}$ & $\mathrm{C}_{100}: \mathrm{C}_{\text {TOTAL }}$ & $S_{\mathrm{H}}: S_{\mathrm{T}}$ \\
\hline Sylph's & 1 & 1 & $\mathrm{Y}$ & $\mathrm{N}$ & 21.62 & 25.6 & 32698 & 2609 & 35306 & 0.926 & 0.154 \\
\hline \multirow[t]{14}{*}{ Hole } & & 2 & $\mathrm{Y}$ & $\mathrm{N}$ & 21.14 & 25 & 45173 & 3915 & 49088 & 0.92 & 0.172 \\
\hline & & 3 & $\mathrm{Y}$ & $\mathrm{N}$ & 21.35 & 25.76 & 39250 & 2338 & 41588 & 0.944 & 0.1 \\
\hline & 2 & 1 & $\mathrm{Y}$ & $\mathrm{Y}$ & 25.63 & 27.89 & 2128 & 545 & 2673 & 0.796 & 0.49 \\
\hline & & 2 & $\mathrm{Y}$ & $\mathrm{Y}$ & 20.25 & 22.52 & 82843 & 21228 & 104071 & 0.796 & 0.49 \\
\hline & & 3 & $\mathrm{Y}$ & $\mathrm{Y}$ & 24.44 & 26.85 & 4772 & 1111 & 5883 & 0.811 & 0.457 \\
\hline & 3 & 1 & $\mathrm{Y}$ & $\mathrm{Y}$ & 27.33 & 29.28 & 666 & 210 & 876 & 0.76 & 0.562 \\
\hline & & 2 & $\mathrm{Y}$ & $\mathrm{Y}$ & 25.36 & 27.41 & 2561 & 755 & 3317 & 0.772 & 0.539 \\
\hline & & 3 & $\mathrm{Y}$ & $\mathrm{N}$ & 22.7 & 24.7 & 15628 & 4795 & 20423 & 0.765 & 0.552 \\
\hline & 4 & 1 & $\mathrm{Y}$ & $\mathrm{N}$ & 28.55 & 31.85 & 290 & 37 & 326 & 0.888 & 0.264 \\
\hline & & 2 & $\mathrm{Y}$ & $\mathrm{N}$ & 29.61 & 33.31 & 141 & 13 & 155 & 0.913 & 0.194 \\
\hline & & 3 & $\mathrm{Y}$ & $\mathrm{N}$ & 19.33 & 22.88 & 155268 & 16667 & 171936 & 0.903 & 0.222 \\
\hline & 5 & 1 & $\mathrm{Y}$ & $\mathrm{N}$ & 24.02 & 27.7 & 6379 & 620 & 7000 & 0.911 & 0.198 \\
\hline & & 2 & $\mathrm{Y}$ & $\mathrm{N}$ & 25.83 & 29.73 & 1856 & 156 & 2012 & 0.923 & 0.165 \\
\hline & & 3 & $\mathrm{Y}$ & $\mathrm{N}$ & 24.96 & 28.15 & 3348 & 455 & 3804 & 0.88 & 0.286 \\
\hline North & 6 & 1 & $\mathrm{Y}$ & Y & 24.97 & 26.87 & 3329 & 1096 & 4426 & 0.752 & 0.576 \\
\hline \multirow[t]{14}{*}{ Bay } & & 2 & $\mathrm{Y}$ & $\mathrm{Y}$ & 24.02 & 25.85 & 6347 & 2188 & 8535 & 0.744 & 0.592 \\
\hline & & 3 & $\mathrm{Y}$ & $\mathrm{Y}$ & 23.42 & 25.25 & 9584 & 3295 & 12878 & 0.744 & 0.591 \\
\hline & 7 & 1 & $\mathrm{Y}$ & $\mathrm{N}$ & 26.8 & 30.26 & 958 & 108 & 1066 & 0.899 & 0.235 \\
\hline & & 2 & $\mathrm{Y}$ & $\mathrm{N}$ & 24.22 & 27.56 & 5540 & 683 & 6223 & 0.89 & 0.259 \\
\hline & & 3 & $\mathrm{Y}$ & $\mathrm{N}$ & 23.58 & 27.08 & 8588 & 947 & 9535 & 0.901 & 0.229 \\
\hline & 8 & 1 & $\mathrm{Y}$ & $\mathrm{N}$ & 20.95 & 24.51 & 51669 & 5464 & 57133 & 0.904 & 0.219 \\
\hline & & 2 & $\mathrm{Y}$ & $\mathrm{N}$ & 22.43 & 26.03 & 18771 & 1942 & 20714 & 0.906 & 0.213 \\
\hline & & 3 & $\mathrm{Y}$ & $\mathrm{N}$ & 19.97 & 23.23 & 100557 & 13159 & 113717 & 0.884 & 0.275 \\
\hline & 9 & 1 & $\mathrm{Y}$ & $\mathrm{Y}$ & 23.87 & 26.07 & 7059 & 1889 & 8948 & 0.789 & 0.505 \\
\hline & & 2 & $\mathrm{Y}$ & $\mathrm{N}$ & 23.94 & 26.44 & 6725 & 1468 & 8194 & 0.821 & 0.435 \\
\hline & & 3 & $\mathrm{Y}$ & $\mathrm{N}$ & 23.38 & 25.35 & 9822 & 3091 & 12913 & 0.761 & 0.561 \\
\hline & 10 & 1 & $\mathrm{Y}$ & $\mathrm{Y}$ & 20.98 & 22.99 & 50532 & 15412 & 65944 & 0.766 & 0.55 \\
\hline & & 2 & $\mathrm{Y}$ & $\mathrm{Y}$ & 24.43 & 26.25 & 4804 & 1672 & 6476 & 0.742 & 0.595 \\
\hline & & 3 & $\mathrm{Y}$ & $\mathrm{Y}$ & 20.18 & 22.12 & 86928 & 28009 & 114936 & 0.756 & 0.569 \\
\hline Comet's & 11 & 1 & $\mathrm{Y}$ & $\mathrm{N}$ & 24.86 & 27.08 & 3592 & 947 & 4539 & 0.791 & 0.5 \\
\hline \multirow[t]{2}{*}{ Hole } & & 2 & $\mathrm{Y}$ & $\mathrm{Y}$ & 22.33 & 24.58 & 20130 & 5223 & 25353 & 0.794 & 0.494 \\
\hline & & 3 & $\mathrm{Y}$ & $\mathrm{Y}$ & 22.1 & 24.26 & 23474 & 6483 & 29957 & 0.784 & 0.516 \\
\hline
\end{tabular}




\begin{tabular}{|c|c|c|c|c|c|c|c|c|c|c|c|}
\hline & 12 & 1 & $\mathrm{Y}$ & $\mathrm{N}$ & 25.35 & 28.56 & 2579 & 346 & 2925 & 0.882 & 0.282 \\
\hline & & 2 & $\mathrm{Y}$ & $\mathrm{N}$ & 20.42 & 24.04 & 73843 & 7566 & 81409 & 0.907 & 0.211 \\
\hline & & 3 & $\mathrm{Y}$ & $\mathrm{N}$ & 20.68 & 24.29 & 62054 & 6376 & 68429 & 0.907 & 0.212 \\
\hline & 13 & 1 & $\mathrm{Y}$ & $\mathrm{Y}$ & 23.83 & 26.01 & 7245 & 1961 & 9206 & 0.787 & 0.509 \\
\hline & & 2 & $\mathrm{Y}$ & $\mathrm{N}$ & 24.67 & 26.8 & 4099 & 1149 & 5248 & 0.781 & 0.521 \\
\hline & & 3 & $\mathrm{Y}$ & $\mathrm{Y}$ & 26.12 & 28.19 & 1525 & 444 & 1968 & 0.774 & 0.534 \\
\hline & 14 & 1 & $\mathrm{Y}$ & $\mathrm{N}$ & 23.46 & 26.48 & 9335 & 1426 & 10761 & 0.867 & 0.32 \\
\hline & & 2 & $\mathrm{Y}$ & $\mathrm{N}$ & 19.81 & 23.04 & 112118 & 14917 & 127035 & 0.883 & 0.28 \\
\hline & & 3 & $\mathrm{Y}$ & $\mathrm{N}$ & 19.28 & 22.43 & 161302 & 22583 & 183885 & 0.877 & 0.294 \\
\hline & 15 & 1 & $\mathrm{Y}$ & $\mathrm{Y}$ & 27.65 & 29.84 & 536 & 144 & 680 & 0.789 & 0.506 \\
\hline & & 2 & $\mathrm{Y}$ & $\mathrm{N}$ & 21.8 & 23.93 & 28813 & 8141 & 36954 & 0.78 & 0.524 \\
\hline & & 3 & $\mathrm{Y}$ & $\mathrm{N}$ & 20.82 & 23.03 & 56351 & 15078 & 71430 & 0.789 & 0.505 \\
\hline \multirow[t]{15}{*}{ The Arch } & 16 & 1 & $\mathrm{Y}$ & $\mathrm{N}$ & 23.18 & 26.02 & 11273 & 1949 & 13222 & 0.853 & 0.358 \\
\hline & & 2 & $\mathrm{Y}$ & $\mathrm{N}$ & 22.68 & 25.84 & 15821 & 2213 & 18034 & 0.877 & 0.294 \\
\hline & & 3 & $\mathrm{Y}$ & $\mathrm{N}$ & 22.6 & 25.62 & 16790 & 2572 & 19362 & 0.867 & 0.321 \\
\hline & 17 & 1 & $\mathrm{Y}$ & $\mathrm{Y}$ & 26.89 & 28.82 & 902 & 289 & 1191 & 0.757 & 0.567 \\
\hline & & 2 & $\mathrm{Y}$ & $\mathrm{Y}$ & 23.93 & 25.86 & 6770 & 2171 & 8941 & 0.757 & 0.567 \\
\hline & & 3 & $\mathrm{Y}$ & $\mathrm{Y}$ & 23.82 & 25.7 & 7288 & 2423 & 9711 & 0.75 & 0.58 \\
\hline & 18 & 1 & $\mathrm{Y}$ & Y & 23.82 & 25.21 & 7303 & 3399 & 10702 & 0.682 & 0.687 \\
\hline & & 2 & $\mathrm{Y}$ & $\mathrm{Y}$ & 24.25 & 25.67 & 5443 & 2481 & 7924 & 0.687 & 0.681 \\
\hline & & 3 & $\mathrm{Y}$ & $\mathrm{Y}$ & 22.44 & 23.86 & 18674 & 8506 & 27180 & 0.687 & 0.681 \\
\hline & 19 & 1 & $\mathrm{Y}$ & $\mathrm{Y}$ & 23.7 & 25.56 & 7906 & 2671 & 10576 & 0.747 & 0.585 \\
\hline & & 2 & $\mathrm{Y}$ & $\mathrm{N}$ & 29.48 & 31.84 & 154 & 37 & 191 & 0.808 & 0.463 \\
\hline & & 3 & $\mathrm{Y}$ & $\mathrm{N}$ & 26.05 & 28.05 & 1597 & 487 & 2084 & 0.766 & 0.55 \\
\hline & 20 & 1 & $\mathrm{Y}$ & $\mathrm{Y}$ & 24.4 & 25.56 & 4917 & 2664 & 7582 & 0.649 & 0.729 \\
\hline & & 2 & $\mathrm{Y}$ & $\mathrm{Y}$ & 25.46 & 26.77 & 2387 & 1169 & 3556 & 0.671 & 0.702 \\
\hline & & 3 & Y & $\mathrm{Y}$ & 25.82 & 27.19 & 1873 & 880 & 2753 & 0.68 & 0.69 \\
\hline \multirow[t]{8}{*}{ Malabar } & 21 & 1 & Y & $\mathrm{Y}$ & 20.21 & 22.15 & 85302 & 27453 & 112755 & 0.757 & 0.569 \\
\hline & & 2 & Y & $\mathrm{N}$ & 22.41 & 24.43 & 19120 & 5779 & 24899 & 0.768 & 0.547 \\
\hline & & 3 & Y & $\mathrm{Y}$ & 20.22 & 22.02 & 84787 & 30006 & 114793 & 0.739 & 0.601 \\
\hline & 22 & 1 & $\mathrm{Y}$ & $\mathrm{Y}$ & 20.22 & 22.5 & 84570 & 21636 & 106206 & 0.796 & 0.489 \\
\hline & & 2 & Y & $\mathrm{N}$ & 24.41 & 26.97 & 4879 & 1020 & 5899 & 0.827 & 0.42 \\
\hline & & 3 & $\mathrm{Y}$ & $\mathrm{N}$ & 21.75 & 24.28 & 29954 & 6397 & 36351 & 0.824 & 0.428 \\
\hline & 23 & 1 & Y & $\mathrm{Y}$ & 21.88 & 23.82 & 27294 & 8748 & 36042 & 0.757 & 0.567 \\
\hline & & 2 & $\mathrm{Y}$ & $\mathrm{Y}$ & 19.61 & 21.76 & 128258 & 35751 & 164009 & 0.782 & 0.519 \\
\hline
\end{tabular}




\begin{tabular}{|c|c|c|c|c|c|c|c|c|c|c|c|}
\hline & & 3 & $\mathrm{Y}$ & $\mathrm{Y}$ & 20.04 & 22.09 & 95688 & 28596 & 124284 & 0.77 & 0.543 \\
\hline & 24 & 1 & $\mathrm{Y}$ & Y & 22.62 & 24.6 & 16492 & 5157 & 21648 & 0.762 & 0.559 \\
\hline & & 2 & $\mathrm{Y}$ & $\mathrm{Y}$ & 20.59 & 22.63 & 65731 & 19824 & 85555 & 0.768 & 0.546 \\
\hline & & 3 & $\mathrm{Y}$ & $\mathrm{N}$ & 19.9 & 22.25 & 105462 & 25629 & 131091 & 0.804 & 0.472 \\
\hline & 25 & 1 & $\mathrm{Y}$ & $\mathrm{Y}$ & 21.92 & 23.76 & 26660 & 9119 & 35779 & 0.745 & 0.589 \\
\hline & & 2 & $\mathrm{Y}$ & $\mathrm{N}$ & 20.55 & 22.53 & 67606 & 21174 & 88780 & 0.761 & 0.559 \\
\hline & & 3 & $\mathrm{Y}$ & $\mathrm{Y}$ & 23.33 & 25.12 & 10159 & 3618 & 13777 & 0.737 & 0.603 \\
\hline Sugarloaf & 26 & 1 & $\mathrm{Y}$ & $\mathrm{Y}$ & 24.12 & 26.01 & 5944 & 1963 & 7907 & 0.752 & 0.577 \\
\hline \multirow[t]{14}{*}{ West } & & 2 & $\mathrm{Y}$ & $\mathrm{Y}$ & 25.46 & 27.31 & 2386 & 811 & 3197 & 0.746 & 0.587 \\
\hline & & 3 & $\mathrm{Y}$ & $\mathrm{Y}$ & 23.19 & 25.21 & 11177 & 3394 & 14571 & 0.767 & 0.549 \\
\hline & 27 & 1 & $\mathrm{Y}$ & $\mathrm{Y}$ & 25.97 & 27.7 & 1690 & 622 & 2311 & 0.731 & 0.614 \\
\hline & & 2 & $\mathrm{Y}$ & $\mathrm{Y}$ & 24.19 & 25.66 & 5664 & 2499 & 8163 & 0.694 & 0.671 \\
\hline & & 3 & $\mathrm{Y}$ & $\mathrm{Y}$ & 23.61 & 25.09 & 8396 & 3682 & 12078 & 0.695 & 0.67 \\
\hline & 28 & 1 & $\mathrm{Y}$ & $\mathrm{Y}$ & 29.23 & 31.14 & 182 & 59 & 241 & 0.755 & 0.572 \\
\hline & & 2 & $\mathrm{Y}$ & $\mathrm{Y}$ & 26.25 & 27.96 & 1388 & 520 & 1908 & 0.728 & 0.619 \\
\hline & & 3 & $\mathrm{Y}$ & $\mathrm{Y}$ & 28.13 & 30.21 & 388 & 112 & 499 & 0.777 & 0.53 \\
\hline & 29 & 1 & $\mathrm{Y}$ & $\mathrm{N}$ & 23.61 & 25.89 & 8419 & 2130 & 10549 & 0.798 & 0.486 \\
\hline & & 2 & $\mathrm{Y}$ & $\mathrm{N}$ & 24.45 & 26.89 & 4749 & 1078 & 5827 & 0.815 & 0.448 \\
\hline & & 3 & $\mathrm{Y}$ & $\mathrm{N}$ & 25.57 & 28.06 & 2218 & 486 & 2705 & 0.82 & 0.436 \\
\hline & 30 & 1 & $\mathrm{Y}$ & $\mathrm{Y}$ & 23.62 & 25.06 & 8384 & 3757 & 12141 & 0.691 & 0.676 \\
\hline & & 2 & $\mathrm{Y}$ & $\mathrm{Y}$ & 20.98 & 22.48 & 50567 & 21956 & 72523 & 0.697 & 0.667 \\
\hline & & 3 & $\mathrm{Y}$ & $\mathrm{Y}$ & 23.51 & 25.24 & 9013 & 3334 & 12347 & 0.73 & 0.615 \\
\hline Ned's & 31 & 1 & $\mathrm{Y}$ & $\mathrm{Y}$ & 22.18 & 23.91 & 22299 & 8260 & 30559 & 0.73 & 0.616 \\
\hline \multirow[t]{12}{*}{ Beach } & & 2 & $\mathrm{Y}$ & $\mathrm{Y}$ & 22.51 & 23.94 & 17835 & 8098 & 25933 & 0.688 & 0.68 \\
\hline & & 3 & $\mathrm{Y}$ & $\mathrm{Y}$ & 21.8 & 23.38 & 28896 & 11878 & 40775 & 0.709 & 0.65 \\
\hline & 32 & 1 & $\mathrm{Y}$ & $\mathrm{Y}$ & 24.84 & 25.2 & 3651 & 3424 & 7075 & 0.516 & 0.816 \\
\hline & & 2 & $\mathrm{Y}$ & $\mathrm{Y}$ & 26.96 & 27.41 & 861 & 759 & 1620 & 0.532 & 0.812 \\
\hline & & 3 & Y & $\mathrm{Y}$ & 23.4 & 23.86 & 9744 & 8561 & 18305 & 0.532 & 0.812 \\
\hline & 33 & 1 & $\mathrm{Y}$ & $\mathrm{N}$ & 21.65 & 24.39 & 31960 & 5936 & 37897 & 0.843 & 0.381 \\
\hline & & 2 & $\mathrm{Y}$ & $\mathrm{N}$ & 25.06 & 27.64 & 3141 & 645 & 3786 & 0.83 & 0.414 \\
\hline & & 3 & $\mathrm{Y}$ & $\mathrm{N}$ & 24.06 & 26.8 & 6204 & 1144 & 7349 & 0.844 & 0.379 \\
\hline & 34 & 1 & $\mathrm{Y}$ & $\mathrm{Y}$ & 22.34 & 23.89 & 19933 & 8366 & 28299 & 0.704 & 0.656 \\
\hline & & 2 & Y & $\mathrm{Y}$ & 26.6 & 27.9 & 1098 & 542 & 1640 & 0.669 & 0.704 \\
\hline & & 3 & Y & $\mathrm{Y}$ & 25.15 & 26.34 & 2946 & 1574 & 4520 & 0.652 & 0.725 \\
\hline & 35 & 1 & Y & $\mathrm{Y}$ & 21.72 & 23.97 & 30438 & 7936 & 38374 & 0.793 & 0.496 \\
\hline
\end{tabular}




\begin{tabular}{|c|c|c|c|c|c|c|c|c|c|c|c|}
\hline & & 2 & $\mathrm{Y}$ & $\mathrm{N}$ & 24.03 & 26.23 & 6313 & 1694 & 8007 & 0.788 & 0.506 \\
\hline & & 3 & $\mathrm{Y}$ & $\mathrm{Y}$ & 23.29 & 25.28 & 10464 & 3231 & 13695 & 0.764 & 0.554 \\
\hline Algal & 36 & 1 & $\mathrm{Y}$ & $\mathrm{Y}$ & 22.31 & 24.12 & 20411 & 7165 & 27576 & 0.74 & 0.598 \\
\hline Hole & & 2 & $\mathrm{Y}$ & $\mathrm{Y}$ & 22.48 & 24.38 & 18165 & 5986 & 24150 & 0.752 & 0.577 \\
\hline \multirow[t]{13}{*}{ North } & & 3 & $\mathrm{Y}$ & $\mathrm{Y}$ & 25.4 & 27.36 & 2480 & 780 & 3260 & 0.761 & 0.561 \\
\hline & 37 & 1 & $\mathrm{Y}$ & $\mathrm{Y}$ & 24.48 & 26.14 & 4661 & 1797 & 6458 & 0.722 & 0.629 \\
\hline & & 2 & $\mathrm{Y}$ & $\mathrm{Y}$ & 23.09 & 24.76 & 11959 & 4614 & 16573 & 0.722 & 0.629 \\
\hline & & 3 & $\mathrm{Y}$ & $\mathrm{Y}$ & 22.99 & 24.52 & 12878 & 5436 & 18314 & 0.703 & 0.658 \\
\hline & 38 & 1 & $\mathrm{Y}$ & $\mathrm{Y}$ & 21.16 & 23.3 & 44704 & 12503 & 57207 & 0.781 & 0.52 \\
\hline & & 2 & $\mathrm{Y}$ & $\mathrm{Y}$ & 22.53 & 24.65 & 17613 & 4983 & 22596 & 0.779 & 0.524 \\
\hline & & 3 & Y & Y & 19.95 & 21.99 & 101930 & 30586 & 132516 & 0.769 & 0.545 \\
\hline & 39 & 1 & $\mathrm{Y}$ & $\mathrm{N}$ & 20.62 & 22.96 & 64765 & 15802 & 80568 & 0.804 & 0.473 \\
\hline & & 2 & $\mathrm{Y}$ & $\mathrm{N}$ & 21.47 & 23.79 & 36084 & 8931 & 45015 & 0.802 & 0.478 \\
\hline & & 3 & $\mathrm{Y}$ & $\mathrm{N}$ & 21.93 & 24.45 & 26359 & 5692 & 32051 & 0.822 & 0.431 \\
\hline & 40 & 1 & $\mathrm{Y}$ & $\mathrm{N}$ & 21.38 & 23.72 & 38501 & 9381 & 47881 & 0.804 & 0.472 \\
\hline & & 2 & $\mathrm{Y}$ & $\mathrm{N}$ & 22.39 & 24.92 & 19308 & 4138 & 23447 & 0.824 & 0.429 \\
\hline & & 3 & $\mathrm{Y}$ & $\mathrm{N}$ & 18.3 & 20.74 & 313079 & 71992 & 385071 & 0.813 & 0.453 \\
\hline
\end{tabular}


Table B.12 Variation in $S_{\mathrm{H}}: S_{\mathrm{T}}$ along a depth gradient at The Arch (chapter 3)

\begin{tabular}{|c|c|c|c|c|c|c|c|c|c|c|}
\hline ID & Depth (m) & C100 band & C109 band & Mean $C_{t}\left(\mathrm{C100}^{+}\right)$ & Mean $C_{t}\left(\mathrm{C} 100^{-}\right)$ & $\mathrm{C}_{\mathrm{C} 100}$ & $\mathrm{C}_{\mathrm{C} 100}{ }^{-}$ & $\mathbf{C}_{\text {TOTAL }}$ & $\mathrm{C}_{\mathrm{C} 100}: \mathrm{C}_{\mathrm{TOTAL}}$ & $S_{\mathrm{H}}: S_{\mathrm{T}}$ \\
\hline 1 & 9.5 & $\mathrm{Y}$ & $\mathrm{Y}$ & 21.24 & 23.18 & 42460 & 13556 & 56016 & 0.758 & 0.566 \\
\hline 2 & 7.5 & $\mathrm{Y}$ & $\mathrm{N}$ & 22.16 & 25.32 & 22613 & 3152 & 25765 & 0.878 & 0.293 \\
\hline 3 & 5.1 & $\mathrm{Y}$ & $\mathrm{Y}$ & 20.6 & 22.47 & 65548 & 21976 & 87524 & 0.749 & 0.583 \\
\hline 4 & 4.4 & $\mathrm{Y}$ & $\mathrm{N}$ & 26.12 & 28.44 & 1521 & 375 & 1896 & 0.802 & 0.477 \\
\hline 5 & 4.4 & $\mathrm{Y}$ & $\mathrm{Y}$ & 22.34 & 24.72 & 20025 & 4753 & 24778 & 0.808 & 0.463 \\
\hline 6 & 9.7 & $\mathrm{Y}$ & $\mathrm{N}$ & 21.04 & 23.64 & 48649 & 9939 & 58588 & 0.83 & 0.413 \\
\hline 7 & 7.5 & $\mathrm{Y}$ & $\mathrm{Y}$ & 23.16 & 25.3 & 11401 & 3190 & 14591 & 0.781 & 0.52 \\
\hline 8 & 5.4 & $\mathrm{Y}$ & $\mathrm{N}$ & 19.34 & 22.53 & 154749 & 21089 & 175838 & 0.88 & 0.287 \\
\hline 9 & 8.8 & $\mathrm{Y}$ & $\mathrm{Y}$ & 20.3 & 22.23 & 80366 & 25883 & 106249 & 0.756 & 0.569 \\
\hline 10 & 11 & $\mathrm{Y}$ & $\mathrm{N}$ & 23.77 & 26.49 & 7542 & 1421 & 8963 & 0.841 & 0.386 \\
\hline 11 & 11.6 & Y & $\mathrm{Y}$ & 25.2 & 27.05 & 2854 & 966 & 3820 & 0.747 & 0.586 \\
\hline 12 & 4.4 & $\mathrm{Y}$ & $\mathrm{N}$ & 27.34 & 29.49 & 662 & 183 & 844 & 0.784 & 0.516 \\
\hline 13 & 4.7 & $\mathrm{Y}$ & $\mathrm{Y}$ & 20.12 & 22.16 & 90742 & 27213 & 117955 & 0.769 & 0.544 \\
\hline 14 & 4.8 & $\mathrm{Y}$ & $\mathrm{N}$ & 20.96 & 23.65 & 51184 & 9847 & 61030 & 0.839 & 0.393 \\
\hline 15 & 10.8 & $\mathrm{Y}$ & $\mathrm{Y}$ & 22.26 & 24.69 & 21140 & 4856 & 25997 & 0.813 & 0.452 \\
\hline 16 & 6.8 & $\mathrm{Y}$ & $\mathrm{Y}$ & 21.82 & 23.05 & 28543 & 14876 & 43419 & 0.657 & 0.719 \\
\hline $17 *$ & 9.9 & Y & $\mathrm{N}$ & 23.18 & 26.02 & 11273 & 1949 & 13222 & 0.853 & 0.358 \\
\hline $18^{*}$ & 11.1 & $\mathrm{Y}$ & $\mathrm{Y}$ & 26.89 & 28.82 & 902 & 289 & 1191 & 0.757 & 0.567 \\
\hline $19 *$ & 10.2 & $\mathrm{Y}$ & $\mathrm{Y}$ & 23.82 & 25.21 & 7303 & 3399 & 10702 & 0.682 & 0.687 \\
\hline $20 *$ & 10.6 & $\mathrm{Y}$ & $\mathrm{Y}$ & 23.7 & 25.56 & 7906 & 2671 & 10576 & 0.747 & 0.585 \\
\hline $21 *$ & 11.6 & $\mathrm{Y}$ & $\mathrm{Y}$ & 24.4 & 25.56 & 4917 & 2664 & 7582 & 0.649 & 0.729 \\
\hline 22 & 9.9 & $\mathrm{Y}$ & $\mathrm{Y}$ & 21.86 & 23.76 & 27675 & 9134 & 36809 & 0.752 & 0.577 \\
\hline 23 & 11.2 & $\mathrm{Y}$ & $\mathrm{Y}$ & 23.44 & 25.07 & 9442 & 3724 & 13167 & 0.717 & 0.636 \\
\hline 24 & 10.5 & $\mathrm{Y}$ & $\mathrm{Y}$ & 22.36 & 23.7 & 19756 & 9532 & 29288 & 0.675 & 0.698 \\
\hline 25 & 11 & $\mathrm{Y}$ & $\mathrm{Y}$ & 22.65 & 23.99 & 16239 & 7797 & 24036 & 0.676 & 0.696 \\
\hline 26 & 10.4 & $\mathrm{Y}$ & $\mathrm{N}$ & 23.06 & 26.18 & 12250 & 1756 & 14006 & 0.875 & 0.301 \\
\hline
\end{tabular}


Table B.13 Longitudinal analysis of temporal variation in $S_{\mathrm{H}}: S_{\mathrm{T}}$ (chapter 3)

\begin{tabular}{|c|c|c|c|c|c|c|c|c|c|}
\hline Site & ID & Time & $\mathrm{C}_{\mathrm{t}}\left(\mathrm{C100}^{+}\right)$ & $\mathrm{C}_{\mathrm{t}}\left(\mathrm{C100}^{-}\right)$ & $\mathrm{C}_{\mathrm{C} 100}$ & $\mathrm{C}_{\mathrm{C} 100}{ }^{-}$ & $\mathbf{C}_{\text {TOTAL }}$ & $\mathrm{C}_{100}: \mathrm{C}_{\text {TOTAL }}$ & $S_{\mathrm{H}}: S_{\mathrm{T}}$ \\
\hline Comet's & 1 & Mar 2011 & 22.41 & 24.63 & 19090 & 5030 & 24119 & 0.791 & 0.5 \\
\hline \multirow[t]{19}{*}{ Hole } & & Sep 2011 & 24.86 & 27.08 & 3592 & 947 & 4539 & 0.791 & 0.5 \\
\hline & & Mar 2012 & 19.74 & 21.97 & 117800 & 31077 & 148877 & 0.791 & 0.5 \\
\hline & & Sep 2012 & 18.68 & 20.94 & 242570 & 62740 & 305310 & 0.795 & 0.493 \\
\hline & 2 & Mar 2011 & 20.88 & 24.06 & 54042 & 7424 & 61466 & 0.879 & 0.289 \\
\hline & & Sep 2011 & 25.35 & 28.56 & 2579 & 346 & 2925 & 0.882 & 0.282 \\
\hline & & Mar 2012 & 20.93 & 24.41 & 52327 & 5880 & 58207 & 0.899 & 0.234 \\
\hline & & Sep 2012 & 19.38 & 22.89 & 150383 & 16518 & 166902 & 0.901 & 0.228 \\
\hline & 3 & Mar 2011 & 27.8 & 30.07 & 485 & 123 & 608 & 0.797 & 0.487 \\
\hline & & Sep 2011 & 23.83 & 26.01 & 7245 & 1961 & 9206 & 0.787 & 0.509 \\
\hline & & Mar 2012 & 23.57 & 25.96 & 8675 & 2040 & 10715 & 0.81 & 0.46 \\
\hline & & Sep 2012 & 20.83 & 23.16 & 55972 & 13777 & 69749 & 0.802 & 0.476 \\
\hline & 4 & Mar 2011 & 19.14 & 22.42 & 177333 & 22833 & 200166 & 0.886 & 0.271 \\
\hline & & Sep 2011 & 23.46 & 26.48 & 9335 & 1426 & 10761 & 0.867 & 0.32 \\
\hline & & Mar 2012 & 21.26 & 24.24 & 41778 & 6560 & 48338 & 0.864 & 0.328 \\
\hline & & Sep 2012 & 20.56 & 23.78 & 67451 & 8993 & 76444 & 0.882 & 0.28 \\
\hline & 5 & Mar 2011 & 21.44 & 23.79 & 36954 & 8920 & 45875 & 0.806 & 0.469 \\
\hline & & Sep 2011 & 27.65 & 29.84 & 536 & 144 & 680 & 0.789 & 0.506 \\
\hline & & Mar 2012 & 21.82 & 24.3 & 28414 & 6301 & 34715 & 0.818 & 0.44 \\
\hline & & Sep 2012 & 20.59 & 22.65 & 65985 & 19505 & 85491 & 0.772 & 0.539 \\
\hline North & 6 & Mar 2011 & 22.37 & 24.26 & 19579 & 6514 & 26093 & 0.75 & 0.58 \\
\hline \multirow[t]{12}{*}{ Bay } & & Sep 2011 & 23.42 & 25.25 & 9584 & 3295 & 12878 & 0.744 & 0.591 \\
\hline & & Mar 2012 & 24.9 & 26.77 & 3500 & 1174 & 4673 & 0.749 & 0.583 \\
\hline & & Sep 2012 & 21.82 & 23.53 & 28446 & 10662 & 39109 & 0.727 & 0.62 \\
\hline & 7 & Mar 2011 & 23.5 & 27.17 & 9047 & 889 & 9935 & 0.911 & 0.201 \\
\hline & & Sep 2011 & 26.8 & 30.26 & 958 & 108 & 1066 & 0.899 & 0.235 \\
\hline & & Mar 2012 & 24.29 & 27.51 & 5288 & 706 & 5995 & 0.882 & 0.281 \\
\hline & & Sep 2012 & 24.36 & 27.59 & 5057 & 668 & 5725 & 0.883 & 0.278 \\
\hline & 8 & Mar 2011 & 19.95 & 23.65 & 101785 & 9855 & 111640 & 0.912 & 0.197 \\
\hline & & Sep 2011 & 20.95 & 24.51 & 51669 & 5464 & 57133 & 0.904 & 0.219 \\
\hline & & Mar 2012 & 21.09 & 24.34 & 46818 & 6158 & 52976 & 0.884 & 0.276 \\
\hline & & Sep 2012 & 22.51 & 26 & 17804 & 1980 & 19784 & 0.9 & 0.231 \\
\hline & 9 & Mar 2011 & 24.86 & 27.07 & 3582 & 951 & 4533 & 0.79 & 0.502 \\
\hline
\end{tabular}




\begin{tabular}{|c|c|c|c|c|c|c|c|c|c|}
\hline & & Sep 2011 & 23.87 & 26.07 & 7059 & 1889 & 8948 & 0.789 & 0.505 \\
\hline & & Mar 2012 & 21.31 & 23.65 & 40406 & 9819 & 50225 & 0.804 & 0.472 \\
\hline & & Sep 2012 & 21.07 & 23.49 & 47457 & 10949 & 58406 & 0.813 & 0.454 \\
\hline & 10 & Mar 2011 & 23.06 & 25.26 & 12258 & 3272 & 15531 & 0.789 & 0.504 \\
\hline & & Sep 2011 & 20.98 & 22.99 & 50532 & 15412 & 65944 & 0.766 & 0.55 \\
\hline & & Mar 2012 & 24.37 & 26.36 & 5019 & 1551 & 6569 & 0.764 & 0.555 \\
\hline & & Sep 2012 & 23.75 & 25.87 & 7654 & 2164 & 9818 & 0.78 & 0.524 \\
\hline Sylph's & 11 & Mar 2011 & 22.3 & 26.37 & 20525 & 1539 & 22064 & 0.93 & 0.142 \\
\hline \multirow[t]{18}{*}{ Hole } & & Sep 2011 & 21.62 & 25.6 & 32698 & 2609 & 35306 & 0.926 & 0.154 \\
\hline & & Mar 2012 & 20.56 & 24.49 & 67320 & 5550 & 72870 & 0.924 & 0.161 \\
\hline & & Sep 2012 & 18.14 & 21.94 & 350788 & 31739 & 382527 & 0.917 & 0.182 \\
\hline & 12 & Mar 2011 & 25.97 & 28.12 & 1689 & 467 & 2156 & 0.784 & 0.516 \\
\hline & & Sep 2011 & 25.63 & 27.89 & 2128 & 545 & 2673 & 0.796 & 0.49 \\
\hline & & Mar 2012 & 19.35 & 21.56 & 153834 & 41070 & 194903 & 0.789 & 0.504 \\
\hline & & Sep 2012 & 20.98 & 23.19 & 50579 & 13482 & 64061 & 0.79 & 0.504 \\
\hline & 13 & Mar 2011 & 27.15 & 29.3 & 754 & 208 & 961 & 0.784 & 0.515 \\
\hline & & Sep 2011 & 27.33 & 29.28 & 666 & 210 & 876 & 0.76 & 0.562 \\
\hline & & Mar 2012 & 24.27 & 26.47 & 5358 & 1437 & 6795 & 0.788 & 0.506 \\
\hline & & Sep 2012 & 19.57 & 21.66 & 132194 & 38247 & 170441 & 0.776 & 0.532 \\
\hline & 14 & Mar 2011 & 25.08 & 28.4 & 3093 & 385 & 3477 & 0.889 & 0.261 \\
\hline & & Sep 2011 & 19.33 & 22.88 & 155268 & 16667 & 171936 & 0.903 & 0.222 \\
\hline & & Mar 2012 & 23.22 & 26.83 & 10997 & 1127 & 12124 & 0.907 & 0.211 \\
\hline & & Sep 2012 & 19.49 & 23.03 & 139659 & 15004 & 154663 & 0.903 & 0.223 \\
\hline & 15 & Mar 2011 & 21.17 & 25.08 & 44297 & 3716 & 48013 & 0.923 & 0.165 \\
\hline & & Sep 2011 & 24.02 & 27.7 & 6379 & 620 & 7000 & 0.911 & 0.198 \\
\hline & & Mar 2012 & 22.54 & 26.43 & 17497 & 1478 & 18975 & 0.922 & 0.166 \\
\hline
\end{tabular}




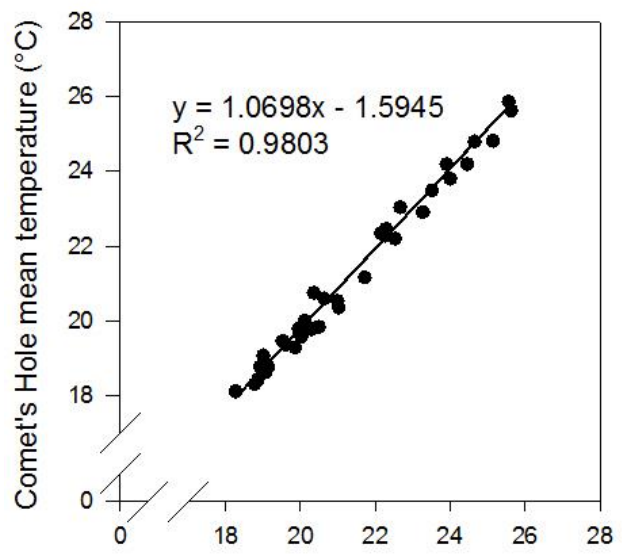

Algal Holes mean temperature $\left({ }^{\circ} \mathrm{C}\right)$

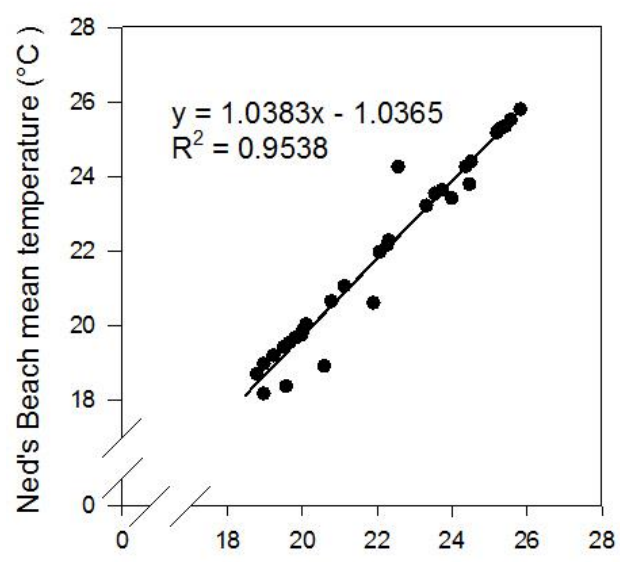

Sugarloaf West mean temperature $\left({ }^{\circ} \mathrm{C}\right)$

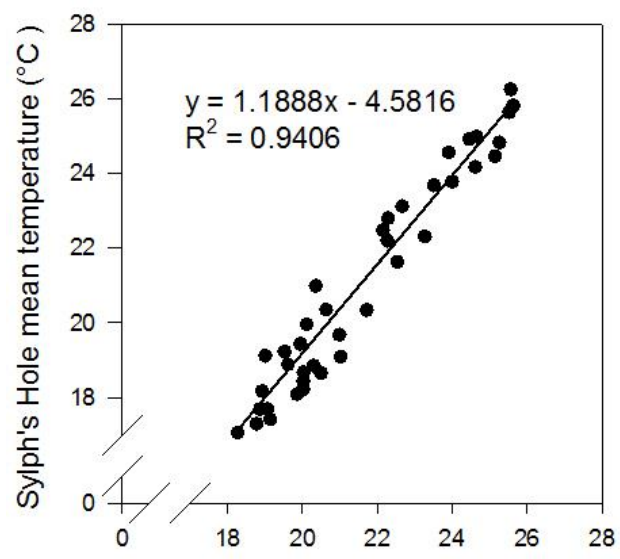

Algal Holes mean temperature $\left({ }^{\circ} \mathrm{C}\right)$

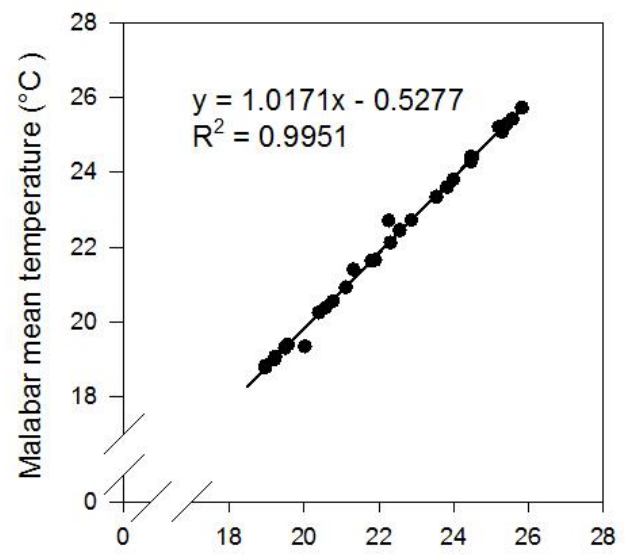

Sugarloaf West mean temperature $\left({ }^{\circ} \mathrm{C}\right)$

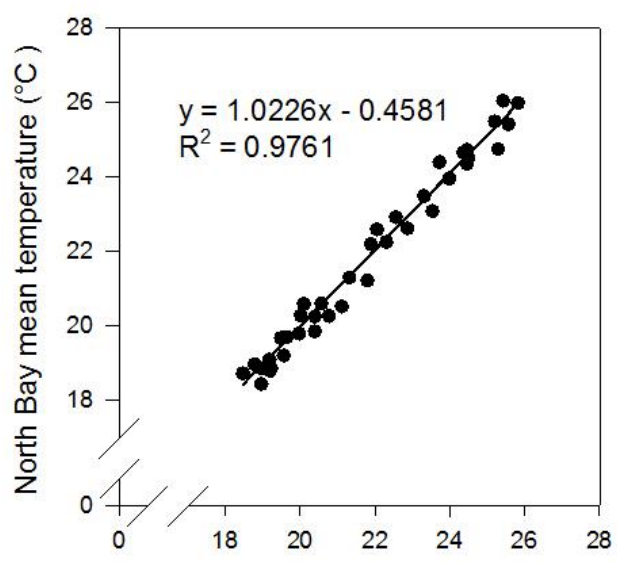

Sugarloaf West mean temperature $\left({ }^{\circ} \mathrm{C}\right)$

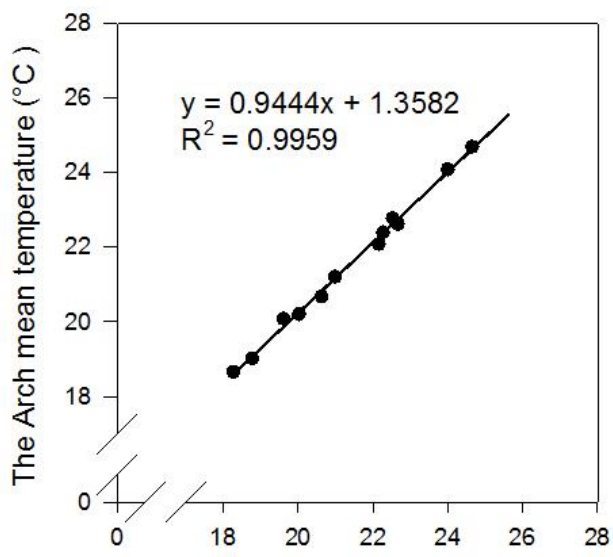

Algal Holes mean temperature $\left({ }^{\circ} \mathrm{C}\right)$

Figure B.2 Site-correlation to estimate missing temperature data (chapter 3)

Donor sites were either Algal Holes or Sugarloaf West, both of which had continuous temperature measurements available over the entire period. Circles represent monthly average temperatures during periods of simultaneous logging. Following correlation analysis, the parameters of the fitted line were used to predict missing monthly-temperature values. 
Table B.14 Climate model parameters for eight LHI study sites (chapter 3)

\begin{tabular}{lllllllll}
\hline Site & $\mathbf{a}$ & $\mathbf{b}$ & $\mathbf{c}$ & $\mathbf{d}$ & $\mathbf{d}+\mathbf{a}$ & $\mathbf{d}-\mathbf{a}$ & $\mathbf{2 a}$ & $\mathbf{R}^{\mathbf{2}}$ \\
\hline Algal Holes & 3.052 & 11.977 & 1.537 & 21.841 & 24.893 & 18.789 & 6.104 & 0.91 \\
Comet's Hole & 3.271 & 11.985 & -4.659 & 21.72 & 24.991 & 18.45 & 6.541 & 0.912 \\
Malabar & 3.079 & 11.997 & 1.472 & 21.683 & 24.762 & 18.604 & 6.158 & 0.909 \\
Ned's Beach & 3.217 & 12.007 & 1.48 & 21.637 & 24.854 & 18.42 & 6.434 & 0.919 \\
North Bay & 3.159 & 12.07 & -4.627 & 21.844 & 25.003 & 18.686 & 6.317 & 0.909 \\
Sugarloaf West & 3.024 & 11.996 & 1.489 & 21.842 & 24.866 & 18.817 & 6.048 & 0.91 \\
Sylph's Hole & 3.811 & 12.021 & 1.788 & 21.366 & 25.177 & 17.555 & 7.621 & 0.918 \\
The Arch & 2.883 & 11.992 & 1.544 & 21.975 & 24.857 & 19.092 & 5.765 & 0.909 \\
\hline
\end{tabular}

The four sine-function parameters $\mathrm{a}, \mathrm{b}, \mathrm{c}$ and $\mathrm{d}$ represent the amplitude, period, phase shift and vertical shift, respectively. Parameter information can be interpreted as: $d$, mean temperature; $d+a$, temperature maximum; $\mathrm{d}-\mathrm{a}$, temperature minimum; $2 \mathrm{a}$, seasonal temperature variability. 
Table B.15 Calculation of $S_{\mathrm{H}}: S_{\mathrm{T}}$ ratios for physiology analysis (chapter 4)

\begin{tabular}{|c|c|c|c|c|c|c|c|c|c|c|}
\hline Fragment no. & Colony no. & Temperature & C100 band & C109 band & $\mathrm{C}_{\mathrm{t}}\left(\mathrm{C100}^{+}\right)$ & $\mathrm{C}_{\mathrm{t}}\left(\mathrm{C100}^{-}\right)$ & $\mathrm{C}_{\mathrm{C} 100}$ & $\mathbf{C}_{\text {TOTAL }}$ & $\mathrm{C}_{\mathrm{C} 100}: \mathrm{C}_{\mathrm{TOTAL}}$ & $S_{\mathrm{H}}: S_{\mathrm{T}}$ \\
\hline 1 & 1 & $25^{\circ} \mathrm{C}$ & $\mathrm{Y}$ & $\mathrm{Y}$ & 22.55 & 24 & 17284 & 25024 & 0.691 & 0.676 \\
\hline 2 & 2 & $25^{\circ} \mathrm{C}$ & Y & Y & 22.43 & 23.73 & 18871 & 28172 & 0.67 & 0.704 \\
\hline 3 & 3 & $25^{\circ} \mathrm{C}$ & $\mathrm{Y}$ & $\mathrm{N}$ & 22.16 & 24.52 & 22567 & 28021 & 0.805 & 0.47 \\
\hline 4 & 4 & $25^{\circ} \mathrm{C}$ & $\mathrm{Y}$ & $\mathrm{N}$ & 22.93 & 24.88 & 13362 & 17614 & 0.759 & 0.565 \\
\hline 5 & 5 & $25^{\circ} \mathrm{C}$ & $\mathrm{Y}$ & $\mathrm{Y}$ & 24.49 & 26.52 & 4622 & 6011 & 0.769 & 0.545 \\
\hline 6 & 6 & $25^{\circ} \mathrm{C}$ & $\mathrm{Y}$ & Y & 23.62 & 25.25 & 8337 & 11632 & 0.717 & 0.637 \\
\hline 7 & 7 & $25^{\circ} \mathrm{C}$ & Y & $\mathrm{N}$ & 21.94 & 25.05 & 26269 & 30049 & 0.874 & 0.302 \\
\hline 8 & 8 & $25^{\circ} \mathrm{C}$ & Y & Y & 22.45 & 24.11 & 18562 & 25734 & 0.721 & 0.63 \\
\hline 9 & 9 & $25^{\circ} \mathrm{C}$ & Y & Y & 24.52 & 26.75 & 4526 & 5709 & 0.793 & 0.497 \\
\hline 10 & 10 & $25^{\circ} \mathrm{C}$ & $\mathrm{Y}$ & $\mathrm{N}$ & 22.82 & 25.18 & 14422 & 17892 & 0.806 & 0.468 \\
\hline 11 & 11 & $25^{\circ} \mathrm{C}$ & Y & $\mathrm{N}$ & 23.89 & 25.96 & 6942 & 8979 & 0.773 & 0.537 \\
\hline 12 & 12 & $25^{\circ} \mathrm{C}$ & $\mathrm{Y}$ & $\mathrm{N}$ & 21.99 & 25.39 & 25410 & 28411 & 0.894 & 0.247 \\
\hline 13 & 13 & $25^{\circ} \mathrm{C}$ & Y & $\mathrm{N}$ & 22.52 & 26.23 & 17687 & 19376 & 0.913 & 0.194 \\
\hline 14 & 14 & $25^{\circ} \mathrm{C}$ & Y & $\mathrm{N}$ & 21.52 & 24.76 & 34956 & 39569 & 0.883 & 0.277 \\
\hline 15 & 15 & $25^{\circ} \mathrm{C}$ & Y & $\mathrm{N}$ & 20.94 & 24.61 & 51927 & 57050 & 0.91 & 0.202 \\
\hline 16 & 1 & $29^{\circ} \mathrm{C}$ & Y & Y & 24.1 & 25.45 & 6018 & 8908 & 0.676 & 0.696 \\
\hline 17 & 2 & $29^{\circ} \mathrm{C}$ & Y & Y & 23.69 & 24.93 & 7992 & 12106 & 0.66 & 0.716 \\
\hline 18 & 3 & $29^{\circ} \mathrm{C}$ & Y & $\mathrm{N}$ & 22.58 & 25.02 & 17004 & 20863 & 0.815 & 0.448 \\
\hline 19 & 4 & $29^{\circ} \mathrm{C}$ & $Y$ & $\mathrm{~N}$ & 23.36 & 25.42 & 9987 & 12930 & 0.772 & 0.538 \\
\hline 20 & 5 & $29^{\circ} \mathrm{C}$ & Y & Y & 24.63 & 26.65 & 4196 & 5464 & 0.768 & 0.547 \\
\hline 21 & 6 & $29^{\circ} \mathrm{C}$ & Y & Y & 23.39 & 25.12 & 9784 & 13403 & 0.73 & 0.616 \\
\hline 22 & 7 & $29^{\circ} \mathrm{C}$ & Y & $\mathrm{N}$ & 21.02 & 25.03 & 49136 & 52979 & 0.927 & 0.15 \\
\hline 23 & 8 & $29^{\circ} \mathrm{C}$ & $Y$ & $Y$ & 22.21 & 23.97 & 21872 & 29775 & 0.735 & 0.608 \\
\hline 24 & 9 & $29^{\circ} \mathrm{C}$ & $\mathrm{Y}$ & $\mathrm{N}$ & 24 & 25.95 & 6454 & 8498 & 0.759 & 0.563 \\
\hline 25 & 10 & $29^{\circ} \mathrm{C}$ & Y & $\mathrm{N}$ & 23.95 & 26.3 & 6688 & 8303 & 0.805 & 0.47 \\
\hline 26 & 11 & $29^{\circ} \mathrm{C}$ & Y & $\mathrm{N}$ & 24.22 & 26.18 & 5552 & 7306 & 0.76 & 0.562 \\
\hline 27 & 12 & $29^{\circ} \mathrm{C}$ & $\mathrm{Y}$ & $\mathrm{N}$ & 24.2 & 27.66 & 5617 & 6255 & 0.898 & 0.237 \\
\hline 28 & 13 & $29^{\circ} \mathrm{C}$ & $\mathrm{Y}$ & $\mathrm{N}$ & 23.48 & 26.83 & 9200 & 10320 & 0.891 & 0.255 \\
\hline 29 & 14 & $29^{\circ} \mathrm{C}$ & $\mathrm{Y}$ & $\mathrm{N}$ & 22.2 & 25.53 & 22071 & 24803 & 0.89 & 0.26 \\
\hline 30 & 15 & $29^{\circ} \mathrm{C}$ & Y & $\mathrm{N}$ & 21.84 & 25.38 & 28141 & 31168 & 0.903 & 0.223 \\
\hline
\end{tabular}


Table B.16 Physiological diagnostics (chapter 4)

\begin{tabular}{|c|c|c|c|c|c|c|c|c|c|c|}
\hline $\begin{array}{l}\text { Fragment } \\
\text { no. }\end{array}$ & $\begin{array}{l}\text { Colony } \\
\text { no. }\end{array}$ & $\begin{array}{l}\text { Treatment } \\
\text { Temperature }\end{array}$ & $S_{\mathrm{H}}: S_{\mathrm{T}}$ & $\begin{array}{l}\text { Surface } \\
\text { area }\left(\mathrm{cm}^{2}\right)\end{array}$ & $\begin{array}{l}\text { Host protein } \\
\left(\mathrm{mg} \mathrm{cm}^{-2}\right)\end{array}$ & $\begin{array}{l}\text { Symbiont density } \\
\left(\times \mathbf{1 0}^{3} \text { cells } \mathrm{mg}^{-1}\right)\end{array}$ & $\begin{array}{l}\text { Symbiont density } \\
\left(\times 10^{3} \text { cells } \mathrm{cm}^{-2}\right)\end{array}$ & $\begin{array}{l}\text { Chlorophyll } a \\
\left(\text { pg cell }^{-1}\right)\end{array}$ & $\begin{array}{l}\text { Chlorophyll } c_{2} \\
\left(\text { pg cell }^{-1}\right)\end{array}$ & $\begin{array}{l}\text { Chlorophyll } \\
c_{2}: a \text { ratio }\end{array}$ \\
\hline 1 & 1 & $25^{\circ} \mathrm{C}$ & 0.676 & 5.71 & 0.351 & 1314 & 461 & 3.98 & 0.97 & 0.243 \\
\hline 2 & 2 & $25^{\circ} \mathrm{C}$ & 0.704 & 5.29 & 0.754 & 952 & 717 & 2.46 & 1.26 & 0.491 \\
\hline 3 & 3 & $25^{\circ} \mathrm{C}$ & 0.47 & 6.58 & 0.436 & 1036 & 452 & 2.84 & 1.1 & 0.387 \\
\hline 4 & 4 & $25^{\circ} \mathrm{C}$ & 0.565 & 5.96 & 0.57 & 843 & 480 & 2.66 & 1.25 & 0.461 \\
\hline 5 & 5 & $25^{\circ} \mathrm{C}$ & 0.545 & 6.11 & 0.442 & 1052 & 465 & 2.8 & 1.27 & 0.458 \\
\hline 6 & 6 & $25^{\circ} \mathrm{C}$ & 0.637 & 4.38 & 0.489 & 1070 & 524 & 3.03 & 1.15 & 0.406 \\
\hline 7 & 7 & $25^{\circ} \mathrm{C}$ & 0.302 & 3.86 & 0.45 & 1582 & 713 & 3.05 & 0.97 & 0.294 \\
\hline 8 & 8 & $25^{\circ} \mathrm{C}$ & 0.63 & 6.38 & 0.313 & 1127 & 353 & 3.38 & 1.02 & 0.301 \\
\hline 9 & 9 & $25^{\circ} \mathrm{C}$ & 0.497 & 4.32 & 0.405 & 1325 & 537 & 3.03 & 1.1 & 0.362 \\
\hline 10 & 10 & $25^{\circ} \mathrm{C}$ & 0.468 & 5.16 & 0.508 & 1744 & 886 & 3.33 & 1.12 & 0.343 \\
\hline 11 & 11 & $25^{\circ} \mathrm{C}$ & 0.537 & 8.76 & 0.435 & 762 & 332 & 3.67 & 0.89 & 0.242 \\
\hline 12 & 12 & $25^{\circ} \mathrm{C}$ & 0.247 & 5.41 & 0.328 & 2461 & 807 & 2.84 & 0.9 & 0.317 \\
\hline 13 & 13 & $25^{\circ} \mathrm{C}$ & 0.194 & 7.26 & 0.462 & 1056 & 488 & 3.63 & 0.84 & 0.232 \\
\hline 14 & 14 & $25^{\circ} \mathrm{C}$ & 0.277 & 5.44 & 0.342 & 1702 & 582 & 4.17 & 0.71 & 0.171 \\
\hline 15 & 15 & $25^{\circ} \mathrm{C}$ & 0.202 & 4.26 & 0.47 & 1344 & 632 & 4.33 & 1.07 & 0.26 \\
\hline 16 & 1 & $29^{\circ} \mathrm{C}$ & 0.696 & 7.77 & 0.374 & 643 & 240 & 2.08 & 1.06 & 0.509 \\
\hline 17 & 2 & $29^{\circ} \mathrm{C}$ & 0.716 & 3.74 & 0.654 & 635 & 415 & 3.77 & 1.67 & 0.443 \\
\hline 18 & 3 & $29^{\circ} \mathrm{C}$ & 0.448 & 4.57 & 0.422 & 912 & 385 & 3.88 & 1.12 & 0.288 \\
\hline 19 & 4 & $29^{\circ} \mathrm{C}$ & 0.538 & 3.64 & 0.632 & 630 & 398 & 2.91 & 1.44 & 0.494 \\
\hline 20 & 5 & $29^{\circ} \mathrm{C}$ & 0.547 & 4.41 & 0.535 & 802 & 429 & 2.99 & 1.63 & 0.513 \\
\hline 21 & 6 & $29^{\circ} \mathrm{C}$ & 0.616 & 4.75 & 0.438 & 1224 & 536 & 4.96 & 1.66 & 0.345 \\
\hline 22 & 7 & $29^{\circ} \mathrm{C}$ & 0.15 & 5.06 & 0.295 & 1549 & 457 & 3.26 & 0.97 & 0.298 \\
\hline 23 & 8 & $29^{\circ} \mathrm{C}$ & 0.608 & 8.28 & 0.325 & 1118 & 363 & 3.17 & 1.05 & 0.331 \\
\hline 24 & 9 & $29^{\circ} \mathrm{C}$ & 0.563 & 4.55 & 0.488 & 842 & 411 & 3.72 & 1.3 & 0.356 \\
\hline 25 & 10 & $29^{\circ} \mathrm{C}$ & 0.47 & 4.97 & 0.276 & 1961 & 540 & 3.69 & 1.1 & 0.311 \\
\hline 26 & 11 & $29^{\circ} \mathrm{C}$ & 0.562 & 6.11 & 0.424 & 822 & 349 & 2.97 & 0.85 & 0.289 \\
\hline 27 & 12 & $29^{\circ} \mathrm{C}$ & 0.237 & 4.57 & 0.264 & 2081 & 548 & 2.99 & 1.02 & 0.34 \\
\hline 28 & 13 & $29^{\circ} \mathrm{C}$ & 0.255 & 5.43 & 0.471 & 955 & 450 & 3.64 & 1.27 & 0.348 \\
\hline 29 & 14 & $29^{\circ} \mathrm{C}$ & 0.26 & 4.98 & 0.515 & 1224 & 630 & 3.61 & 1.22 & 0.326 \\
\hline 30 & 15 & $29^{\circ} \mathrm{C}$ & 0.223 & 4.71 & 0.432 & 1124 & 486 & 3.86 & 0.92 & 0.255 \\
\hline
\end{tabular}


Table B.17 Photosynthesis-irradiance measurements (chapter 4)

\begin{tabular}{|c|c|c|c|c|c|c|c|c|c|}
\hline $\begin{array}{l}\text { Fragment } \\
\text { no. }\end{array}$ & $\begin{array}{l}\text { Colony } \\
\text { no. }\end{array}$ & $\begin{array}{l}\text { Treatment } \\
\text { temperature }\end{array}$ & $S_{\mathrm{H}}: S_{\mathrm{T}}$ & $\begin{array}{l}P_{G R O S S}(\mu \mathrm{mol} \mathrm{O} \\
\left.\text { mg }^{-1} \text { protein } \mathbf{h}^{-1}\right)\end{array}$ & $\begin{array}{l}R\left(\mu \mathrm{mol} \mathrm{O} \mathrm{Omg}^{-1}\right. \\
\left.\text { protein } \mathrm{h}^{-1}\right)\end{array}$ & $P: R$ & $\begin{array}{l}\alpha\left(\mu \mathrm{mol} \mathrm{O} \mathrm{O}_{2} \mathrm{mg}^{-1} \text { protein } \mathrm{h}^{-1}\right. \\
\left.\left(\mu \mathrm{mol} \text { photons } \mathrm{m}^{-2} \mathrm{~s}^{-1}\right)^{-1}\right)\end{array}$ & $\begin{array}{l}I_{K}(\mu \mathrm{mol} \\
\left.\text { photons } \mathrm{m}^{-2} \mathrm{~s}^{-1}\right)\end{array}$ & $\begin{array}{l}I_{C}(\mu \mathrm{mol} \\
\left.\text { photons } \mathrm{m}^{-2} \mathrm{~s}^{-1}\right)\end{array}$ \\
\hline 1 & 1 & $25^{\circ} \mathrm{C}$ & 0.676 & 1.95 & -1.24 & 1.57 & 0.0203 & 95.8 & 72.4 \\
\hline 2 & 2 & $25^{\circ} \mathrm{C}$ & 0.704 & 1.52 & -0.91 & 1.67 & 0.0172 & 88.5 & 61 \\
\hline 3 & 3 & $25^{\circ} \mathrm{C}$ & 0.47 & 1.09 & -0.61 & 1.77 & 0.0126 & 86.5 & 55.3 \\
\hline 4 & 4 & $25^{\circ} \mathrm{C}$ & 0.565 & 1.35 & -0.85 & 1.59 & 0.0124 & 108.5 & 80.4 \\
\hline 5 & 5 & $25^{\circ} \mathrm{C}$ & 0.545 & 1.53 & -0.88 & 1.74 & 0.0176 & 87.2 & 57.2 \\
\hline 6 & 6 & $25^{\circ} \mathrm{C}$ & 0.637 & 1.25 & -0.65 & 1.93 & 0.0141 & 88.4 & 50.8 \\
\hline 7 & 7 & $25^{\circ} \mathrm{C}$ & 0.302 & 2.52 & -1.36 & 1.86 & 0.0239 & 105.6 & 63.5 \\
\hline 8 & 8 & $25^{\circ} \mathrm{C}$ & 0.63 & 1.9 & -1.16 & 1.64 & 0.0234 & 80.9 & 57.4 \\
\hline 9 & 9 & $25^{\circ} \mathrm{C}$ & 0.497 & 1.68 & -1.09 & 1.54 & 0.0198 & 84.9 & 65.5 \\
\hline 10 & 10 & $25^{\circ} \mathrm{C}$ & 0.468 & 1.29 & -0.72 & 1.79 & 0.0135 & 95.6 & 60.2 \\
\hline 11 & 11 & $25^{\circ} \mathrm{C}$ & 0.537 & 1.4 & -1.04 & 1.35 & 0.0154 & 91.1 & 86.4 \\
\hline 12 & 12 & $25^{\circ} \mathrm{C}$ & 0.247 & 2.68 & -1.34 & 2 & 0.0399 & 67.1 & 36.8 \\
\hline 13 & 13 & $25^{\circ} \mathrm{C}$ & 0.194 & 1.59 & -0.79 & 2.02 & 0.0165 & 95.9 & 52 \\
\hline 14 & 14 & $25^{\circ} \mathrm{C}$ & 0.277 & 1.9 & -1.08 & 1.76 & 0.0305 & 62.2 & 40.2 \\
\hline 15 & 15 & $25^{\circ} \mathrm{C}$ & 0.202 & 2.23 & -1.21 & 1.84 & 0.0273 & 81.6 & 49.6 \\
\hline 16 & 1 & $29^{\circ} \mathrm{C}$ & 0.696 & 1.1 & -0.92 & 1.2 & 0.0151 & 73 & 88.2 \\
\hline 17 & 2 & $29^{\circ} \mathrm{C}$ & 0.716 & 1.18 & -0.85 & 1.4 & 0.0084 & 140.9 & 126.7 \\
\hline 18 & 3 & $29^{\circ} \mathrm{C}$ & 0.448 & 2.32 & -1.66 & 1.4 & 0.0347 & 66.9 & 59.8 \\
\hline 19 & 4 & $29^{\circ} \mathrm{C}$ & 0.538 & 1.1 & -1.03 & 1.07 & 0.0114 & 96.1 & 163.6 \\
\hline 20 & 5 & $29^{\circ} \mathrm{C}$ & 0.547 & 1.54 & -1.2 & 1.28 & 0.0199 & 77.6 & 81.2 \\
\hline 21 & 6 & $29^{\circ} \mathrm{C}$ & 0.616 & 1.7 & -1.09 & 1.56 & 0.0222 & 76.6 & 58.1 \\
\hline 22 & 7 & $29^{\circ} \mathrm{C}$ & 0.15 & 2.98 & -1.92 & 1.55 & 0.0383 & 77.7 & 59.7 \\
\hline 23 & 8 & $29^{\circ} \mathrm{C}$ & 0.608 & 1.93 & -1.32 & 1.46 & 0.0241 & 80 & 67.2 \\
\hline 24 & 9 & $29^{\circ} \mathrm{C}$ & 0.563 & 1.28 & -1.15 & 1.12 & 0.0112 & 114 & 164.9 \\
\hline 25 & 10 & $29^{\circ} \mathrm{C}$ & 0.47 & 2.52 & -1.76 & 1.43 & 0.0216 & 116.7 & 100.6 \\
\hline 26 & 11 & $29^{\circ} \mathrm{C}$ & 0.562 & 1.24 & -1.01 & 1.23 & 0.01 & 124.1 & 141.5 \\
\hline 27 & 12 & $29^{\circ} \mathrm{C}$ & 0.237 & 2.52 & -1.68 & 1.5 & 0.0426 & 59.1 & 47.6 \\
\hline 28 & 13 & $29^{\circ} \mathrm{C}$ & 0.255 & 1.48 & -0.97 & 1.53 & 0.0207 & 71.6 & 56.2 \\
\hline 29 & 14 & $29^{\circ} \mathrm{C}$ & 0.26 & 1.6 & -1.04 & 1.54 & 0.0181 & 88.4 & 68.4 \\
\hline 30 & 15 & $29^{\circ} \mathrm{C}$ & 0.223 & 1.37 & -1.03 & 1.33 & 0.0168 & 81.3 & 79.7 \\
\hline
\end{tabular}


Table B.18 Mortality, growth, and temporal changes in $S_{\mathrm{H}}: S_{\mathrm{T}}$ (chapter 5 )

\begin{tabular}{|c|c|c|c|c|c|c|c|c|c|c|c|c|c|c|c|c|}
\hline Colony & $\begin{array}{l}\begin{array}{l}\text { Original } \\
\text { site }\end{array} \\
\end{array}$ & $\begin{array}{l}\text { Transplant } \\
\text { site } \\
\end{array}$ & $\begin{array}{l}\text { Survived } \\
195 \mathrm{~d}\end{array}$ & $\begin{array}{l}\text { Survived } \\
\text { 374 d } \\
\end{array}$ & $\begin{array}{l}\text { Initial dry } \\
\text { weight (g) }\end{array}$ & $\begin{array}{l}\text { Final dry } \\
\text { weight (g) }\end{array}$ & $\begin{array}{l}\text { Time } \\
\text { (d) }\end{array}$ & $\begin{array}{l}\text { Growth } \\
\left(\text { mg d }^{-1}\right)\end{array}$ & $\begin{array}{l}\text { Growth } \\
\left(\times 10^{3} \% d^{-1}\right)\end{array}$ & $\begin{array}{l}\text { Original } \\
S_{\mathrm{H}}: S_{\mathrm{T}} \\
\end{array}$ & $\begin{array}{l}\text { Mean C } \mathrm{C}_{\mathrm{t}} \\
\left(\mathrm{C100}^{+}\right)\end{array}$ & $\begin{array}{l}\begin{array}{l}\text { Mean C } \\
\left(\mathrm{C100}^{-}\right)\end{array} \\
\end{array}$ & $\mathbf{C}_{\mathrm{C} 100}$ & $\mathbf{C}_{\text {TOtAL }}$ & $\begin{array}{l}\mathbf{C}_{100}: \\
\mathbf{C}_{\text {TOTAL }} \\
\end{array}$ & $\begin{array}{l}\text { Final } \\
S_{\mathrm{H}}: S_{\mathrm{T}} \\
\end{array}$ \\
\hline \multirow[t]{6}{*}{1} & North Bay & North Bay & $\mathrm{Y}$ & $* \mathrm{~N} / \mathrm{A}$ & 6.20 & 8.91 & 195 & 13.88 & 1.86 & 0.70 & 23.41 & 24.79 & 9655 & 14175 & 0.68 & 0.69 \\
\hline & & North Bay & Y & $\mathrm{Y}$ & 9.05 & 26.26 & 374 & 46.02 & 2.85 & & 24.61 & 25.64 & 4251 & 6783 & 0.63 & 0.75 \\
\hline & & North Bay & Y & Y & 6.30 & 14.28 & 374 & 21.33 & 2.19 & & 24.70 & 25.86 & 4001 & 6184 & 0.65 & 0.73 \\
\hline & & The Arch & Y & $* \mathrm{~N} / \mathrm{A}$ & 5.80 & 8.97 & 195 & 16.26 & 2.24 & & 25.14 & 26.22 & 2962 & 4669 & 0.63 & 0.74 \\
\hline & & The Arch & Y & $\mathrm{N}$ & 8.26 & & & & & & & & & & & \\
\hline & & The Arch & Y & $\mathrm{N}$ & 7.52 & & & & & & & & & & & \\
\hline \multirow[t]{6}{*}{2} & North Bay & North Bay & Y & $* \mathrm{~N} / \mathrm{A}$ & 10.37 & 13.66 & 195 & 16.87 & 1.41 & 0.65 & 21.90 & 23.55 & 26925 & 37475 & 0.72 & 0.63 \\
\hline & & North Bay & Y & $\mathrm{Y}$ & 10.50 & 24.76 & 374 & 38.12 & 2.30 & & 23.65 & 25.20 & 8188 & 11613 & 0.71 & 0.65 \\
\hline & & North Bay & Y & $\mathrm{Y}$ & 7.99 & 20.22 & 374 & 32.69 & 2.49 & & 24.27 & 25.90 & 5372 & 7488 & 0.72 & 0.64 \\
\hline & & The Arch & Y & $* \mathrm{~N} / \mathrm{A}$ & 10.58 & 15.78 & 195 & 26.69 & 2.05 & & 28.78 & 30.38 & 248 & 348 & 0.71 & 0.64 \\
\hline & & The Arch & $\mathrm{N}$ & $\mathrm{N}$ & 9.50 & & & & & & & & & & & \\
\hline & & The Arch & Y & $\mathrm{N}$ & 7.25 & & & & & & & & & & & \\
\hline \multirow[t]{6}{*}{3} & North Bay & North Bay & Y & $* \mathrm{~N} / \mathrm{A}$ & 9.73 & 13.03 & 195 & 16.91 & 1.50 & 0.60 & 23.97 & 26.13 & 6600 & 8409 & 0.78 & 0.51 \\
\hline & & North Bay & Y & $\mathrm{Y}$ & 12.36 & 21.34 & 374 & 24.02 & 1.46 & & 28.37 & 30.22 & 328 & 439 & 0.75 & 0.59 \\
\hline & & North Bay & Y & $\mathrm{Y}$ & 4.80 & 10.71 & 374 & 15.80 & 2.15 & & 24.64 & 26.70 & 4183 & 5408 & 0.77 & 0.54 \\
\hline & & The Arch & Y & $* \mathrm{~N} / \mathrm{A}$ & 4.66 & 6.11 & 195 & 7.44 & 1.39 & & 30.71 & 33.01 & 67 & 84 & 0.80 & 0.48 \\
\hline & & The Arch & Y & $\mathrm{N}$ & 17.14 & & & & & & & & & & & \\
\hline & & The Arch & Y & $\mathrm{Y}$ & 8.25 & 7.55 & 374 & -1.86 & -0.24 & & 23.16 & 24.94 & 11413 & 15504 & 0.74 & 0.61 \\
\hline \multirow[t]{6}{*}{4} & North Bay & North Bay & Y & $* \mathrm{~N} / \mathrm{A}$ & 11.66 & 15.36 & 195 & 18.96 & 1.41 & 0.62 & 25.62 & 27.12 & 2139 & 3063 & 0.70 & 0.67 \\
\hline & & North Bay & Y & $\mathrm{Y}$ & 10.95 & 31.49 & 374 & 54.92 & 2.83 & & 23.41 & 24.98 & 9657 & 13627 & 0.71 & 0.65 \\
\hline & & North Bay & Y & $\mathrm{Y}$ & 10.70 & 14.72 & 374 & 10.74 & 0.85 & & 24.82 & 26.52 & 3694 & 5086 & 0.73 & 0.62 \\
\hline & & The Arch & Y & $* \mathrm{~N} / \mathrm{A}$ & 11.39 & 14.02 & 195 & 13.47 & 1.06 & & 25.43 & 27.07 & 2437 & 3387 & 0.72 & 0.63 \\
\hline & & The Arch & Y & $\mathrm{N}$ & 12.16 & & & & & & & & & & & \\
\hline & & The Arch & Y & $\mathrm{N}$ & 10.18 & & & & & & & & & & & \\
\hline \multirow[t]{6}{*}{5} & North Bay & North Bay & Y & $* \mathrm{~N} / \mathrm{A}$ & 10.95 & 15.29 & 195 & 22.27 & 1.71 & 0.50 & 25.04 & 27.55 & 3177 & 3865 & 0.82 & 0.43 \\
\hline & & North Bay & Y & $\mathrm{Y}$ & 13.48 & 19.31 & 374 & 15.59 & 0.96 & & 23.05 & 25.21 & 12286 & 15676 & 0.78 & 0.52 \\
\hline & & North Bay & Y & $\mathrm{Y}$ & 14.13 & 26.34 & 374 & 32.65 & 1.67 & & 25.33 & 27.54 & 2607 & 3299 & 0.79 & 0.50 \\
\hline & & The Arch & Y & $* \mathrm{~N} / \mathrm{A}$ & 12.92 & 16.37 & 195 & 17.71 & 1.22 & & 26.59 & 28.76 & 1103 & 1404 & 0.79 & 0.51 \\
\hline & & The Arch & Y & $\mathrm{N}$ & 13.47 & & & & & & & & & & & \\
\hline & & The Arch & Y & $\mathrm{Y}$ & 9.78 & 8.54 & 374 & -3.32 & -0.36 & & 28.88 & 30.90 & 232 & 302 & 0.77 & 0.55 \\
\hline \multirow[t]{2}{*}{6} & North Bay & North Bay & Y & $* \mathrm{~N} / \mathrm{A}$ & 5.80 & 10.81 & 195 & 25.69 & 3.20 & 0.59 & 27.35 & 29.32 & 657 & 862 & 0.76 & 0.56 \\
\hline & & North Bay & Y & $\mathrm{Y}$ & 7.99 & 13.66 & 374 & 15.17 & 1.44 & & 25.30 & 26.83 & 2655 & 3775 & 0.70 & 0.66 \\
\hline
\end{tabular}




\begin{tabular}{|c|c|c|c|c|c|c|c|c|c|c|c|c|c|c|c|c|}
\hline & & North Bay & $\mathrm{Y}$ & $\mathrm{Y}$ & 9.75 & 20.51 & 374 & 28.76 & 1.99 & & 22.96 & 24.75 & 13078 & 17730 & 0.74 & 0.60 \\
\hline & & The Arch & $\mathrm{Y}$ & $* \mathrm{~N} / \mathrm{A}$ & 6.41 & 11.93 & 195 & 28.31 & 3.19 & & 24.78 & 26.42 & 3782 & 5269 & 0.72 & 0.64 \\
\hline & & The Arch & $\mathrm{N}$ & $\mathrm{N}$ & 3.91 & & & & & & & & & & & \\
\hline & & The Arch & $\mathrm{Y}$ & $\mathrm{Y}$ & 6.41 & 11.27 & 374 & 12.99 & 1.51 & & 23.59 & 25.33 & 8526 & 11658 & 0.73 & 0.61 \\
\hline \multirow[t]{6}{*}{7} & North Bay & North Bay & $\mathrm{Y}$ & $* \mathrm{~N} / \mathrm{A}$ & 11.81 & 14.63 & 195 & 14.48 & 1.10 & 0.22 & 22.23 & 24.61 & 21575 & 26676 & 0.81 & 0.46 \\
\hline & & North Bay & $\mathrm{Y}$ & $\mathrm{Y}$ & 6.75 & 12.97 & 374 & 16.63 & 1.75 & & 21.66 & 25.63 & 31702 & 34251 & 0.93 & 0.16 \\
\hline & & North Bay & $\mathrm{Y}$ & $\mathrm{Y}$ & 7.70 & 16.92 & 374 & 24.66 & 2.11 & & 22.37 & 25.43 & 19655 & 22573 & 0.87 & 0.31 \\
\hline & & The Arch & $\mathrm{Y}$ & $* \mathrm{~N} / \mathrm{A}$ & 9.12 & 9.80 & 195 & 3.48 & 0.37 & & 22.30 & 25.80 & 20530 & 22794 & 0.90 & 0.23 \\
\hline & & The Arch & $\mathrm{Y}$ & $\mathrm{N}$ & 5.72 & & & & & & & & & & & \\
\hline & & The Arch & $\mathrm{Y}$ & $\mathrm{N}$ & 6.57 & & & & & & & & & & & \\
\hline \multirow[t]{6}{*}{8} & North Bay & North Bay & $\mathrm{Y}$ & $*$ N/A & 7.12 & 10.87 & 195 & 19.24 & 2.17 & 0.23 & 23.03 & 26.28 & 12506 & 14144 & 0.88 & 0.28 \\
\hline & & North Bay & $\mathrm{Y}$ & $\mathrm{Y}$ & 12.23 & 26.87 & 374 & 39.15 & 2.11 & & 24.36 & 27.59 & 5057 & 5725 & 0.88 & 0.28 \\
\hline & & North Bay & $\mathrm{Y}$ & $\mathrm{Y}$ & 5.46 & 10.92 & 374 & 14.60 & 1.86 & & 25.88 & 29.05 & 1787 & 2033 & 0.88 & 0.29 \\
\hline & & The Arch & $\mathrm{Y}$ & $* \mathrm{~N} / \mathrm{A}$ & 14.06 & 22.09 & 195 & 41.20 & 2.32 & & 24.68 & 28.04 & 4071 & 4562 & 0.89 & 0.25 \\
\hline & & The Arch & $\mathrm{N}$ & $\mathrm{N}$ & 4.54 & & & & & & & & & & & \\
\hline & & The Arch & $\mathrm{Y}$ & $\mathrm{Y}$ & 7.73 & 9.25 & 374 & 4.06 & 0.48 & & 22.58 & 26.14 & 16923 & 18725 & 0.90 & 0.22 \\
\hline \multirow[t]{6}{*}{9} & North Bay & North Bay & $\mathrm{Y}$ & $* \mathrm{~N} / \mathrm{A}$ & 3.62 & 6.45 & 195 & 14.52 & 2.97 & 0.65 & 25.70 & 27.20 & 2030 & 2902 & 0.70 & 0.66 \\
\hline & & North Bay & $\mathrm{Y}$ & $\mathrm{N}$ & 5.56 & & & & & & & & & & & \\
\hline & & North Bay & $\mathrm{Y}$ & $\mathrm{Y}$ & 8.25 & 15.42 & 374 & 19.17 & 1.67 & & 23.68 & 25.24 & 8019 & 11349 & 0.71 & 0.65 \\
\hline & & The Arch & $\mathrm{Y}$ & $* \mathrm{~N} / \mathrm{A}$ & 3.13 & 4.55 & 195 & 7.27 & 1.92 & & 23.49 & 25.03 & 9126 & 12970 & 0.70 & 0.66 \\
\hline & & The Arch & $\mathrm{N}$ & $\mathrm{N}$ & 3.56 & & & & & & & & & & & \\
\hline & & The Arch & $\mathrm{Y}$ & $\mathrm{N}$ & 7.75 & & & & & & & & & & & \\
\hline \multirow[t]{6}{*}{10} & North Bay & North Bay & $\mathrm{Y}$ & $*$ N/A & 7.30 & 9.37 & 195 & 10.63 & 1.28 & 0.75 & 24.02 & 25.33 & 6356 & 9480 & 0.67 & 0.70 \\
\hline & & North Bay & $\mathrm{Y}$ & $\mathrm{Y}$ & 5.01 & 8.10 & 374 & 8.27 & 1.29 & & 26.66 & 27.92 & 1056 & 1589 & 0.66 & 0.71 \\
\hline & & North Bay & $\mathrm{Y}$ & $\mathrm{Y}$ & 7.14 & 15.86 & 374 & 23.32 & 2.14 & & 25.61 & 26.80 & 2157 & 3302 & 0.65 & 0.72 \\
\hline & & The Arch & $\mathrm{Y}$ & $* \mathrm{~N} / \mathrm{A}$ & 8.13 & 10.29 & 195 & 11.08 & 1.21 & & 23.98 & 25.18 & 6549 & 10014 & 0.65 & 0.72 \\
\hline & & The Arch & $\mathrm{Y}$ & $\mathrm{N}$ & 6.07 & & & & & & & & & & & \\
\hline & & The Arch & $\mathrm{Y}$ & $\mathrm{N}$ & 10.63 & & & & & & & & & & & \\
\hline \multirow[t]{6}{*}{11} & The Arch & North Bay & $\mathrm{Y}$ & $*$ N/A & 2.87 & 4.22 & 195 & 6.94 & 1.98 & 0.36 & 24.86 & 27.95 & 3581 & 4105 & 0.87 & 0.31 \\
\hline & & North Bay & $\mathrm{Y}$ & $\mathrm{Y}$ & 4.38 & 7.91 & 374 & 9.44 & 1.58 & & 26.15 & 28.77 & 1489 & 1787 & 0.83 & 0.41 \\
\hline & & North Bay & $\mathrm{Y}$ & $\mathrm{Y}$ & 3.91 & 7.07 & 374 & 8.45 & 1.59 & & 24.39 & 27.21 & 4958 & 5824 & 0.85 & 0.36 \\
\hline & & The Arch & $\mathrm{Y}$ & $* \mathrm{~N} / \mathrm{A}$ & 3.29 & 4.84 & 195 & 7.94 & 1.98 & & 27.37 & 30.75 & 651 & 729 & 0.89 & 0.25 \\
\hline & & The Arch & $\mathrm{Y}$ & $\mathrm{N}$ & 4.80 & & & & & & & & & & & \\
\hline & & The Arch & $\mathrm{Y}$ & $\mathrm{N}$ & 4.22 & & & & & & & & & & & \\
\hline 12 & The Arch & North Bay & $\mathrm{Y}$ & $*$ N/A & 2.53 & 3.51 & 195 & 5.02 & 1.68 & 0.57 & 22.06 & 24.06 & 24190 & 31640 & 0.76 & 0.55 \\
\hline
\end{tabular}




\begin{tabular}{|c|c|c|c|c|c|c|c|c|c|c|c|c|c|c|c|c|}
\hline & & North Bay & $\mathrm{Y}$ & $\mathrm{Y}$ & 8.09 & 15.57 & 374 & 20.00 & 1.75 & & 24.46 & 26.43 & 4719 & 6198 & 0.76 & 0.56 \\
\hline & & North Bay & $\mathrm{Y}$ & $\mathrm{Y}$ & 3.21 & 5.05 & 374 & 4.92 & 1.21 & & 25.37 & 27.18 & 2531 & 3417 & 0.74 & 0.60 \\
\hline & & The Arch & $\mathrm{Y}$ & $* \mathrm{~N} / \mathrm{A}$ & 5.49 & 8.58 & 195 & 15.87 & 2.29 & & 26.49 & 28.00 & 1182 & 1687 & 0.70 & 0.66 \\
\hline & & The Arch & $\mathrm{N}$ & $\mathrm{N}$ & 1.64 & & & & & & & & & & & \\
\hline & & The Arch & $\mathrm{Y}$ & $\mathrm{N}$ & 6.28 & & & & & & & & & & & \\
\hline \multirow[t]{6}{*}{13} & The Arch & North Bay & $\mathrm{Y}$ & $* \mathrm{~N} / \mathrm{A}$ & 4.43 & 5.70 & 195 & 6.53 & 1.30 & 0.69 & 26.03 & 27.74 & 1619 & 2223 & 0.73 & 0.62 \\
\hline & & North Bay & $\mathrm{Y}$ & $\mathrm{N}$ & 3.03 & & & & & & & & & & & \\
\hline & & North Bay & $\mathrm{Y}$ & Y & 2.08 & 3.97 & 374 & 5.05 & 1.73 & & 25.14 & 26.34 & 2968 & 4538 & 0.65 & 0.72 \\
\hline & & The Arch & $\mathrm{Y}$ & $* \mathrm{~N} / \mathrm{A}$ & 2.77 & 4.82 & 195 & 10.52 & 2.85 & & 25.33 & 26.85 & 2610 & 3717 & 0.70 & 0.66 \\
\hline & & The Arch & $\mathrm{N}$ & $\mathrm{N}$ & 4.25 & & & & & & & & & & & \\
\hline & & The Arch & $\mathrm{Y}$ & $\mathrm{Y}$ & 3.80 & 8.90 & 374 & 13.64 & 2.28 & & 24.88 & 26.33 & 3539 & 5115 & 0.69 & 0.67 \\
\hline \multirow[t]{6}{*}{14} & The Arch & North Bay & $\mathrm{Y}$ & $*$ N/A & 11.36 & 15.01 & 195 & 18.73 & 1.43 & 0.59 & 25.29 & 27.78 & 2676 & 3263 & 0.82 & 0.44 \\
\hline & & North Bay & $\mathrm{N}$ & $\mathrm{N}$ & 7.97 & & & & & & & & & & & \\
\hline & & North Bay & $\mathrm{Y}$ & $\mathrm{Y}$ & 13.14 & 27.10 & 374 & 37.33 & 1.94 & & 23.50 & 25.47 & 9086 & 11922 & 0.76 & 0.56 \\
\hline & & The Arch & $\mathrm{Y}$ & $* \mathrm{~N} / \mathrm{A}$ & 6.62 & 6.45 & 195 & -0.86 & -0.13 & & 25.21 & 27.39 & 2833 & 3601 & 0.79 & 0.51 \\
\hline & & The Arch & $\mathrm{Y}$ & $\mathrm{N}$ & 10.04 & & & & & & & & & & & \\
\hline & & The Arch & $\mathrm{Y}$ & $\mathrm{N}$ & 4.72 & & & & & & & & & & & \\
\hline \multirow[t]{6}{*}{15} & The Arch & North Bay & $\mathrm{Y}$ & $*$ N/A & 5.62 & 7.07 & 195 & 7.41 & 1.17 & 0.73 & 25.65 & 26.86 & 2103 & 3200 & 0.66 & 0.72 \\
\hline & & North Bay & $\mathrm{Y}$ & $\mathrm{Y}$ & 3.43 & 4.04 & 374 & 1.64 & 0.44 & & 26.52 & 27.86 & 1158 & 1715 & 0.68 & 0.70 \\
\hline & & North Bay & $\mathrm{Y}$ & Y & 3.37 & 4.65 & 374 & 3.42 & 0.86 & & 24.93 & 26.37 & 3422 & 4954 & 0.69 & 0.68 \\
\hline & & The Arch & $\mathrm{Y}$ & $* \mathrm{~N} / \mathrm{A}$ & 9.57 & 10.70 & 195 & 5.78 & 0.57 & & 25.25 & 26.81 & 2749 & 3885 & 0.71 & 0.65 \\
\hline & & The Arch & $\mathrm{N}$ & $\mathrm{N}$ & 3.59 & & & & & & & & & & & \\
\hline & & The Arch & $\mathrm{N}$ & $\mathrm{N}$ & 3.01 & & & & & & & & & & & \\
\hline \multirow[t]{6}{*}{16} & The Arch & North Bay & $\mathrm{Y}$ & *N/A & 5.73 & 7.90 & 195 & 11.13 & 1.65 & 0.58 & 25.23 & 26.76 & 2782 & 3961 & 0.70 & 0.66 \\
\hline & & North Bay & $\mathrm{Y}$ & $\mathrm{Y}$ & 10.60 & 20.74 & 374 & 27.11 & 1.80 & & 22.29 & 24.10 & 20638 & 27895 & 0.74 & 0.60 \\
\hline & & North Bay & $\mathrm{Y}$ & $\mathrm{Y}$ & 6.38 & 13.52 & 374 & 19.08 & 2.01 & & 25.21 & 26.68 & 2830 & 4078 & 0.69 & 0.67 \\
\hline & & The Arch & $\mathrm{Y}$ & $* \mathrm{~N} / \mathrm{A}$ & 3.99 & 6.02 & 195 & 10.43 & 2.11 & & 24.26 & 26.04 & 5407 & 7334 & 0.74 & 0.60 \\
\hline & & The Arch & $\mathrm{Y}$ & $\mathrm{N}$ & 11.74 & & & & & & & & & & & \\
\hline & & The Arch & $\mathrm{Y}$ & $\mathrm{Y}$ & 6.14 & 11.24 & 374 & 13.62 & 1.62 & & 24.91 & 26.59 & 3464 & 4785 & 0.72 & 0.63 \\
\hline \multirow[t]{6}{*}{17} & The Arch & North Bay & $\mathrm{Y}$ & *N/A & 3.61 & 5.00 & 195 & 7.14 & 1.67 & 0.64 & 26.18 & 27.72 & 1462 & 2072 & 0.71 & 0.65 \\
\hline & & North Bay & $\mathrm{Y}$ & $\mathrm{Y}$ & 8.65 & 38.83 & 374 & 80.69 & 4.02 & & 23.27 & 25.09 & 10641 & 14321 & 0.74 & 0.59 \\
\hline & & North Bay & $\mathrm{Y}$ & Y & 5.17 & 20.48 & 374 & 40.92 & 3.69 & & 23.79 & 25.73 & 7439 & 9820 & 0.76 & 0.57 \\
\hline & & The Arch & $\mathrm{Y}$ & $* \mathrm{~N} / \mathrm{A}$ & 3.33 & 6.15 & 195 & 14.45 & 3.15 & & 24.31 & 26.36 & 5224 & 6771 & 0.77 & 0.54 \\
\hline & & The Arch & $\mathrm{N}$ & $\mathrm{N}$ & 5.38 & & & & & & & & & & & \\
\hline & & The Arch & $\mathrm{Y}$ & $\mathrm{N}$ & 9.70 & & & & & & & & & & & \\
\hline
\end{tabular}




\begin{tabular}{|c|c|c|c|c|c|c|c|c|c|c|c|c|c|c|c|c|}
\hline \multirow[t]{6}{*}{18} & The Arch & North Bay & Y & $*$ N/A & 3.96 & 5.37 & 195 & 7.22 & 1.56 & 0.70 & 26.77 & 28.56 & 976 & 1322 & 0.74 & 0.60 \\
\hline & & North Bay & $\mathrm{Y}$ & Y & 9.57 & 22.45 & 374 & 34.44 & 2.28 & & 25.52 & 27.38 & 2290 & 3063 & 0.75 & 0.59 \\
\hline & & North Bay & Y & Y & 2.27 & 5.01 & 374 & 7.34 & 2.12 & & 25.57 & 27.55 & 2214 & 2900 & 0.76 & 0.56 \\
\hline & & The Arch & $\mathrm{Y}$ & $* \mathrm{~N} / \mathrm{A}$ & 4.64 & 6.92 & 195 & 11.70 & 2.05 & & 24.66 & 26.53 & 4112 & 5495 & 0.75 & 0.58 \\
\hline & & The Arch & $\mathrm{N}$ & $\mathrm{N}$ & 2.92 & & & & & & & & & & & \\
\hline & & The Arch & $\mathrm{Y}$ & $\mathrm{N}$ & 7.30 & & & & & & & & & & & \\
\hline \multirow[t]{6}{*}{19} & The Arch & North Bay & $\mathrm{Y}$ & $*$ N/A & 7.81 & 10.49 & 195 & 13.73 & 1.51 & 0.70 & 24.86 & 26.29 & 3600 & 5227 & 0.69 & 0.68 \\
\hline & & North Bay & $\mathrm{Y}$ & $\mathrm{N}$ & 2.35 & & & & & & & & & & & \\
\hline & & North Bay & $\mathrm{Y}$ & Y & 4.72 & 8.35 & 374 & 9.72 & 1.53 & & 22.95 & 24.54 & 13217 & 18585 & 0.71 & 0.65 \\
\hline & & The Arch & $\mathrm{Y}$ & $* \mathrm{~N} / \mathrm{A}$ & 7.23 & 13.13 & 195 & 30.28 & 3.07 & & 23.32 & 24.83 & 10268 & 14683 & 0.70 & 0.66 \\
\hline & & The Arch & $\mathrm{Y}$ & $\mathrm{N}$ & 10.31 & & & & & & & & & & & \\
\hline & & The Arch & $\mathrm{Y}$ & $\mathrm{Y}$ & 3.99 & 5.24 & 374 & 3.34 & 0.73 & & 25.67 & 27.05 & 2068 & 3036 & 0.68 & 0.69 \\
\hline \multirow[t]{6}{*}{20} & The Arch & North Bay & $\mathrm{Y}$ & $* \mathrm{~N} / \mathrm{A}$ & 5.48 & 7.31 & 195 & 9.37 & 1.48 & 0.30 & 24.29 & 27.46 & 5287 & 6017 & 0.88 & 0.29 \\
\hline & & North Bay & $\mathrm{Y}$ & $\mathrm{N}$ & 8.89 & & & & & & & & & & & \\
\hline & & North Bay & $\mathrm{Y}$ & Y & 3.46 & 7.15 & 374 & 9.88 & 1.94 & & 23.16 & 26.16 & 11403 & 13176 & 0.87 & 0.33 \\
\hline & & The Arch & $\mathrm{Y}$ & $* \mathrm{~N} / \mathrm{A}$ & 2.08 & 2.63 & 195 & 2.84 & 1.21 & & 24.05 & 26.98 & 6245 & 7258 & 0.86 & 0.34 \\
\hline & & The Arch & $\mathrm{N}$ & $\mathrm{N}$ & 13.32 & & & & & & & & & & & \\
\hline & & The Arch & $\mathrm{Y}$ & $\mathrm{N}$ & 3.87 & & & & & & & & & & & \\
\hline
\end{tabular}

Asterisks indicate coral fragments collected after $195 \mathrm{~d}$ 
